\section{QUEBEC CITY, QUEBEC JUNE 17-21, 2003}

\section{CCNS Executive Committee}

Peter Bailey, CNS

Qais Ghanem, CSCN

Andrew Kirk, CNS

Daune L. MacGregor, CACN

Brian Wheelock, CNSS

Robert G. Lee, Executive VP

\section{Local Arrangements Contacts}

Jean-Pierre Bouchard

Richard Desbiens

\section{Scientific Program Committee}

Chair: J. Max Findlay

Timothy Benstead

Gregory Cairncross

Sultan Darvesh

Richard Desbiens

Mark Freedman

Christopher Honey

Mano Javidan

Ivar Mendez

Michael Shevell

Christopher Wallace

Shelly Weiss

Canadian Congress of Neurological Sciences

709 - 7015 Macleod Trail SW,

Calgary, AB Canada T2H 2K6

Tel: (403) 229-9544 • Fax: (403) 229-1661

E-mail: brains@ccns.org 38th MEETING OF THE CANADIAN CONGRESS OF NEUROLOGICAL SCIENCES
The Canadian Journal of Neurological Sciences does not assume any responsibility or liability for any errors in the abstracts.

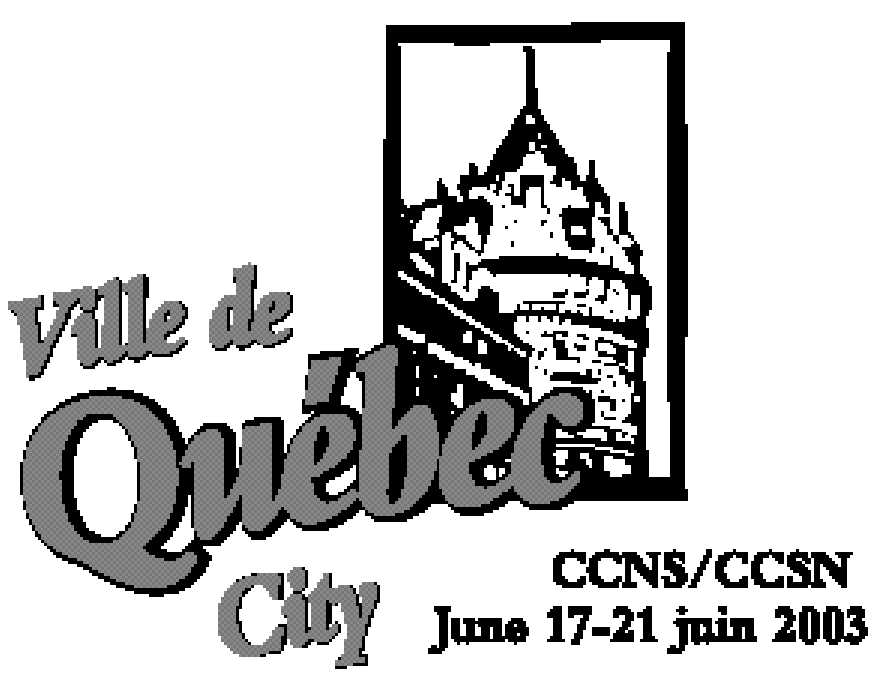




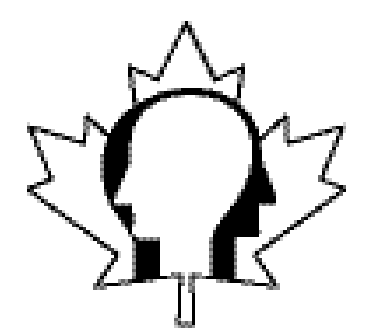

THE CANADIAN JOURNAL OF Neurological Sciences

LE JOURNAL CANADIEN DES
Editor-in-Chief/Rédacteur en chef Douglas W. Zochodne CALGARY, AB

Associate Editors/Rédacteurs associés

William A. Fletcher CALGARY, AB

Andres M. Lozano TORONTO, ON

Past Editors/Anciens rédacteurs en chef

James A. Sharpe TORONTO, ON

Robert G. Lee CALGARY, AB

Robert T. Ross WINNIPEG, MB

(Emeritus Editor, Founding Editor)

Editorial Board/Conseil Scientifique

Timothy J. Benstead HALIFAX, NS

J. Gregory Cairncross CALGARY, AB

Richard Desbiens QUEBEC CITY, QC

J. Max Findlay EDMONTON, AB

Hans-Peter Hartung DUSSELDORF, GERMANY

Renn Holness HALIFAX, NS

Alan C. Jackson KINGSTON, ON

Jack Jhamandas EDMONTON, AB

Douglas Kondziolka PITTSBURGH, PA, USA

Terence Myles CALGARY, AB

John H. Noseworthy ROCHESTER, MN, USA

David Ramsay LONDON, ON

Peter M. Richardson LONDON, UK

Guy Rouleau MONTREAL, QC

Shashi S. Seshia WINNIPEG, MB

Michael Shevell MONTREAL, QC

Paul Steinbok VANCOUVER, BC

Jonathan A. Stoessl VANCOUVER, BC

Samuel Wiebe LONDON, ON

\section{SECTION EDITORS/CONSEIL DE RÉDACTION}

Neuroimaging Highlight/Neuroimagerie

Mark Hudon CALGARY, AB

William Hu CALGARY, AB

Neuropathological Conference/Conférence sur la neuropathologie

David Ramsay LONDON, ON

Book Review/Critiques de livres

Christopher White CALGARY, AB

Managing Director/Gérant directrice

Sally A. Gregg CALGARY, AB

Publications Committee/Comité de Rédaction

G. Bryan Young LONDON, ON

Owen Williams WINNIPG, MB

Joseph Chu ETOBICOKE, ON

Noel Lowry SASKATOON, SK
The official journal of: / La Revue officielle de:

The Canadian Neurological Society

La Société Canadienne de Neurologie

The Canadian Neurosurgical Society

La Société Canadienne de Neurochirurgie

The Canadian Society of Clinical Neurophysiologists

La Société Canadienne de Neurophysiologie Clinique

The Canadian Association of Child Neurology

L'Association Canadienne de Neurologie Pédiatrique

The permanent secretariat for the four societies and the Canadian Congress of Neurological Sciences is at:

Le secrétariat des quatre associations et du Congrès Canadien des Sciences Neurologiques est situe en permanence à:

7015 Macleod Trail SW, Suite 709, Calgary AB, Canada T2H 2K6,

The Canadian Journal of Neurological Sciences is published quarterly. The annual subscription rate for Individuals are: $\mathrm{C} \$ 80$ (Canada), US\$80 (USA), and US $\$ 85$ (elsewhere). Subscription rates for Institutions are: C $\$ 90$ (Canada), US\$90 (USA), and US\$95 (elsewhere). Resident, intern and student rates are available. See and US\$95 (elsewhere). Resident, intern and student rates are available. See
www.cjns.org for details. Single copies C $\$ 22$ each plus postage and handling. All manuscripts and communications should be sent to: Canadian Journal of Neurological Sciences, P.O. Box 5456, Station A, Calgary, AB Canada T2H 1X8. Courier to: 709 7015 Macleod Trail SW, Calgary, AB Canada T2H 2K6. Telephone (403) 229-9575; Fax (403) 229-1661.E-mail: journal@ @jns.org; Website: www.cjns.org COPYRIGHT@ 2003 by THE CANADIAN JOURNALOF NEUROLOGICAL SCIENCES INC. All rights reserved. No part of this journal may be reproduced in any form without the prior permission of The Canadian Journal of Neurological Sciences. Mailed under Publications Mail Registration number 09824. Postage paid at Calgary, Mailed under Publications Mail Registration number 09824. Postage paid at Calgary,
Alberta. This journal is indexed by Aquatic Sciences and Fisheries Abstracts, ASCA-
Automatic Subject Citation Alert, Biological Abstracts, Chemical Abstracts, Current Automatic Subject Citation Alert, Biological Abstracts, Chemical Abstracts, Cur
Advances in Ecological Sciences, Current Contents (Clinical Medicine and Life Sciences), Dent. Index, e-psyche, Exerpta Medica, Index Medicus, Industrial Science Review, INIS Atomindex, Inpharma, Journal Watch Neurlogy, International Abstracts in Biological Sciences, Laboratory Hazards Bulletin, Neurosciences Citation Inder,

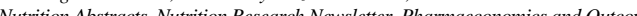
Nutrition Abstracts, Nutrition Research Newsletter, Pharmaeconomics and Outcome
News, Reactions Weekly, Referativnyi Zhurnal, Science Citation Index, Weed Abstracts

Le Journal Canadien des Sciences Neurologiques est publié trimestriellement. L'abonnement annuel est de 80\$C (non-membresau Canada); 80 \$É-U (Etats Unis) et 85 \$É-U (ailleurs); l'abonnement annuel for pour les institutions est de 90 \$C et 85 \$E-U (ailleurs); l'abonnement annuel for pour les institutions est de $90 \$ C$
(non-membres au Canada); 90 \$É-U (Etats Unis) et 95 \$É-U (ailleurs); Internes, résidents, fellows pré et post doctoral voir www.cjns.org pour détails. Copie simple: $22 \$ C$ plus affranchissement et manutention. Toutes les communications et les manuscrits doivent être adressés à Journal Canadien des Sciences Neurologiques, P.O. Box 5456, Station A, Calgary, AB Canada T2H 1X8. Par courrier: 709 - 7015 Macleod Trail SW, Calgary, AB Canada T2H 2K6. Téléphone (403) 229-9575; Fax (403) 229-1661. E-mail journal@cjns.org; Website: www.cjns.org DROITS D’AUTEUR@ 2003: THE CANADIAN JOURNALOP

NEUROLOGICAL SCIENCES INC. Tous droits réservés. Aucune partie de ce Journal ne peut être reproduite, sous quelque forme que ce soit, sans la l'authorisation du Journal Canadien des Sciences Neurologiques. Posté sous registration de postepublications no 09824. Port payé à Calgary, Alberta. Le Journal est cité et indexé dans Aquatic Sciences and Fisheries Abstracts, ASCA - Automatic Subject Citation Alert, Biological Abstracts, Chemical Abstracts, Current Advances in Ecological Sciences, Current Contents (Clinical Medicine and Life Sciences), Dent. Index, e-psyche, Exerpta Medica, Index Medicus, Industrial Science Review, INIS Atomindex, Exerpta Medica, Index Medicus, Industrial Science Review, INIS Atomindex,
Inpharma, Journal Watch Neurology, International Abstracts in Biological Sciences, Inpharma, Journal Watch Neurology, International Abstracts in Biological Sciences,
Laboratory Hazards Bulletin, Neurosciences Citation Index, Nutrition Abstracts, Nutrition Research Newsletter, Pharmaeconomics and Outcome News, Reactions Weekly, Referativnyi Zhurnal, Science Citation Index, Weed Abstracts.

Advertising representative/Représentant de publicité: Sally Gregg, Canadian Journal of Neurological Sciences 709 - 7015 Macleod Trail SW, Calgary, AB Canada T2H 2K6 Tel (403) 229-9575 Fax (403) 229-1661

E-mail: journal@cjns.org; Web Site: www.cjns.org

Printer/Imprimeur:

Sundog Printing Limited, 1311 Ninth Avenue SW, Calgary, Alberta T3C $0 \mathrm{H} 9$

ISSN 0317 - 1671 


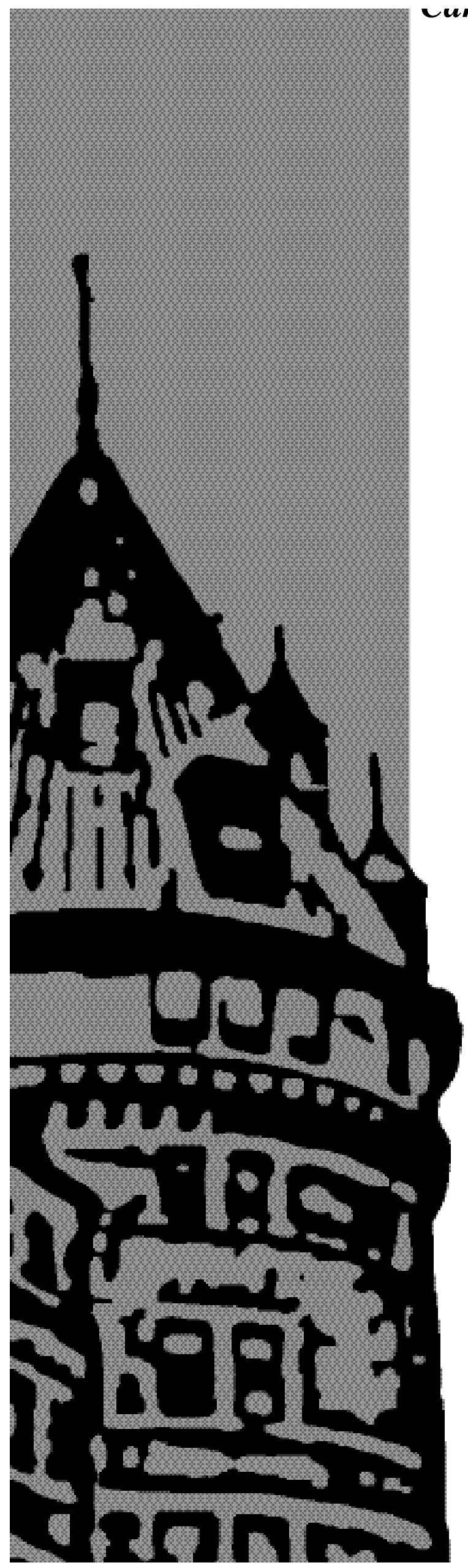

\title{
38th Annual Scientific Meeting
}

\author{
June 17-21, 2003 \\ Quebec City Convention Centre
}

\section{Scientific Program}

\author{
(Subject to Change)
}

\author{
Tuesday, June 17, 2003 \\ Pre-Congress Courses \\ 08:00-17:30 Neurobiology Review Course \\ 09:00-16:00 5th Annual ALS Strategies for Quality Life/Quality Care \\ 18:00-21:00 Movement Disorders Video Session
}

\section{Wednesday, June 18th, 2003 \\ Course Da y}

07:30-17:00 Controversies in Spinal Neurosurgery

08:00-17:30 Epilepsy Review and Update Course

08:00-17:30 Clinical Neurology of Headache 2003

08:00-12:00 Neuroanatomy Review Course

08:00-12:00 Muscle Diseases 2003:Floppiness,Cramps,and Inflammation

13:30-17:30 The CSCN EEG Exam and the New Canadian EEG Guidelines Course

13:30-17:30 Brain Tumor Course

18:00-20:00 Welcome Reception

\section{Thursday, June 19, 2003}

08:30-10:30 Neurophysiologic Applications in Neuroscience

11:00-19:00 Poster session

11:00-13:00 Platform sessions

14:30-16:00 Platform sessions

16:00-17:30 Grand Rounds

17:30-19:00 Special Poster Viewing

\section{Friday, June 20, 2003}

08:30-10:30 New De velopments in Neuropharmacolo gy

11:00-13:00 Platform sessions

11:00-15:00 Poster session

14:30-16:30 Beyond Alzheimer's Disease:The Non-Alzheimer's Dementias Mini-Symposium

14:30-16:30 Case Histories in Neurocritical Care:Mini Symposium

14:30-16:30 What's New in Neurosurgery? Mini Symposium

14:30-16:30 Myasthenia Gravis Mini-Symposium

19:30 Quebecois Soirée

Saturday, June 21, 2003

08:00-17:30 Multiple Sclerosis Symposium 08:00-17:30 Interventional Advances in Neurovascular Disease

08:00-17:30 Child Neurology

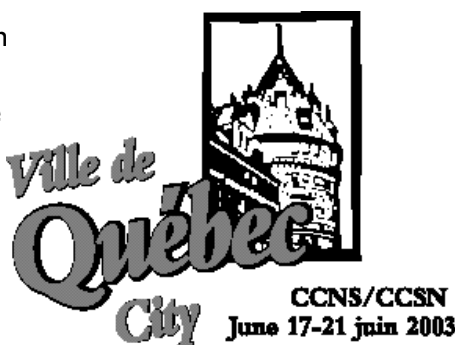




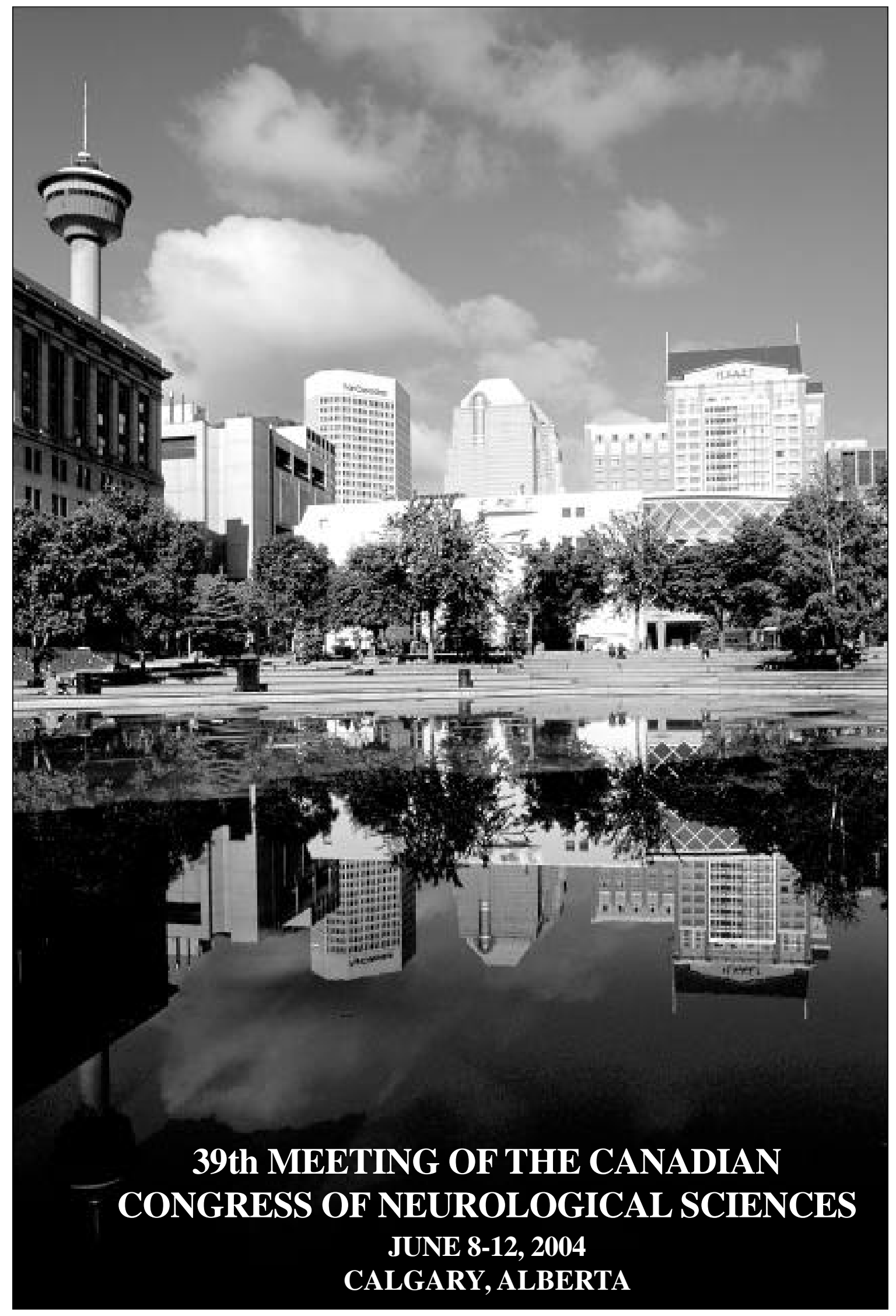




\title{
38TH MEETING OF THE CANADIAN CONGRESS OF NEUROLOGICAL SCIENCES

\author{
JUNE 17-21, 2003 \\ QUEBEC CITY, QUEBEC
}

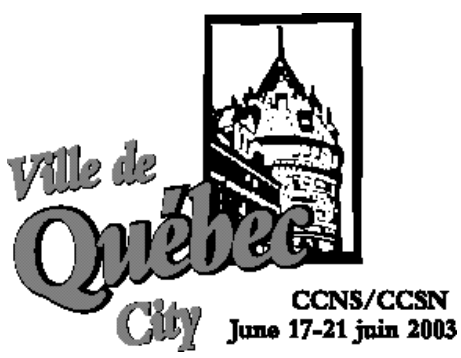

\begin{abstract}
S
Society Prize Presentations

Canadian Neurological Society - Frances McNaughton Memorial Prize

Canadian Association of Child Neurology - President's Prize

Canadian Society of Clinical Neurophysiologists - Herbert Jasper Prize

Canadian Neurosurgical Society - K.G. McKenzie Prize in Clinical Neuroscience Research

Canadian Neurosurgical Society - K.G. McKenzie Prize in Basic Neuroscience Research
\end{abstract}

\section{Platform Presentations}

Thursday June 19, 2003
Friday June 20, 2003
A. Cerebrovascular
A-01 to A-08
B. Neuro-oncology
B-01 to B-08
C. Child Neurology
C-01 to C-08
D. General Neurology I
D-01 to D-08
E. Spine
E-01 to E-06
F. Stroke
F-01 to F-06
G. Neuromuscular
G-01 to G-05
H. Epilepsy
$\mathrm{H}-01$ to $\mathrm{H}-06$

I. General Neurosurgery I

$\mathrm{I}-01$ to $\mathrm{I}-08$

J. Multiple Sclerosis/Dementia

$\mathrm{J}-01$ to $\mathrm{J}-08$

K. Neurophysiology.

$\mathrm{K}-01$ to $\mathrm{K}-08$

L. General Neurology II

$\mathrm{L}-01$ to $\mathrm{L}-07$ M-01 to M-07

\section{Poster Presentations}

\section{Thursday June 19, 2003 and Friday June 22, 2003}

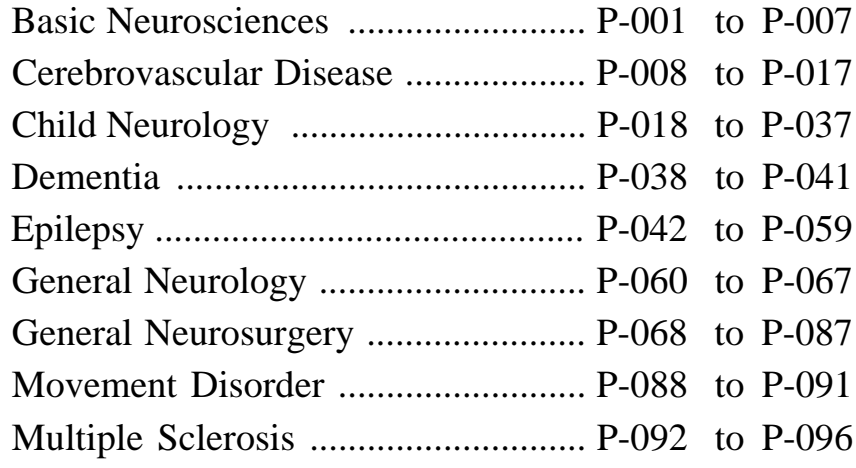

Neuro-oncology P-097 to P-118

Neuro-ophthalmology

$\mathrm{P}-119$ to $\mathrm{P}-121$

Neuro-imaging

$\mathrm{P}-122$ to $\mathrm{P}-125$

Neuromuscular Disease P-126 to P-131

Neurophysiology

$\mathrm{P}-132$

Pediatric Neurosurgery

$\mathrm{P}-133$ to $\mathrm{P}-139$

Spine

$\mathrm{P}-140$ to $\mathrm{P}-145$

Stroke

$\mathrm{P}-146$ to $\mathrm{P}-153$

Vascular Neurosurgery

$\mathrm{P}-154$ to $\mathrm{P}-156$ 


\section{Society Prize Papers}

\section{Frances McNaughton Memorial Prize - Canadian Neurological SOCIETY}

\section{High field functional MRI analysis of interictal discharges in malformations of cortical development: role of surround inhibition and subcortical structures}

\section{P Federico*, J Archer, D Abbott, G Jackson, (Melbourne, Australia)}

Background: Malformations of cortical development (MCD) frequently cause intractable epilepsy associated with an unfavourable postoperative outcome. This may relate to incomplete resection or presence of secondary, unidentified areas contributing to interictal or ictal discharges. We report on how functional MRI analysis of interictal discharges can implicate widespread areas in generating the discharges in MCD.

Methods: Seven patients with MCD and intractable seizures were studied using functional MRI at 3 tesla with concurrent EEG. We measured blood oxygen level dependent (BOLD) signal increases and decreases associated with interictal discharges.

Results: All patients showed BOLD signal increases within the lesion and/or decreases surrounding the lesion (ie. surround inhibition). BOLD signal changes were also seen at distant cortical sites in five patients. In addition, significant BOLD signal changes were seen in the basal ganglia, reticular formation, or thalamus in four patients.

Conclusions: High field functional MRI showed spike-associated BOLD changes in all patients with MCD. These discharges may be generated by activation within the lesion and loss of surround inhibition (disinhibition). Finally, distant cortico-subcortical circuits may play a role in the genesis of these discharges. These observations may also explain why cortically-based surgical resections are often associated with a poor outcome in MCD.

\section{The President's Prize - Canadian Association of Child Neurology}

\author{
Multiple sclerosis versus acute disseminated encephalomyelitis \\ in childhood \\ Steven David Brass*, Zografos Caramanos, Carlos Santos, \\ Marie-Emmanuelle Dilenge, Yves Lapierre, Bernard Rosenblatt \\ (Montreal, QC)
}

Objective: To compare and contrast the initial presenting clinical and laboratory findings of either acute disseminated encephalomyelitis (ADEM) or the first attack of multiple sclerosis (MS) in the pediatric population.

Methods: A retrospective review of the medical records was conducted of all children $<17$ years old who presented to the Montreal Neurological Hospital or Montreal Children's Hospital with either the diagnosis of ADEM or MS between 1987 and 2001.

Results: Seventeen cases of clinically definite MS [seven female; mean years of age $( \pm \mathrm{SD})=12.4 \pm 4.5]$ and seven cases of ADEM [3 female; $8.7 \pm 3.8$ ] were reviewed. A viral infection within 28 days prior to symptom-onset preceded $57 \%$ of ADEM cases but only $18 \%$ of MS. Systemic and non-focal neurological symptoms were more commonly seen in ADEM than in MS: fever (43 vs 6\%), headache ( 57 vs $24 \%$ ), fatigue ( 71 vs $29 \%$ ), vomiting ( 57 vs $0 \%$ ) and encephalopathy (71 vs 6\%). T2-weighted (T2-w) white matter magnetic resonance imaging (MRI) lesions were more common in the corpus callosum (64 vs 17\%) and the periventricular area (91 vs $50 \%$ ) in MS versus ADEM . Electroencephalographic abnormalities ranging from mild to severe disturbance in background were more common in ADEM than MS (80 vs $29 \%$ ). Oligoclonal bands were present in 8/12 MS patients tested versus 0/3 ADEM patients. Of the 3/7 ADEM patients who underwent follow-up, all had normal neurological exam. All 17/17 MS patients who were initially labeled as first attack of MS had a second attack with $81 \%$ of MS patients having Expanded Disability Status Scale Score (EDSS) <4, 6\% having an EDSS $4 \leq 6$, and $13 \%$ with EDSS >6. Conclusion: Although final diagnosis can only be confirmed at follow-up, these results suggest that ADEM and MS can be differentiated to some degree based on clinical and investigational data at initial presentation. This is important because, unlike ADEM, a proportion of pediatric MS cases will develop a significant burden of disease before their third decade and should be studied more intensively in light of evolving therapies. 


\section{Society Prize Papers}

\section{Herbert Jasper Prize - Canadian Society OF Clinical NEUROPHYSIOLOGISTS}

\section{Involvement of the human subthalamic nucleus in movement preparation}

Guillermo Paradiso*, Jean A Saint-Cyr, Andres M Lozano, Anthony E Lang, Robert Chen (Toronto, ON)

Background: Although it has long been recognized that the basal ganglia play a major role in motor control, its precise functions remain unclear. Since patients with Parkinson's disease (PD) had difficulties initiating movement, the basal ganglia may be involved in movement preparation. The cortical areas involved in movement preparation include the supplementary motor area (SMA) and the primary motor cortex. The subthalamic nucleus (STN) is particularly suited to play a role in movement preparation, since it receives direct input from the SMAthrough the cortico-subthalamic pathway, and is part of the indirect cortico-basal ganglia thalamocortical circuit.

Methods: Taking advantage of the electrodes surgically implanted in the STN for deep brain stimulation (DBS) treatment in 13 PD patients, we recorded from the DBS electrodes and the scalp simultaneously, with the aim of assessing electrical changes preceding voluntary movement. The patients performed self-paced wrist extension movements. We analyzed cortical and STN activity four seconds before and one second after movement onset.

Results: Scalp recordings showed a slow, negative movementrelated potential (MRP) in all patients studied (mean onset $1690 \pm$ $336 \mathrm{~ms}$ before EMG onset). Bipolar STN recordings showed MRPs in 11 out of 13 patients. The STN activity occurred with both ipsilateral and contralateral hand movement and consisted of two potentials. The early potential began at $2586 \pm 625 \mathrm{~ms}$ for the contralateral side and $2351 \pm 574 \mathrm{~ms}$ for the ipsilateral side before EMG onset. The late potential began at $562 \pm 160 \mathrm{~ms}$ for the contralateral side and $449 \pm 106 \mathrm{~ms}$ for the ipsilateral side before EMG onset. The onset of the STN-MRP was significantly earlier than that of cortical MRP.

Conclusions: These findings suggested that the STN is active before movement onset and basal ganglia circuits are involved early in movement preparation in humans.

\section{The K.G. McKenzie Prize in Clinical Neuroscience Research - Canadian NeUrosurgical Society}

\author{
Jugular bulb oximetry in prediction of cerebral vasospasm \\ following aneurysmal subarachnoid hemorrhage
}

N Heran*, S Hentschel, B Toyota, (Vancouver, BC)

Background: Cerebral vasospasm adversely impacts the outcome of those suffering aneurysmal subarachnoid hemorrhage (SAH). Prediction of vasospasm could improve outcomes. We hypothesized that preclinical vasospasm would be heralded by an increase in cerebral oxygen extractions $\left(\mathrm{AVDO}_{2}\right)$ which could be detected by jugular bulb oximetry. A pilot study was conducted to address this hypothesis.

Methods: 14 consecutive consenting patients with aneurysmal SAH, undergoing early surgery, were entered into the study. Four patients were withdrawn from the study secondary to failure of catheters or religious belief. At the time of craniotomy, a jugular bulb catheter was placed. Post-operatively, arterial and jugular bulb blood samples were taken every 12 hours to calculate $\mathrm{AVDO}_{2}$. As this was an observational study, no change in management occurred based on measurements.

Results: Four of 10 patients had clinical vasospasm. These patients had a significant rise in $\mathrm{AVDO}_{2}$ approximately one day prior to the onset of neurologic deficits $(\mathrm{P}<0.001)$. Symptoms resolved along with a significant improvement in $\mathrm{AVDO}_{2}$ on instituting $\mathrm{HHH}$ therapy in these patients. The six patients who did not exhibit clinical vasospasm did not demonstrate significant rise in $\mathrm{AVDO}_{2}$.

Conclusions: Jugular bulb oximetry is simple and cost effective. Increases in $\mathrm{AVDO}_{2}$ using this technique were predictive of clinically evident vasospasm in the subsequent hours to days. This investigation supports a larger study to assess the utility of jugular bulb oximetry in predicting vasospasm in aneurysmal SAH. 


\section{K.G. McKenzie Prizes in Basic Neuroscience Research - Canadian NeUrosurgical SocieTy}

\section{Novel synthetic grafts promote axonal regeneration and functional recovery after spinal cord transection}

E Tsai*, R DeMarchi, P Dalton, M Shoichet, C Tator, (Toronto, $O N)$

Background: We previously reported that a novel tubular device, composed of a rigid or semi-rigid formulation of poly (2hydroxyethyl methacrylate) (PHEMA), is biocompatible after spinal cord injury. In this study, we assess the ability of PHEMA tubes to promote axonal regeneration.

Methods: Adult Sprague Dawley rats underwent complete spinal cord transection at $\mathrm{T} 8$ and repair with rigid or semi-rigid PHEMA tubes. The cord stumps were inserted into the tube, fibrin glue was applied to the cord-tube interface, and Preclude ${ }^{\mathrm{TM}}$ membrane used for duraplasty. Controls underwent cord transection alone. Retrograde axonal tracing with Fluoro-Gold was used to demonstrate axons that regenerated through the tube. At 2, 4, and 8 weeks, animals were perfused and underwent immunohistochemical evaluation.

Results: In both tube types, neurofilament stained axons and calcitonin gene related peptide stained sensory axons were visualized within the tissue bridge. However, only in the animals with rigid tubes were retrogradely labeled Fluoro-Gold neurons found in brainstem motor nuclei.

Conclusions: Both tube types promote axonal regeneration, however, only the rigid PHEMAtubes promote axon regeneration of brainstem motor nuclei after spinal cord transaction. The use of PHEMAtubes is a novel therapeutic strategy that may hold promise for those with spinal cord injury.
Analysing tumor vascularity with use of small animal imaging

G Zadeh*, B Qian, N Bock, A Guha, (Toronto, ON)

Introduction: Angiopoietins are known to regulate angiogenesis, however, their role in astrocytoma angiogenesis is not known. Our study focuses on determining their involvement in astrocytoma vascularization using imaging studies.

Methods: Astrocytoma cell line (U87) were transfected to overexpress Ang1 or Ang2 and each injected into six mice intracranially using a stereotactic frame, with U87 parental cells being used as control. Tumor latency, growth and vascularity was monitored weekly over a six week period using T1/T2/T1 with gadolinium sequences in a 7 Tesla small animal MRI coupled with CTangiograms at the end of the study.

Results: Temporal course of tumor growth demonstrated a faster rate of tumor growth for Ang1 upregulated tumors compared to control. Tumors with Ang2 upregulation demonstrated a higher extent of tumor vascularity and enhancement that was confirmed by CT-angiogram. The latency period between the three groups remained unchanged.

Conclusions: This is the first report of intracranial astrocytoma models that have been followed by MRI combined with CT angiogram, illustrating that small animal imaging can be used effectively to follow the temporal growth of astrocytoma models coupled with the angiogenic progression of the tumors. The angiogenic data will provide dynamic validity to the current methods of analyzing tumor vascularity. 


\section{Platform Presentations}

\section{Cerebrovascular Surgery}

\section{A-01}

\section{Jugular bulb oximetry in prediction of cerebral vasospasm following aneurysmal subarachnoid hemorrhage}

N Heran*, S Hentschel, B Toyota, (Vancouver, BC)

Winner of the Canadian Neurosurgical Society's K.G. McKenzie Prize in Clinical Neuroscience Research (see page 10).

\section{A-02}

Single center analysis of outcomes in North American patients enrolled in ISAT

H Jain*, W Montanera, KG ter Brugge, MC Wallace, $R$ Willinsky, M Tymianski, (Toronto, ON)

Introduction: The ISAT trial was a multi-center randomized evaluation of the efficacy of surgery vs GDC embolisation of ruptured aneurysms. We evaluated our results to determine the applicability of ISAT to North American patients

Methods: 61 patients were randomized into ISAT during the trial. The prospectively collected data sheets were analyzed after the trial was terminated. Clinical outcome was determined according to the Modified Rankin score at last follow-up. Treatment mortalities, cross-overs and re-haemorrhage rates were noted. The effect of treatment modality on outcome was determined by chi-square analysis.

Results: 31 and 30 patients were randomized to surgery and GDC coiling, respectively. No surgical patients re-haemorrhaged, whereas two GDC coiled patients suffered lethal re-bleeds. There were two cross-overs from GDC coiling to surgery. At last follow-up, 12 surgical and 19 GDC patients had Rankin scores 0 or 1 , and 7 surgical and 5 GDC patients had Rankin scores of 2 . There was no statistical difference in outcomes between the treatment groups (chisquare=2.779, $\mathrm{p}=0.096$ for Rankin 0 and 1)

Conclusions: GDC coiling and surgery each have roles in the management of ruptured intracranial aneurysms. Re-haemorrhage may be higher amongst patients treated with GDC coils. Long-term follow-up is needed to determine aneurysm recurrence, rehaemorrhage rates and subsequent clinical outcomes.

\section{A-03}

Cranial CT angiography as primary diagnostic measure in patients with aneurysmal subarachnoid hemorrhage

M Kass*, AM Kaufmann, B McClarty, M West, (Winnipeg, MB)

Introduction: We use computed tomographic angiography (CTA) as a fast, safe, accurate, and relatively inexpensive method of diagnosing ruptured cerebral aneurysms.

Methods: 58 patients with a SAH had a CTA and those with negative CTAs also had a digital subtraction angiogram (DSA).

Results: Two patients had no CTAs because of technical difficulties; both of whom had aneurysms on subsequent DSAs. Of the remaining 56 patients, CTA found 49 aneurysms in 44 patients. CTA was negative for aneurysm in 12 patients; all were negative on subsequent DSA as well. The average aneurysm size was $10.25 \mathrm{~mm}$ (range $2 \mathrm{~mm}-40 \mathrm{~mm}$ ). 37 patients went straight to surgery following CTA. The sensitivity of CTA was $100 \%$ for ruptured aneurysms and $98 \%$ for all aneurysms. The specificity was $100 \%$ for both ruptured and unruptured aneurysms. Median time from presentation at emergency triage to CTA was 86 minutes; triage to surgery was 382 minutes.

Conclusions: The CTA is a safe, accurate, and inexpensive method of diagnosing intracranial aneurysms in patients with SAH. In our institution, we always do the CTA for SAH and only do DSAs when the CTA is negative or an arteriovenous malformation is suspected.

\section{A-04}

Intracranial hemorrhage and infectious intracranial aneurysm in bacterial endocarditis

A Parr*, $M$ West, (Winnipeg, MB)

Background: Intracranial hemorrhage (ICH) is a life-threatening complication occurring in 5\% of patients with infective endocarditis. One treatable cause is infective intracranial aneurysm reported in $2 \%$ to $10 \%$ of endocarditis patients, with a mortality of $80 \%$ at rupture. The purpose of this study is to describe a subgroup of patients with endocarditis who have intracranial hemorrhage and/or infectious intracranial aneurysm.

Methods: A retrospective chart review was performed over six years.

Results: A total of 151 cases of bacterial endocarditis were identified from two centres. Intracranial complications were identified in $40(26 \%)$ patients. Hemorrhage was identified in 18 $(11 \%)$ patients. Of these, three $(2 \%)$ cases were angiographically identified aneurysm. One $(0.7 \%)$ case of unruptured infective aneurysm was also identified. The patients who developed ICH or aneurysm were more likely to develop focal signs or symptoms in hospital. These patients also had Staphylococcus aureus or mitral involvement more frequently. No patient with isolated right heart disease developed ICH or aneurysm. ICH patients had poorer outcomes.

Conclusions: This study highlights the neurologic sequelae of infective endocarditis, specifically ICH and aneurysm, in order to increase awareness of these diagnoses. Further characteristics of these patients and their management will be discussed.

\section{A-05}

A cohort study of intraventricularthrombolysis with rt-PA for aneurysmal intraventricular hemorrhage

J Max Findlay*, Michael J Jacka, (Edmonton, AB)

Background: Fibrinolytic agents have been administered through external ventricular drains to treat intraventricular hemorrhage (IVH), the goals being to accelerate clot clearance, prevent catheter obstruction and help control ICP. We compared these variables in a 
group of aneurysm patients treated by one surgeon who routinely used intraventricular rt-PA for obstructive hematocephalus, to a group of similar patients treated by other surgeons who did not.

Methods: Patients included were those with ruptured cerebral aneurysms and hemorrhage into at least three ventricles causing ventriculomegaly. In the treatment group rt-PA (4 mg per daily dose) was given through a ventricular drain following aneurysm repair. The ventricular system was considered "opened" when all ventricles were patent and normal in size on CT scan.

Results: The mean number of days to ventricular opening was 3.9 (SD 1.2) for the 22 patients treated with rt-PA and 12.2 (SD 4.9) for the nine who did not receive rt-PA $(\mathrm{P}=0.0009)$, and the mean ICP $(\mathrm{mm} \mathrm{Hg}) 48$ hours posthemorrhage was 9.0 (SD 3.9) for the rt-PA group and 14.1 (SD 5.9) for the no rt-PA group ( $\mathrm{P}=0.008)$. Ventricular catheter replacement was required in one rt-PA patient and four patients who did not receive rt-PA $(\mathrm{P}=0.016)$, and VP shunts were placed in $4 \mathrm{rt}-\mathrm{PA}$ patients and three patients who did not receive rt-PA $(\mathrm{P}=0.23)$.

Conclusions: Intraventricular thrombolysis with rt-PA appears to speed clearance of aneurysmal IVH, help normalize ICP and reduce ventricular catheter obstruction with little risk. A randomized trial is needed to confirm these findings and determine if treatment improves outcome.

\section{A-06}

\section{Lateral tentorial sinus: delineation with contrast-enhanced MRI}

Mehran Midia*, (Halifax, NS); Zinat Miabi, (Tabriz, Iran); Ramin Midia, (Springfield, IL); William Maloney, Robert Vandorpe, (Halifax, NS)

Background: Lateral tentorial sinuses are formed by convergence of veins from the basal and lateral surface of the temporal and occipital lobes. The purpose of this is study is to evaluate the visualization and variability of this venous tributary by MRI.

Methods: Conventional contrast-enhanced MR images of the 55 consecutive adult patients who had no abnormality of lateral tentorial sinuses were retrospectively reviewed. The incidence of detection of these sinuses, their branches, and variations were recorded.

Results: With conventional contrast-enhanced T-1 weighted imaging right lateral tentorial sinus was detected in 50 and left in 54 patients. Of these on the right side 16 type I, 12 type II, 17 type III, and 10 were indeterminate. Similarly 14 type I, 10 type II, 20 type III and 11 were indeterminate on the left side. Right and left vein of Laabe were identified in 47 patient. Right veins of Laabe was draining into the tentorial sinus in 23 patient and left was draining similarly in 30 patients. Incidence of detection of lateral tentorial sinuses branches are also reported.

Conclusions: An understanding of the presence and diversity of this venous structure and its configuration would be helpful in interpreting $\mathrm{MR}$ imaging studies and perhaps avoiding complications during lateral cranial base surgery.
A-07

\section{Radiosurgical retreatment of brain arteriovenous malformations (AVMs)}

J Mirza-Aghazadeh, M Tsao, D Scora, M Schwartz*, (Toronto, ON)

Background: Stereotactic radiosurgery (SRS) causes obliteration of most AVMs after a latency period of about three years with a complication rate of about 5\%. Protection from hemorrhage requires complete obliteration. Digital subtraction angiography (DSA) is the most sensitive detector of small AVM remnants that constitute a threat of hemorrhage. When SRS reduces the size of, but fails to obliterate an AVM after three years, a second treatment may be offered.

Methods: Prospective database review.

Results: Of more than 250 radiosurgery treatments for brain AVMs, 15 were a second treatment for residual AVM. Twelve were originally treated by us, and three were treated elsewhere by gamma knife, linear accelerator and proton beam units, respectively. One patient is two years postsecond treatment. Of 12 patients with DSA results, two failures: an elongated malformation, not entirely encompassed by the treatment volume at the first treatment, and a second small AVM remnant excised after the second SRS. Ten had complete obliteration of the AVM. Three patients suffered a new neurological deficit. Two patients, in remote cities, lack follow-up DSA, which we are trying to arrange.

Conclusions: Prior radiosurgical treatment of an AVM confers greater susceptibility to obliteration, and perhaps to radiation injury.

\section{A-08}

A discriminative prediction model for neurological outcome for patients undergoing surgery of brain arteriovenous malformations

\section{J Spears*, M Tymianski, K ter Brugge, M Moosavian, F Gentili, MC Wallace, (Toronto, ON)}

Introduction: Several radiographic factors have been implicated in the development of new neurological deficits following surgery of brain AVMs including: size, eloquence, deep venous drainage (Spetzler-Martin Grading System), deep arterial perforator supply, diffuse nidus morphology, deep white matter configuration and associated aneurysms. The specific aim of this project was to develop a prediction model for neurological outcome following surgery of brain AVMs based on the seven aforementioned variables.

Methods: A cohort study consisting of 175 patients was performed based on prospectively collected data from the University of Toronto AVM Study Group's database, comprised of all consecutively treated surgical patients possessing a brain AVM within a single, tertiary care centre during a 12 year period. Each predictor variable was introduced into a logistic regression model to generate a prediction rule for neurological outcome following surgery. Primary outcome measures included the incidence of new neurological deficits and poor early functional outcomes following surgery. Secondary outcomes included the presence of persistent neurological deficits at least one year following surgery and permanently poor functional outcomes.

Results: The predictors influencing the incidence of new disabling neurological deficits following surgery included: 
Eloquence $\{\mathrm{OR}=4.183[1.411,12.404]\}$, Diffuse Nidus Morphology $\{\mathrm{OR}=3.122[1.281,7.606]\}$, Deep Venous Drainage $\{\mathrm{OR}=2.363[1.024,5.453]\}$ and Size $\{\mathrm{OR}=1.362[0.572,3.243]\}$. Based on these odds ratios a stratified risk score has been proposed for the development of poor neurological outcomes following surgery as follows: Score 0-2 $=1.9 \%, 3-5=14.9 \%, 6-8=32.8 \%, 9$ $10=51.5 \%($ Chi-square $=34.1, \mathrm{p}<0.0001)$, [Eloquence $=4$ points, Diffuse Nidus Morphology $=3$ points, Deep Venous Drainage $=2$ points and Size greater than $3 \mathrm{~cm}=1$ point].

Conclusions: We propose a simple, discriminative, risk stratification score for neurological outcome in AVM surgery with relative weights assigned to each significant predictor reflecting the relative influence of each predictor variable on outcome.

\section{NeURO-OnCOLOGY}

\section{B-01}

\section{Analysing tumor vascularity with use of small animal imaging \\ G Zadeh*, B Qian, N Bock, A Guha, (Toronto, ON)}

Co-winner of the Canadian Neurosurgical Society's K.G. McKenzie Prize in Basic Neuroscience Research (see page 11)

\section{B-02}

\section{Intraoperative MRI at $\mathbf{1 . 5 T}$}

GR Sutherland*, PB McBeth, DF Louw, (Calgary, AB)

Background: The history of neurosurgery has largely depended on its increasing ability to image the brain. The purpose of our project was to design, construct and evaluate a mobile $1.5 \mathrm{~T}$ system in the operating room.

Methods: Three hundred and eighty-six patients were operated on under iMRI guidance. They had various pathologies, including tumors, epilepsy, vascular and spinal disorders. A suite of standard imaging techniques were available, including T1, T2, FLARE, MRA, diffusion-weighted or perfusion images.

Results: We have now operated on 386 patients under iMRI guidance. It was readily evident that a substantial portion of lowgrade glioma patients (76) benefited from this technique: approximately one quarter demonstrated unsuspected residual tumor on interdissection images. Quality assurance images both confirm and standardize surgical objectives, which should enhance the robustness of future glioma surgery outcomes trials. Half the pituitary adenoma patients had significant tumor remnants detected intra-operatively, necessitating further resection. Epilepsy (33) procedures have been enhanced over half the time, as surgeons can now verify ablation of the amygdala and other epileptogenic detonators before wound closure (17).

Conclusions: We have shown that iMRI at $1.5 \mathrm{~T}$ supplements standard surgical technique by enhancing accuracy, reducing invasiveness and the need for redo surgery to remove residual tumor.
B-03

\section{Transgenic mouse models of astrocytomas}

H Ding, A Nagy, N Lau, P Shannon, A Guha*, (Toronto, ON)

Introduction: Gliomas are the most common primary CNS tumor and remain incurable. Understanding the molecular pathogenesis will hopefully lead to novel effective biological therapies, further increasing the need for small animal models that better replicate human gliomas.

Methods: Embryonic stem cell transgenesis was used to create mouse models of astrocytomas and oligodendrogliomas. Glial specific GFAP promoter was used to express activated Ha-p21-Ras and EGFRvIII (mutant EGFR), which we have identified as common molecular aberrations in human gliomas.

Results: Expression of GFAP:Ha-p21-Ras resulted in malignant astrocytomas in $95 \%$ of the mice, with a mean survival of $12-14$ wks. The astrocytomas are pathologically and molecularly similar to human counterparts. Expression of GFAP:EGFRvIII by itself did not cause tumor formation, in keeping with EGFRvIII being a progression rather than a initiation factor in human gliomas. However, when mated to the GFAP:Ha-p21-Ras mice there was tumor potentiation, with mean survival of only 2-3wks. Furthermore, there was a switch in tumor type with the double transgenics having predominant oligodendroglioma features.

Conclusions: We have developed a spontaneous infiltrating glioma model, that can serve as better preclinical models to evaluate biological therapies, in addition to being used to increase our knowledge of the molecular pathogenesis of gliomas.

\section{B-04}

TGF- $\beta$ expression in a series of glioma cell lines (F98, u373, u118, u87)

R Adams*, D Fortin, (Sherbrooke, QC)

Background: The development of primary brain tumors involves a cascade of molecular events leading to deregulated cell growth. A number of growth factors have been found to be over-expressed in high-grade glial lesions and considered to be involved in tumorigenesis. Transforming growth factor beta (TGF- $\beta$ ) is one such factor. TGF- $\beta$ is usually expressed in inactive form that requires post-translational activation. Gliomas can secrete TGF- $\beta$ directly in active form. We undertook the characterization of total and active TGF- $\beta$ expression in different glial cell lines.

Methods: The cell lines were propagated by standard technique. The supernatant of each cell lines at confluence was submitted to an ELISAessay to dose the total and active TGF- $\beta$.

Results: TGF- $\beta$ was expressed by each cell line in both the inactive and active fraction. F98 cell line expressed the most significant quantitative amount of total TGF- $\beta 1$ (3439 pg/ml, active fraction 16\%). However, interestingly, the $\mathrm{u}-373$ cell line expressed smaller amount $(302 \mathrm{pg} / \mathrm{ml})$, but larger ratio of active TGF- $\beta 1$ (32\%).

Conclusions: In the future we intend to suppress the expression of the active fraction of TGF- $\beta$ as a potential strategy to suppress tumor growth. The F98 cell line is a good model to test this approach. 
B-05

\section{Downregulated in renal cell carcinoma, a tumor suppressor} that enhances glioma cell Invasion

\section{K Petrecca, J Wang, A Angers-Loustau, RF Del Maestro,} (Montreal, $Q C$ )

Malignant glial cell invasion represents a major obstacle in the treatment of glial-derived brain tumors. In order to uncover molecular mechanisms that play a role in invasion, we developed a novel functional screening assay to identify genetic determinants of motility and invasion. To do so a U251 glioma cell line was transduced with a normal human adult brain cDNA library containing $\sim 1 \times 10^{7}$ independent clones using retroviral gene transfer. Cellular spheroids were generated from these cells and implanted into a collagen type I gel. Hyperinvasive cells were singly dissected from the matrix and their cDNA inserts were identified. One candidate cDNA insert encoded full-length downregulated in renal cell carcinoma (drr-1), a putative tumor suppressor. Heterogeneous expression reveals a restricted drr-1 subcellular distribution with protein expression at the leading edge of lamellipodia, along the reorganizing cytoskeleton and in the nucleus. Importantly, spheroids generated from cells stably expressing drr-1 showed a $240 \%$ increase in invasiveness. Moreover, Map1a, an inducer of rapid tubulin polymerization, was identified as a drr1associated protein using yeast 2-hybrid screening. Taken together, these data implicate drr-1 as a mediator of cytoskeletal reorganization and lamellipodia formation that enhances glial cell invasion.

\section{B-06}

\section{Identification of a cancer stem cell in human brain tumours}

Sheila K Singh*, Ian D Clarke, Mizuhiko Terasaki, Victoria E Bonn, Cynthia Hawkins, Jeremy Squire, Peter B Dirks (Toronto, Canada)

Most current research on human brain tumours is focussed on the molecular and cellular analysis of the bulk tumour mass. However, there is overwhelming evidence in some malignancies the tumour clone is heterogeneous with respect to proliferation and differentiation. In human leukemia, the tumour clone is organized as a hierarchy that originates from rare leukemic stem cells that possess extensive proliferative and selfrenewal potential and are responsible for maintaining the tumour clone. We report here the identification and purification of a cancer stem cell from human brain tumours of different phenotypes that possess a marked capacity for proliferation, self-renewal and differentiation. The increased selfrenewal capacity of the brain tumour stem cell (BTSC) was highest from the most aggressive clinical samples of medulloblastoma compared with low grade gliomas. The BTSC was exclusively isolated with the cell fraction expressing the neural stem cell surface marker CD133. These CD133+ cells could differentiate in culture into tumour cells that phenotypically resembled the tumour from the patient, establishing that the brain tumour clone, like leukemia, is organized as a hierarchy. The identification of a BTSC provides a powerful tool to investigate the tumourigenic process in the central nervous system and to develop therapies targeted to the BTSC.
B-07

GBM xenografts as a model for studying the amplification of EGFR in gliomas

A Pandita*, G Zadeh, BP Qian, (Toronto, ON); M Schroeder, CD James, A Guha, (Rochester, MN)

Introduction: Amplification of mutant and/or wild-type EGFR is observed in approximately a third of human glioblastomas (GBM), and is the only gene alteration in GBMs that is selected against when establishing GBM cell lines. The relative lack of EGFR amplified GBM cell lines presents a major obstacle in studying the function and biologic consequences of this gene alteration.

Results: 7/17 xenografts established have EGFR amplification, with the amplification status of only $3 / 7$ xenografts similar to the corresponding primary GBM. Two xenografts gained EGFR amplification, not detected in the original GBMs. An additional two xenografts, showed conversion to exclusive amplification of EGFRvIII while being passaged in mice.

Conclusions: These results suggest that continued in vivo growth of GBMs tends to select for EGFR amplification and preferential propagation of activated EGFR mutants. In all instances, the cell lines lack amplified EGFR, including cases where the corresponding primary tumor showed EGFR amplification. To determine whether EGFR amplification recurs when cell cultures, are returned to an in vivo growth environment, we have injected cultured cells into nude mice. These paired, isogenic GBM xenografts and cell lines will be useful for investigating specific biological and therapeutic response properties that are associated with the expression of EGFR/ EGFRvIII in GBMs.

\section{B-08}

Human dendritic cells primed with glioma or malignant melanoma naturally processed tumour peptides induce cytotoxic antitumour response in vitro

R Kerr*, H Chen, K Petruk, (Edmonton, AB); M Protti, (Milan, Italy)

Introduction: Glioblastomas and melanomas have evolved complex methods to avoid recognition and effective immune system response. Dendritic cells (DCs) are antigen presenting cells capable of strong antigen presentation in the context of MHC and costimulatory molecules. We hypothesize that autologous or HLA-A2 matched DCs primed with naturally processed tumour peptides from malignant gliomas or melanomas can induce CD8+ lymphocyte lysis of target tumour cells in vitro.

Methods: Autologous or HLA-A2 matched DCs were generated from CD14+ monocytes and primed with naturally processed tumour peptides extracted from early explant tumour cultures. Primed DCs were co-cultured with matched CD8+ lymphocytes. CTLs were evaluated for proliferation, IFNy secretion, and target cell lysis.

Results: CTLs demonstrated prolific clonal expansion by 48 hours. One week after admixture, IFN $\gamma$ concentrations for autologous experiments for Ed276Bt, Ed326Bt,and Ed343Mel were 477, 301.5 and 3,593 pg/ml respectively. Cr51 CTL assays using HLA-A2 matched DCs and CTLs against SK24Mel and Ed276Bt at a 1:20 target/effector cell ratio, resulted in $38 \%$ and $58 \%$ target cell lysis respectively at four hours. 
Conclusions: Autologous or HLA-A2 matched DCs primed with glioma or melanoma peptide extracts induce CD8+ lymphocyte proliferation, activation, and cytotoxic lysis of glioma and melanoma cells in vitro.

\section{Child Neurology}

\section{C-01}

\section{Multiple sclerosis versus acute disseminated encephalomyelitis in childhood}

Steven David Brass*, Zografos Caramanos, Carlos Santos, MarieEmmanuelle Dilenge, Yves Lapierre, Bernard Rosenblatt (Montreal, QC)

Winner of the Canadian Association of Child Neurology's President's Prize - see page 9.

\section{C-02}

\section{Chronic daily headache (CDH) in children and adolescents}

Shashi Seshia*, (Winnipeg, MB)

Background/Objectives: Characterise $\mathrm{CDH}$ in children/adolescents.

Methods: (i) Study design: prospective, observational, sequential. (ii) Setting: private practice pediatric neurology clinic in a Canadian City (Winnipeg). (iii) Data: those referred with headache (HA) between September 1998-December 2001 were entered on data sheets. $\mathrm{CDH}$ was defined as occurrence of at least 15 days with HA/month for at least one month. (iv) Follow-up: three months four years.

Results: 143 (30\%) of 463 referred with HA had CDH with a duration of one month to five years (median: eight months). There were significantly more females $(\mathrm{N}=93)$ than males $(\mathrm{N}=50)$. The age range was 5.5-20 years (median: 13 years). Tension-type features dominated in $91 \%$.Analgesia abuse only occurred in two. Stressors were recognised in $60(42 \%)$, anxiety in $8(6 \%)$, depression in 13 (9\%). CT Scans were done in $31(22 \%)$, and MRI/A in $8(6 \%)$, and were normal. $42(29 \%)$ were treated; $29(20 \%)$ were seen by a psychiatrist; 22 (15\%) were lost to follow-up. 115/remaining 121 (95\%) were headache-free/greatly improved.

Conclusions: (i) $\mathrm{CDH}$ is common in preteens and teens, specially amongst girls, and (ii) a prospective neuropsychiatric approach is necessary for evidenced-based management, since the condition has mental health, social and economic ramifications.

\section{C-03}

Features associated with frequent headaches in young Canadian adolescents

K Gordon*, J Dooley, E Wood, (Halifax, NS)

Objective: To explore the associated factors for frequent headache among young adolescent Canadians.

Methods: We analysed the self-administered questionnaire microdata files of the National Longitudinal Survey of Children and Youth (NLSCY: 1996-1997). 2090 respondents representing 793,100 Canadian youth age 12-13 years were asked how often they had headaches in the previous six months.
Results: 1998/2090 (96\%) responded with $26.6 \%$ of Canadian youth age 12-13 years (95\% CI: 24.2, 28.6) reporting frequent headaches of "about once a week" or more often. Frequent headaches appear to be associated with a plethora of risk factors germane to the life experience of these young adolescents. A few examples of risk factors from the bivariate analysis include: disliking school (OR 1.68), pessimism about the future (OR 3.00), skipping breakfast (OR 1.70), exposure at school to attempted/ committed suicide (OR 1.79), all $\mathrm{p}<.0001$ by Chi-square analysis. A multivariate CART analysis models frequent headaches on a depression scale, a self-esteem scale, and ever having smoked, with $59 \%$ sensitivity and $62 \%$ specificity.

Conclusions: The NLSCY reveals remarkable insights into headache frequency and the life experience of Canada's young adolescents with frequent headaches.

\section{C-04}

Acute disseminated encephalomyelitis in children: evolution of magnetic resonance imaging features and clinical follow-up

L Richer*, B Sinclair, (Edmonton, AB)

Background: Acute disseminated encephalomyelitis (ADEM) is a monophasic demyelinating disorder of the central nervous system. We report nine consecutive pediatric cases.

Methods: Sequential cases of ADEM between Jan, 2000 and Dec 2002 at the Stollery Children's Hospital were included. Systematic chart review was performed and independent review of magnetic resonance imaging (MRI).

Results: Neurological presentation was highly variable with ataxia, myelopathy, bilateral visual loss, seizures, encephalopathy, and hemiparesis. The immediately subcortical white matter showed $\mathrm{T} 2$ prolongation on MRI in five, periventricular white matter in two, and deep gray matter, cerebellum, and brainstem in two. Two also had bilateral optic neuritis and four in total had cerebellar involvement. Follow-up MRI within three to six months was available in seven patients with significant resolution in six. Four had mild neurological sequelae and/or epilepsy and three were normal on follow-up up to 12 months. One patient had persistent MRI abnormalities and later relapsed. One patient was lost to follow-up.

Conclusions: The neurological presentation of ADEM is highly variable. Resolution of MRI abnormalities is an important feature of ADEM and those with persistent MRI abnormalities on follow-up may have a chronic or relapsing demyelinating disease.

\section{C-05}

Informative randomized clinical trials (RCTs) about antiepileptic drug (AED) effectiveness in children are regrettably few

P Camfield*, C Camfield, (Halifax, NS)

Background: RCTs should be the gold standard of treatment for childhood epilepsy management.

Methods: Medline review, excluding primary proof of efficacy trials.

Results: There are only 27 RCTs comparing AED efficacy, exploring therapy for untreatable syndromes or reporting negative effects. We list what is known. In newly treated children, 
carbamazepine, oxcarbazepine, phenytoin, valproic acid, clobazam and phenobarbital have equal efficacy. Phenobarbital may have more side effects than carbamazepine, phenytoin and valproic acid. Carbamazepine may have more effect on memory than phenytoin or valproic acid. Gabapentin is ineffective for childhood absence and benign Rolandic epilepsy. Stiripental has some efficacy in combination with other AEDs for severe myoclonic epilepsy of infancy. For West syndrome, low dose ACTH is as effective as high dose: both are better than oral prednisolone. ACTH is better than vigabatrin except in tuberous sclerosis where vigabatrin is superior to ACTH and oral hydrocortisone. High dose vigabatrin is more effective than low dose. For acute seizures, IM midazolam may act faster than IVdiazepam and rectal lorazepam may be more effective than diazepam. That's it!

Conclusions: Given that $1 \%$ of the children have epilepsy, these results are discouraging. Collaboration for RCTs must become a priority for childhood epilepsy specialists.

\section{C-06}

\section{Neonatal and infant brain death: how often does it occur?}

C Hahn*, L McCarthy, J Hellmann, B Banwell, (Toronto, ON); $S$ Shemie, (Montreal, QC)

Background: The objective of this study was to estimate the incidence of neonatal and infant brain death at a single tertiary children's hospital, in preparation for a future multi-centre study.

Methods: We reviewed all deaths occurring during the year 2000 in an outborn neonatal intensive care unit at The Hospital for Sick Children. Structured chart reviews were conducted for all patients meeting the inclusion criteria of conceptional age at death 36 weeks or greater, and weight at death $2000 \mathrm{~g}$ or greater. Patients were categorized as follows: established brain death, probable brain death (meeting all criteria without formal testing), possible brain death (meeting some criteria, but incomplete documentation) and not brain death (any findings that exclude brain death).

Results: 48 deaths occurred in the NICU during the study period, of which 20 met the inclusion criteria. There were no cases of established brain death, two cases of probable brain death, and two cases of possible brain death. The most common diagnosis among patients with probable and possible brain death was hypoxicischemic encephalopathy.

Conclusions: Based on this review, we estimate the annual incidence of neonatal and infant brain death at this hospital to have been between $4 \%$ and $8 \%$ of NICU deaths.

\section{C-07}

Efficacy of vagal nerve stimulation in refractory epilepsy: the Calgary comprehensive pediatric and adult epilepsy clinic experience

L Hamiwka*, E Wirrell, N Pillay, W Murphy, M Blackman,

$J$ Martini, (Calgary, $A B$ )

Background: Vagal nerve stimulation (VNS) is considered a treatment option for those with refractory epilepsy. Given the associated cost and morbidity, effectiveness of this therapy needs to be established.

Methods: We reviewed all individuals $(\mathrm{n}=27)$ treated with VNS at our clinic. Two adults were excluded (inadequate follow-up).
Results: Twenty-five individuals (12 children; 13 adults at implant) were reviewed and followed for five to 52 months (mean = 25). Eight had generalized epilepsy and 18 had partial epilepsy. All were refractory (mean anticonvulsants; $n=8)$. Only two $(8 \%)$ had favorable outcome (greater than $50 \%$ seizure reduction). Two had a decrease in the number of anticonvulsant medications. The magnet was useful in aborting seizures in 16 of 23 (11 less than 50\% seizures; 5 greater than $50 \%$ seizures; 2 ; no use). Although 14 individuals $(56 \%)$ had improved well being, only $5(26 \%)$ had improved cognition.

Conclusions: VNS was minimally effective for improved seizure control. Use of VNS did not allow for reduction in other medical therapies. A significant number reported a sense of well being which did not correlate with seizures outcome or decrease in medication. An improvement in cognition was reported in $25 \%$, however this is difficult to attribute to VNS without prospective neuropsychological assessments.

\section{C-08}

\section{Establishing an ocular motor laboratory in pediatrics}

M Salman*, J Sharpe, M Eizenman, L Lillakas, M Dennis, $C$ Westall, $M$ Steinbach, (Toronto, ON)

Background: Research in eye movements provides a useful method for studying brain function. Examining and measuring eye movements is useful in localizing, and in monitoring the natural history or therapeutic interventions of many brain disorders. Research in eye movements in pediatrics is challenging. Developing an ocular motor laboratory in children can contribute to the understanding of the mechanisms involved in brain development and plasticity in health and disease.

Methods: An infrared video-based eye tracker system was modified for studying eye movements including saccades, saccadic adaptation (an experimentally-induced dysmetria to study cerebellar plasticity), smooth pursuit, and the vestibulo-ocular reflex in seventy participants, aged 8-19 years. Thirty participants had spina bifida and Arnold-Chiari type 2 malformation (ACII) while the rest were controls. Cases from each group are illustrated.

Results: The eye tracker was found to be more sensitive than clinical examination, noninvasive, well tolerated, and acceptable to the participants and their parents. Measurements of eye movements have shown that despite ACII, smooth pursuit and the vestibuloocular reflex can be normal. ACII, however, can cause hypermetric saccades, and impair saccadic adaptation.

Conclusions: Establishing an ocular motor laboratory in pediatrics is feasible and provides a novel way to study brain function in children.

\section{General Neurology I}

\section{D-01}

Neuro-tuberculosis in Canada (1970-2000): epidemiological and clinical aspects

$M$ Phypers*, (Ottawa, ON); C Powers, (Calgary, AB)

Background: Central nervous system infection by M. tuberculosis can result in severe and often fatal neurological disease although the extent to which this occurs in Canada remains uncertain. 
Methods: Using a prospectively developed database that contains all cases of tuberculosis (TB) reported in Canada (1970-2000), we determined the incidence, demographic risk factors, clinical features and outcomes of neuro-tuberculosis (NTB), including meningitis and tuberculoma, compared to pulmonary cases.

Results: From 81,057 cases of reported tuberculosis, one percent were identified with NTB (NTB and other diagnostic sites, 810 cases; NTB exclusively, 572 cases). The incidence of NTB ranged from 14 to 39 persons per annum with a decreasing trend over time. The male:female ratio was 1.2:1. Individuals reported with NTB were younger than pulmonary cases (mean 39 SD 21, mean 50 SD 22 respectively). Analysis of NTB cases by origin revealed that 39 percent were Canadian born non-Aboriginal, 22 percent were Canadian-born Aboriginal and 39 percent foreign-born compared to pulmonary cases (49 percent, 14 percent, 36 percent respectively). Drug resistance was infrequent and similar to the pulmonary group (RR 1) whereas the likelihood of being a relapsed case was less (RR 0.4). Neuro TB had a greatly elevated risk of case fatality (RR 5).

Conclusions: NTB continues to be a serious, neurological disease and is proportionally over-represented among younger individuals and the Canadian-born Aboriginal and foreign-born populations.

\section{D-02}

Adult Alexander's disease with autosomal dominant transmission: a distinct entity caused by mutation in the GFAP gene

E Stumpf*, H Masson, F Berthelet, B Brais, P Cossette, (Montreal, $Q C)$

Background: Alexander's disease is caused by mutations in the GFAP gene. The adult form of the disease has been rarely described and its etiology remains unknown. We sought to characterize the clinical phenotype and identify the gene causing an autosomal dominant form of adult Alexander's disease.

Methods: We studied a large French-Canadian kindred. A neurological examination was performed on affected individuals. Most patients underwent a brain MRI and polysomnographic study. The diagnosis was confirmed pathologically in two individuals. We screened coding regions of the GFAPgene by direct sequencing and single stranded conformational polymorphisms analysis.

Results: We found a novel D78E mutation in GFAP in affected individuals. This mutation was not detected in 100 controls. Clinical and radiological features were different from the infantile and juvenile forms of the disease. Bulbar signs, sleep disturbance (sleep apnea), dysautonomia and dysmorphism were found in all affected individuals. In younger patients, MRI revealed T2 signal abnormalities in the medulla compatible with demyelination. Older patients had marked atrophy of the medulla without signal abnormalities.

Conclusions: This is the first demonstration of a mutation in GFAP causing an autosomal dominant form of Alexander's disease and establishes the existence of the adult variant of the disease.
D-03

Neurological manifestations of Behcet's disease: a report of 18
cases

A Borhani-Haghighi*, AR Nikseresht, SH Samangooei, N Ashjazadeh, GH Yousefipour, AH Shariat, (Shiraz, Iran)

Background: Behcet's disease is a multisystem vasculitis of unknown origin. Its neurological manifestations (Neuro-Behcet's disease) include parenchymal and nonparenchymal central nervous system (CNS) and less frequently peripheral nervous (PNS) system presentations

Methods: This retrospective study was done in the Behcet's research clinic in Shiraz (south-west Iran). Clinical records, neuroimagings, CSF analyses and electrodiagnostic studies of patients were reviewed.

Results: 18 out of 694 Behcet's patients (2.6\%) were found to have definite neurologic involvement (15 males and three females with a mean age of 34.7). All of them fulfill criteria of the International Study Group of Behcet's Disease. Headache, weakness, tingling and numbness were the most common symptoms. Corticospinal and somatosensory findings were the most frequent signs. CNS involvement was much more common than PNS manifestations. Hemispheral and brainstem syndromes and cerebral venous thrombosis were the major neurologic presentations. We had also cases with meningoencephalitic, myelitic, amyotrophic lateral sclerosis-like, multiple sclerosis-like and Guillain Barré syndrome-like presentations.

Conclusions: Neuro-Behcet's disease must be considered in the differential diagnosis of stroke in young adults, chronic meningitis, intracranial hypertension, multiple sclerosis, myelopathies and peripheral neuropathies.

\section{D-04}

\section{Clinical and neuroimaging features of HELLPsyndrome}

$J$ Chu*, (Toronto, ON)

Background: HELLP syndrome is an uncommon form of toxemia during pregnancy characterized by hemolysis, elevated liver enzymes and low platelets. Neurological presentation may include hypertensive encephalopathy, seizures, intracranial hemorrhage and ischemic infarctions.

Methods: Case report with literature review.

Results: A 39-year-old primigravida, 22 weeks twin pregnancy, presented with seizures and confusion. Examination showed her to be hypertensive $\mathrm{BP}=200 / 100$ and $\mathrm{GCS}=9$ with evidence of raised intracranial pressures and bilateral extensor plantar responses. Her gadolinium enhanced MRI of brain showed extensive cerebral edematous changes in the cerebrum,cerebellum and brain stem. CSF analysis showed no sign of infection but with elevated opening pressure and raised protein. She had a hemolytic anemia $(\mathrm{Hb} .=98$ $\mathrm{g} \%$ ) with platelet count reduced at 74,000. Her liver function tests (SGOT, SGPT, Alk. Phosphatase) were also severely elevated. She was treated with IV labetolol, MgSO4, dexamethasone, mannitol and phenytoin. Induced abortion was carried out two days after her admission. Her neurological status improved gradually with concomitant normalization of her hematological and hepatic parameters. Follow-up MRI scan of her brain two weeks later showed complete resolution of the previous abnormalities. She was

Suppl. $2-$ S 18 
seen one month following discharge with entirely normal neurological examination and BP.

Conclusions: Prompt and early recognition of HELLPsyndrome would be important to prevent permanent neurological sequelae. The pathophysiology of cerebral edema in HELLP syndrome was thought to be due to vasospasm and vascular endothelial cell dysfunction causing disruption of the blood-brain barrier. A Medline search indicates many case reports showing complete resolution of MRI changes in follow-up coinciding with neurological recovery.

\section{D-05}

The Vancouver experience following an outbreak of heroininduced leukoencephalopathy

$S$ Gul $^{*}$, S Spacey, (Vancouver, BC)

Background: The inhalation of heated heroin vapour (heroin pyrolysate) is a growing practice amongst drug users who wish to avoid the risks associated with the parenteral administration of this drug. This practice, also known as "chasing the dragon", has been described through scattered case reports in the literature, to result in a progressive spongiform leukoencephalopathy.

Methods: Between January and March of 2002, four cases of heroin-induced leukoencephalopathy were diagnosed at the Vancouver General Hospital. We followed these patients clinically and obtained serial MR imaging. MR spectroscopy was also obtained. Two patients died as a result of their disease and postmortem brain examinations were performed. Follow-up assessments were available to 10 months postadmission in the two survivors.

Results: The four patients in our study presented with varying degrees of lethargy, dysarthria and bradykinesia. In our second patient, there was also marked cerebellar dysfunction. In each case, the patient's symptomatology progressed over a period of two to seven weeks prior to admission to hospital. CT scans consistently demonstrated symmetric, white matter low density changes. MR scans in all four patients revealed extensive white matter high signal change on T2 and FLAIR sequences. Post-mortem examinations demonstrated widespread white matter edema with relative sparing of the U-fibers. Microscopy revealed vacuolar degeneration with axonal sparing.

Conclusions: Despite a considerable population of heroin users, these four cases represented the first confirmed outbreak of heroininduced leukoencephalopathy in the Greater Vancouver area.

\section{D-06}

Pallidal deep brain stimulation and L-dopa do not improve qualitative aspects of skilled reaching in Parkinson's disease

T Hansen, Z Kiss, O Suchowersky*, (Calgary, AB); J Doan, L Brown, I Whishaw, S Pellis, (Lethbridge, AB)

Objective: To determine the effects of dopaminergic medication and pallidal deep brain stimulation (DBS) on the quality of skilled reach in patients with Parkinson's disease (PD).

Background: Parkinson's disease is a neurodegenerative disorder affecting motor control. Symptoms are currently treated with L-dopa and DBS. While speed and execution of movements are improved by L-dopa, not all of the motor symptoms are alleviated. Little is known about the effects of DBS or medication on the parameters of skilled reaching.
Design method: Nine male and two female PD patients (mean age $=62$ yrs) with unilateral pallidal DBS reached with the contralateral hand for a small piece of food, placing it in the mouth, and return the arm to the starting position. Testing was performed independently in four conditions: no treatment, medication only, DSB only, and combined treatment. Patients were compared to 14 age-matched healthy controls. Reaches were digitally recorded and analyzed on a 21 point scale adapted from Eshkol-Wachman Movement Notation. The 21 point scale was reduced to seven subsets that included orientation, lift, aim, pronation, grasp, supination, and return. Trained raters were blinded to condition and side of DBS. All trials were randomized.

Results: Patients were tested 6-36 months after surgery. All showed significant improvement clinically and in UPDRS scores with treatment. The following data were obtained on the reaching scale (maximum of 21 points): normal controls $16.5-21.0$ (mean 18.3), patients with no treatment $3.0-14.0$ (mean 9.2), medication only $7.0-14.0$ (mean 10.3), DBS only $4.5-16.0$ (mean 10.3), and combined treatment $4.0-16.5$ (mean 10.5). The difference between controls and all treatment groups was statistically significant $(\mathrm{p}$ $<.001$ ). Patients scored significantly lower in each of the seven subsets relative to controls suggesting that all aspects of reach were compromised. No significant differences were found among the four patient conditions.

Conclusions: The results of this study are consistent with accumulating evidence indicating that some aspects of motor performance in PD patients, in this case reaching and grasping, are resistant to L-dopa. In addition, pallidal DBS does not improve parameters resistant to L-dopa, either in combination with medication or alone.

\section{D-07}

Mutations in the epsilon sarcoglycan gene do not account for most cases of the myoclonus-dystonia syndrome

D Grimes*, F Han, D Bulman, (Ottawa, ON); A Lang, (Toronto, $O N)$

Background: Myoclonus-dystonia syndrome (MDS) (OMIM \#159900) is typically an autosomal dominant disorder with reduced penetrance and variable expression. It is characterized by alcoholsensitive nonprogressive myoclonic jerks involving mainly the arms and axial muscles often seen in combination with dystonia. Mutations in the epsilon sarcoglycan (SGCE) gene on chromosome $7 \mathrm{q} 21$ are emerging as the major causative factor for this condition.

Methods: Genomic DNAwas extracted from the venous blood of patients and two microlitres $(100 \mathrm{ng} / \mathrm{ml})$ of DNA was used to amplify each of the 11 exons of the SGCE gene in 22 affected individuals from seven unrelated families.

Results: We detected mutations in the SGCE gene in only three of the seven families screened. The proband from family 4 was found to carry a heterozygous 5-basepair deletion (5'-AAAAC-3') in exon 7 of the SGCE gene. This deletion leads to a frameshift of the coding region with a premature stop at codon 294 . This deletion was not identified in 200 chromosomes screened. Two families carry nonsense mutations in exon 3 , R102X (304C to T) that have been identified previously.

Conclusions: Although mutations in the SGCE gene appear to be a common cause for the typical phenotype of MDS they do not account for all cases. 


\section{D-08}

The pendular nystagmus of oculopalatal tremor: imaging correlations

James A Sharpe*, Ji Soo Kim, (Toronto, ON)

Background: Patterns of nystagmus in oculopalatal tremor (OPT) is associated with hypertrophy of the inferior olivary nucleus (ION). Mixed torsional-vertical pendular nystagmus in OPT has been considered to signify unilateral brainstem damage and symmetrical vertical nystagmus considered to indicate bilateral disease. We assessed these concepts by oculography and imaging.

Methods: Ocular oscillations were analyzed in 10 patients, using a magnetic search coil technique in nine patients and video recording in one. MRI was performed in eight patients.

Results: Six patients had conjugate vertical pendular nystagmus and four showed mixed torsional-vertical pendular nystagmus. Two of them also had vergence pendular oscillation. MRI demonstrated increased signal or hypertrophy of the ION, unilateral in five and bilateral in three. Unilateral olivary signal changes were associated with conjugate vertical pendular nystagmus in two patients, and with mixed torsional-vertical nystagmus in three patients. Bilateral olivary changes were visible in two patients with conjugate vertical pendular nystagmus and in one patient with mixed torsional-vertical nystagmus. Palatal tremor appeared to be bilateral in all patients. OPT resolved in one patient, after 20 years, despite persistence of signal change in the ION.

Conclusions: Brain imaging indicates that conjugate vertical and mixed torsional-vertical pendular nystagmus do not correspond to the laterality of signal changes in the ION. Either nystagmus form may occur with unilateral or bilateral signal changes in the ION. OPT may rarely subside spontaneously.

Supported by an Elizabeth Barford Award, University of Toronto (JSK), and by Canadian Institutes of Health Research (CIHR) grants ME 5509 and MT15362

\section{SPINE}

\section{E-01}

Novel synthetic grafts promote axonal regeneration and functional recovery after spinal cord transection

E Tsai*, R DeMarchi, P Dalton, M Shoichet, C Tator, (Toronto, $O N)$

Co-winner of the Canadian Neurosurgical Society's K.G. McKenzie Prize in Basic Neuroscience Research (see page 11)

\section{E-02}

\section{Intraoperative EMG and SSEP recordings in a prospective study of 213 patients undergoing spinal surgery: correlation of electrophysiological changes and postoperative neurological deficits}

T Gunnarsson*, A Krassioukov, R Sarjeant, M Fehlings, (Toronto, $O N)$

Background: Continuous intra-operative electromyography (EMG) and somatosensory evoked potentials (SSEP) are frequently used in spinal surgery to prevent or predict neural injury. However, only limited data are available on the sensitivity and predictive value of intra-operative electrophysiological changes with regard to the occurrence of postoperative neurological deficits.

Methods: We examined data prospectively on 213 patients with various spinal diseases who underwent intra-operative monitoring with EMG and SSEP. Complete clinical neurological follow-up and electrophysiological data were available in all cases. The present analysis focused on the correlation of intra-operative electrophysiological changes with the development of new neurological deficits.

Results: Fifteen patients developed new neurological deficits. Eleven patients had new sensory changes, 10 had new motor deficits (seven new leg muscle weakness, three bladder dysfunction) and six had combined motor and sensory deficits. Significant changes in SSEPwere detected in four cases of which three developed sensory symptoms and one motor weakness. Significant activation of intraoperative EMG was detected in nine of 10 patients with new motor deficits.

Conclusions: Monitoring with combined SSEPs and real-time EMGs significantly enhances the sensitivity of detection of neurophysiological changes particularly in patients undergoing thoracolumbar procedures. New postoperative neurological deficits generally correlate well with intraoperative neurophysiological findings.

\section{E-03}

\section{Anterior cervical plating: a survey of Canadian practice patterns}

GE Pickett*, J Van Soelen, N Duggal, (London, ON)

Background: The need for anterior cervical instrumentation and the optimum type of plate for patients undergoing anterior cervical discectomy and fusion (ACDF) for single-level degenerative disease is controversial. We surveyed Canadian spinal surgeons to ascertain current practice patterns and possible demographic influences.

Methods: We conducted a web-based survey of neurosurgeons and spinal orthopedic surgeons in Canada. Questions asked included preferred fusion techniques, frequency of use and favoured type of anterior cervical instrumentation, indications for plating, and the use of external cervical orthoses following ACDF with or without plating.

Results: Of 60 eligible respondents, $72 \%$ were in academic practice, and 59\% were neurosurgeons. Neurosurgeons employed plates in $42 \%$ of ACDF cases, whereas orthopedic surgeons used them in $70 \%$ of cases. Constrained and semi-constrained plates were chosen with equal frequency. Cervical orthoses were recommended for $61 \%$ of patients with plates, and $92 \%$ of patients without plates. Surgeons in practice for less than five years were more likely to plate and to recommend collars.

Conclusions: The use of anterior cervical plating in ACDF for single-level degenerative disease is influenced by training background, type and length of practice. Plating reduces but does not eliminate the use of postoperative external cervical immobilization. 
E-04

Gender-related differences regarding secondary complications in individuals with spinal cord injury: assessment of a consecutive series of $\mathbf{5 8}$ cases

\section{J Furlan*, A Krassioukov, M Fehlings, (Toronto, ON)}

Background: Little is known regarding the influence of gender on secondary complications in individuals with acute traumatic spinal cord injury (SCI).

Methods: All spinal cord injured individuals admitted to an acute care unit at the University of Toronto from 1998-2000 were included in this retrospective analysis. Data were analyzed using Fisher's Exact, Chi-square, Student-t tests.

Results: There were 58 SCI individuals (15F, 43M, ages $17-89$ years, mean of 55.4). Female and male groups were similar regarding age (means of 62.9 and 52.8 years, respectively; $\mathrm{p}=0.097$ ), medical co-morbidities ( $25.5 \%$ versus $27.3 \% ; \mathrm{p}=1)$, as well as cause $(p=0.403)$, level $(p=0.664)$, and severity of SCI $(p=0.484)$. There were no differences between women and men regarding length-ofstay (43.4 \pm 16.7 versus $47.8 \pm 20.5$ days; $\mathrm{p}=0.749)$, mortality rate $(13.3 \%, 9.3 \% ; \mathrm{p}=0.643)$, and secondary complications $(46.7 \%$, $44.2 \% ; \mathrm{p}=1)$. The most common secondary complications in women and men were infection $(40 \%, 32.6 \% ; \mathrm{p}=0.754)$, psychiatric disorder (26.7\%, 11.6\%; $\mathrm{p}=0.218)$, pressure sore $(13.3 \%, 13.9 \% ; \mathrm{p}=1)$, cardiovascular complications $(6.7 \%, 13.9 \% ; \mathrm{p}=0.664)$, clotting disorder $(13.3 \%, 7 \% ; \mathrm{p}=0.596)$, and autonomic dysreflexia $(6.7 \%$, $7 \% ; \mathrm{p}=1)$.

Conclusions: Although SCI is a devastating event that occurs more frequently in men than women (3:1), no significant genderrelated differences were observed regarding demographics and clinical outcomes including secondary complications during acute stage after SCI.

\section{E-05}

Surgical treatment of cervical myeloradiculopathy associated with movement disorders: indications, techniques and outcomes in a consecutive, prospective series

A Wong*, E Massicotte, M Fehlings, (Toronto, ON)

Background: Movement disorders may be associated with advanced cervical myeloradiculopathy and present unique diagnostic and management challenges.

Methods: We report on eight patients with movement disorders causing cervical myeloradiculopathy. The demographics, clinical parameters, duration from the diagnosis to the onset of the symptoms and from onset of symptoms to operation, levels of operation and types of operations performed were summarized. Our management strategy and results were compared with a systematic review of the literature.

Results: The mean age of our patients was 44 years with a M:F ratio of 6:2. The average duration of onset of symptom was 10 months. We had three cases of cerebral palsy, two cases each of dystonia and Tourette syndrome and a case of torticollis post spinal-injury. The most common levels decompressed were C3-4 and C4-5. Three cases also had botulinum toxin injections. Six cases showed improvement and two cases showed stabilization of neurological status. The literature review showed a total of 78 patients with an average age of 43 years and an average duration of 2.3 years prior to presentation.
Conclusions: Movement disorders cause premature cervical spondylosis most commonly involving the C3-4 and C4-5 levels. Perioperative use of halo vest and botulinum toxin injection is recommended in selected cases to control cervical movements. Combined decompression and rigid internal fixation is strongly recommended. Laminectomy with lateral mass fixation may be used successfully in the absence of kyphotic deformity.

\section{E-06}

Assessment of novel neuroprotective therapies for acute spinal cord injury in the "postmethylprednisolone era": role of sodium/glutamate blockers

G Schwartz*, Y Liu, M Fehlings, (Toronto, ON)

Background: Methylprednisolone (MPS) remains the only pharmacotherapeutic agent used clinically after spinal cord injury (SCI). Given the modest benefits and potential toxicity of MPS, other neuroprotective approaches are required.

Methods: Previously, we showed that acute administration of riluzole (Ril), a sodium channel blocker, is neuroprotective in rodents after SCI. Further experimentation to determine Ril's and the novel sodium channel blocker topiramate's (TP) optimal dose, time window for administration, and potential synergy with MPS was done in an in vitro organotypic slice model of SCI. Weight-drop injured slices were treated at several time points pre and post SCI with $10 \mu \mathrm{M}$ of Ril, TP, MPS, or Ril+MPS. Fluorescent, propidium iodide (PI) -labeled cells were imaged by confocal microscopy at designated times post treatment.

Results: Mean PI cell counts from each treatment group were quantified to generate an index of cell death. Analysis by ANOVA indicated a significant effect of treatment $\left(\mathrm{p}=3.7 \times 10^{-9}\right.$ and $\mathrm{p}=9.29 \times 10^{-13}$ ) with the neuroprotective drug treated slices consistently having less cell death than control slices.

Conclusions: These findings suggest that the sodium channel blockers, Ril and TP, and MPS are effective neuroprotective strategies for SCI. Further preclinical in vivo evaluation is warranted prior to translation to clinical trials.

\section{STROKE}

\section{F-01}

Transcranial doppler IMS criteria for rescued intra-arterial thrombolysis: multicenter experience from the interventional management study

Maher Saqqur*, Ashfaq Shuaib, (Edmonton, AB); Andrei Alexandrov, Sergio Calleja, (Houston, TX); Michael Hill, Andrew Demchuk, (Calgary, $A B$ )

Background: Combined therapy using intravenous (IV) tissue plasminogen activator (tPA) and intra-arterial (IA) tPA has potential to enhance recanalization rates and improve outcome when compared to IVtPA alone. Transcranial Doppler (TCD) has potential to screen which IV tPA patients have persistent occlusion and require further IAintervention. We examined the clinical utility and prognostic value of TCD flow findings in patients enrolled in the Interventional Management Study (IMS) IV/IAtPA pilot trial.

Methods: Patients enrolled in the IMS trial who underwent 
urgent TCD performed prior to IV tPA bolus or IA tPA were included. TCD findings were analysed to predict outcome and identify which findings correctly predicted an arterial occlusion proximal enough that IA tPA was attempted (internal carotid artery (ICA), M1 or M2 middle cerebral artery (MCA)).

Results: 29 of 80 patients enrolled in the IMS trial had pre-IA lysis TCD (mean age 62.6, men: women 17:12, baseline median NIHSS 17). No temporal windows were found in three patients $(10 \%)$. The affected MCA mean flow velocity (MFV) / unaffected MCAMFVratio less than 0.6 had sensitivity $89 \%$ (95\%CI 65-99\%); specificity $100 \%(54 \%-100 \%)$ 97.5\% one-sided CI; PPV $100 \%$ $(79 \%-100 \%) * 97.5 \%$ one-sided CI; NPV 75\% (95\%CI 35\%-97\%) for identifying patients with IA lytic requiring occlusion in the anterior circulation. (Mean ratio IA lysis lesion present vs absent; 0.32 vs. 1.09 , $\mathrm{p}$ value less than 0.001) Clinical outcome correlated with mean MCA MFV (patients with three month Rankin of 3-6: 8 $\mathrm{cm} / \mathrm{sec}$ vs. Rankin 0-2: $32 \mathrm{~cm} / \mathrm{sec} ; \mathrm{p}=0.003$ ) All patients with absent MCAflow signals had poor outcomes (mRS more than two at three months) despite treatment in the IMS protocol ( $\mathrm{p}=0.003)$.

Conclusions: TCD has significant potential as a screening tool for IV/IA lysis protocols. The ratio of affected MCA MFV/unaffected MCA MFV less than 0.6 appears to be sensitive and specific for identifying arterial occlusions that require additional intra-arterial intervention. Absent MCAflow is associated with poor outcome despite treatment.

\section{F-02}

Site of arterial occlusion identified by transcranial Doppler (TCD) predicts response to intravenous stroke thrombolysis

Maher Saqqur*, Ashfaq Shuaib, (Edmonton, AB); Andrei Alexandrov, Ken Uchina, (Houston, TX); Carlos Molina,

(Barcelona, Spain); Andrew Demchuk, (Calgary, AB)

Background: Intravenous (IV) tissue plasminogen activator (tPA) remains the only treatment approved in North America for acute ischemic stroke. Angiographic studies with intra-arterial thrombolysis suggest that more proximal occlusions carry greater thrombus burden and are less likely to benefit from local therapy. Little data is known regarding whether IV tPA has similar treatment differences depending on site of occlusion.

Design/Methods: We used established specific TCD criteria to determine the location of arterial occlusion. Occlusion was classified into the following locations: distal MCA, proximal MCA, cervical ICA plus MCA, terminal ICA, or basilar artery. We compared the rate of good outcome at three months (modified Rankin Scale $(\mathrm{mRS}))$ and dramatic recovery (NIHSS less than 2 at 24 hours) for each occlusion group. We used NIHSS less than 2 at 24 hours as the early measure of response to thrombolysis based on NINDS tPA trial data. (Broderick et al, Stroke 2000).

Results: 222 patients had TCD prior to or during IV tPA treatment. Mean age 64.3, male 113: female 109. Median baseline NIHSS score 17. Median time to TCD examination 130 minutes from symptom onset. Median time to IV tPA 135 minutes from symptom onset. Median CT ASPECTS score 9. Modified Rankin Scale less than 1 was achieved in $35 \%$ of all patients. By each site of occlusion the percentage of NIHSS less than 2 at 24 hours was: distal MCA occlusion 25\% ( $\mathrm{n}=98)$; proximal MCA occlusion $15 \%$ $(n=82)$; tandem cervical ICA and MCA occlusion 36\% $(n=14)$; terminal ICAocclusion $0 \%(\mathrm{n}=19)$; and basilar occlusion $40 \%(\mathrm{n}=5)$ $(\mathrm{p}=0.011)$. By each site of occlusion the percentage of $\mathrm{mRS}$ less than 1 was: distal MCA occlusion $40.7 \%(\mathrm{n}=81)$; proximal MCA occlusion $27.3 \%(\mathrm{n}=66)$; tandem cervical ICA and MCA occlusion $30 \%(n=14)$; terminal ICA occlusion $14.3 \% \quad(n=19)$; basilar occlusion $30 \%(\mathrm{n}=5) \quad(\mathrm{p}=\mathrm{NS})$.

Conclusions: Early response to thrombolysis is influenced by the site of occlusion. Terminal ICAocclusions are least likely to respond early or long term. A significant proportion of patients with any occlusion site including basilar occlusion and tandem ICAand MCA occlusion achieve good outcome with IVtPA alone.

\section{F-03}

Plaque morphology and serum markers of inflammation in carotid stenosis: a predictive model

C Voll*, T Lewis, D Fladeland, T Ross, J Lawson, (Saskatoon, SK)

Objective: To develop a predictive model of symptomatic carotid stenosis using carotid plaque morphology combined with serum markers of inflammation.

Methods: Twenty-seven subjects with symptomatic and thirtyfive subjects with asymptomatic carotid stenosis $>40 \%$ on carotid ultrasonography were prospectively consented and enrolled. Creactive protein (CRP), Chlamydia pneumoniae serology and peripheral white blood cell count (WBC) were evaluated along with traditional cerebrovascular risk factors at baseline. Carotid plaque morphology was evaluated by B-mode ultrasonography. Logistic regression methods were used to develop a predictive model for symptomatic carotid stenosis.

Results: For each $1.0 \times 10^{9} / \mathrm{L}$ increase in WBC count, the odds ratio of symptomatic disease was 1.3 (95\% CI, 1.004 to 1.719; $\mathrm{p}=0.047$ ). Elevation of the CRP level was associated with an odds ratio of 5.3 (95\% CI, 1.63 to 27.19; $\mathrm{p}=0.005$ ) and homogeneous plaque with an odds ratio 3.1 (95\% CI, 10.091 to $1.148 ; \mathrm{p}=0.081)$ for symptomatic disease in bivariate analysis. Multivariate logistic regression analysis confirmed significant associations for CRP and homogeneous plaque in the final model.

Conclusions: The degree of elevation of WBC count, elevation of the CRP>4 and homogeneous plaque on carotid ultrasonography are significantly and independently associated with symptomatic carotid stenosis.

\section{F-04}

The reliability and pitfalls of B-mode ultrasound standardized values for measurement of carotid bifurcation stenoses compared to MR angiogram and digital subtraction angiogram

Farhad Pirouzmand, Venkat Sadanand, Colin Honish*, (Saskatoon, $S K)$

Background: Carotid ultrasounds (US) are routinely performed as screening tests on patients who present with TIAs or strokes. Many of these patients then undergo DSAor MRA. DSAis invasive with its inherent risks and financial costs. MRA is an evolving technology with unsubstantiated reliability. We perform a retrospective analysis comparing the reliability of MRA and US modes with the gold standard DSA in determining the degree of stenosis.

Methods: Five year (1998-2002) retrospective analysis of 
patients who had carotid DSAand who had prior US. Some of them had MRA as well. We collected data on epidemiology, plaque characteristics and NASCET degree of stenosis on 140 carotid arteries. The measured US parameters were peak systolic velocity (PSV), end diastolic velocity (EDV), ICA/CCA velocity ratio (VR) and plaque characteristics. MRA parameter was degree of stenosis. The analysis included ROC curves and Kappa measurement with SPSS10 package.

Results: On angiograms, $34 \%$ of the patients had high grade stenosis $(>70 \%)$ and $18 \%$ had moderate grade stenosis $(50 \%-69 \%)$. Using ROC curves the best combination of sensitivity and specificity was seen for PSV $>173$ as follows: sensitivity 0.87 , specificity 0.80 , PPV 0.70 and NPV 0.93 . With our traditional PSV of $>230$ as the cutoff point, the sensitivity was $69 \%$ with specificity $90 \%$. The weighted kappa was $71 \%$ and $62 \%$ respectively for these two PSV. The 19 MRAs had a kappa correlation with DSA of 0.78 (sensitivity $75 \%$, specificity $100 \%$, PPV 1.0 , NPV 0.85 ).

Conclusions: The US values in each center used for prediction of carotid stenosis should be validated. In our center PSV $>173$ is the best predictor of significant carotid stenosis. MRA has a perfect specificity and PPV but this technique needs to be standardized and improved. Simultaneous use of MRA and US as the screening test increases the overall sensitivity to over $90 \%$ without compromising the specificity.

\section{F-05}

The relationship between supra-annual trends in influenza rates and stroke occurrence

MD Hill*, TS Field, M Tarrant, H Zhu, JR Mitchell, (Calgary, AB)

Introduction: An association between acute and chronic infections and increased risk of ischemic stroke has been reported. An association between influenza vaccination rates and reduced risk of brain infarction has been reported. We sought to determine the relationship between population-based influenza activity and stroke occurrence.

Methods: Administrative data from an eight-year period beginning January 1, 1994 and ending December 31, 2001, were examined from the Calgary Health Region (pop. 1.4 million). All patients with a discharge diagnosis code (ICD9) of 430, 431, 432, $433,434,435$ or 436 in the first diagnosis position were included. Alberta ViralWatch is a government-funded program that compiles data on influenza rates. The study was approved by the Calgary Health Region Ethics Board.

The population of the Calgary Health Region for each week of the year was calculated based upon yearly national data available from Statistics Canada, using a natural logarithmic function. The rates of influenza and other respiratory illnesses were then calculated using this population estimate. To assess the baseline trends in stroke rates and influenza rates, the frequency components of both the influenza and stroke data were isolated using Fourier transformation. Linear regression corrected for serial autocorrelation of residuals (Prais-Winsten regression) was used to assess the relationship between transformed influenza and stroke rates.

Results: Prais-Winsten linear regression demonstrated a relationship between supra-annual (4-term Fourier transform) stroke occurrence rates and influenza-like illness rates. The relationship showed a positive slope meaning that as influenza rates increased, stroke rates increased. Within the range of influenza-like illness, a tripling of the influenza-like illness rate, was only associated with an approximate $6 \%$ increase in stroke occurrence rate.

Conclusion: Variation in population influenza rates is associated with a small proportion of variance in population stroke rates. The effect is substantial on a population wide basis.

\section{F-06}

Stent-assisted angioplasty of intracranial vertebrobasilar atherosclerosis: clinical and imaging mid-term results

P Rasmussen, M Chow*, H Woo, M Mayberg, T Masaryk, (Cleveland, OH); J Perl, (Minneapolis, MN)

Background: Initial reports of treatment with stent-assisted angioplasty suggest this is a feasible treatment, but no data exist on long-term follow-up. We report our mid-term results and experience with 39 patients with medically refractory TIAs secondary to intracranial posterior circulation atherosclerotic occlusive lesions treated with stent-assisted angioplasty.

Methods: Thirty-nine patients (35 male, ages 43 - 80 years) experiencing signs and symptoms of vertebrobasilar insufficiency refractory to coumadin and/or anti-platelet agents underwent 40 stent-assisted angioplasty procedures. Angiography revealed severe intra-cranial vertebral $(\mathrm{n}=20)$, basilar $(\mathrm{n}=18)$, or basilar and vertebral $(n=2)$ stenoses. Patients were evaluated prior to treatment and in follow-up with transcranial Doppler ultrasound (TCD).

Results: Thirty-eight of 39 patients were successfully revascularized with $0-38 \%($ mean $=10.0 \%)$ residual stenosis. Two patients $(5 \%)$ died in the peri-procedure period. Seven patients (18\%) had neurologic complications, two patients $(5 \%)$ had transient neurologic symptoms. Thirty-two of 35 surviving patients $(91 \%)$ were asymptomatic with a median follow-up of 6 months (range 036 months). There have been no documented re-stenoses or stent occlusions. No statistically significant predictors of neurological morbidity and mortality were discovered, however, there was a trend found with basilar lesions, tandem lesions and those patients with contralateral vertebral occlusions.

Conclusions: Although primary intracranial vertebrobasilar artery stenting is associated with complications that can be life threatening, when successful, the procedure is highly efficacious in symptom improvement. Moreover, the results appear to be durable up to 24 months.

\section{NeUROMUSCULAR}

\section{G-01}

Gene therapy for myotonic dystrophy by preferential targeting of mutant myotonin protein kinase mRNA

\section{M-A Langlois*, G Doucet, J Puymirat, (Quebec, QC); JJ Rossi, (Los Angeles, California)}

Background: Myotonic dystrophy type 1 (DM1) is an autosomal dominant neuromuscular disease characterized by myotonia, skeletal muscle weakness and wasting, cardiac conduction problems, cataracts and other multi-system dysfunctions. The molecular defect associated with DM1 is a large CTG expansion in 
3'UTR of the DM protein kinase (DMPK) gene. Evidence now shows that specific targeting of mutant DMPK transcripts may prove successful in alleviating many of the cellular and molecular phenotypes seen in DM1. Until now, no method has proven successful in targeting preferentially the mutant transcripts. Here we have devised two approaches for targeting mutant DMPK mRNAs, one using antisense RNAs the other using nuclear-retained ribozymes.

Methods: We have designed a retroviral system capable of expressing high levels of an antisense RNA directed to a site in 3'UTR of the DMPK mRNA encompassing the CUG repeats. We also developed a ribozyme expressed under the control of a modified tRNA-meti promoter. The modifications in this expression cassette causes nuclear retention of its expression products. Accessible ribozyme cleavage sites were identified within the 3'UTR of DMPK mRNA by semi-random oligonucleotide scanning and a hammerhead ribozyme was designed to cut the most accessible sequence tested.

Results: In vitro assays show that the antisense RNA produced reduces levels of mutant and normal DMPK mRNA by $50 \%$ and $80 \%$ respectively. This effect is also associated with restoration of glucose uptake, myoblast differentiation and reduction of CUGBP1 sequestration in the nucleus. Transient transfection of DM1 myoblasts with the wild type ribozyme expression cassette shows a $62 \%$ and a $50 \%$ reduction in mutant and normal transcript expression. In-situ hybridization using a Cy3-labeled DMPK probe showed a significant reduction in the number of nuclear speckles in ribozyme-transfected myoblasts. Destruction of mutant DMPK mRNA by the wild type ribozyme also coincided with insulin receptor splicing restoration by nearly $32 \%$.

Conclusions: Our results show for the first time that molecular targeting of the nuclear-retained mutant DMPK mRNAby antisense RNAs and ribozymes is effective in restoring many of the cellular and molecular phenotypes associated with DM1. Thus, these strategies offer a promise of possible therapeutics for the near future, once an effective and safe delivery system is developed.

\section{G-02}

\section{Improvement of grafted myoblast survival within mdx muscles by tri-substituted purines}

\section{E El Fahime*, M Bouchentouf, B Benabdellah, P Mills, JP Lafrenièrre, J-P Temblay, (Quebec, QC)}

Background: Myoblast transplantation is developing into a therapeutic approach in Duchenne's dystrophy's disease. A major limiting factor is that only a small number of grafted myoblasts survive the procedure.

A 2,6,9-tri-substituted purine was shown to affect the expression of a variety of growth factors, immunomodulatory, extracellular matrix-remodeling and stress response genes. Since the effects of these purines are consistent with the activation of pathways involved in wound healing and tissue regeneration, we have investigated whether pretreatment of myoblasts with a new tri-substituted purine (tubulysin) could reduce myoblast death after transplantation.

Methods: In vitro cell death was assessed by annexin-Vlabeling, while in vivo the disappearance of transplanted myoblast within mdx mice muscle was evaluated by the loss of radio-labeled thymidine of donor DNA.
Results: In vitro, we show that tubulysin prevents myoblasts from apoptosis induced by staurosporine. In vivo, the pretreatment and coinjection of normal myoblasts with tubulysin significantly increased the survival of myoblasts transplanted into mdx mice, thus giving rise to more hybrid myofibers compared to untreated cells.

Conclusions: Tubulysin can be used as an in vivo survival factor to improve the myoblast-mediated ex vivo gene transfer approach.

\section{G-03}

Diabetic autonomic neuropathy: spectrum of clinical involvement

T Townsend*, G Bartlett, J Stewart, (Montreal, QC)

Background: Unlike polyneuropathy (PN), diabetic autonomic neuropathy (DAN) has been little studied. Our objectives were to evaluate: 1. Pattern of involvement of different organs/systems in DAN; 2. Relationship of DAN to PN, and duration, type of diabetes mellitus (DM) and other microvascular complications.

Methods: Chart review of patients with DAN symptoms. Autonomic dysfunction assessed by symptoms. Organs/systems evaluated: cardiovascular (CV), bladder (BL), upper (UGI) and lower gastrointestinal tract (LGI), anorectal incontinence (ARI), lacrimation and salivation (LR), sweating (SW), and erectile dysfunction (ED) in males. Polyneuropathy quantitated.

Results: 95 patients ( 75 men). Of eight ANS organs/systems in men and seven in women (the difference being ED), mean involvement was 2.5 (SD 1.3); minimum 1, maximum 8. Frequency of involvement was: CVS 59\%, SW 34\%, LGI 33\%, BL 27\%, UGI $24 \%$, LS $10 \%$, ARI 5\%. ED present in $80 \%$ men. Mean PN score was 1.6 (of maximum 4) (SD 0.8). Slight correlation between PN severity and number of ANS organs/systems involved (r-0.21, pvalue 0.04 ). No correlation between DAN and duration, type of DM, or to retinopathy or nephropathy.

Conclusions: 1. DAN produces patchy and selective symptomatic autonomic dysfunction. 2. DAN correlates weakly with PN severity, but not with duration or type of DM, retinopathy or nephropathy.

\section{G-04}

Autosomal recessive ataxia associated with a CMT-like polyneuropathy and elevated alpha-fetoprotein

A Duquette*, J McNabb-Baltar, B Brais, L Marchand, (Montreal, QC); JP Bouchard, (Quebec, QC); J Mathieu, (Saguenay, QC)

Background: This study clinically and genetically characterizes a distinct group of French Canadians with an association of ataxia, severe peripheral neuropathy and elevated serum alpha-fetoprotein.

Methods: Patients were examined, electrophysiological data was compiled and alpha-fetoprotein levels were measured. Polymorphic markers were typed for candidate regions. Standard genotyping and linkage were performed.

Results: The 24 patients from 10 families present a homogeneous phenotype consisting of an ataxia appearing between ages two and 20 (mean 14) with a severe CMT-like polyneuropathy. In all tested cases the alpha-fetoprotein was increased (1.4 to 18.2 times normal, mean 5.5, $\mathrm{n}=16$ ). Ocular apraxia was featured in some older cases. Radiological studies showed pontocerebellar atrophy. DNA for 17 patients was haplotyped. No allele sharing was observed for the 
ataxia with ocular apraxia (AOA) and spinocerebellar ataxia with axonal neuropathy (SCAN1) loci. Linkage disequilibrium was observed in the recessive spinocerebellar ataxia non Friedreich type 1 (SCAR1) locus on chromosome 9q34. There was a shared haplotype for 13 of 18 carrier chromosomes (72\%). Four families gave a maximum LOD score of 2.35 at a theta of 0 for marker D9S1818.

Conclusions: Linkage analyses demonstrate that recessive ataxia in these French Canadian families is linked to the 9q34 SCAR1 locus.

\section{G-05}

Volumetric analysis by manual segmentation in amyotrophic lateral sclerosis

S Kalra*, (Edmonton, AB); J Jirsch, E Ehrensperger, K Shahrour,

A Genge, DL Arnold, (Montreal, QC)

Background: Imaging and pathological studies to date have reported varying degrees of brain atrophy in amyotrophic lateral sclerosis (ALS). We sought to quantitate regional volume changes in ALS using manual segmentation of MRI scans.

Methods: Thirty-eight subjects with ALS and 19 controls had a T1-weighted 3D gradient-echo brain MRI. The peri-rolandic gyri, corticospinal tract (posterior limb of the internal capsule and cerebral peduncles), and corpus callosum were manually segmented on an image processing program for volume determination.

Results: There was no difference in volumes between ALS and controls for the cerebral peduncle $\left(1.2 \pm 0.09 \mathrm{~cm}^{3}\right.$ vs. $1.1 \pm 0.1$, $\mathrm{p}=0.3)$ and the corpus callosum $(0.75 \pm 0.1$ vs. $0.80 \pm 0.1, \mathrm{p}=0.1)$. Internal capsule volume ascertainment was completed for 10 subjects in each group $(0.90 \pm 0.02$ vs. $0.87 \pm 0.02, \mathrm{p}=0.3)$. Segmentation of the peri-rolandic cortices could not be done with confidence due to their variability and lack of definable boundaries.

Conclusions: In contradiction to previous reports the corticospinal tracts and corpus callosum are not atrophied in ALS suggesting that neuronal dysfunction, in addition to neuronal loss, plays a role in contributing to disability. Due to anatomic complexities, techniques other than manual segmentation are necessary for volumetric analysis of the peri-rolandic cortex.

\section{EPILEPSY}

\section{H-01}

High field functional MRI analysis of interictal discharges in malformations of cortical development: role of surround inhibition and subcortical structures

P Federico*, J Archer, D Abbott, G Jackson, (Melbourne, Australia)

Winner of the Canadian Neurological Society Frances McNaughton Memorial Prize (see page 9).

\section{H-02}

Voluntary and emotional dissociation of lower facial movements and epilepsy: a study of photographs of monozygotic quintuplets

G Remillard*, B Zifkin, J Courville, F Andermann, (Montreal, QC)

Background: The voluntary pathway for facial movement likely originates mainly from primary motor structures. Other regions may subserve emotional facial movements. Diminished lower facial contraction was observed contralateral to a temporal lobe epileptic focus in $73 \%$ of patients, more noticeable for emotional movements. Facial asymmetry and lower facial movements predominating on the left are known in the general population, ascribed to right hemispheric predominance for left lower facial movements and emotions; and is thought related to functional cerebral asymmetry. One more commonly observes leftward deviation of the lower face, especially during emotional expression.

Methods: We studied available history of the monozygotic Dionne Quintuplets, and their photographs on the Internet at www.city.north-bay.on.ca/QUINTS/DIGITIZE/partnerf.htm.

Results: Soon after birth, the quintuplets were noted to have an identical slant of their mouths from left to right. During her teen years, probably in relation to epilepsy, Emilie Dionne developed marked diminution of left lower facial movements when smiling at the camera, while her sisters retained their apparently constitutional asymmetry with left predominance. Emilie was the only left-handed Dionne and died at 20 years as a result of an epileptic seizure. Her seizures had begun in childhood and remained uncontrolled.

Conclusions: Our observations support experience suggesting separate lateralized pathways controlling voluntary and emotional lower facial movements, or a cortical-level disconnection related to epilepsy.

\section{H-03}

\section{Animated electrical source imaging of epileptic events in the} scalp EEG

DW Gross*, ZJ Koles, (Edmonton, AB)

Background: The scalp EEG provides poor localizing information about the origins of epileptic events inside the brain due to the blurring effect of the skull. To reduce this blurring, we have applied the method of Electrical Source Imaging (ESI) to the individual time slices in interictal events in the EEGs of three patients with focal epilepsy.

Methods: Scalp EEGs were recorded from the patients using 27 electrodes and digitally sampled at the rate of 256 per second. An ESI was obtained at each time slice during selected events and the images assembled to produce an animated video of each event. The Talairach head model with a spatial resolution of $7 \mathrm{~mm}$ was used for each patient.

Results: Comparison of ESIs with the voltage topography on the scalp showed significantly more focused activity on the cortical surface than on the scalp. Animation enabled the focus of each event to be identified. The ESI foci were concordant with ictal semiology and, in one case, ictal SPECT.

Conclusions: Animated ESI provides valuable information in localizing the sources of epileptic events in the EEG. Its accuracy will be improved with higher resolution head models derived from the MRIs of individual patients. 


\section{H-04}

Prevalence of and reasons for marijuana use in patients with epilepsy

DW Gross*, N Ashworth, D Quigley, SN Ahmed, (Edmonton, AB)

Background: Currently there is little evidence of a beneficial effect of marijuana on epilepsy. A recent survey of the general population reported that $8.9 \%$ of respondents had used marijuana in the last year, with $1.9 \%$ reporting use for medical reasons. The purpose of this study was to determine the prevalence of and reasons for marijuana use in patients with epilepsy.

Methods: Patients from the University of Alberta Epilepsy Program were questioned through a standardized telephone survey.

Results: Forty-five of 53 subjects responded. Of those, 58\% of subjects report having ever used marijuana with $33 \%$ reporting use in the last year, $22 \%$ monthly use and $20 \%$ weekly use. Seven per cent met DSM IVTR criteria for marijuana dependence. No significant difference in sex or age was observed between active users (use in the last year) and those that were not. For active users, $60 \%$ specifically reported using marijuana for medical reasons (none of which had official approval).

Conclusions: The prevalence of marijuana in this selected population is far higher than that of the general population. The majority of these patients feel it to be beneficial and are using for medical reasons. There is clearly a need to evaluate the efficacy of marijuana on epilepsy in a controlled manner.

\section{H-05}

\section{Complex partial seizures and the "fat" hippocampus - a new entity?}

T Myles*, C Dunham, L Partlo, W Murphy, N Pillay, R Sevick, (Calgary, $A B)$

Background: Transient postictal magnetic resonance imaging (MRI) abnormalities have been described, usually after status epilepticus. Patients in this study all had complex partial seizures (cps), T2 hyperintensity, mass effect and in two cases, gadolinium (gad) enhancement on MR scans, leading to an MR diagnosis of probable medial temporal lobe glioma.

Methods: Combined retrospective and longitudinal study of 10 patients with cps and a "fat" hippocampus and amygdala on MR imaging seen at Foothills Medical Centre in the past five years.

Results: Study included six females and four males; average age at MR diagnosis was 34 years (range 16-56). MR changes were on the left in seven and the right in three. Eight had recent onset of nocturnal generalized seizures, while two had cps for years before the MR diagnosis. All 10 complained of memory difficulty, but neuropsychological testing showed deficits strongly concordant with the MR lesion in only three. EEG localized the seizure onset to the side of the abnormality in four and was nonspecific in six. Each patient had an average of $3.5 \mathrm{MR}$ scans (range 2-7) over a mean time interval of 3.3 years (range 0.5-8). In 3, including one with gad enhancement, the MR abnormalities resolved with control of seizures but seven did not change. Two patients have had removal of the involved amygdala and hippocampus, with improvement in seizure control, but no evidence of tumor. Surgical removal is planned for two other patients who are refractory to medication, while three are being followed with serial examinations and MR scans.

Conclusions: This entity of cps, nocturnal generalized seizures, $\mathrm{T} 2$ hyperintensity and mass effect in the medial temporal lobe, with or without gad enhancement, has not previously been described. Clinicians and radiologists need to be aware that the change may reflect alteration of the blood-brain barrier rather than tumor.

\section{H-06}

\section{MEG improves EEG localization in frontal lobe epilepsy}

F Quesney*, (Madrid, Spain, Montreal, ON); C Amo, T Ortiz, F Maestu, A Fernandez, (Madrid, Spain)

Background: We performed MEG studies in 20 patients with frontal lobe epilepsy who previously underwent extensive EEG investigation and MRI. We compared the localizing effectiveness of MEG, EEG and MRI.

Methods: 20 patients, mean age 21.5 years, underwent simultaneous MEG (148 channels) and 32 channels EEG recordings.

Results: MEG detected epileptic activity in 20 patients and EEG only in 16. In all four patients with normal EEG, MEG localized epileptic abnormality in one frontal lobe. Bifrontal EEG spiking alone or with some temporal lobe involvement was seen in 13 patients of which MEG achieved localization or lateralization of the interictal spiking in 12. In cases with widespread frontotemporal spikes (n: 2), MEG localized the epileptic abnormality in one frontal lobe. MRI lesions were found in eight patients of this series and MEG findings co-localized with the MRI pathology $100 \%$. Whereas EEG results agreed with MRI in only one patient (12.5\%), MEG and EEG colocalized spikes in one patient.

Conclusions: Despite widespread lateralized or bilateral epileptic EEG findings, MEG achieves reliable localization or lateralization of the epileptic abnormality in 19/20 patients with FLE (95\%). MEG results agreed $100 \%$ with MRI findings. MEG can detect frontal lobe spikes that EEG fails to record.

\section{General Neurosurgery I}

\section{I-01}

Factors for preservation of cochlear function in $\mathbf{1 2 5}$ acoustic neuromas with preoperative serviceable hearing

G Mohr*, JJ Dufour, J Rappaport, A Zeitouni, (Montreal, QC)

Background: During the past 21 years $(1981$ - 2002) we have treated 363 patients with acoustic neuromas in oto-neurosurgical collaboration.

Material and Methods: In 125 cases (34.5\%) attempt at hearing preservation was made using intra-operative monitoring and retrosigmoid-transmeatal approach. Size was assessed according to extrameatal diameter parallel to axis of petrous bone. Tumor extension within IAC was assessed in percentage of filling as to longest longitudinal dimension of the canal.

Results: Overall useful hearing preservation was achieved in 30 cases $(23.6 \%)$ for all sizes. Serviceable hearing was obtained in $57.1 \%$ of lesions less than $5 \mathrm{~mm}$ extrameatal diameter, in $41.2 \%$ of lesions less than $10 \mathrm{~mm}$ and in $39.4 \%$ of lesions less than $15 \mathrm{~mm}$. Quality of preoperative hearing: serviceable postoperative hearing 
was obtained in $44.9 \%$ of 49 lesions less than $15 \mathrm{~mm}$ with excellent preoperative hearing (Class 1 Gardner-Robertson: SRT <30dB, SDS $>70 \%$ ) as compared to only $29.1 \%$ of 24 patients with moderate preoperative hearing (Class II Gardner-Robertson: SRT $>30<50 \mathrm{~dB}$, SDS $>50<70 \%$ ). Extent of filling of IAC: in 62 lesions less than $15 \mathrm{~mm}$ in diameter a serviceable hearing was obtained in $54 \%$ of patients with partial filling (0-90\%) and only in $24 \%$ of cases with complete $(100 \%)$ filling of the IAC.

Conclusions: Positive predicting factors for hearing preservation include tumor size below $1.5 \mathrm{~cm}$, excellent preoperative hearing parameters (Class I Gardner-Robertson) and less than $90 \%$ filling of IAC for the intracanalicular tumor component.

\section{I-02}

\section{Robotic long-distance telementoring in neurosurgery}

I Mendez*, DB Clarke, R Hill, I Fleetwood, (Halifax, NS); $S$ Walling, G Kolyvas, (Saint John, NB)

Background: Robots are being used increasingly in minimally invasive cardiac and general surgery. Neurosurgical applications are the next logical step in developing surgical robotic technology. Robotic telecollaboration is the first of a two-phase program to establish telerobotics in neurosurgery at our institution.

Methods:The Socrates telementoring robotic system (Computer Motion Inc.) was used for remote control of an endoscopic camera in the operating field. Four high-speed telecommunication ISDN lines were employed for long-distance transmission of video, voice and navigational data. The mentors had real-time control of a robotic arm capable of manipulating an endoscopic camera in the operating field. A remote controlled multi-view camera provided two-way video and voice input of the operating room environment. Real-time data from an intra-operative navigational system were also available during the procedures.

Results: The robotic telementoring system has been used for long-distance tele-collaboration between two institutions 400 kilometers apart (Halifax and Saint John). Procedures, robotic and telecommunications protocols, have been employed successfully in the excision of a right temporal glioma, resection of a left parietal occipital arterio venous malformation and decompression of the lumbar spine. The input of the mentors was considered very helpful by the neurosurgeons performing the operations.

Conclusions: This initial experience gained with long-distance robotic telecollaboration establishes the proof of principle that longdistance mentoring in neurosurgery is feasible. There is great potential benefit in bringing neurosurgical expertise in real-time to smaller centers. Short-distance robotic teleneurosurgical applications in resident training currently being developed at our institution also have great promise. Although robotic teleneurosurgery is in its infancy, it is expected that this technology will be part of the routine armamentarium of neurosurgeons in the future.

\section{$\mathbf{I - 0 3}$}

\section{NeuroArm: an ambidextrous, microneurosurgical robot}

GR Sutherland*, PM McBeth, DF Louw, (Calgary, AB)

Background: Magnification and advanced imaging have pushed surgeons to the limit of their dexterity and stamina. Robots, in contrast, are indefatigable and have superior spatial resolution and geometric accuracy. NeuroArm is a new ambidextrous robot designed to enhance neurosurgery by enabling precise, tremor-free tool manipulation during microsurgery.

Methods: We approached a company with exceptional experience in space robotics to design an appropriate system. Their engineers spent several months recording and deconstructing surgical procedures into their fundamental components. Finally, a systematic approach was adhered to for the design and breadboard testing of neuroArm.

Results: The system is based on "master-slave" control architecture allowing the robot to function as an assistant or primary surgeon. The robot consists of two articulated arms that grasp and move surgical tools attached to the arm end-effectors. Standard tools such as forceps, needle drivers, suction, micro-scissors and dissectors interface the end-effector with its target. For precise tool positioning each arm has 8-DOF and a 3-DOF optical force sensor to provide the surgeon with haptic force feedback.

Conclusions: Breadboard testing has shown the prototype to have greater spatial resolution than requested by the end users. The system promises to enhance surgical performance, reduce fatigue, and improve surgical outcomes.

\section{I-04}

\section{Results with the Jannetta procedure}

AM Kaufmann*, (Winnipeg, MB)

Background: Microvascular decompression (MVD) surgery was introduced in 1968 and subsequently further refined by Dr. Peter J. Jannetta. The author presents his own experience with performing this procedure.

Methods: The surgical observations, complications and outcomes have been prospectively followed in a consecutive series of MVD operations (1996-2002).

Results: The 320 cases included presenting diagnoses of trigeminal neuralgia, hemifacial spasm and other cranial nerve disorders. Intraoperative monitoring was routinely employed. The average surgery time was less than two hours, and hospitalization $3.4 \pm 1.5$ days. The symptomatic neurovascular compression was always found, usually prominent vertebrobasilar artery branches, and isolated veins in less than $10 \%$ of cases. Major operative complications were rare: $1 \%$ hearing loss; $0.3 \%$ stroke (venous infarct - good recovery) and no hematomas or death. Delayed complications requiring readmission included: $3 \%$ aspectic meningitis, $1 \%$ each of CSF leak, wound infection and other. Annual follow-up results have been excellent/good in $80 \%$ with trigeminal neuralgia and $90 \%$ with hemifacial spasm.

Conclusions: Good results and minimal complication risk may be achieved with MVD surgery.

\section{I-05}

\section{The feasibility of a gamma knife stereotactic radiosurgery} centre in Canada

\section{AM Kaufmann*, M West, (Winnipeg, MB)}

Background: Lars Leksell described stereotactic radiosurgery (SRS) as a method to destroy intracranial targets using single high doses of focused ionizing radiation, administered using stereotactic guidance. Over 1,500 published studies support the use of Gamma 
Knife (GK) SRS, and over 155 units worldwide have been installed. However, there are no Canadian GK centres, at least in part due to the significant up-front investment in the equipment itself.

Methods: The feasibility of establishing a GK-SRS program in Canada was assessed, using estimates of program development/ operation costs and potential clinical throughput.

Results: An estimated 280 Manitobans and 6,700 Canadians would be candidates for GK-SRS annually. The average number GK-SRS procedures performed by units around the world is 218 per year, with some centers treating as many as 600 patients annually. We assumed conservative utilization estimates of 120 cases the first year, and 240 annually thereafter. The capital and operating costs were budgeted, resulting in favorable cost per procedure estimated for GK-SRS. While alternative LINAC bases SRS requires less initial capital costs than needed to establish a GK facility, the significantly lower patient throughput limits potential cost savings (e.g. 30 annual cases with LINAC-SRS compared to 240 with GKSRS).

Conclusions: Published outcome studies support the incorporation of SRS into routine neurosurgical practice. Our projections indicate that establishing GK-SRS programs in Canada is financially feasible and will afford overall improvement of patient care.

\section{I-06}

\section{Posterior pituitary bright spot and location of residual adenohypophysis in intra and suprasellar lesions on MRI}

B Sade*, G Mohr, JL Vezina, (Montreal, QC)

Background: This study was undertaken to assess the radiological modifications and shifts of the normal pituitary gland on MRI and to determine if the position of the Bright Spot (BS) (neurohypophysis) represents a predicting factor for the position of the residual adenohypophysis in pathological conditions.

Methods: In 94 patients with intra or suprasellar pathologies, presence and respective topography of the BS and adenohypophysis were scrutinized on MRI, according to lesion type, size, endocrine status and intra-operative findings in the surgical group. In a control group of 102 patients without pituitary pathology, the presence of BS was evaluated.

Results: In the normal control group, a BS was visible in $91.2 \%$ of 102 cases; in 94 patients with sellar lesions, it was present in 70 (74.5\%), and the location of the adenohypophysis was felt to be clearly identified in 52 patients $(55.3 \%)$, regardless of the presence of a BS The adenohypophysis was identified in 45 cases $(64.3 \%)$ in BS positive patients, whereas it was only found in 7 (29.2\%) of 24 BS negative.

Conclusions: The BS can be identified in the majority and the residual adenohypophysis in more than half of cases with pituitary lesions. We feel that the position of the BS helps predict the location of the residual functional adenohypophysis, thus contributing to preservation of the pituitary gland during surgical procedures.
I-07

Routine intra-operative monitoring improves neurosurgical outcome

$M$ Wilkinson*, S Chan, M Kozey-Rogers, AM Kaufmann, (Winnipeg, $M B$ )

Background: There is a risk to hearing with any posterior fossa surgery. We report our experience with intra-operative monitoring (IOM) during microvascular decompression (MVD) surgery.

Methods: We reviewed a consecutive series of 240 MVD surgeries performed by one surgeon (1997-2002 AMK). Brainstem auditory evoked responses (BAER) were recorded throughout MVD procedures and quantitative analysis was performed on 124 recordings available for retrospective review.

Findings: Mean latency change was 0.80 .5 milliseconds (ms). Maximal latency changes were less than $1 \mathrm{~ms}$ in $66 \%$ and $1.0-1.9 \mathrm{~ms}$ in $28 \%$. Changes of $2 \mathrm{~ms}$, seen in $6 \%$, were associated with loss of recognizable waveforms. Surgical maneuvers to alter retraction, release arachnoid tethers or alleviate vascular compression upon the cochlear nerve were successful in retrieving BAER and reducing latency delays in all cases. One patient $(0.5 \%)$ had postoperative loss of hearing (ipsilateral), and in that case the BAER waveforms were not measurable prior to MVD. There was only one other serious postoperative complication; venous infarct with good recovery.

Conclusions: We believe routine IOM with attention to minimizing BAER changes is associated with a low incidence of serious neurological complications in MVD surgery.

\section{I-08}

Endoscopic removal of colloid cysts of the third ventricle: the McMaster experience

A Cenic*, K Reddy, (Hamilton, ON)

Background: Colloid cysts represent $1 \%$ of primary brain tumors. In this study, we review our experience with the endoscopic surgical treatment of colloid cysts, and compare it to available historical data on the results of open microsurgical approaches and those of endoscopic approaches previously published.

Methods: Between April 1997 and April 2002, 11 patients underwent endoscopic surgery in attempts to treat their colloid cysts. Two cases were converted to open microsurgical approach because of venous bleeding impeding visibility. Uniportal right frontal horn approach was undertaken in all cases. Post-op CT or MRI was acquired in all patients.

Results: Three out of the 11 patients experienced postoperative complications that appeared not directly related to the surgical procedure. The primary presenting symptom of headache resolved completely postoperatively in $90 \%$ of the patients, and initial MRI or CT postop scans revealed greater than $50 \%$ resection of cyst in five patients. Mean operating time was 113 minutes, and mean number of postoperative length of stay was 2.8 days. These results appear to demonstrate lower morbidity in comparison to results of the traditional approaches, and compare favorably with the results of other published endoscopic series.

Conclusions: Total or near-total resection of colloid cysts can be achieved with endoscopy, with little morbidity, shortened operative time, reduced length of stay, and resolution of symptoms. 


\section{Multiple Sclerosis / Dementia}

\section{$\mathrm{J}-\mathbf{0 1}$}

\section{Evidence for cortical grey matter dysfunction in multiple} sclerosis: MEG findings

F Quesney*, (Montreal, ON, Madrid, Spain); C Amo, T Ortiz, A

Fernandez, F Maestu, S Fernandez, (Madrid, Spain)

Background: Although MRI can identify cortical grey matter lesions in multiple sclerosis (MS) patients, this seldom occurs and therefore further confirmation of this finding by other neuroimaging techniques, such as MEG, seems advisable.

Methods: We performed simultaneous MEG and EEG recordings with a 148 channels MEG device on 22 patients with relapsing remitting MS and at least two relapses within the past two years. Average age (32 years), mean disease duration 3.2 years and EDSS score 1.7. All patients presented with typical MRI lesions.

Results: MEG spikes were recorded in 13/22 patients and in four of them they were paroxysmal. Spikes were localized in the ascendent parietal gyrus and other parietal areas (16 cases), as well as in temporal and frontal regions (10 cases). MEG tracings showed widespread theta slow waves in $19 / 22$ patients, specially in parietal and temporal areas. There was a close spatial correlation between the MEG findings and MRI lesions.

Conclusions: We propose that the paroxysmal MEG activity recorded in patients with MS, represents an intrinsic cortical grey matter dysfunction which may help us to further understand MS paroxysmal symptomatology and MS relationship with epilepsy. The slow wave activity is the cortical expression of underlying white matter lesion.

\section{$\mathbf{J - 0 2}$}

\section{Serum immunoglobulin levels in MS patients prescribed glatiramer acetate with or without pretreatment with an interferon- $\beta$ in clinical practice}

\section{H Tremlett*, J Porciuncula, J Oger, (Vancouver, BC)}

Background: Glatiramer acetate (GA) stimulates a humoral immune response, of which immunoglobulins (Ig) are an important component. Elevated IgG levels during treatment with GA have been associated with a higher proportion of relapse free patients. We investigated serum Ig levels in GA treated MS patients and ascertained if a previous prescription of IFN $\beta$ influenced results.

Methods: A retrospective review of serum IgG, $\operatorname{IgM}$ and $\operatorname{IgAwas}$ carried out in a group of MS patients treated with GA at the MS clinic, UBC. Immunoglobulins were measured pretreatment, at month 1, 3, 6 and yearly thereafter.

Results: A total of $91 \mathrm{MS}$ patients were eligible, 86 charts were available for review. Of these, $46 / 86$ had one or more Ig result. Mean time on drug was 332 days. Serum $\operatorname{IgM}$ and $\operatorname{IgA}$ levels remained relatively constant throughout treatment. Few (2) patients with normal pretreatment Igs developed Ig levels outside of the normal range. However, IgG levels increased during treatment, peaking at months 3-6; this was significantly greater than at pretreatment (paired t-test, $\mathrm{p}=0.0088$ ). Patients previously prescribed an IFN $\beta$ $(\mathrm{n}=23)$ had lower IgG levels, than those not previously prescribed an IFN $\beta$, which was significant at months $>0$ to $3(\mathrm{p}=0.026)$.

Conclusions: There were significant changes in serum IgG in patients treated with GA. Patients previously prescribed an IFN $\beta$ responded differently to GAthan those who had not. These patients may have an altered (IgG) immune response because of: 1) pretreatment with an IFN $\beta$ or 2) an underlying difference which caused intolerance or nonresponse to IFN $\beta$ and necessitated a switch to GA. This phenomenon should be studied further, especially with regards to clinical efficacy.

\section{$\mathbf{J - 0 3}$}

Multiple sclerosis registry for pregnancy outcome and longitudinal follow-up of offspring

AD Sadovnick*, (Vancouver, BC); P Duquette, (Montreal, QC)

With the advent of improved diagnostic procedures and criteria, the lagtime between onset and diagnosis of multiple sclerosis (MS) has reduced dramatically. Young adults are receiving the diagnosis very early in the disease course, often when the phenotype is relatively mild or even benign. Early MS treatment is now becoming the norm.

As far as we know, there has been no objective, systematic follow-up of pregnancy outcome if a parent has MS or of the longitudinal follow-up of the resultant offspring with respect to behavioral and immunological implications. Thus, we believe a Canadian Registry to look at these issues is warranted, feasible, and timely. We have taken preliminary steps to obtain unbiased funding and to develop a possible advisory panel. The purpose of this presentation is to put forward the concept of this registry to community neurologists. The success of the registry is dependent on the inclusion of as representative a sample as possible.

The registry data should provide objective information on pregnancy outcome according to therapeutic profiles, the impact of breast-feeding and the longitudinal developmental and immunological assessment of the resultant infants. Comparisons will be made as appropriate with age and ethnically matched controls, data for who are available in the public domain.

\section{J-04}

\section{Premenstrual MS pseudoexacerbations ameliorated by aspirin}

D Wingerchuk*, (Scottsdale, AZ)

Background: Some women with multiple sclerosis (MS) experience premenstrual worsening of fatigue or transient focal neurological symptoms. The pathophysiology of these symptoms is not established.

Methods: Case series derived from MS subspecialty clinic.

Results: Three women with relapsing-remitting MS experienced stereotypical self-limited premenstrual symptoms. Patient 1, aged 29 and baseline Expanded Disability Status Scale (EDSS) score 2.5, experienced 2-3 days of severe fatigue, bilateral leg paresthesias and right leg weakness a week prior to her period for 11 months. Aspirin $650 \mathrm{mg}$ BID eliminated the events. Patient 2, aged 33 and EDSS 1.0, noted 3-4 days of fatigue and intermittent diplopia 9-12 days premenses; aspirin $325 \mathrm{mg}$ BID reduced fatigue and prevented diplopia. Patient 3, aged 36 and EDSS 1.5, experienced 4-7 days of premenstrual fatigue, asymmetric leg weakness, and urinary urgency that responded completely to aspirin $650 \mathrm{mg}$ BID. A slight increase in morning body temperature was sometimes associated with the symptoms in $2 / 3$ women but was not affected by aspirin treatment. 
Conclusions: Premenstrual pseudoexacerbations may be stereotypic, prolonged, and associated with fatigue and possibly increased body temperature. The response to aspirin therapy suggests that neuroendocrine mechanisms separate from effects on core body temperature may generate such symptoms.

\section{$\mathrm{J}-\mathbf{0 5}$}

MEG slow wave activity correlates with MRI hippocampal atrophy in Alzheimer's disease: diagnostic implications

A Fernandez, J Arrazola, F Maestu, C Amo, T Ortiz*, (Madrid, Spain); F Quesney, (Madrid, Spain, Montreal, ON)

Background: Patients suffering from Alzheimer's disease (AD) show enhanced slow wave activity in conventional scalp EEGs. This is associated with increased MEG dipole density in the low frequency band. The slow wave activity concurs with atrophy and reduced metabolic rates mainly in temporoparietal structures. The relationship between MEG and structural changes in AD and their diagnostic implications are assessed in this study.

Methods: Whole-head magnetoencephalographic recordings were obtained from 15 right-handed AD patients (mean age: 76 years) fulfilling NINCDS/ADRDA diagnostic criteria and 16 healthy control subjects (mean age: 74 years). MRI volumetric studies (1.5 Tesla) were performed, including global cerebral, temporal lobe and hippocampal volumes.

Results: Slow wave dipole density (DD) in the delta and theta bands was enhanced in the AD group, particularly in temporoparietal regions, as compared to healthy controls. Left hippocampus MRI atrophy was associated with left temporal theta DD in 14 out of 15 AD patients. This was statistically significant in a discriminant analysis.

Conclusions: MRI, MEG coregistration improves diagnosis in patients with $\mathrm{AD}$ and it may help us to further understand the pathophysiology of this condition. The yield of this procedure in obtaining early $\mathrm{AD}$ diagnosis appears promising.

\section{$\mathbf{J - 0 6}$}

Sensitivity and specificity of the Montreal cognitive assessment (MOCA) as a cognitive screening tool for detection of mild cognitive deficits

Z Nasreddine*, I Collin, (Greenfield Park, QC); H Chertkow, $N$ Phillips, H Bergman, $V$ Whitehead, (Montreal, QC)

Background: Mild cognitive impairment (MCI) is associated with high annual conversion rate to Alzheimer's disease, but no adequate screening tests exist to detect it. We developed the Montreal Cognitive Assessment (MOCA) as a screening tool to detect MCI.

Methods: 13 patients with Alzheimer disease (AD), and 30 meeting criteria for MCI were compared to 34 healthy elderly controls. The MMSE and the MOCA were administered to both groups. The MOCAis a one-page 30-point scale assessing memory and eight other cognitive domains. A score less than 25 is considered abnormal.

Results: All AD patients scored below 25 on the MOCA. The MCI group all had MMSE of 25 or greater (mean 27.5). Normals all scored 25 or more on the MMSE, and had a mean of 27 on MOCA.
Using a cut-off score $<25$ on the MOCA, the sensitivity and specificity of the MOCAto distinguish abnormal performance in the MCI group, compared to the normal controls, was $80 \%$. The specificity was $91 \%$.

Conclusions: With a high sensitivity and acceptable specificity, the MOCA may be a useful brief cognitive screening tool for detection of mild cognitive deficits. A score below 25 on the MOCA has a high specificity for MCI.

\section{J-07}

Lewy body and frontotemporal dementia groups are more variable than those with mild cognitive impairment: results from the Quebec Dementia Registry

H Chertkow*, A Robillard, F Massoud, D Tessier, D Goupil, (Montreal, QC); L Verret, R Bouchard, (Quebec, QC)

Background: The Cognition group of the FRSQ Aging Network (Reseau en Vieillessement) established a province-wide Registry last year to collect data on Lewy Body Dementia (LBD), Frontotemporal Dementia (FTD) and Mild Cognitive Impairment (MCI). One question relevant to organizing drug studies is whether these groups differ in terms of variability.

Methods: Patients with LBD $(\mathrm{n}=15)$, FTD $(\mathrm{n}=15)$, and MCI $(n=15)$ were assessed at 13 different Memory clinics across the province with standardized clinical, neuropsychological, and imaging tools, and databased centrally. Tests of cognition, function, behavior, depression, subjective complaints, global severity, along with demographic data were collected.

Results: On virtually all measures, the MCI patients represented a more coherent group with a significantly lower standard deviation. On measures of function and behavior they were at ceiling, however.

Conclusions: Drug studies of LBD and FTD will have to enroll greater numbers of subjects to compensate for the apparent wider range of cognitive and behavioral impairment compared to MCI groups.

\section{$\mathbf{J}-\mathbf{0 8}$}

Alzheimer's disease presenting as motor apraxia: MRI and pathological evidence for diffuse cortical involvement

R Camicioli*, (Edmonton, AB); J Quinn, J Kaye, G Murdoch, $M$ Ball, M Moore, (Portland, Oregon)

Background: Progressive apraxia is a prominent, early manifestation of corticobasal degeneration (CBD) and rarely Alzheimer's disease (AD) and other dementias. Our objective is to report two men with progressive apraxia with autopsy proven AD (AAD) and premortem volumetric MRI.

Methods: Clinico-pathological correlation with in vivo volumetric MRI.

Results: One patient presented at age 62 years with right arm apraxia and myoclonus, followed by progressive personality and cognitive change, and death at age 67 . The second presented with myoclonus at age 56 followed by global cognitive decline, including apraxia, and death at 62 years. Differential diagnosis in both was AD vs CBD. Longitudinal quantitative volumetric MRI was compared to five sex- and age-matched AD patients and five controls. Cortical volume/intracranial volume (ICV) ratio was smaller and 
subarachnoid space/ICVratio was larger ( $>1 \mathrm{SD})$ in AAD than both comparison groups. Hippocampi did not differ from typical AD. Pathology showed marked, diffuse tangle-predominant cortical involvement that included motor areas.

Conclusions: Apraxia occurs as an initial and predominant manifestation of $\mathrm{AD}$ and is associated with aggressive cortical (including motor area) involvement. While consistent with previous clinical pathological correlations, this was evident by volumetric MRI. Diagnosis in life is critical in anticipation of disease-specific therapies.

\section{NEUROPHYSIOLOGY}

\section{K-01}

Involvement of the human subthalamic nucleus in movement preparation

Guillermo Paradiso*, Jean A Saint-Cyr, Andres M Lozano, Anthony E Lang, Robert Chen (Toronto, ON)

Winner of the Canadian Society for Clinical Neurophysiology's Herbert Jasper Prize. See page 10.

\section{K-02}

Callosal drift: a new sign supporting existence of 1-way callosal traffic underpinning laterality of movement control in humans

\section{Derakhshan*, (Charleston, WV)}

I present digitized optokinetic videos of two right-handed patients with vascular callosal lesions in whom the dynamic de-activating influence of the major hemisphere upon the cortical motor apparatus of the minor was demonstrated, as follows: Voluntary flexion and extension of the outstretched right arm at the elbow (when pointing to the nose and back to the original posture) and wrist (with cocking up the wrist, then back to original position) resulted in sudden down-ward drift of the outstretched left arm, followed by its return to the original position at each of the two movement sequences occurring at elbow and wrist, within a time commensurate with the inter-hemispheric transfer time (60-100 $\mathrm{msec}$ ). Similar movements on the left did not affect the posture of the outstretched right arm. Both patients underwent MRI of the brain with diffusion weighted imaging, showing mild involvement of the subcortical mesial frontal regions within the territory of the right anterior cerebral artery. A comprehensive review of the literature will be presented, supporting the proposed 1-way callosal traffic theory underpinning lateralities of executive functions in humans, affecting all movements including those of the eyes and voice.

\section{K-03}

Percentage of maximum muscle potential evoked after transcranial electric stimulation in anaesthetized patients

D Houlden*, M Vennettilli, D Rowed, M Schwartz, M Fazl, A Taylor, R Midha, (Toronto, ON)

Background: Motor evoked potentials (MEPs) after transcranial electric stimulation (TCES) are used to assess motor pathways during surgery, but the percent of motor pathways they assess is not known.
Methods: MEPs were recorded from tibialis anterior (TA) and plantar foot (PF) muscles after TCES (Digitimer D185 stimulator). Maximum muscle (M) potentials were recorded from the same muscles after supramaximal stimulation of their respective peripheral nerves. The peak-to-peak MEPamplitude was divided by the maximum M-potential amplitude for each muscle, then multiplied by 100 to determine the percent of maximum M-potential represented by each MEP.

Results: Six anaesthetized patients with normal pre-operative lower limb function had MEPs and maximum M-potentials recorded from 9 TA and 11 PF muscles. The mean MEP amplitude (and SD) from TA and PF muscles was $0.16 \mathrm{mV}(0.11)$ and $0.81 \mathrm{mV}(0.47)$ respectively. The mean maximum M-potential amplitude from TA and $\mathrm{PF}$ muscles was $4.8 \mathrm{mV}(1.5)$ and $13.6 \mathrm{mV}$ (5.5) respectively. The mean percent of maximum M-potential amplitude for MEPs was 4 percent (2.3) for TA and 6.8 percent (4.2) for PF muscles.

Conclusions: TCES evoked, on average, less than 10 percent of the total membrane current available from TA and PF muscles. This limitation should be considered when interpreting MEPs in anaesthetized patients.

\section{K-04}

Gender effects of serotonin depletion in experimental atypical absence seizures

E Bercovici*, V Persad, MA Cortez, OC Snead III, (Toronto, ON)

Background: Bercovici, et al reported that serotonin depletion with parachlorophenylalanine (PCPA) protected against atypical absence seizures in AY-9944 rats (Can J Neurol Sci 2001; 28: S11). Whether PCPA treatment is equally effective in males and females is unknown.

Methods: Long-Evans hooded rats $(\mathrm{N}=29)$ received AY-9944 and chronic monopolar epidural electrodes for sequential EEGs, one day before and one day after three doses of PCPA methyl-ester treatment at 10:00 hours. Vaginal smears were taken for the duration of experiment before injections to control for changes in estrus cycle.

Results: Total slow spike-and-wave discharge (SSWD) duration (mean SEM) dropped from 484.4263 .98 to 259.4213 .48 , in males (46.45\%), $(\mathrm{n}=6, \mathrm{p}<0.01)$; SSWD duration decreased from 683.69 138.60 to 265.1969 .45 , in females $(61.21 \%)$, $(n=8, p<0.05)$; nonsignificant change in SSWD duration in their respective controls ( $\mathrm{n}$ $=8,7)$. PCPA treatment reduced the weights in male AY $(\mathrm{p}<0.005)$, male control ( $\mathrm{p}<0.001)$, female AY ( $\mathrm{p}<0.001)$ and female control ( $\mathrm{p}$ $<0.05$ ). More than $75 \%$ of all females were cycling after PCPA treatment.

Conclusions: PCPA protected against experimental atypical absence seizures more effectively in females compared to males.

\section{K-05}

The brain adapts the vestibulo-ocular reflex after monocular peripheral ocular motor nerve palsies: motor learning to violate Hering's law

James Sharpe*, Agnes MF Wong, Douglas Tweed, (Toronto, ON)

Background: Paresis of a muscle might be expected to lower vestibulo-ocular reflex (VOR) responses in its field of motion. Hering suggested that the brain circuitry controlling eye movements 
consists of two systems, one for conjugate movements, the other for vergence. We investigated a conjugate subsystem, the VOR, in patients with unilateral sixth, third or fourth nerve palsies.

Methods: Patients with unilateral sixth $(\mathrm{n}=21)$, third $(\mathrm{n}=10)$ or fourth nerve $(n=13)$ palsy and 15 normal subjects were studied. Subjects made active sinusoidal head on body rotations in yaw and pitch at approximately 0.5 and $2 \mathrm{~Hz}$, and in roll at approximately 0.5 , 1 and $2 \mathrm{~Hz}$. Eye movement were recorded by 3D binocular magnetic scleral search coils in darkness and during monocular viewing with each eye in light.

Results: In sixth nerve palsy horizontal VOR gains (eye speed/head speed) in darkness were decreased in the paretic eye in both abduction and adduction, but remained normal in the nonparetic eye in both directions. In light, horizontal visually enhanced VOR (VVOR) gains were normal in both eyes in moderate and mild palsy. In severe palsy, horizontal VVOR gains remained low in the paretic eye during viewing with either eye, while those in the nonparetic eye were higher than normal when the paretic eye viewed. Vertical VOR and VVOR were normal, but dynamic and static torsional VOR and VVOR gains were reduced in both eyes in all patients. In third nerve palsy horizontal VOR and visually enhanced VOR (VVOR) gains of the paretic eye were decreased during both abduction and adduction. Vertical VOR and VVOR gains of the paretic eye were decreased during both elevation and depression. Dynamic and static torsional VOR and VVOR gains of the paretic eye were reduced during both excyclotorsion and incyclotorsion. Horizontal, vertical and torsional VOR and VVOR gains were normal in the nonparetic eye. In fourth nerve palsy dynamic torsional VOR (in darkness) and visually enhanced VOR (VVOR) gains of the paretic eye were decreased during both incyclotorsion and excyclotorsion, whereas gains in the nonparetic eye were normal. Vertical VOR gains of the paretic eye in darkness were decreased during elevation and depression; they were normal in the nonparetic eye. In light, vertical VVOR gains were normal in both eyes. Horizontal VOR gains of the paretic eye in darkness were decreased during abduction and adduction, whereas horizontal gains in the nonparetic eye were normal. In light, horizontal VVOR gains were normal in both eyes.

Conclusions: Motion of the eyes after nerve palsies exemplify monocular adaptation of the VOR in three dimensions. During head rotation in darkness, VOR gains are reduced during movement both in the direction of the paretic muscle and in the direction of its antagonist. VOR gains in the nonparetic eye remain normal, indicating a selective central adjustment of innervation to the paretic eye, and specifically to the antagonists of paretic muscles. In light, torsional VVOR gains in the paretic eye remain reduced in third and fourth nerve palsies. Visual input increases vertical and horizontal VVOR gains to normal in the paretic eye in third and fourth nerve palsy, without a conjugate increase in VVOR gains in the nonparetic eye; this provides further evidence of selective adaptation in the paretic eye, in violation of Hering's law.

Supported by Canadian Institutes of Health Research (CIHR) Grants MT 15362 and ME 5504

\section{K-06}

Recovery from hypoglycemia-induced metabolic perturbation observed using ex vivo $1 \mathrm{H}$ magnetic resonance spectroscopy

\section{$R$ Tyson*, G Sutherland, (Calgary, AB)}

Background: Severe hypoglycemia and hypoglycemic coma are common occurrences associated with insulin treatment of diabetes and can result in neurological impairment. Large metabolic perturbations are apparent, as purine, pyrimidine and amino acid metabolism is grossly altered. This study confirms previous work and adds new insight into metabolic alterations both during hypoglycemic coma and following restitution of normal blood glucose levels.

Methods: All animals used in this study were treated in accordance with guidelines of the Canadian Council on Animal Care and local animal care committees approved all protocols. Male Sprague-Dawley rats (150-170 g) were intubated and mechanically ventilated with $0.7 \%$ halothane and a $2: 1$ mixture of $\mathrm{N}_{2} \mathrm{O} / \mathrm{O}_{2}$. Hypoglycemic coma was induced using an IV infusion of insulin (Actrapid, 1-1.5 IU/kg in total) and EEG monitored throughout using bipolar electrodes placed in the temporalis muscle on either side of the cranium. After $40 \mathrm{~min}$ of EEG silence, $25 \% \mathrm{w} / \mathrm{v}$ glucose was infused IV. At various times (10, 20, 30 and $40 \mathrm{~min}$ coma, or 1, 3,12 and $24 \mathrm{hr}$ recovery) cerebral metabolism was arrested using freeze-funnel fixation and metabolites extracted from frozen samples of neocortex by homogenization in $0.5 \mathrm{M}$ perchloric acid (water-soluble) or 1:1 methanol-chloroform (organic-soluble). Concentrations of metabolites in each sample was determined using 1H NMR spectroscopy and identification of unknown metabolites made using 2-D NMR spectroscopic techniques (COSYand TOCSY spectra).

Results: Hypoglycemic coma results in large changes in purine and amino acid metabolism after $40 \mathrm{~min}$ of EEG silence. Decreases in ATP and GTP, with concurrent increases in GMPand AMP, were observed. Purine metabolism was evident in the presence of IMPand adenosine, and pyrimidine metabolites (uridine and uracil) were also detected. Amino acid concentrations were also significantly altered. Cortex concentrations of glutamate and glutamine were significantly decreased. Linoleic and linolinic acids were abolished by the end of 40 min coma. During recovery, the various metabolites differ in their time for recovery. Energy charge rapidly recovers, but ATP levels remain depressed. Uracil remains elevated for several hours following glucose administration. Glutamate precedes glutamine in returning to control. Fatty acids require between 12 and $24 \mathrm{hr}$ to reappear.

Conclusions: It has been known for some time that purine metabolism is significantly altered during hypoglycemic coma, but this is the first observation that purine degradation also occurs. This has important implications in the recovery of UTP, since there is no recovery mechanism analogous to the purine cycle. Thus, UTPmust be resynthesized from orotate, the rate-determining step in the formation of pyrimidine phosphates. The slow recovery of glutamine relative to glutamate may indicate a nitrogen deficit, since a loss of ammonia is known to occur with energy failure. 


\section{General Neurology II}

Clinical and electrophysiological associations with mortality in anoxic ischemic encephalopathy

B Young*, (Toronto, ON); A Ragazonni, (Florence, Italy); G Doig, (Melbourne, Australia)

Background: Electrophysiological tests hold promise in the prognostication of comatose survivors of cardiac arrest.

Methods: Prospective observational study.

Results: 75 adult patients with cardiac arrest assessed within 48 hours. Corneal reflex absent: 93\% died, present: $60 \%$ died $(\mathrm{p}=0.001)$; pupillary reflex absent: $90 \%$ died, present $70 \%$ died $(\mathrm{p}=0.126)$; oculovestibular reflex absent: $94 \%$ died, present: $72 \%$ died $(\mathrm{p}=0.37)$; pharyngeal reflex absent: $93 \%$ died, present: $64 \%$ died $(\mathrm{p}=0.37)$; motor: no response: $97 \%$ died; purposeful: $38 \%$ died $(\mathrm{p}=0.001)$. EEG: malignant pattern: $94 \%$ died, mild dysrhythmia: 94\% died ( $\mathrm{p}=0000001)$; N20 somatosensory response absent: $95 \%$ died; present: $58 \%$ died $(\mathrm{p}=0.003)$. All who died did not recover consciousness.

Conclusions: No single finding or test has $100 \%$ prediction of mortality without recovery of consciousness. A larger study is needed to investigate tests in parallel or series.

\section{K-08}

Thalamic and hippocampal ibotenic acid lesions in the AYmodel of atypical absence seizures

N Mistry, M A Cortez*, O C Snead III, (Toronto, ON)

Background: AY-9944 (AY) induced slow spike-and-wave discharges (SSWD) emanate from cortex, thalamus and hippocampus. The effect of postnatal lesions of thalamus or hippocampus in AY rats with atypical absence seizures remain to be determined.

Methods: Long Evans hooded rats (LEHR) $(\mathrm{N}=7)$ with two frontal monopolar and bipolar electrodes placed in the dorsal hippocampus (CA3) and nucleus basalis (NB); five LEHRs with two frontal monopolar and two bipolar electrodes placed in the nucleus reticularis thalami (NRT) and the ventroposteromedial nucleus (VPM). One-hour baseline EEG recordings were ascertained for quantification of SSWD. One week later, bilateral injections of ibotenic acid (IBO) $(4 \mu \mathrm{g} / \mu \mathrm{l})$, through an implanted cannula in CA1 or VPM.

Results: Synchronous SSWD from CA3, NB, NRT, VPM and frontal cortices were recorded. Bilateral IBO lesions in VPM attenuated AY-induced SSWD duration (mean + SEM) (274.4 \pm 152.6$)$ compared to prelesion controls (513.8 \pm 165.0$)$. CA1 lesions exacerbated AY-induced SSWD duration (785.3 \pm 350.7$)$ compared to controls (553.7 \pm 311.3$)$. SSWD in lesioned animals were abolished by treatment with ethosuximide $(82.5 \pm 54.8)$, (p. $<0.05$, Student $t$ test).

Conclusions: The thalamocortical circuitry is directly involved in SSWD in the AY model. However, the postnatal CA1 insults lead to an exacerbation of SSWD, although they may be treatable with antiabsence medication.

\section{L-01}

Effectiveness of a multidisciplinary headache program in patients with chronic daily headache

JE Magnusson, WJ Becker*, (Calgary, AB); CM Riess, (Edmonton, $A B)$

Background: Approximately 4\% of the general population experiences chronic daily headache $(\mathrm{CDH})$. Many of these patients experience significant disability and reduced health-related quality of life (HRQoL).

Objective: To review treatment outcomes for patients with $\mathrm{CDH}$ from a multidisciplinary headache program (MDHP) and compare these to a second patient group with $\mathrm{CDH}$ treated in a clinic setting.

Methods: Disability measures (Headache Disability Inventory [HDI]) and HRQoL measures (SF-36) were completed before and after treatment by 36 patients in a MDHP, and by 70 patients treated primarily with pharmacological therapy in a clinic setting. All patients in the clinic group had a diagnosis of transformed migraine, and this was also the most common diagnosis in the MDHPgroup.

Results: The patient group in the MDHP showed significant improvement with treatment on HDI scores $(\mathrm{p}<0.001)$, on scores in all eight domains of the SF-36 $(\mathrm{p}<0.05)$ and on measures of headache intensity. The patient group in the clinic setting showed some improvement in headache frequency, but no significant improvement on HDI scores, SF-36 scores, or measures of headache intensity.

Conclusions: Treatment of patients with severe headache disorders may be more successful in a multidisciplinary headache program as compared to an office setting.

\section{L-02}

From research to policy - developing an integrated stroke strategy for Nova Scotia

G Gubitz*, S Phillips, E Harrison, (Halifax, NS)

Background: Nova Scotia's geography and small population have resulted in inequities in stroke care delivery; evidence-based, locally relevant stroke care is needed for all Nova Scotians.

Methods: With the support of our provincial Heart and Stroke Foundation and Department of Health, a core committee of content experts was assembled representing primary prevention, emergency and acute care, rehabilitation and reintegration, and evaluation / monitoring. Each group had province-wide membership, and, within established terms of reference, developed evidence-based, locally relevant stroke care recommendations.

Results: A formal document has been published (www.heartandstroke.ca), including a needs assessment and the following recommendations for organizing provincial stroke care: 1. Make stroke a top healthcare priority and establish a provincial "Stroke Working Group". 2. Implement an integrated stroke care system with a service delivery model including guidelines for stroke care. 3. Develop comprehensive strategies for stroke prevention, acute care and rehabilitation. 4. Develop a provincial stroke data set and a coordinated public education program. 5. Enhance information and diagnostic (telemedicine) technologies. 
Conclusions: Our document is the result of successful consensusbuilding. It has been disseminated to stakeholders, and presented to the provincial Department of Health, which has appraised and accepted it, and is now assembling a provincial Stroke Working Group, which will develop a business plan. Our methods may be useful to others interested in putting evidence into action.

\section{L-03}

\section{Neuropsychological profile of childhood epilepsy syndromes}

M Nolan*, (Toronto, ON); M Redoblado, S Lah, M Sabaz, J Lawson, A Bye, (Sydney, Australia)

Background: Greater understanding of cognitive abilities in children with epilepsy will assist appropriate educational intervention. We determined the neuropsychological profiles of common epilepsy syndromes - Generalized Idiopathic Epilepsy (GIE) including Childhood Absence Epilepsy (CAE), Temporal Lobe Epilepsy (TLE), Frontal Lobe Epilepsy (FLE), Central Epilepsy (CE), nonlocalized Partial Epilepsy (PE) and Generalized Symptomatic Epilepsy (GSE).

Methods: Syndrome identification was determined by International League Against Epilepsy criteria in 172 children with intractable epilepsy monitored with video-EEG, using clinical data, seizure semiology, interictal and ictal recordings. Neuropsychology assessment included intelligence quotient (IQ) and memory function using age-normed and validated instruments. After adjusting for important epilepsy variables, $95 \%$ confidence intervals were compared for IQ and memory functions between specific syndrome groups.

Results: Analyzing IQ, children with GIE, CE and TLE performed best, and did not differ statistically. Children with GSE had statistically lower IQ than other syndrome groups except PE. Children with FLE functioned significantly better than GSE but did not differ statistically from other groups. Considering memory function, children with TLE had the greatest disabilities. Children with FLE had significant memory difficulties, and children with CAE had only minor disability.

Conclusions: In childhood epilepsy, delineation of syndrome has important implications when considering cognitive potential. This information is invaluable in planning for educational and therapeutic interventions.

\section{L-04}

\section{The death of a Jamaican prime minister in Canada}

J Stewart*, (Montreal, QC)

Background: Many politicians have died from cerebrovascular disease, changing the course of history.

Methods: Chart review, interviews of physicians, patient relatives.

Results: The Honourable Donald B. Sangster was admitted twice to the Montreal Neurological Institute (MNI). In 1966, aged 54, when acting Prime Minister, he developed diplopia and was referred to the MNI. A 6th nerve palsy was diagnosed and attributed to hypertension. Elections in Jamaica shortly afterward resulted in Sangster becoming Prime Minister. In March 1967 he developed a severe headache. Cerebrospinal fluid was "uniformly bloody"; subarachnoid hemorrhage was diagnosed. He flew to Montreal and was admitted to the MNH. He was drowsy with no focal signs. Carotid angiography showed vasospasm but no source of hemorrhage; ventriculography showed nonspecific abnormalities. He deteriorated: raised intracranial pressure required a ventricular drain, hypertension was treated aggressively. He died on 11th April. Autopsy showed a left anterior communicating aneurysm with adjacent clot, and widespread brain abnormalities from protracted raised intracranial pressure and poor arterial perfusion. Shortly before death Sangster was knighted, although no ceremony was held at the MNI.

Conclusions: Rumours were rife in Jamaica that Sir Donald died from foul play, probably poisoning by the opposition party. This paper establishes the medical facts.

\section{L-05}

Epidemiology of cerebrovascular diseases among ChineseCanadians - a nine-year study of hospitalized patients

$J Y C h u^{*}, J V T u, J K C h u, A G$ Chung, (Toronto, ON)

Background: A pilot retrospective cohort study of the stroke characteristics of hospitalized patients of Chinese descent compared to the non-Chinese population.

Methods: Patients who were admitted through the emergency department with a diagnosis of stroke and discharged with a "most responsible diagnosis" of stroke or CVA between April 1,1990 to March 31,1998 at Trillium Health Centre and Toronto Western Hospital were eligible for the study.

Results: There were 343 stroke patients in the study sample: 142 Chinese, 201 non-Chinese. There was no significant difference in the mean age and sex between the two groups. The frequency of intracerebral hemorrhage was higher among the Chinese patients $34.6 \%$ vs. $23.2 \%,(p=0.023)$. Hypertension was present in $63.5 \%$ of Chinese and in $52.6 \%$ of non-Chinese $(\mathrm{p}=0.044)$. The frequency of smoking, obesity and peripheral vascular diseases were much lower among the Chinese patients while it was higher for valvular heart disease. There was no significant association between in-hospital mortality and ethnic group, stroke location, prior history of hypertension, peripheral vascular disease and obesity.

Conclusions: Important stroke risk factors in Chinese-Canadians were identified through this study. In particular there was a strong association between hypertension and intracerebral hemorrhage in this population. Future long-term studies on differences in genetics, life-style and dietary factors in this population will be vital in understanding these ethnic differences in stroke risk factors and outcomes.

\section{L-06}

\section{Effect of estrogen on functional EREs in mouse ChAT and VAChT 5' regulatory region}

\section{Zerrouki Rachid*, (Montreal, QC)}

Estrogen (E2) is beneficial and long-term deprivation is deleterious to a number of neurotransmitter systems. This has recently become a central issue in Alzheimer disease (AD).

Our study here is based on the hypothesis that: (1) E2 acting via ER-alpha and ER-beta may maintain an adequate expression of ChAT and VAChT genes. (2) There are EREs on the ChAT and VAChT genes that could be regulatory elements for ACh synthesis. 
Our results indicate that in the presence of the hormone (E2), the COS-7 cells express both ChAT and VAChT genes in term of relative Luciferase Unit (RLU). But the Luciferase activity is higher with E2 than without this hormone, which is the basal level. This activity is significantly higher (449\%) with ER-alpha than with ERbeta for ChAT gene, and significantly much higher (2763\%) for VAChT gene. Also in the presence of E2, the activity of ChAT gene is significantly higher than VAChTgene in the presence of ER-alpha $(369 \%)$ or ER-beta (2273\%).

This results show that: (1) E2 maintained an adequate expression of ChAT and VAChT genes and that (2) EREs regulated ChAT and VAChT genes via ER-alpha and ER-beta.

\section{L-07}

\section{Morvan's syndrome associated with biopsy-proven thyroiditis}

\section{A Gagnon*, J De Léan, S Verreault, L Verret (Quebec City, QC)}

Background: Morvan's syndrome is a rare disorder characterized by a triad of neuromyotonia, hyperhydrosis and encephalopathy. Other reported symptoms are pain, weight loss, insomnia and hallucinations. This syndrome has been described in association with thymoma, carcinoma, autoimmune diseases, and autoantibodies, mostly voltage-gated $\mathrm{K}+$ channel antibodies. Symptomatic improvement with plasmapheresis provides further grounds for autoimmune basis. We are reporting, to the best of our knowledge, the first case of Morvan's syndrome along with biopsyproven thyroiditis.

Case report: A 53-year-old man, without any pertinent medical history, developed recurrent stuporous episodes. Subsequently, he presented bilateral orbicularis oculi cramps, diplopia, ataxia, leg weakness, itching, hyperhydrosis, hypersialorrhea, dysphagia as well as both auditory and visual hallucinations. He then progressively developed generalized muscle stiffness with hyperreflexia. EMG revealed abundant neuromyotonic discharges, mostly in the frontalis and axial muscles, which improved further to plasma exchange. Extensive laboratory and radiology investigations revealed positive anti-microsomal and anti-thyroglobulin antibodies with a normal TSH and T4. Thyroid biopsy showed a lymphocytic infiltration characteristic of Hashimoto's thyroiditis. Antibodies to voltage-gated potassium channels were negative. The patient improved remarkably well after plasmapheresis and reported further improvement to us at a four month follow-up visit.

Conclusions: Our case provides further support for an autoimmune pathogenesis in Morvan's syndrome even in patients without demonstrable antibodies to voltage-gated potassium channels. The recognition of this syndrome is of the utmost importance because of its possible reversibility with appropriate therapy.

\section{General NeUrosurgery II}

M-01

\section{Diagnosing pituitary tumors in Nova Scotia}

VMehta*, (Edmonton, AB); L Clarke, R Holness, R Brownstone, D Clarke, (Halifax, NS)

Background: Halifax is ideally suited to study unique epidemiological questions. Our aim was to study from our prospective brain tumor database, the time period required to diagnose pituitary tumors.

Methods: We reviewed our pituitary adenomas for 2001 and 2002. Data was collected prospectively on patient symptoms, time to diagnosis, number of visits to a physician, and tumor pathology.

Results: Over a two-year period we had complete data on 49 patients. $90 \%$ of the cases were macroadenomas and $10 \%$ of the cases had radiographic evidence of apoplexy. The median time to diagnosis was 46 weeks. Thirty-seven percent (18/49) of the patients had secreting tumors. Secreting tumors took significantly longer to diagnose (median 152 weeks) than nonsecreting tumors (53 weeks, $\mathrm{p}=0.02$ ). Headaches were present in $62.5 \%$, visual symptoms in $60 \%$ and endocrine symptoms in $60 \%$ of the patients. The median number of visits to a physician before a correct diagnosis was made was five. No significant difference in the number of physician visits among secreting and nonsecreting tumors was detected.

Conclusions: A significant period of time still is required before the correct diagnosis of a pituitary tumor is made. A longer diagnostic time is required for secreting tumors and a median of five physician visits is required.

\section{M-02}

\section{Minimizing blood transfusions in the surgical correction of craniosynostosis}

P Steinbok*, N Heran, T Hicdonmez, D Cochrane, A Price, (Vancouver, BC)

Background: Avoidance of blood transfusions is a goal of craniosynostosis surgery. This study was performed to determine the rate of allogeneic blood transfusion for craniosynostosis surgery since 1996 and identify factors associated with need for transfusion.

Methods: Craniosynostosis operations in children from 1996 to 2002 were reviewed retrospectively.

Results: There were 72 patients, sagittal 39, unicoronal 14, bicoronal 13 and metopic 6. Median age at operation was 7.5 months. Operations comprised extended strip craniectomies for sagittal, and unilateral or bilateral fronto-orbital advancements for coronal and metopic synostoses. Blood transfusion rates were $7.7 \%$ sagittal, $0 \%$ unicoronal, $31 \%$ bicoronal, and 33\% metopic. Logistic regression was done to examine the relationship of need for transfusion to extent of surgery, surgeons, surgery time, pre-op hemoglobin, craniosynostosis type, weight and age. Of these variables, only more extensive surgery was predictive of need for transfusion. Techniques to minimize blood loss included: use of Colorado needle for scalp incision, Midas Rex craniotome and microfibrillar collagen. Postoperative hemoglobin was allowed to fall to $60 \mathrm{~g} / \mathrm{l}$ if the child was stable hemodynamically, before giving blood. There were no cardiovascular or wound healing complications. 
Conclusions: Low transfusion rates were achieved using simple intra-operative techniques and by accepting a low postoperative hemoglobin.

\section{M-03}

\section{Anti-ischemic effects of topiramate in a transient global forebrain ischemia model: a neurochemical, histological, and behavioral evaluation}

S Khan*, S Wright, A Banigesh, H Miyashita, (Saskatoon, SK);

$K$ Todd, A Shuaib, (Edmonton, AB)

Background: Topiramate (TPM) is neuroprotective in focal cerebral ischemia. We analyzed histological, neurochemical and functional data, to assess neuroprotection in a transient global forebrain ischemia (TGFI) model.

Methods: Thirty-two gerbils were randomly assigned to four groups ( $\mathrm{n}=8 /$ group). Groups I and II received $40 \mathrm{mg} / \mathrm{kg}$ TPM intraperitoneally an hour before and after TGFI, respectively. Groups III and IV (controls) received normal saline similarly. For intracerebral microdialysis, a guide cannula was stereotactically implanted in right CA1. For TGFI, carotid arteries were clamped for five minutes. Modified Morris water maze was used for behavioral testing (BT). Four animals/group were tested five times for seven days. After brain harvesting, neurons were counted to assess damage. To analyze data, statistical significance was set at $\mathrm{P}<0.05$.

Results: All animals survived. Difference in neurochemical response between TPM-treated and control groups post-TGFI, was insignificant. All groups demonstrated decreased latencies during the 7-days BT $(\mathrm{p}<0.001)$. Functionally, difference between groups was insignificant. When the agent was given post-TGFI, there was a trend towards decreased mean percentage neuronal damage in TPMtreated group vs controls ( 67.0 vs $71.7 ; \mathrm{p}<0.06)$.

Conclusions: TPM post-TGFI may attenuate neuronal damage. This attenuation did not cause improved functional outcome.

\section{M-04}

\section{The pentose phosphate pathway theory of neuroprotection}

C Gallagher*, R Tyson, G Sutherland, (Calgary, AB)

Background: A previous study from this lab offered some explanation for the neuroprotective effects of hypothermia in relation to the pentose phosphate pathway. Here we use MR spectroscopy to further develop the PPP theory of hypothermic neuroprotection.

Methods: Rat brain temperature was maintained in the ranges of $37.5 \pm 0.2^{\circ} \mathrm{C}$ in the normothermia group and $31.0 \pm 0.2^{\circ} \mathrm{C}$ in the hypothermia group. One of four labeled substrates, ${ }^{13} \mathrm{C}$ glucose, lactate and acetate were administered. At the end of the infusion period, the brains were prepared and MR spectroscopy was performed.

Results: The amount of ${ }^{13} \mathrm{C}$ label that was incorporated into the TCA metabolites glutamine and glutamate at the $\mathrm{C} 4$ position after infusion of $\left[{ }^{1-13} \mathrm{C}\right]$ glucose was calculated in both conditions. A significant amount of label is not accounted for under the hypothermic conditions when the label is in the $\mathrm{C} 1$ position. Using $\mathrm{C} 2$ label the fractional enrichment was similar. This indicates that no label was lost under hypothermic conditions with $\mathrm{C} 2$ label.

Conclusions: These findings are consistent with an upregulation of the PPP. This could lead to the development of safe compounds to increase the PPP and provide neuroprotection without the adverse effects of hypothermia.

\section{M-05}

\section{Modifiable clinical factors associated with vasospasm: a case-} control study

A Singhal*, R Moulton, P Porter, M Schwartz, A Baker, (Toronto, $O N)$

Background: Previous studies have demonstrated the importance of age, Fisher grade, and clinical grade, in determining the outcome from vasospasm after aneurysmal subarachnoid hemorrhage. It is less clear the impact of modifiable clinical factors such as mean arterial pressure (MAP), central venous pressure (CVP), and hematocrit, on the incidence and outcome of clinical and radiographic vasospasm.

Methods: This study is a case-control analysis that examines clinically-modifiable factors, when age, Fisher grade, and clinical grade are controlled. 80 patients with clinical and radiographic vasospasm were compared to age-, sex-, Fisher grade-, and WFNS clinical grade-matched controls.

Results: Univariate analysis demonstrated that mean change in MAP, mean CVP, fluid balance, and hematocrit were significantly different between vasospasm and control patient groups. Multivariate analysis revealed that these same four factors predicted radiographic vasospasm, and changes in MAP and mean CVP predicted symptomatic vasospasm. None of these factors predicted outcome, as defined by Glasgow Outcome Scores.

Conclusions: Clinically modifiable risk factors can predict the occurrence of radiographic vasospasm and, to a lesser degree, symptomatic vasospasm. This might provide guidance in the initial fluid management after aneurysmal subarachnoid hemorrhage.

\section{M-06}

Repeat imaging just priorto surgery can reduce the rate of inappropriate carotid endarterectomy procedures

J Wells*, S Crocker, S Ilton, (Hamilton, ON)

Background: High grade carotid stenosis can progress to complete occlusion without producing additional symptoms. Carotid endarterectomy after acute asymptomatic internal carotid occlusion is probably contraindicated.

Methods: All carotid endarterectomy cases from a single neurosurgeon's practice during the last two years were reviewed retrospectively. Operative findings were compared with preoperative imaging results and analyzed.

Results: Of the total 122 cases five showed asymptomatic acute total occlusion of the internal carotid artery at surgery. In 31 recent cases we have repeated imaging by Doppler ultrasound within three days of planned carotid endarterectomy. In this group no acute intraoperative occlusions have been encountered. We have cancelled two planned operations when these repeat imaging studies demonstrated complete carotid occlusion.

Conclusions: Routine performance of a carotid Doppler ultrasound within three days prior to planned carotid endarterectomy may significantly reduce the rate of performance of inappropriate operations for patients who experience asymptomatic carotid 
occlusion just before planned carotid endarterectomy. We need more numbers in order to know whether or not pre-operative imaging is statistically significant in preventing unintended surgical exposure of completely occluded carotid arteries.

\section{M-07}

\section{Role of angiopoietins in astrocytoma angiogenesis}

G Zadeh*, B Qian, N Sabha, P Shannon, A Guha, (Toronto, ON)

Background: Angiopoietins (Ang1 \& Ang2) and Tie2 receptor are endothelial cell specific angiogenic factors that are crucial for embryonal vessel development, however, their role in astrocytoma angiogenesis has not been established and is the focus of our study.

Methods: The expression profile of angiopoietins-Tie2 across increasing malignancy grades of astrocytomas was established using Northern and Western blot analysis plus immunohistochemistry on human tumor samples. Angiopoietins were over-expressed in astrocytoma cell lines and grown as subcutaneous and intracranial xenografts in NODSCID mice and analyzed for their growth and vascularity. Tie2 was inhibited using a soluble protein containing only the extracellular portion of Tie2, ExTek. The effect of ExTek on astrocytoma growth and vascularity was assessed in both subcutaneous and intracranial xenografts.

Results: An increasing level of angiopoietin-Tie2 expression and increased Tie2 activation with increasing malignancy grade of astrocytomas is seen. Ang1 enhances tumor vascularity and growth while Ang2 leads to abnormal tumor vascularity. Tie2 inhibition restricts tumor growth and increases survival by $\sim 30 \%$ by disruption of tumor angiogenesis, generating dilated abnormal vessels.

Conclusions: We have shown that the angiopoietins-Tie2 pathway contributes significantly to the vascular growth of astrocytomas and hence can act as a potential therapeutic target for these presently highly malignant tumors. 


\section{Poster Presentations}

\section{Basic Neurosciences}

\section{P-001}

Hsp27 expression during stimulated axonogenesis in dopaminergic neurons of the substantia nigra pars compacta in the adult rat

MO Hebb, EM Denovan-Wright, AM Lozano (Toronto, ON)

Background: Heat shock protein 27 (Hsp27) facilitates regeneration of peripheral neurons. It is not known whether Hsp27 plays an analogous role in the CNS. The purpose of this study was to create an in vivo model in which to assess Hsp27 expression during axonogenesis in the adult brain.

Methods: Autologous nerve grafts were centrally implanted to stimulate axonal growth in dopamine neurons of the rat substantia nigra pars compacta (SNC). Regenerating neurons were identified by retrograde tracer uptake from the graft and assessed for Hsp27 and tyrosine hydroxylase expression.

Results: Axonogenesis was induced in SNC neurons and those adjacent the graft site. Robust Hsp27 induction occurred in regenerating neuronal soma and axons within the graft vicinity, but not distally in the dopaminergic tract or in the SNC. In the latter, $66.5 \%$ (252 of 379 ) of regenerating cells expressed tyrosine hydroxylase immunoreactivity and most exhibited baseline Hsp27 levels. Co-expression of Hsp27 and tyrosine hydroxylase was demonstrated in regenerated dopaminergic axons, and nondopaminergic, Hsp27-positive fibres infiltrated the graft from alternate regions.

Conclusions: Dopaminergic neurons in the substantia nigra of adult animals maintain the ability to regenerate their lesioned axons. Axonal regrowth is associated with the expression of Hsp27. These data provide a novel account of Hsp27 induction in regenerating CNS neurons and, together with its recognized neuroprotective and structural activity, warrant further investigations into the role of Hsp27 in disorders affecting human dopaminergic neurons.

\section{P-002}

C2-3 paravertebral nerve blocks in the treatment of cervicogenic headache

JG Ashby*, (Toronto, ON)

Background: Anatomically the second cervical vertebra is unique and plays a major role in cervicogenic headache. The trigeminal nerve pathways are principal mediators of headache; migraine, cervicogenic headache, tension headache and most other headaches. The descending tract of the trigeminal extends to a varying level in the cervical cord, and with paravertebral or zygapophyseal facet blocks at the C2-3 level, headache in the trigeminal distribution is relieved for a period of several days, i.e. longer than the duration of the nerve block.

Methods: A series of patients with periorbital and frontotemporal headache were treated with C2-3 paravertebral nerve blocks, using $0.5 \%$ Marcaine (bupivacaine hydrochloride) and the duration and extent of headache relief assessed.
Results: The patient's headache, as well as neck pain, were relieved for one to four days, on average, and many reported that this was the only period which they had been pain-free in months. These treatments lessened their dependence on narcotics, decreased their need for emergency visits to hospital and generally improved their quality of life.

Conclusions: C2-3 paravertebral nerve blocks provide effective relief of generalised and retro-orbital headache, thus decreasing dependence on narcotics.

\section{P-003}

Caffeine, nicotine, alcohol and chocolate as they relate to chronic pain

JG Ashby*, (Toronto, ON)

Background: Little has been written about the effects of daily "self-medication" with these four substances that are widespread in use and affect the management of pain.

Methods: A series of chronic pain patients, mainly headache, neck pain and back pain sufferers, have been assessed to better understand the perception of pain, in respect to these four substances.

Results: The majority of the patients learned to better manage their pain, by better understanding their own pathophysiology as affected by the every day use of caffeine, nicotine, alcohol and chocolate, especially those with headache.

Conclusion: Further attention to patients' everyday lifestyle choices can significantly affect pain management.

\section{P-004}

Lipid peroxidation levels are reduced in heat shocked rats suffering acute spinal cord injury

S Christie*, W Currie, I Mendez, (Halifax, NS); B Comeau, (Toronto, $\mathrm{ON}$ )

Background: One of the hallmarks of secondary injury is the ongoing oxidative stress that leads to lipid peroxidation. Heat shock has been shown in various models of injury to minimize the effects of oxidative stress. The objective of this project was to assess the effect of heat shock on lipid peroxidation in a rat spinal cord injury model.

Methods: Fifty female Wistar rats were used. Twenty-five rats were heated $\left(40^{\circ} \mathrm{C}\right.$ for 15 minutes) prior to lesion. Five animals from each group served as controls. The remaining animals received a mid-thoracic complete spinal cord injury via clip compression and were randomly assigned to a sacrifice time, either $4,6,12$, or 24 hours post lesion. Spinal cord tissue was either processed immunohistochemically for hsp27 or assayed colorimetrically for malondialdehyde (MDA) as a marker for lipid peroxidation.

Results: Immunohistochemistry revealed increased expression of hsp27 in the neurons of both the heat-shock control and lesion-only animals. Animals that received a lesion after heat-shock displayed increased expression in both neurons and glia. MDA levels were 
reduced by 35 and $70 \%$ in heat-shocked animals at four and six hours, respectively.

Conclusions: We have shown that heat-shock prior to spinal cord injury induces hsp27 expression in both neurons and glia and leads to a reduction in the degree of secondary injury as measured by lipid peroxidation. These results may have implications in the understanding of secondary injury mechanisms and clinical strategies for spinal cord injury treatment.

\section{P-005}

13C-Labeled substrates and the cerebral metabolic compartmentalization of acetate and lactate

\section{$R$ Tyson*, C Gallagher, G Sutherland, (Calgary, AB)}

Background: Glucose is the predominant energy-producing substrate for brain and, as such, has been the primary substrate used in the study of cerebral metabolism. It has been known for some time that acetate is predominantly utilized in the glial metabolic pool and lactate has recently been shown to be metabolized by neurons. We propose that the use of 13C-labeled lactate and acetate can be used to separate these two metabolic compartments, yielding information not obtainable with glucose alone.

Methods: [1-13C]Glucose, [2-13C]acetate and [3-13C]lactate were infused into male Sprague-Dawley rats (150-170 g) for periods of 3-100 $\min (\mathrm{n}=5$ per time) and after which metabolism was arrested using freeze funnel fixation. Frozen neocortical tissue was extracted using perchloric acid and 13C labeling of water-soluble metabolites analyzed using $13 \mathrm{C}$-edited $1 \mathrm{H}$ magnetic resonance spectroscopy. Blood samples were taken every $15 \mathrm{~min}$ in order to determine the level of labeled substrates in plasma using $1 \mathrm{H}$ magnetic resonance spectroscopy.

Results: The time dependence of the [4-13C]glutamine/[413C]glutamate labeling ratio was significantly different for all three substrates infused $(\mathrm{p}<0.001)$ and showed that acetate is primarily utilized by glia and lactate by neurons, whereas glucose is ubiquitous. The ratio of second- to first-turn TCAcycle labeling for glutamine was significantly lower for acetate (30-100 min infusion; $\mathrm{p}<0.02)$ and greater for lactate $(10-30 \mathrm{~min} ; \mathrm{p}<0.02)$ than for glucose infusions, while the C-2/C-4 glutamate labeling ratio was similar for all the three substrates. This indicated that transfer of [213C] acetate-derived [4-13C]glutamine to neurons rather was preferred to reentry of label into the glial TCA cycle and that the neuronal TCAcycle turnover is significantly faster than that for glia. Fitting parameters of a function representing a pseudofirst order process to the time dependence of labeling demonstrated that GABA labeling reaches steady-state faster with glutamine labeled from [213C]acetate than with glutamate labeled from [3-13C]lactate.

Conclusions: It is concluded that lactate represents a significant improvement over glucose in the study of neuronal metabolism and complements the use of acetate to study glial metabolism and glial/neuronal metabolic relationships.

\section{P-006}

Neural transplantation in the rat model of Parkinson's disease using stem cells cultured in suspension bioreactors

M McLeod*, D Sadi, I Mendez, (Halifax, NS); A Sen, M Kallos, L Behie, (Calgary, $A B$ )

Background: Parkinson's disease (PD) is a neurological disorder caused by the degeneration of dopamine-producing cells in the substantia nigra. Neurosurgical procedures such as neural transplantation utilising stem cells cultured in suspension bioreactors may provide a reliable and reproducible expansion protocol that has the potential to be important therapeutically in the treatment of PD.

Methods: The aim was to investigate the ability of transplanted stem cells produced in bioreactors to survive and integrate into multiple dopamine-depleted brain structures caused by neurochemical lesioning within a rodent model of PD. Cellular morphology and survival of transplanted stem cells was analysed using immunohistochemical techniques.

Results: Immunohistochemical analysis using the neuroepithelial stem cell marker nestin revealed transplanted stem cells survived and integrated into the host tissue. Furthermore, the neuroepithelial stem cells were shown to downregulate nestin expression after eight weeks and differentiate into GFAPexpressing astrocytes.

Conclusion: In conclusion, these findings suggest that transplanted stem cells produced in bioreactors have the potential to differentiate and integrate into host tissue. Therefore, the use of suspension bioreactors for the production of stem cells offers a reliable expansion protocol that has the potential application for future stem cell based restorative therapy in the treatment of PD.

\section{P-007}

Formation of neurons from pluripotent stem cells isolated from a primate muscle

ME Ducharme*, J Rousseau, S Quenneville, JP Tremblay, (Quebec, QC); L Behie, (Calgary, $A B$ )

Background: Adult stem cells have been isolated from several tissue sources like bone marrow, nervous system and skin. We report the isolation of stem cells from adult primate muscles and their differentiation in neuronal cells, which is an excellent alternative source of cells for a therapeutic approach for neurodegenerative diseases.

Methods: Muscle-derived stem cells (MDSCs) were obtained from adult primate muscle biopsies. Following an enzymatic dissociation of the tissue, spheres of cells were obtained after 7-10 days in a proliferation medium. Cells were plated on coated poly-Dlysine/gelatin petri dishes in a neuronal differentiation medium for 12 days. The differentiated cells were characterized by immunostaining for two neuronal markers (beta-III tubuline and neurofilament M), for an astrocyte and Schwann cells marker (glial fibrillary acidic protein, GFAP) and for a myoblast marker (desmin).

Results: Between $1 \%$ and $5 \%$ of differentiated cells were neurons, as observed by specific labelling of the two neuronal markers. Moreover, all differentiated neurons were negative for desmin. Interestingly, no astrocyte or Schwann cells were observed in our differentiated cultures. 
Conclusions: The use of these MDSCs to produce neuronal cells for autologous treatment of patients with neurological diseases would permit to avoid a graft rejection problem.

\section{Cerebrovascular Diseases}

\section{P-008}

Bilateral ischemic anterior temporal opercular syndrome with inverse automatic-voluntary dissociation

V Sim*, A Guberman, M Hogan, (Ottawa, ON)

Background: The acquired anterior opercular syndrome (AOS) usually involves automatic-voluntary dissociation of facial movements. In most cases automatic movements are selectively preserved but the inverse can also occur. Bilateral sequential strokes are the most common cause, but bilateral acute infarcts can occur. Patients with bilateral acute infarcts are more likely to have unusual risk factors, therefore recognition of this rare syndrome is important.

Case report: A case of acute bilateral AOS with inverse dissociation, impaired swallowing and abnormal hearing recognition in a 50-year-old, previously healthy woman, is presented. There was restricted MRI diffusion in both temporal opercula, right frontal gray matter, and right centrum semiovale. The diffusion abnormalities and clinical symptoms resolved within five days following treatment with heparin.

Conclusions: 1) Inverse dissociation is a recognizable syndrome of bilateral acute AOS. 2) Voluntary and involuntary pathways are distinct. Involuntary pathways may be more susceptible to ischemia. 3) Restricted diffusion on MRI does not always represent irreversible cytotoxic damage.

\section{P-009}

Outcome in acute MCAand basilar artery stroke treated with intra-arterial tPA and balloon angioplasty - the Ottawa experience

C Lum*, P Stys, W Miller, M Hogan, M Sharma, M Goyal, (Ottawa, ON)

Background: Thrombolysis with intra-arterial tPA (ia-tPA) has shown benefit in patients presenting with acute MCAterritory stroke within six hours and in basilar artery (BA) thrombosis. Small series have described balloon angioplasty of acute MCA clot. This study reviews our outcomes in patients presenting with acute MCA/BA thrombosis treated with both ia-tPA and/or clot angioplasty and compares the MCA data to the PROACT II trial (40\% good outcome, $10 \%$ bleeds, $25 \%$ mortality).

Methods: Retrospective analyses of charts, images and telephone interviews were conducted for acute stroke patients treated in our interventional neuroradiology suite between April 2001-Dec 2002. Good clinical outcomes assessed using the modified Rankin scale (mRS) and Barthel Index (BI) corresponded to values of less than or equal to 2 and $>90$, respectively. Intracerebral bleeds resulting in neurological deterioration and deaths were noted.

Results: Twelve patients had proximal MCA clot. Nine of these (75\%) had good outcomes of which three had successful balloon angioplasty of tPA refractory clot and one had angioplasty alone. Intracerebral hemorrhage occurred in one ( $8 \%$ ), given tPA only, who subsequently died. One of seven patients with basilar thrombosis had a good outcome; five of seven $(71 \%)$ died.

Conclusions: In this small series, the percentage of patients with MCAstroke who had good outcomes after recanalization therapy is comparable to PROACT II. Complication rates are similar to PROACT II. Balloon angioplasty is an important technique to be considered in tPA refractory clot but requires further investigation. Outcome for patients with BA thrombosis is dismal despite technologies such as ia-tPA and angioplasty.

\section{P-010}

Giant posterior cerebral aneurysm following interventional coiling: a case report

RT Grondin*, R Ashforth, DE Steinke, (Edmonton, AB)

Background: The sequelae of intracranial aneurysm remnants after interventional placement of Guglielmi detachable coils (GDC) are not well established.

Methods: We report the case of a 46-year-old woman.

Results: She initially presented with a subarachnoid hemorrhage from a P1/P2 junction posterior cerebral artery aneurysm and underwent interventional coiling with Guglielmi detachable coils. A small neck remnant persisted. She showed minimal neurologic deficit after rehabilitation. Eighteen months later, she presented with increased intracranial pressure. Computed tomography of the brain demonstrated noncommunicating hydrocephalus secondary to a giant aneurysm compressing the third ventricle. Cerebral angiography showed partial thrombosis and slow filling of the giant aneurysm that had arisen from the residual neck. The aneurysm was trapped with Guglielmi detachable coils placed proximal and distal to the aneurysm neck. The noncommunicating hydrocephalus was treated with a ventriculo-peritoneal shunt. Despite a favourable outcome, she was left with significant neurologic deficit.

Conclusions: We propose that residual necks from GDC-coiled aneurysms are at risk of expanding and/or re-bleeding causing significant adverse neurologic sequelae. Patients with neck remnants should be followed by angiography and/or magnetic resonance angiography. A prospective study is needed to assess this risk.

\section{Cerebrovascular Surgery}

\section{P-011}

\section{Hyperperfusion syndrome - more than one disease?}

$S$ Coutts*, W Hu, G Sutherland, M Hill, (Calgary, AB)

Background: Hyperperfusion syndrome (HS) is a rare and potentially devastating complication of internal carotid endarterectomy (CEA) or carotid angioplasty and stenting (CAS). With the advent of new imaging techniques we sought to review our experience of this disease and to explore alternative explanations for the cases.

Methods: We performed a retrospective review of 129 consecutive CEAcases over the period from 1st June 2000 until 31st May 2002 and 44 consecutive cases of CAS from 1st January 1997 until 31st May 2002. We searched specifically for examples of HS. In all patients, the charts and imaging were reviewed.

Results: Six cases of HS occurred (three post CEA(2.33\%) and 
three post CAS (6.8\%)). The cases of hyperperfusion can be divided as follows: 1) Acute focal edema - two cases of a stroke like presentation, due to edema immediately post-CEAor CAS. 2) Acute hemorrhage - two cases of intracerebral hemorrhage (ICH) immediately post CAS. 3) Delayed (after 24 hours) classical presentation - two examples of seizures, focal motor weakness, and/or late ICH post CEA.

Conclusions: Hyperperfusion syndrome after carotid revascularization likely represents several distinct diseases with different pathophysiologies.

\section{P-012}

\section{An atypical case of a posterior fossa arteriovenous} malformation presenting with hydrocephalus

\section{Rabin*, J Spears, MC Wallace, (Toronto, ON)}

Background: Approximately 12\% of arteriovenous malformations (AVM) arise infratentorially and 79\% of these lesions present with intracranial hemorrhage (ICH). We describe the case of a 22year-old male with a large posterior fossa AVM occupying the majority of the left cerebellar hemisphere and causing compression of the 4th ventricle with secondary hydrocephalus (HC). No evidence of recent or remote ICH was observed. Urgent ventriculoperitoneal shunting and serial embolization of the AVM resulted in clinical improvement.

Methods and Results: A review of the literature identified four potential mechanisms underlying the relationship between cerebrovascular malformations and $\mathrm{HC}$. These include $\mathrm{HC}$ exvacuo, $\mathrm{HC}$ secondary to $\mathrm{ICH}, \mathrm{HC}$ secondary to venous hypertension and noncommunicating HC. Five accounts of posterior fossa AVM presenting with symptoms, signs and imaging consistent with $\mathrm{HC}$ in the absence of clinical or radiological evidence of ICH are reported in the literature. Four of five cases demonstrated some clinical improvement with either CSF shunting or AVM excision or embolization. Optimal improvement was observed with a combination of these techniques in all four individuals. The remaining case demonstrated marked clinical deterioration with CSF shunting.

Conclusions: Optimal management of HC secondary to posterior fossa AVM likely requires CSF diversion and AVM embolization or excision. Both CSF obstruction and venous hypertension may contribute to the development of $\mathrm{HC}$ in these uncommon lesions.

\section{P-013}

\section{Delayed cerebral vasospasm after surgical clipping of an unruptured intracranial aneurysm: case report and literature review}

\section{A Mitha*, J Wong, (Calgary, AB)}

Background: Delayed cerebral vasospasm represents a significant complication in the course of subarachnoid hemorrhage caused by intracranial aneurysms. The exact mechanism of vasospasm remains unclear. Current etiological theories, however, are based upon the presence of blood in the subarachnoid space.

Methods: We present a case of symptomatic delayed cerebral vasospasm after surgical clipping of an unruptured aneurysm. A literature search was done to find documentation of similar cases. Details of these cases, guided by proposed pathophysiological mechanisms, are reviewed and compared.
Results: Vasospasm associated with unruptured aneurysms is extremely rare. A disproportionate number occurred in female patients. Occasionally, angiographic vasospasm has been demonstrated prior to surgical intervention of aneurysms without evidence of subarachnoid hemorrhage. Cerebral vasospasm following surgical intervention of unruptured aneurysms can be divided into early and delayed onset, suggesting different pathophysiologic mechanisms.

Conclusions: Cerebral vasospasm is widely regarded as a complication following subarachnoid hemorrhage. This case report and literature review, however, reveals that subarachnoid blood is not necessary for clinically significant vasospasm to occur. Until the exact mechanisms are elucidated, it is important to think about cerebral vasospasm in terms of its potential pathophysiological origin rather than attributing it to aneurysmal subarachnoid hemorrhage alone.

\section{P-014}

\section{Predictors of severity of subarachnoid hemorrhage}

S Christie*, A Asyyed, K Nichol, T Christie, I Fleetwood, (Halifax, $N S)$

Introduction: Survival and outcome following subarachnoid hemorrhage (SAH) has been linked to clinical status at the time of the ictus. The objective of this study was to identify risk factors associated with the initial severity of SAH.

Methods: All patients with aneurysmal SAH at our centre between 1999 and 2001, inclusive, were identified prospectively. A chart review was conducted to complete demographic and risk factor data. Risk factors assessed included age, sex, smoking status, location, size of aneurysm, multiple aneurysms, family history of aneurysms, history of hypertension, diabetes, cardiac disease, previous stroke and alcohol use. A backwise logistic regression was used to predict the odds of having a severe hemorrhage (Hunt and Hess grade IV or V) versus a moderate hemorrhage (Grade I-III).

Results: Twenty of 132 patients were excluded due to incomplete data. Of 112 patients analyzed the mean age was $55.54 \pm 13.1$ years, $82(73.2 \%)$ were women, $84(75.0 \%)$ were smokers and $12(10.7 \%)$ had a positive family history. There were 89 (79.5\%) moderate hemorrhages and $23(20.5 \%)$ severe hemorrhages. Patients who smoke or had a positive family history were more likely to have a severe hemorrhage (OR 1.64; 95\% CI 1.02-1.87 and OR 1.95; 95\% CI 1.73-1.99, respectively).

Conclusions: Our results suggest that aneurysm patients who smoke or have a positive family history are at greater risk for severe SAH.

\section{P-015}

Incidence of pulmonary edema and cardiac dysfunction following subarachnoid hemorrhage

N Mc Laughlin*, MW Bojanowski, F Girard, A Denault, (Montreal, $Q C)$

Introduction: Cardiogenic component in pulmonary edema (PE) following subarachnoid hemorrhage (SAH) remains incompletely understood. We describe the incidence of early and delayed PE and reveal the existence of myocardial dysfunction (MD) suggesting a cardiogenic contribution. 
Methods: Retrospective review of patients with SAH from 041998 to 04-2001. Patients having PE on chest radiographs were included. MD was assessed by standard hemodynamic criteria. MD criteria were: central venous pressure and pulmonary capillary wedge pressure above $15 \mathrm{mmHg}$, mean pulmonary arterial pressure (MPAP) above $25 \mathrm{mmHg}$, ratio mean arterial pressure (MAP) on MPAP less than 4, expiratory end tidal $\mathrm{CO}_{2}$ less than $25 \mathrm{mmHg}$, cardiac index less than $2,5 \mathrm{~L} / \mathrm{min} / \mathrm{m}^{2}$, abnormal systolic and diastolic function on echocardiography.

Results: Of 178 cases of SAH following ruptured aneurysms, 42 cases were included $(23,6 \%)$ from which $47 \mathrm{PE}$ episodes were identified. Five episodes had an early onset $(10,6 \%)$ and 42 a delayed onset $(89,4 \%)$. Intra-operatively, 43 episodes satisfied at least one abnormal hemodynamic criterion $(91,5 \%), 24$ satisfied at least two criteria $(51,1 \%)$. MD was found in $40 \%$ of echocardiographies performed.

Conclusions: Majority of PE have a delayed onset and a cardiogenic component. Recognition of systolic and/or diastolic dysfunction could have therapeutic implications.

\section{P-016}

Early surgery in patients with documented systolic and diastolic dysfunction following subarachnoid hemorrhage: case series

\section{N Mc Laughlin*, MW Bojanowski, A Denault, (Montreal, QC)}

Introduction: Systolic myocardial dysfunction (MD) may contribute to pulmonary edema (PE) following subarachnoid hemorrhage $(\mathrm{SAH})$. We have proposed that diastolic myocardial dysfunction (DMD) may also be a contributing factor. Timing for surgery in patients with SAH presenting MD and PE is unclear. This new notion and its therapeutic implications are illustrated by three cases.

Methods: Case series.

Results: Two patients were Hunt \& Hess grade IV and one grade III. All arrived at our hospital hemodynamically unstable and required vasoactive drugs. Initial echocardiographic study done preoperatively documented moderate to severe left ventricular (LV) systolic MD. LV diastolic MD was diagnosed using accepted criteria (Redfield. JAMA 2003; 289: 194-202). Patients underwent early surgery. Follow-up echocardiographic exams revealed improvement of MD. All patients achieved good outcome prior to discharge.

Conclusions: Systolic and diastolic MD may contribute to hemodynamic instability and pulmonary edema following SAH. Early surgery is not necessarily a contra-indication in this setting.

\section{P-017}

\section{The endovascular management of superior cerebellar artery aneurysms}

C Haw*, K ter Brugge, $R$ Willinsky, (Toronto, ON)

Background: Superior cerebellar artery aneurysms are rare. We present a clinical series of twelve of these aneurysms that were treated exclusively with endovascular coils.

Methods: A retrospective analysis of a prospectively collected database of cerebral aneurysms treated with coil embolization was performed. Clinical notes and radiological images were reviewed.

Results: Twelve superior cerebellar artery aneurysms were treated in eleven patients between 1992 and 2001. Seven patients presented with subarachnoid hemorrhage, two with neurologic deficit, and two had asymptomatic aneurysms. Coiling resulted in complete aneurysm obliteration in six patients and incomplete obliteration in the other six. No subsequent hemorrhage occurred with follow-up between six and 119 months (mean follow-up 45 months). Procedural morbidity was one superior cerebellar artery infarct with good recovery. Management morbidity was one middle cerebral artery embolus during a follow-up angiogram that required thrombolysis with a good clinical result. Nine out of eleven patients on follow-up were performing at Glasgow Outcome Scale (GOS) 5. One patient with GOS 3 presented with a poor grade subarachnoid hemorrhage and the other patient with GOS 4 presented with a parenchymal hemorrhage due to an arteriovenous malformation.

Conclusions: Endovascular treatment of superior cerebellar artery aneurysms is an effective treatment strategy with low morbidity. Even partial aneurysm coiling may be effective in preventing rehemorrhage in long-term follow-up.

\section{Child Neurology}

\section{P-018}

\section{Depression in parents of children with Duchenne muscular dystrophy}

M Abi Daoud, J Dooley*, K Gordon, (Halifax, NS)

Background: In a previous study (Dooley et al, 2002), many parents of boys with Duchenne muscular dystrophy (DMD) indicated that depression was a factor in their lives. The current study was undertaken to more accurately quantify depression in the caretakers of a group of males with DMD in comparison to a control group.

Methods: A questionnaire, based on the National Population Health Survey (NPHS) (Mental Health Section) was conducted with caretakers of children with DMD. The results were compared with 168 matched control participants randomly obtained from the NPHS data set (1994 \& 1999).

Results: 42/60 (70\%) parents, having 27/35 (77\%) DMD boys (average age of 15.6 years) completed questionnaires. DMD parents had a higher probability of going through a major depressive episode $(\mathrm{p}<0.001)$ and had more distress in their lives $(\mathrm{p}<0.001)$. DMD parents were as likely to consult mental health professionals and report similar durations of depressive symptomatology as depressed controls. Self-esteem and mastery scores were significantly lower in parents of children with DMD ( $\mathrm{p}<0.001)$.

Conclusions: Health professionals who care for children with DMD should be aware of the distress experienced by their caretakers and of the likelihood of depression.

\section{P-019}

Successful endovascular therapy for acute basilar thrombosis in an adolescent

A Kirton*, JH Wong, J Mah, BC Ross, J Kennedy, MD Hill, (Calgary, $A B$ )

Background: Pediatric stroke is an under-recognized, potentially treatable cause of childhood neurological disease. Acute basilar 
artery thrombosis is a devastating disease rarely encountered in children. Acute interventions with both chemical and mechanical thrombolysis techniques can improve outcomes in adults with arterial thrombosis of the posterior cerebral circulation.

Methods: Case report.

Results: We report an example of intervention with both intraarterial alteplase (tPA) and cerebral balloon angioplasty to treat a prolonged basilar artery occlusion secondary to idiopathic thrombosis in an adolescent. Despite the patient being clinically locked-in, and intervention being delayed at least 20 hours from symptom onset, he obtained complete neurological recovery. Issues of pediatric stroke, late therapeutic intervention, chemical thrombolysis, and cerebral angioplasty are discussed.

Conclusions: This case highlights the under-recognition, and subsequent delay in diagnosis, of pediatric stroke and how acute intervention may cure otherwise catastrophic strokes in children.

\section{P-020}

\section{Acute necrotizing encephalopathy of childhood in two Caucasian children}

\section{A Kirton*, K Busche, D George, B Ross, E Wirrell, (Calgary, AB)}

Background: Acute necrotizing encephalopathy (ANE) is a recently described, poorly understood and devastating neurological disease of children. It is rarely seen in Caucasian populations.

Methods: Case series.

Results: We present and compare two cases of ANE diagnosed at a Canadian center within the same year in two young Caucasian children. The cases are comparable in that both children were developmentally normal, were previously well aside from simple febrile seizures in one, and had no significant family histories. They then developed an acute viral illness with fever and vomiting and unexpectedly progressed to clinical brain death within 2-4 days. In both cases, neuroimaging and postmortem examination demonstrated the unique features of ANE, namely bilateral and severe necrosis of deep gray and subcortical white matter structures. Distinct features of one case included demonstration of influenza A infection and absence of hepatic involvement. Distinguishing features of the other case included extensive but transient hepatic involvement with necrosis and steatosis on biopsy, varicella infection two weeks previous, and an extensive metabolic work-up, including mitochondrial functional analysis, which was normal. Both cases are distinct for their occurrence in non-Japanese children and are the first two published reports of ANE in Canada.

Conclusions: ANE is a catastrophic neurological disease that can occur in Caucasian Canadian children.

\section{P-021}

Eyelid myoclonia with absences: about two familial pediatric observations

\section{Z Belkadi*, (Laon, France); J Motte, (Reims, France)}

Background: Eyelid myoclonia with absence has been lately described as an epileptic syndrome fully distinct from childhood absence epilepsy.

Methods: We report the case of two children brother and sister presenting, since the age of five and 10 years respectively, with rapid eyelid myoclonias with upward eye deviation, sometimes accom- panied by brief absence and arising at the eyes closure.

Results: Ictal EEG shows a brief 3-5 Hz generalized discharge of polyspike waves. Overbreathing and intermittent photic stimulation increase interictal EEG abnormalities. Psychomotor and intellectual development as well as neurological examination of both children was normal. However, in spite of numerous anti-epileptic drugs, the two children did not become seizure-free.

Conclusions: We add our observations to the previous ones to confirm that eyelid myoclonia with absence differs from childhood absence epilepsy and is a specific epileptic syndrome. The likelihood of a genetic origin of this idiopathic generalized epilepsy is strong.

\section{P-022}

Idiomatic language function and corpus callosum dysmorphology in children with spina bifida

J Huber-Okrainec*, (Hamilton, ON); S Blaser, M Dennis, (Toronto, $\mathrm{ON}$ )

Background: The fully developed corpus callosum (CC) is part of a distributed neural system for non-literal language, such as idioms, phrases for which the figurative meaning cannot be derived from the literal meanings of the individual words. We studied how children with CC dysmorphology understand idioms.

Methods: Participants were 37 children aged 8-18 years, assigned to four age and IQ matched groups: controls $(\mathrm{N}=11)$; spina bifida (SB) and intact $\mathrm{CC}(\mathrm{N}=8)$; $\mathrm{SB}$ and hypoplasia of the $\mathrm{CC}(\mathrm{N}=10)$; and $\mathrm{SB}$ and agenesis of the $\mathrm{CC}(\mathrm{N}=8)$, based on MRI findings. In an idiom comprehension task, participants judged whether a heard familiar idiom matched a picture. Idioms varied in compositionality (nondecomposable vs. decomposable; whether the words contributed to the figurative meaning) and literality (high vs. low literal; whether a literal interpretation was plausible). Accuracy and response time were measured.

Results: Children with SB showed poor idiom comprehension, which varied with idiom type and extent of CC dysmorphology. Deficits were greater for individuals with callosal agenesis for nondecomposable or high literal idioms.

Conclusions: Comprehension of idioms varies with CC dysmorphology. Successful idiom comprehension may require varying degrees of integration of information from each cerebral hemisphere, facilitated by an intact CC during development.

\section{P-023}

\section{Effectiveness of melatonin forsleep disturbance in a pediatric neurology clinic}

\section{E Wirrell*, L Hamiwka, M Blackman, (Calgary, AB)}

Background: Sleep disturbances are common in children with neurodevelopmental disorders. Melatonin may be of benefit in this population.

Methods: Retrospective review of a cohort of children treated with melatonin.

Results: 14 children (7M:7F, median age $4.5 \mathrm{yrs})$ were started on melatonin for sleep disturbance between 16/08/00 and 22/10/02. All were developmentally delayed ( 2 mild-moderate, 12 severe), 12 had epilepsy and five had pervasive developmental disorder. Median melatonin dose was $3 \mathrm{mg}$ (range $2-5 \mathrm{mg}$ ), and median duration of 
treatment was six months. Overall, $10(71 \%)$ had significantly improved sleep as rated by their caregivers. The median number of nights per week of disrupted sleep prior to melatonin was 7 (25-75th percentile 5-7) and on melatonin was 2 (0-7). Of the eight patients with initial insomnia, $6(75 \%)$ were improved $90-100 \%$, while two were not helped. Of the 12 with recurrent night awakening, 7 (58\%) were improved more than $75 \%, 2(17 \%)$ were improved $25-50 \%$ and $3(25 \%)$ were unchanged. No child had worsening of seizures. Cognition and concentration were improved in 7 (50\%) while behavior was better in 4 (29\%). No patient suffered adverse effects.

Conclusions: Melatonin appears to be a safe and effective treatment for sleep disturbance in children with neurodevelopmental problems, however its efficacy should be documented in a prospective study.

\section{P-024}

\section{Bilateral frontoparietal polymicrogyria in Pakistani siblings}

$H$ Glass*, E Wirrell, M Innes, (Calgary, $A B$ )

Background: An autosomal recessive form of bilateral frontoparietal polymicrogyria (BFPP) was recently mapped to chromosome 16 (Piao, et al. Am J Hum Gen, 2002) in consanguineous Palestinian pedigrees. Children with the disorder have a congenital syndrome including developmental delay, seizures, esotropia and pyramidal signs. Neuroimaging shows bilateral, symmetric polymicrogyria in frontal and parietal lobes, and patchy white-matter lesions. Genetic and metabolic investigations are normal. Patients lack dysmorphic features and other congenital anomalies.

Methods: We review the presentation of Pakistani siblings whose brain imaging and clinical findings are consistent with BFPP.

Results: A three-year-old Pakistani boy presented with refractory seizures, esotropia and developmental delay. His examination showed decreased tone, muscle mass and reflexes, as well as upgoing plantar responses. His younger sister attended the appointment with him, and was noted to have bilateral esotropia and developmental delay. MRI showed symmetric polymicrogyria with white matter abnormalities in both patients. Metabolic investigations and karyotype were normal. Gene mapping is pending.

Conclusions: Our patients'phenotype is consistent with BFPP, a rare genetic syndrome that can mimic other white matter diseases including metachromatic leukodystrophy, Krabbe and Canavan. The underlying defect is not known and linkage to chromosome 16 is the only means of ascertainment.

\section{P-025}

\section{Genetic epidemiology of pediatric MS: pilot study}

BL Banwell*, (Toronto, ON); CH Guimond, AD Sadovnick, (Vancouver, BC)

Background: The genetic epidemiology for adults with MS followed in Canadian clinics is well-documented and has provided critical insight into the disease. The cohort of the Pediatric MS Clinic at the Hospital for Sick Children is unique as it includes over 35 cases of pediatric MS, with the earliest age of onset being four years.

Methods: Using standard genetic epidemiological techniques, we are collecting data for this population, including a family history, family structure, and ethnicity. These pilot data will be compared to adults who, in retrospect, report an age of MS onset at age 16 or younger.

Results: Preliminary analyses suggest that the pediatric population over represents non-Caucasian ancestry, most of who represent second generation migrants. At least $16 \%$ of pediatric cases have a positive family history and it is imperative to follow their parents who in general are still well within the age range (25$40)$ at risk to develop MS. Thirty-five percent of the pediatric cases have a family history of other autoimmune disorders.

Conclusions: It is expected that this cohort will provide unique insights into the etiologic and phenotypic heterogeneity of MS. We plan to expand the genetic epidemiological database through collaborative efforts within North America and other countries. We are also collecting DNAfrom these families to allow for molecular genetic studies

\section{P-026}

\section{A juvenile case of type IVglycogenosis with cardiomyopathy}

J Mah*, (Calgary, AB); Y Al-Khatib, (Akron, OH); R Hessler, (Augusta, GA)

A previously well nine-year-old boy presented with severe dilated cardiopathy after an upper respiratory tract infection. His neurological exam showed bilateral ptosis and mild proximal muscle weakness. Investigations including thyroid functions, liver enzymes, glucose, creatine kinase, carnitine, lactate, and metabolic screen were normal. Electromyography exam showed myopathic motor units. Subsequent muscle biopsy revealed type I predominance, with marked variation in fiber size, and numerous subsarcolemmal inclusions, which were periodic acid-Schiff positive and diastase resistant. Electron microscopy demonstrated the inclusions to be granular and filamentous in appearance. Biochemical analysis confirmed the diagnosis of glycogen storage disease (GSD) type IV or Andersen disease, with absent branching enzyme activity. While awaiting cardiac transplantation, the patient developed a large intracardiac thrombus and died eight months after his initial presentation. Children with GSD type IV typically present with progressive hepatic failure and death by age five. Juvenile variants of GSD type IV with cardiac or neuromuscular involvement are uncommon. It is important to include this form of GSD in the differential diagnosis of children presenting with cardiopathy or myopathy. As similar polyglucosan inclusions are seen in Lafora disease, the accumulations of abnormal glycogen likely contribute to the pathogenesis of both disorders.

\section{P-027}

\section{Spinal muscular atrophy with respiratory distress type 1 (SMARD1)}

\section{Adams*, (Victoria, BC)}

Background: Spinal muscular atrophy with respiratory distress type 1 (SMARD1) results from mutations in the gene encoding immunoglobulin mu binding protein (IGHMBP2) on chromosome 11q13.2-q13.4 leading to premature termination of protein synthesis. (Grohmann, et al. Nat Genet 2001;29:75-77).

Clinical information: Presented is an infant with SMARD1 (also known as severe infantile axonal neuropathy). Parents were first 
cousins of Lebanese origin. The infant had reduced fetal movement, intra-uterine growth retardation and congenital equinovarus feet. At three months he developed respiratory distress. At six months he had truncal hypotonia, wasting of intrinsic hand and lower leg muscles with distal weakness and absent distal deep tendon reflexes. Diaphragm weakness was more prominent than limb weakness. He was ventilated at seven months and subsequently showed progression of his weakness. Nerve conduction studies showed an axonal sensory-motor neuropathy and sural nerve biopsy showed evidence of axonal degeneration. Molecular genetic testing found no abnormality of the survival motor neuron (SMN) or Charcot-MarieTooth (CMT1a, 1b or X) genes. This infant carried on both his chromosomes the nonsense mutation L236X in exon 5 of the IGHMBP2 gene.

Conclusion: This presentation is to increase awareness of this entity and the availability of prenatal diagnosis.

\section{P-028}

\section{Imaging function and cerebrovascular response in an anesthetized child}

WLogan*, (Toronto, ON)

Background: The utility of MRI to localize brain function and to determine regional cerebrovascular response (CVR) has been well demonstrated. These studies generally require a cooperative subject, but this is not always possible, particularly in children. There are reports of functional imaging in sedated patients using passive stimulation techniques. This study examines imaging of function and CVR in an anesthetized child.

Methods: The subject was a 10-year-old male with intractable seizures secondary to a partial hemimegalencephaly (HM). The imaging was requested to delineate the abnormal brain anatomy and function in relation to the normal brain. A BOLD MRI technique was used, and images were obtained using a 1.5 Tesla scanner. The visual stimulus was a stroboscopic flash, the auditory stimulus was a story and the somatosensory stimulus was brushing of the hands. CVR was examined by varying the respiratory rate and the $\mathrm{pCO}_{2}$. Propofol anesthesia was used for the study.

Results: Each of the sensory stimuli produced focal brain activation and CVR activation was demonstrated. The sites of activation and the CVR were distorted in the region of the HM.

Conclusions: It is possible to perform imaging of sensory function and CVR in an anesthetized child.

\section{P-029}

\section{Radiographic changes in an infant with Menkes disease}

\section{A Ho, J Mah*, R Casey, P Gaul, (Calgary, AB)}

A four-month-old infant boy presented with developmental delay and new onset seizures. He had repeated episodes of staring and unresponsiveness one week prior to admission. Review of past medical history revealed fetal distress requiring brief resuscitation at birth. Physical examination was remarkable for truncal hypotonia and short bristly blond hair. Investigations including serum glucose, electrolytes, calcium, and metabolic screen were normal. Serum and CSF lactate were elevated. The diagnosis of Menkes disease was made by hair exam under light microscopy, and confirmed by low plasma ceruloplasmin and copper levels. Brain MRI study demonstrated cerebral atrophy, with diffuse white matter abnormalities and tortuous vessels. After treatment with subcutaneous copper histidinate, there was noticeable improvement in the appearance of the white matter on follow-up MRI exam. However, the patient continued to have refractory seizures and made no developmental progress. Reduced activity of several copper dependent enzymes, including cytochrome $\mathrm{C}$ oxidase and lysyl oxidase, is responsible for the clinical and the radiological findings in Menkes disease. Testing for mutations within the copper transporting ATPase gene on Xq13.3 is helpful for genetic counseling and determination of response to copper therapy.

\section{P-030}

Tuberous sclerosis and schizencephaly: a case report and review of the literature

\section{$R$ Huntsman *, D Sinclair, L Richer, (Edmonton, AB)}

Background: Recent evidence suggests that the central nervous system lesions in tuberous sclerosis are secondary to a defect in cell maturation and migration. There are case reports of various neuromigrational disorders associated with tuberous sclerosis. Schizencephaly has only once been reported in association with tuberous sclerosis. We present a case of open lip schizencephaly in a child with tuberous sclerosis.

Methods: The patient's hospital chart was reviewed as were all EEG and neuroimaging studies. In addition, the literature was reviewed looking for other reported cases of tuberous sclerosis and schizencephaly.

Results: The child presented prenatally with cerebral and cardiac lesions detected on ultrasound. Postnatal cranial CT and MRI showed findings consistent with tuberous sclerosis and extensive open lip schizencephaly affecting the right fronto-parietal lobe. He later developed West syndrome treated with vigabatrin.

Discussion: There is a spectrum of cerebral dysgenesis associated with tuberous sclerosis. To our knowledge, this is the first report of tuberous sclerosis associated with open lip schizencephaly. The literature suggests that cerebral lesions found in tuberous sclerosis are secondary to a defect in neuronal maturation and migration. Our case is consistent with this hypothesis. Clinicians should be aware of the possibility of cerebral dysgenesis in tuberous sclerosis.

\section{P-031}

\section{Hyperthyroidism and status migrainosus}

M Belletrutti*, M Moodley, N Lowry, (Saskatoon, SK)

Purpose: To heighten awareness of the association between thyroid disorders and migraine in children.

Background: Even though headache is one of the most common complaints of children, the association of headache with thyroid disorders is rare. There are several adult studies showing an association between hypothyroidism and migraine, but very few documenting an association with hyperthyroidism.

Patient: An adolescent female presented with a one day history of severe, right-sided headache associated with nausea, vomiting, photophobia, and right-sided weakness. Previously, she had suffered several episodes of migraine-type headaches, which responded to simple analgesics. Family history is significant for migraine in her 
mother and sister. Clinical examination revealed a well-nourished child with fullness of the anterior neck, a faint bruit over the thyroid gland, and a normal neurological examination.

Results: Basic bloodwork and MRI were normal. Conventional migraine therapy failed to resolve her symptoms. Thyroid function tests showed thyrotoxicosis. Her symptoms resolved only after institution of antithyroid therapy. One-year follow-up shows complete resolution of her symptoms on propylthiouracil.

Conclusions: This is the first documented pediatric case of thyrotoxicosis presenting as status migrainosus and serves to heighten awareness of this association, as early recognition is essential for appropriate investigation and management.

\section{P-032}

\section{Surgical treatment for tentorial herniation in herpes encephalitis: a case report}

\section{Hamiwka*, M Hamilton, M Blackman, (Calgary, AB)}

Background: Herpes simplex is the most common cause of acute viral encephalitis in children. Even when medical therapy is established early, significant edema and tentorial herniation can develop. There is little information regarding surgical treatment with only one case reported in the literature.

Methods: We report on an 11-year-old girl.

Case: She presented with a one-week history of fever, headache, vomiting and no focal neurologic signs. Investigations in the emergency room included a lumbar puncture, which was PCR positive for herpes. Early treatment included IV acyclovir. Her state of alertness initially improved, however 24 hours later, she became obtunded with a left hemiparesis and bilateral 6th nerve palsies. Magnetic resonance imaging showed swelling of the right temporal lobe and compression of the cerebral peduncle. She underwent a right temporal lobectomy. Postoperatively she remained comatose for 14 days. The early recovery showed a significant left hemiparesis, difficulty swallowing and no speech. After rehabilitation she has showed a remarkable recovery with mild cognitive difficulties and left hemiparesis.

Conclusions: Our case represents good outcome with minimal neurologic deficit. We suggest that temporal lobe resection and decompression craniotomy may be beneficial in cases with tentorial herniation to prevent secondary brain injury and potentially reduce infectious material.

\section{P-033}

\section{Intracranial aneurysm: a rare cause of cerebral infarction in childhood}

\section{Huh*,M Sargent, A Price, E Roland, (Vancouver, BC)}

Background: Intracranial berry aneurysms present rarely during childhood. They are often asymptomatic or present as a result of hemorrhage or mass effect. Cerebral infarction as a complication of thrombosis has not been reported previously.

Methods: We report a unique case of a child with acute hemiparesis secondary to cerebral infarction caused by embolism from a thrombosed intracranial berry aneurysm.

Results: A 15-month-old female infant presented with acute left hemiparesis. Her past history and development had been normal. Computed tomography demonstrated focal infarction of the right putamen and internal capsule. Angiography demonstrated a single $1 \mathrm{~cm}$ partially thrombosed berry aneurysm located immediately proximal to the bifurcation of the right middle cerebral artery at the junction of the M1 and anterior temporal artery origin. At surgery, the aneurysm was partially thrombosed with three vessels originating from the wall or dome of the aneurysm: a patent lenticulostriate artery and anterior frontal artery and a thrombosed lenticulostriate artery. The child had no other risk factors for vascular disease or thrombosis.

Conclusions: This case demonstrates embolism from a partially thrombosed intracranial aneurysm as a rare cause of cerebral infarction in childhood which has not been described previously.

\section{P-034}

\section{Asperger syndrome due to cortical dysplasia}

\section{Huh*, G Hendson, M Connolly, (Vancouver, BC)}

Background: The neuropathology of autism spectrum disorders is poorly described. Abnormal cortical minicolumnar organization, neuronal disorganization, neuronal heterotopias, and decreased Purkinje cell density are described. Epilepsy occurs in one third of cases. The objective of this report is to describe the neuropathological findings in a patient with Asperger syndrome and epilepsy.

Methods: The case records and neuropathology are reviewed.

Results: The patient developed medically refractory seizures of right frontal origin at six years. He had socialization and communication difficulties consistent with Asperger syndrome but above average cognitive skills. High-resolution brain MRI was normal. At 12 years, following invasive EEG monitoring, he had resection of the right mesial frontal lobe, anterior to the motor cortex, and is now seizure free. There has been no improvement in psychosocial development and he has developed anxiety and obsessive-compulsive disorder. Pathological analysis revealed focal cortical dysplasia with abnormal neuronal migration, neuronal clusters in the cortical layer, glial satellitosis in the cortex and white matter junction, subpial gliosis, and hypertrophied astrocytes in the white matter.

Conclusion: Focal cortical dysplasia may be associated with both Asperger syndrome and epilepsy.

\section{P-035}

\section{Recurrent ictal hemiparesis in childhood}

\section{B Landesman*, E Roland, (Vancouver, BC)}

Background: Focal paralysis is a rare negative ictal phenomenon of atonic partial seizures that may mimic ischemic events or postictal Todd's paralysis. Conventional and functional neuroimaging and electroencephalography (EEG) have demonstrated that focal atonic seizures are not well localized, and may arise from dominant and nondominant frontal, central or parietal regions.

Methods: We report an unusual case of a child who had focal atonic seizures which manifested as recurrent episodes of transient hemiparesis.

Results: This four-year-old boy presented with recurrent episodes of right flaccid hemiparesis which lasted several hours. He had mild developmental delay and a past history of neonatal seizures. Neuroimaging showed bilateral cerebral calcifications and abnormal white matter. Electroencephalography demonstrated continuous left 
posterior temporal-occipital epileptiform discharges during the time of hemiparesis. The hemiparesis and epileptiform abnormalities on EEG resolved immediately following treatment with intravenous diazepam and phenytoin.

Conclusions: Focal atonic seizures are an unusual but treatable cause of recurrent hemiparesis in children. Electroencephalography is an essential investigation to determine a possible ictal etiology.

\section{P-036}

\section{Stroke from a herbal stimulant}

N Lowry*, (Saskatoon, SK)

Background: Ephedrine containing compounds are found in various health foods. Scattered case reports suggest a relationship between ingestion of these agents and the occurrence of ischemic or hemorrhagic stroke.

Methods and Results: A previously healthy 16-year-old body builder who worked out at the gym for three days each week experienced a stroke with two ischemic infarcts, one in the right thalamus and the other in the right cerebellum. There was no evidence of dissection on MRA and his screen for hypercoaguable factors was negative. He had a four-week history of taking an herbal compound "Hydroxycut" which contains sida cordifolia, an ephedrine like agent. He took a large capsule $334 \mathrm{mgs}$, three times a day. His urine tested positive for amphetamine, which is often found with ingestion of ephedrine like substances.

Conclusions: Potent vasoconstrictors masquerade in herbal agents under unusual names such as ma huang and sida cordifolia; they carry a definite risk of stroke in young otherwise healthy individuals.

\section{P-037}

Tension-type headache (TTH) and migraine: a frequent association in children/adolescents

Shashi Seshia*, (Winnipeg, MB)

Objective: Determine relative frequency of headache (HA) types in children/adolescents referred to a Pediatric Neurologist.

Methods: (i) Study design: Prospective, observational, sequential; (ii) Setting: Private practice pediatric neurology clinic in a Canadian city; (iii) Data: Only those referred with HA between September 1998-December 2001 were entered on data sheets; (iv) I.H.S. Classification (revised) was used to type HAs; (v) Follow-up: three months to four years; (vi) those with chronic daily headache were excluded.

Results: There were a total of 320, 148 (46\%) female and 172 (54\%) male. Their age range: 2-19 years (median:11 years). Duration of HA: 1 month-14 years (median: 2 years). Migraine occurred in $118(37 \%)$, TTH alone in $52(16 \%)$. Migraine and TTH in $97(30 \%)$. Thus, 97 (44\%) of 221 with migraine as one HA type also had TTH. In 4/320, HAwas due to SOL/vascular malformation. CTs were done in $57(18 \%)$ and were abnormal in four. MRI/As were done in $12(4 \%)$ and was abnormal in one. One child (SOL) died; 25 (8\%) were lost to follow-up; 292 (91\%) were greatly improved, the majority without specific treatment.

Conclusions: Migraine and TTH frequently co-exist in children/adolescents, a factor that will influence response to specific migraine treatment in clinical trials or practice.

\section{DEMENTIA}

\section{P-038}

\section{Creutzfeldt-Jakob disease: a retrospective review}

T Townsend*, S Brass, F Andermann, (Montreal, QC)

Background: Creutzfeldt- Jakob disease (CJD) may present with the classical clinical triad of rapidly progressive dementia, myoclonus, and $1-2 \mathrm{~Hz}$ triphasic sharp waves on electroencephalogram (EEG).

Objective: To review the epidemiology, clinical features, laboratory results, and outcome of patients diagnosed with CJD.

Design/Methods: Retrospective chart review of 11 patients diagnosed with CJD. Data collection included clinical presentation, differential diagnoses, CSF analysis, neuroimaging, EEG studies, pathology, and outcome.

Results: Mean age was 61 years (range: 50-78). The female:male ratio was 8:3. Five patients underwent autopsy and had pathological confirmation of CJD. One patient was diagnosed to have the genetic variant; all others had sporadic CJD. Myoclonus, dementia and triphasic waves were present on admission in 10/11, 10/11 and 7/11 patients respectively. The classic clinical triad was initially present in $6 / 11$ patients, and in $11 / 11$ patients by discharge [mean time $=$ eight weeks (range: 0.5-32 weeks)]. Mean length of survival after symptom onset was 21 weeks (range: 5-80), and six weeks after clinical diagnosis (range: 3-9 weeks).

Conclusions: Patients with CJD are diagnosed late in their clinical course. Only 54\% of patients presented with the classic clinical triad on admission. Patients with possible CJD must be closely observed for the appearance of classical features.

\section{P-039}

Frontal lobe compensatory strategy during memory task in Alzheimer's disease

T Ortiz*, F Maestu, A Fernandez, P Gil, C Amo, (Madrid, Spain); F Quesney, (Madrid, Spain, Montreal, QC)

Background: Patients with Alzheimer's disease (AD) suffer from memory deficit affecting encoding and rapid rate of forgetfulness.

Methods: Magnetoencephalographic recording was obtained from eight $\mathrm{AD}$ patients, mean age 76 years, fulfilling NINCDS / ADRDA diagnostic criteria and from eight elderly controls, mean age 76 years. The brain magnetic activity was recorded during a high load short term memory task. Hippocampal MRI volumetric studies were performed.

Results: Control subjects showed a higher number of magnetic dipoles over the temporoparietal areas, including hippocampus, as compared to AD patients. AD patients showed a higher number of dipoles over the frontal motor areas, Broca and insula, in addition to substantial dipole reduction in the left temporal and parietal areas including the hippocampus structure which showed significant volume reduction. This pattern correlated with scores compatible with $\mathrm{AD}$ in cognitive testing (MMSE, CAMCOG) and everyday functional staging scale (FAST).

Conclusion: Our results indicate that $\mathrm{AD}$ patients compensate memory deficit by recruiting cortical networks, mostly motor, which are still spared in the early stages of the disease. The significant 
reduction of magnetic dipoles in the left temporoparietal areas in association with MRI hippocampal atrophy, represents a distinctive pattern in early stages of $\mathrm{AD}$.

\section{P-040}

Butyrylcholinesterase and homocysteine thiolactone: a risk for Alzheimer's disease

\section{$S$ Darvesh*, $R$ Walsh, E Martin, (Halifax, NS)}

Background: Elevated homocysteine is considered a risk factor for the development of Alzheimer's disease (AD). It has been suggested that a derivative of homocysteine, homocysteine thiolactone, may be more directly related to this risk. It is known that inhibition of cholinesterases improves cognition in AD and may decrease fl-amyloid deposition. We hypothesized that the detrimental effects of homocysteine metabolites may be related to alteration of the catalytic activity of cholinesterases and examined the effects of several homocysteine analogues on these enzymes.

Methods: Cholinesterases were assayed by a modification of Ellman's spectrophotometric method.

Results: Homocysteine thiolactone and its analogues, homoserine lactone and cycloserine, reversibly enhanced the activity of butyrylcholinesterase (BuChE). In contrast, homocysteine thiolactone caused irreversible inactivation of acetylcholinesterase (AChE).

Conclusions: Inhibition of BuChE has been shown to improve cognition and reduce formation of neurotoxic amyloid. The fact that homocysteine thiolactone enhances the activity of BuChE suggests that the detrimental effect of homocysteine may be due to stimulation of BuChE by this related metabolite. This strengthens the need to develop specific BuChE inhibitors for the treatment of AD.

\section{P-041}

Longitudinal change in medial temporal lobe width distinguishes mild Alzheimer's disease from normal aging: the Sunnybrook Dementia Study

Neely Bakshi*, (London, ON); Fuqiang Gao, Farrell S Leibovitch, Sandra E Black, (Toronto, ON)

Background: Longitudinal measurement of medial temporal lobe (MTL) atrophy may help in monitoring Alzheimer's Disease (AD) progression. Using a simple, standardized MRI measurement of MTL width, we prospectively examined atrophy rates in AD and normal controls (NC), in correlation with clinical progression.

Methods: Ten NC (mean age=69; MMSE=29) and 26 mild AD (mean age $=68$; MMSE $=25$ ) were serially scanned over three years. The 3D-T1 weighted MRI scans were reformatted along the long axis of the hippocampus at the inter-collicular sulcus. Anterior, posterior, midway and thinnest MTL widths between anterior and posterior borders of the midbrain were obtained, blind to serial order and clinical information.

Results: MTL width showed greater annualized decrease in AD (4.0-7.8\%/yr), compared to NC (0.9-2.4\%/yr). Decrease in posterior and midway MTLwidth was faster, accelerated in the third year (up to $23 \%$ ), and correlated more with MMSE decline ( $r=0.34-0.38$, $\mathrm{p}<0.05)$.

Conclusions: Simple serial linear MRI measurements of MTL width revealed faster rates of atrophy in mild $\mathrm{AD}$ compared to $\mathrm{NC}$, more so posteriorly, and with a nonlinear pattern showing acceleration later in the course. In conjunction with other clinical and radiological measures, MTLwidth may be useful in monitoring clinical progression in $\mathrm{AD}$, and assessing new medical therapies.

\section{EPILEPSY}

\section{P-042}

\section{Atypical neuroleptics: EEG abnormalities}

G Vorobeychik*, TRennie, R Keyes, A Prout, S Clarke, J Hooge, (Vancouver, BC)

Background: Clozapine is associated with EEG abnormalities and increased risk of seizures. Little information is published about olanzapine and risperidone.

Methods: Clinical information and EEG reports were reviewed from an EEG digital database (St. Paul's Hospital, Vancouver, Canada), which includes patients from 1997 to 2002. Patients with psychiatric diseases receiving olanzapine and/or risperidone were compared with patients on loxapine. Patients with epilepsy, organic brain syndrome, ECT, positive HIV test, delirium, or on anticonvulsant medications, were excluded.

Results: Moderate to severe EEG abnormalities in patients older than 60 years were recorded in 17 of 43 patients (39.5\%), treated with risperidone; four of 21 patients $(19 \%)$ on olanzapine, and four of 18 patients $(22 \%)$ on loxapine ( $\mathrm{p}<0.001)$. Epileptiform discharges were recorded in three out 43 patients on risperidone $(7 \%, \mathrm{p}<0.01)$. In patients younger than 60 years, moderate to severe EEG abnormalities were noted in two of 48 patients on risperidone (4.5\%) and one of 24 patients $(4.5 \%)$ on loxapine. No patients on olanzapine had moderate to severe EEG abnormalities.

Conclusion: Moderate to severe EEG abnormalities in patients above age of 60 are more frequently associated with use of risperidone than olanzapine or loxapine. No such difference was observed for patients younger than 60 .

\section{P-043}

Curvilinear multiplanar reformatting in the presurgical evaluation of refractory epilepsy patients

A Bastos, DB Sinclair, S Naik, DW Gross*, (Edmonton, AB)

Background: For patients with epilepsy who do not respond to medication, focal resective surgery provides the potential of a cure of their seizure disorder. The finding of a lesion on magnetic resonance imaging (MRI) greatly improves seizure outcome with surgery. Curvilinear multiplanar reformatting (CMPR) is a new method of image processing that allows simplified visualization of the brain's convolutions, thereby facilitating the detection of cortical lesions. We present two cases in which CMPR allowed the detection of subtle lesions that had not been detected with conventional imaging techniques.

Methods: Volumetric T1 weighted MRIs were first analyzed using conventional rectilinear slices and subsequently using CMPR.

Results: Two patients with frontal lobe epilepsy and normal volumetric MRI studies using conventional rectilinear slices were studied. In both cases, analysis of the volumetric MRI using CMPR demonstrated subtle focal frontal lesions. In case one, the lesion was 
consistent with an old ischemic event, while in case two, the lesion was consistent with Taylor type focal cortical dysplasia.

Discussion: CMPR is an important clinical tool that provides information not apparent with conventional rectilinear imaging techniques. In detecting lesions that had not been apparent, it is expected that CMPR will result in improved surgical outcome for medically intractable patients.

\section{P-044}

\section{Prolonged deficits after focal inhibitory seizures}

Miguel Bussiere*, David Pelz, Robert Reid, Bryan Young, (London, $O N)$

Background: Seizures are most commonly associated with positive phenomena such as tonic, clonic or myoclonic movements, automatisms, paresthesias and hallucinations. Negative phenomena, however, are not an uncommon manifestation of seizure activity. Examples of negative seizure phenomena include speech arrest, aphasia, amaurosis, amnesia, numbness, deafness, neglect and atonic seizures. Less commonly described in the literature are focal inhibitory motor seizures.

Methods: Two patients presenting with rapidly progressive, prolonged hemiparesis, sensory neglect and hemi-visual field obscuration are described.

Results: Computed tomography (CT) and magnetic resonance imaging (MRI) of the brain did not reveal progression of known structural lesions or new lesions. The superficial cortex of the hemisphere contralateral to the hemiparesis and sensory neglect enhanced diffusely with gadolinium on T1-weighted MRI images. Electroencephalography (EEG) demonstrated background slowwave activity and single photon emission computed tomography (SPECT) revealed hyperperfusion in the hemisphere contralateral to the hemiparesis and sensory neglect. The changes seen on MRI and SPECT resolved with resolution of the symptoms.

Conclusions: Taken together with the clinical history, the results from these investigations suggest prolonged focal inhibitory seizure as the underlying etiology. A review of the literature and investigations helpful in making this difficult diagnosis will be provided.

\section{P-045}

\section{A case of a challenging EEG in psychiatric patient}

\section{G Vorobeychik*, A Prout, R Keyes, T Rennie, (Vancouver, BC)}

Background: EEG abnormalities in psychiatric patients might be related to medication use or underlying diseases.

Methods: Case presentation

Results: A 41-year-old right-handed male with long history of mood swings consistent with bipolar disorder presented with decreased verbal output (mutism) and psychotic features. On admission, he was started on rispiridone, loxapine and lorazepam. On his initial EEG bilateral frontal irritability (sharp, sharp and slow, and slow waves) was present throughout the entire record. CT, MRI, MR angio, blood and CSF work up for inflammatory conditions were all negative. He remained on the same medications. Tegretol and Epival were added to his regimen but the patient was not compliant. Three weeks later his EEG showed only nonspecific changes bilaterally (fast activity and alphoid discharges in the frontal regions). Later, the patient was treated with ECT and significantly improved. He was discharged home without medications (patient refused to take medications). His follow-up EEG is pending.

Conclusions: Moderate or severe EEG abnormalities in psychiatric patients can be difficult to interpret. They can be related not only to neurological co-morbidities (as was suspected initially in our case) but also to primary psychiatric disorder and/or use of antipsychotic medications.

\section{P-046}

\section{Lamotrigine audit study}

B Young*, (Toronto, ON); Z Hutchinson, S Levin, W Blume, (London, $\mathrm{ON}$ )

Background: Lamotrigine (LTG) is thought to be effective against a wide spectrum of seizure types.

Methods: Retrospective chart review of pediatric and adult outpatients on LTG for $>6$ months.

Results: Of 15 children, 12 had Lennox-Gastaut syndrome; others had absence, complex partial or secondary generalized seizures. LTG monotherapy was successful in only one child (7\%); one became seizure-free and four (27\%) had greater than $50 \%$ reduction in seizures. LTG was withdrawn in four $(27 \%)$ children because of lack of effectiveness and in one for behavioral side effects. Of 55 adults nine had Lennox-Gastaut syndrome, 22 had complex partial seizures, 19 had generalized convulsions, three had myoclonic epilepsy and one had absence (not mutually exclusive). Sixteen (29\%) adults became seizure-free when on LTG, eight as monotherapy (all had $<4$ seizures/month). Twenty (36\%) had greater than $50 \%$ reduction in seizure frequency. LTG was withdrawn in 16 cases for: ineffectiveness in $12(22 \%)$, associated pregnancy in two, lymphadenopathy in one and behavioral changes in one.

Conclusions: LTG is effective for both focal and primary generalized seizure disorders in both adults and children, especially with low seizure frequency. In an equal number of patients the drug is ineffective.

\section{P-047}

\section{Topiramate audit study}

\section{B Young*, (Toronto, ON); Z Hutchinson, W Blume, (London, ON)}

Background: Topiramate (TPM), a relatively new anti-epileptic drug, has a variety of actions and should be effective for many seizure types.

Methods: Retrospective chart review of adult outpatients on TPM for epilepsy.

Results: There were 46 adults (22 men and 24 women) aged 1669 years with various seizure types. Monotherapy was attempted in five and was successful (no seizures) in three patients. Three adults became seizure free, 15 had a $>75 \%$ reduction, 10 between $50-75 \%$ reduction and no change in seizure frequency in the remainder. Some patients in each seizure type showed $>50 \%$ reduction in seizure frequency. The drug was withdrawn in $12(26 \%)$ cases, in seven because of ineffectiveness and in five because of adverse effects. Side effects, not necessarily requiring discontinuation and not mutually exclusive, included fatigue/sedation in nine, weight loss in four, cognitive changes in three, tremor in two and behavioral changes in two. 
Conclusions: Topiramate is effective against a wide variety of seizure types, and has a rate of withdrawal /discontinuation that is similar to other drugs.

\section{P-048}

\section{Subtraction ictal SPECT coregistered onto MRI (SISCOM) provides clinically important information in epilepsy patients being investigated as candidates for surgery}

DW Gross*, M Hoskinson, C MacGuire, SN Ahmed, D Quigley, (Edmonton, $A B$ )

Background: SISCOM is an important tool in the investigation of candidates for epilepsy surgery. However, as successful studies require injection less than one minute after seizure onset, many centres have difficulties obtaining consistent results. We report the one year experience of SISCOM performed at the University of Alberta between January 1 to December 31, 2002.

Methods: Ictal injections were planned for days when medication was rapidly reduced with a nurse trained to perform the ictal injections remaining at the bedside for the entire day. SISCOM images were generated offline using the Philips LX workstation.

Results: SISCOM was attempted on 24 patients with ictal injections being obtained in 14 cases. The mean time from clinical seizure onset to radioisotope injection was 22 seconds (range 9-30s). Of the fourteen successful injections, eight demonstrated localized ictal cerebral hyperperfusion, which correlated with the rest of the localizing information.

Conclusions: SISCOM is an important tool in the presurgical evaluation of epilepsy patients. Planning of the study with a nurse at the bedside provided excellent timing of injections with the drawback being that manpower limitations limited the number of days when the test could be attempted (reducing the chances of obtaining an ictal injection).

\section{P-049}

\section{Hazard of marihuana in patients with epilepsy}

\section{A Ogunyemi*, (St John's, NL)}

Background: The use of marihuana to treat epileptic seizures, and other medical conditions, has been thrust into the spotlight by the public media. There are no persuasive clinical trial results to support the use of the drug to treat epilepsy. The indications, contraindications, precautions and toxic reactions for patients with epilepsy remain to be clarified.

Methods: We evaluated at the Seizure Clinic, Health Sciences Centre, St. John's, three patients with epilepsy who experienced severe adverse outcomes as a result of use of marihuana coupled with noncompliance with their anti-seizure medications. Patient 1 , a 26-year-old man had cryptogenic temporal lobe epilepsy. Patient 2, a 25 -year-old man had idiopathic generalized epilepsy. Patient 3, a 46-year-old man had partial seizures caused by multiple intracranial meningiomas related to type 2 neurofibromatosis.

Results: Patient 1 was found dead in his home with the paraphernalia of marihuana smoking beside his body. Patient 2 experienced serial generalized tonic clonic seizures. Patient 3 suffered complex partial status epilepticus. Both Patients 2 and 3 admitted extensive use of marihuana and lack of adherence to their anti-seizure medications. They recovered without sequelae.
Conclusions: Epilepsy patients should be aware that the use of marihuana coupled with failure to adhere to standard antiepileptic medications may result in disastrous consequences. There is a need to conduct well-designed clinical trials to evaluate the safety and efficacy of marihuana for epilepsy patients.

\section{P-050}

\section{Cessation of smoking a byproduct of EEG telemetry} monitoring

SN Ahmed*, D Quigley, ZA Siddiqi, (Edmonton, AB)

Background: Smoking is a common problem in epilepsy patients. Inpatient video EEG monitoring (VEEG) unit provides a unique and conducive environment to epilepsy patients to actively participate in a smoking cessation program. Two patients are reported who were able to quit smoking successfully during admission for VEEG.

Methods: A 26-year-old woman with an eight-year history of smoking and a 48-year-old man with a 25-year history of smoking were admitted for VEEG for seven days. The first patient had not tried any organized programs previously to quit smoking, while the second patient had ineffectively tried hypnosis and nicotine gum in the past. As part of smoking cessation efforts both were counseled for potential risks of smoking and given $18 \mathrm{mg}$ nicotine patch daily. The patients were monitored round the clock and remained confined to their rooms for VEEG. In spite of excessive craving to smoke both patients did not smoke during their hospital stay. Both were counselled again at discharge and provided with a seven-day supply of nicotine patches.

Results: These patients did not resume smoking at follow-ups ranging from 5-6 months.

Conclusions: The unique milieu of VEEG unit provides an opportunity to the physician to counsel their epilepsy patients to quit smoking.

P-051

Opioid therapy in a patient with chronic intractable seizure and headache disorders results in excellent control of both disorders

\section{Wine*, (Saskatoon, SK)}

Background: In a general adult neurology practice setting, patients are not uncommonly encountered with refractory seizure and/or pseudoseizure disorders using standard or add-on anticonvulsants alone or in combination. Not uncommonly, such patients may have comorbid severe chronic headache disorder refractory to standard therapies for headache.

Methods: A case presentation of a 48-year-old female with welldocumented and refractory symptomatic seizure disorder as well as a severe and refractory chronic headache/regional hyperalgesia disorder

Results: The addition of an oral high dose opioid analgesic dramatically improved both conditions from the beginning and after 21 months follow-up to date.

Conclusions: Opioid analgesics are a safe and useful adjunct to consider in treating this and other patients with otherwise refractory seizure and hyperalgesia disorders. Valid reasons why opioid analgesics may be considered as rational therapy for seizure disorder in the presence of regional or generalized hyperalgesia disorders will 
be outlined. Possible and invalid reasons why, until now in this patient, opioids had been withheld for the chronic headache disorder and therefore not considered as therapeutic for both disorders, will be explored.

\section{P-052}

\section{Does MEG reliably localize comprehensive language?} A validation study with the Wada test

F Maestu, T Ortiz*, A Fernandez, C Amo, (Madrid, Spain); F Quesney, (Madrid, Spain, Montreal, QC)

Introduction: Several neuroimaging techniques such as PET, fMRI and MEG are currently used for brain language localization. Of these, only MEG provides enough spatio-temporal resolution to reliably localize ongoing comprehensive language processing down to the millisecond level. Our purpose was to compare MEG speech localizing data with Wada test results.

Material and Methods: 21 patients with intractable epilepsy underwent MEG language mapping. A subset of eight patients also underwent Wada test.

Results: $2 / 21$ patients showed right hemisphere dominance. The remaining (19), showed left hemisphere dominance for language on MEG recording. Wada test was in perfect agreement with MEG results in all patients but one (7/8 patients). This patient had a right temporal cortical dysplasia with right hemispheric language lateralization on MEG. Wada test, however, failed to show hemispheric dominance. Following a right temporal basal removal, this patient suffered a transient global aphasia.

Conclusions: As compared to Wada test, MEG reliably lateralizes language function in epileptic patients undergoing presurgical evaluation. This technique, in addition to being noninvasive, is unique since it can identify the different anatomical structures involved in language processing as a function of real time. This provides crucial cues regarding the functional organization of the cortical network processing language.

\section{P-053}

\section{Clinical significance of isolated hippocampal volume asymmetry in childhood epilepsy}

P Major*, J-C Decarie, P Diadori, A Lortie, P Cossette,

\section{$L$ Carmant, (Montreal, QC)}

Background: Hippocampal asymmetry (HA) without sclerosis is considered a precursor of mesial temporal sclerosis (MTS). The goal of this study is to define the clinical characteristics and evolution of epileptic children with isolated HA.

Methods: MRIs were reviewed blindly without quantitative measurements by one neuroradiologist. Seizure history, EEGs, risk factors and outcome were recorded.

Results: Seventeen charts were reviewed. Four were excluded after MRI review (two MTS, one ulegyria and one unavailable films). Six boys and seven girls were included. Eleven had temporal lobe epilepsy. Interictal discharges were ipsilateral to the lesion in three and contralateral in two. Bilateral discharges occurred in five and one had normal EEGs. Two had generalized epilepsy. Mean age at beginning of seizures was 7.2 (range: 2.5-15). One had a past history of febrile seizures. Nine had a positive family history of seizures. Seizure control with one anticonvulsant was achieved in 12 (mean follow-up = five years).

Conclusion: In our cohort, children with HA in the absence of sclerosis rarely go on to develop refractory epilepsy. The association of a positive family history, low incidence of febrile seizures and benign prognosis point to a distinct epileptic syndrome. We intend to perform genetic studies in these families.

\section{P-054}

\section{D-reconstruction of magnetic source image data for intra-} operative navigating system

Koji Iida*, Hiroshi Otsubo, Stepanie Holowka, Yuuri Matsumoto, James T Rutka, O Carter Snead III, (Toronto, ON)

Purpose: Magnetic source imaging (MSI) is a combination of magnetic sources of evoked responses and epileptic discharges coregistered with structural magnetic resonance images (MRI). We evaluated the usefulness of 3D-reconstruction of MSI data for intraoperative navigating system in paediatric patients with intractable epilepsy.

Methods: We studied magnetoencephalography (MEG) (Onega 151, CTF Systems Inc., Port Coquitalam, BC) to obtain somatosensory evoked fields (SEF) and interictal spikes in 16 patients with (14) or without lesions (2). MEG data were overlaid onto individual T1 weighted MRI. We converted MSI data on a neurosurgical flameless stereotaxy system (ISG Allegro 3-D imaging software, Sedara Systems Inc., Mississauga, ON) in the operating room.

Results: All 16 patients underwent cortical excision using intracranial EEG (5) and lesionectomy (11). The epileptogenic zone was located around the sensorimotor cortex in 11 patients. In 10 of them, magnetic SEF identified the central sulcus confirmed intraoperatively. MEG showed epileptic spike sources around the sensorimotor cortex in seven of the 11 patients. MEG and intraoperative electrocorticography showed colocalized interictal spikes to demarcate the epileptogenic zone in seven patients.

Conclusions: 3D-reconstruction MSI data for intra-operative navigation system can precisely localize functional and epileptic regions on the exposed brain surface. This technique allows the neurosurgeons to plan the optimal preoperative trajectory and to perform intra-operative demarcation of the epileptogenic lesions, especially around the eloquent area.

\section{P-055}

\section{Precognition as an epileptic aura}

M Sadler*, S Rahey, (Halifax, NS)

Background: A myriad of subjective experiences comprise the auras of temporal lobe originating seizures. A patient with an aura of precognition (prescience) was encountered. This experience prompted a review of this ictal phenomenon in patients attending a tertiary care epilepsy outpatient clinic.

Methods: A computer epilepsy database was searched for patients with simple partial seizures and complex partial seizures with auras. Identified patients had their charts reviewed for details of their auras. Literature searches (PubMed) were done using the search terms "precognition" (or "prescience") combined with "seizures" or "epilepsy." Standard comprehensive epilepsy textbooks were also reviewed. 
Results: The charts of 218 patients (from the database of 927 patients) were reviewed. Three patients had precognition as an ictal feature. The patients' descriptions were very similar in all cases (a profound sense of being able to forecast the immediate future of their environment). The experience was clearly distinct from deja vu and other psychic experiences. All patients likely have temporal lobe epilepsy. No other description of precognition as an ictal feature was found in the literature.

Conclusions: Precognition occurs in a minority of reports of patients with temporal lobe epilepsy and represents a previously undescribed psychic phenomenon.

\section{P-056}

\section{Utilization of CT of the head at the time of first seizure by emergency room physicians at the Children's Hospital of Eastern Ontario}

\section{E Belanger*, R Brannan, C Pitters, D Keene, (Ottawa, ON)}

Background: This study was to investigate the utilization of CT of the head in children who presented to a busy pediatric emergency room with first seizure.

Methods: Names of patients who were seen in the Emergency Room of the Children's Hospital of Eastern Ontario between April 1999 and March 2001 for "seizures" was obtained. A retrospective review of their medical record was done. Age at time of visit, type of seizure, cause of seizure, whether CT of head was done and results of CT were obtained. To be included in this review, the child had to be over 28 days and under 18 years of age, a first time seizure and not have a previously diagnosed neurological disorder.

Findings: Over the study period there were over 100,000 emergency visits logged of which 724 patients records were identified for review. 75 patients with first time seizure had a CT scan done. 100 presented with unprovoked first seizure and did not have CT done. 312 patients had febrile convulsions without having had a CT done. The remainder of the patients were excluded due to not meeting age requirements of review or had an underlying neurological disorder.

\section{P-057}

\section{Mild head trauma and seizures}

\section{P Tai ${ }^{*}$, D Gross, (Edmonton, $\left.A B\right)$}

Background: While the risk of developing epilepsy following a mild head injury is low, the effects of mild head injury on patients with pre-existing epilepsy have not been reported. We present a case series where a striking temporal relationship between mild head injury and worsening of seizure frequency was observed.

Methods: Five cases were identified and hospital and clinic charts reviewed in detail.

Results: One patient has primary generalized epilepsy and four have localization related epilepsy. Prior to the head injury, three patients were seizure free (range: two to 24 years). In all cases, the head injury was mild with no or transient loss of consciousness and no focal neurological deficits. In all cases, the patients experienced a worsening of seizure control within days of the injury, with four of five cases remaining medically refractory since.

Conclusions: A close temporal relationship between mild head injury and a worsening of seizure control was observed in five epileptic patients. This observation suggests that a head injury that would be considered benign in the general population can have serious consequences in patients with epilepsy such as recurrence of seizures and medical intractability.

\section{P-058}

Outcome after corpus callosotomy in the pediatric population

A Lary*, BM Wheatley, K Aronyk, B Sinclair, T Snyder,

JDS McKean, (Edmonton, $A B$ )

Background: Corpus callosotomy is variably effective for controlling seizures in pediatric patients with medically intractable epilepsy.

Methods: Between 1991 and 2002, eight patients were investigated and then had corpus callosotomies at the Comprehensive Epilepsy Program at the University of Alberta. All patients had long term video EEG, MRI, and neuropsychological assessments prior to surgery. Three patients had Lennox-Gastaut syndrome. Two patients had porencephalic cysts. Most had multiple seizure types.

Results: The mean follow-up has been 6.8 years. Four patients had only transient improvement (six months). Two patients were improved for five years but have since had a recurrence of seizures. Two patients have had a marked improved. The outcome for patients with drop attacks was more favourable than for patients with other seizure types. Five patients had complete callosotomies. All patients had moderate to severe mental retardation pre-operatively with no improvement postoperatively. One patient had left sided hemiplegia secondary to venous infarction.

Conclusions: In the pediatric population, the outcome from corpus callosotomy is marginal.

\section{EPILEPSy / General NeUrology}

\section{P-059}

\section{Insulinoma misdiagnosed as epilepsy}

M-H Dion*, P Cossette, J-M St-Hilaire, DK Nguyen, (Montreal, $Q C)$

Background: Many conditions may mimic epileptic spells. Among them are hypoglycemic spells which may lead to a variety of neuroglycopenic symptoms such as confusion, personality change, and bizarre behavior.

Methods: We report here three patients initially misdiagnosed as epileptic who were subsequently found to have hypoglycemiarelated spells due to an underlying insulinoma. The medical record of each patient was reviewed with interest on clinical presentation and investigations leading to diagnosis.

Results: All three patients consulted for paroxysmal episodes of abnormal behavior. Spells varied in semiology from personality changes, to emotional lability, confusion, to awkward behaviors (videos will be shown). Basic interictal neurological investigations failed to detect any major abnormalities and patients were thought to have epilepsy. All were started on anticonvulsants for months. Continuous audio-video EEG monitoring and ictal glucose concentration measurements eventually allowed the correct diagnosis of hypoglycemia-related spells and all three were found to 
have an insulinoma upon further investigations. Delay in diagnosis ranged from six months to nine years. All patients were cured following surgical removal.

Conclusions: The diagnosis of insulinoma should be considered in patients with unusual or inexplicable neurological symptoms. The causes and consequences related to the delay in diagnosis are discussed

\section{General Neurology}

\section{P-060}

Internuclear ophthalmoplegia, abnormal CNS MRI and multifocal motor neuropathy in association with infliximab and methotrexate treatment

C Voll*, R Triaforos, (Saskatoon, SK)

Objective: We report a patient who developed clinical and MRI features of CNS demyelination in conjunction with a multifocal motor neuropathy after treatment with infliximab and methotrexate for rheumatoid arthritis.

Methods: Case report and review of literature.

Results: A 50-year-old female developed an internuclear ophthalmoplegia and a motor neuropathy with conduction blocks six months after beginning treatment with infliximab. MRI demonstrated features consistent with a central demyelinating process and the CSF showed a mild lymphocytic leucocytosis. The motor neuropathy responded to treatment with intravenous gammaglobulin (IVIg), however she continues to require monthly infusions 18 months after discontinuing treatment with infliximab.

Conclusions: The temporal association between the onset of treatment with infliximab supports an etiological role for infliximab in the neurological presentation in this patient. This reports emphasizes the need for awareness that infliximab may cause central and peripheral neurological disease.

\section{P-061}

\section{Evaluation of enzymes in meningitis}

Manju Sharma*, Nitya nand Sharma, (New Delhi, India)

Lactic dehydrogenase (LDH), creatinine phosphokinase (CK) and gama glutamyl transpeptidase (GGTP) were measured serially $(0,4,7$ th day) in cerebrospinal fluid (CSF) and serum in 23 cases of meningitis (15 pyogenic and eight tuberculous meningitis) and an equal number of age and sex matched healthy control subjects to find out their diagnostic and prognostic significance in cases of meningitis.

Lactic dehydrogenase, creatinine kinase and gamma glutamyl transpeptidase were estimated colorimetrically using Kits, both in serum and cerebrospinal fluid.

The levels of these enzymes were significantly elevated in cases of meningitis in serum and CSF as compared to control subjects. The activity was significantly higher in pyogenic than tuberculous meningitis $(p<0.001)$ and in CSF than in serum $(p<0.001)$. The maximum elevation in activity of GGTPand LDH were seen on the first day, whereas CK was highest on fourth day and thereafter the activity of all enzymes declined in majority of cases. However, in three cases of pyogenic and five cases of tuberculous meningitis, the enzyme activity on subsequent estimation increased serially. All these eight cases died. Further, the basal enzymatic activity in all eight cases who died, was higher as compared to those who survived. Of these enzymes, CSF GGTP levels correlated best with the clinical picture.

It is concluded that GGTP, CK and LDH are significantly elevated in cases of meningitis. It was not possible to differentiate the type of meningitis on the basis of activity in any of them. However, it was possible to predict prognosis. Significantly higher basal activity and serial rise were associated with poor prognosis.

P-062

\section{Cerebrospinal fluid cytokines in carcinomatous meningitis}

KM Yoo*, MJ Kim, BG Yoo, K Kim, (Busan, Korea)

Background: Carcinomatous meningitis (CM) is the least common form of intracranial metastatic disease. The early diagnosis may produce symptomatic improvement and prevent neurological deterioration. The purposes of this study are to suggest the useful diagnostic method through analyzing cerebrospinal fluid (CSF) cytokines of $\mathrm{CM}$, and to evaluate the correlation among cytokines, biochemical markers, and tumor markers.

Methods: Sixteen samples were evaluated in 12 patients with CM. The interleukin (IL)-6, IL-8, IL-10, tumor necrosis factor (TNF)-alpha and monocyte chemoattractant protein (MCP)-1 were measured by sandwich ELISA method. The diagnostic values of each cytokines were compared with other markers.

Results: The values of CSF IL-8 and MCP-1 were significantly higher in CM compared with control group ( $\mathrm{P}<0.005)$. CSF IL-6 in $\mathrm{CM}$ was higher than in control group. IL-10 was negative in all cases. Carcinoembryonic antigen (CEA) was elevated in 13 samples. There was no significant relationship between cytokine levels and other diagnostic values.

Conclusions: CSF IL-6, IL-8 and MCP-1 are a valuable tool of the investigation of $\mathrm{CM}$ in cancer patients and CEA is a useful diagnostic parameter in $\mathrm{CM}$. Therefore a rapid assay for the above cytokines and tumor marker may contribute to the early diagnosis of CM.

\section{P-063}

\section{Influence of a mental numberline on vertical line bisection}

M San Vicente*, A Kirk, (Saskatoon, SK)

Background: We attempted to determine whether number magnitude biases normal subjects'vertical line bisection accuracy.

Method: Experiment One: Subjects bisected sixteen vertical strings of numbers randomly distributed on a page. Each string of digits was made up of the numbers one, two, eight or nine, all of the digits in each string being the same digit. Experiment Two: Subjects bisected solid vertical lines with digits flanking the endpoints of each line. In both experiments, the numbers one and two represented small magnitude numbers and eight and nine large.

Results: Experiment One: There was no significant difference between bisection of digit strings composed of high versus low magnitude numbers with an upward bias in both conditions. Experiment Two: Small value numbers induced an upward bisection bias and large numbers a downward bias.

Conclusions: Experiment One suggests that high versus low magnitude vertical number strings do not affect judgment of 
midpoint. In Experiment Two, there was a distinction between lines flanked by small and large numbers, potentially due to the activation of an association of number magnitudes with spatial position. It appears that a mental spatially coded number line exists with higher numbers at the bottom and lower numbers at the top.

\section{P-064}

\section{HIV-1/AIDS neuropathology in a Canadian teaching centre}

KA Walsh*, W Thompson, J Megyesi, JX Wilson, R Hammond, (London, ON); C Wiley, (Pittsburgh, PA)

Background: The nervous system is a major target of HIV-1/ AIDS. Most data on the range and frequency of neuropathology in HIV-1/AIDS comes from large centres outside Canada in different social and health care settings. The goal of the present study was to describe HIV-1/AIDS-associated neuropathology at a Canadian teaching centre.

Methods: The records of the Department of Pathology, London Health Sciences Centre were electronically searched for cases of HIV-1/AIDS that came to postmortem examination in the preceding 10 years. The clinical records and pathological materials were reviewed.

Results: Fifteen autopsies of HIV-1/AIDS were identified. All patients were male. Fourteen contracted HIV-1 through high risk homosexual activity and one through transfusion of blood products. Twelve cases had neuropathological findings. The most common diagnoses were HIV-1 encephalitis, progressive multifocal leukoencephalopathy, toxoplasmosis, and primary CNS lymphoma with examples of multiple concurrent neuropathological diagnoses. Two patients had premortem evidence of HIV-1 associated dementia.

Conclusions: In this autopsy series, high risk homosexual behaviour was a more prominent risk factor than in previous reports from other centres where injection drug use and high risk heterosexual activity factored prominently. This study confirms the heavy burden and wide range of neuropathology in the setting of HIV-1/AIDS. The incidence of HIV-1 associated dementia was similar to that reported previously.

\section{P-065}

Progressive encephalomyelitis with rigidity (PREM) in a young woman with GAD-65 antibodies and other autoimmune dysfunction

\section{T Morrison*, B Young, (Toronto, ON)}

Background: Progressive encephalomyelitis with rigidity (PREM) is one a group of diseases that include the stiff-person (formerly stiff-man) syndrome. GAD-65 antibodies are commonly found in stiff-person syndrome, often associated with a paraneoplastic syndrome. We present a young woman who has other manifestations of autoimmune disease and PREM without evidence of malignancy.

Method: Case report and literature review.

Results: This currently 23-year-old woman developed Graves' thyroiditis treated with radioactive iodine in September 2001. In June 2002 she developed a profound memory disturbance and typical imaging features of bilateral limbic encephalitis. Investigations were negative except for GAD-65 antibodies in serum. She returned with convulsive status epilepticus in October 2002. Two months later with progressive back pain, twitching, and enhanced startle response. She progressed rapidly to severe spasms and opisthotonic posturing, raised CK and early renal failure. She required intubation and paralysis to control her spasms. IVIG and pulsed steroids were unsuccessful. A paraneoplastic work-up was again negative. High titers of GAD-65 antibodies were again found. Intrathecal baclofen was used to control her spasms. She subsequently developed brachial neuritis involving the upper trunk.

Conclusions: This unique individual developed four temporally dispersed auto-immune diseases: Graves disease, limbic encephalitis, stiff-person syndrome and brachial neuritis. There is likely a severe problem with immune surveillance mechanisms that has yet to be defined.

\section{P-066}

Spontaneous intracranial hypotension presenting as reversible coma: an unusual presentation

J Kashmere, DW Gross*, M Jacka, D Emery, (Edmonton, AB)

Background: Spontaneous intracranial hypotension $(\mathrm{SIH})$ is a well recognized neurologic disorder that typically presents with orthostatic headaches, low cerebral spinal fluid pressures, and distinct abnormalities on magnetic resonance imaging.

Methods: We present a case of an unusual presentation of SIH.

Results: A 49-year-old man presented with a two week history of orthostatic headaches that rapidly progressed to encephalopathy and coma, requiring intubation. Neuroimaging revealed abnormalities typical of SIH; diffusely enhancing meninges, subdural fluid collections, and descent of the posterior fossa. Treatment with an epidural blood patch reversed his coma within minutes. Following a second blood patch, the patient became asymptomatic. No cerebral spinal leak could be identified on MRI or on a technetium CSF flow study. At three month follow-up, he remained entirely symptom free.

Conclusions: The mechanism of coma in SIH is presumed to be compression of the diencephalon from downward displacement of the posterior fossa. Although it is very unusual for patients with SIH to present with coma, it is important to recognize since the coma may be reversible with epidural blood patches.

\section{P-067}

Resolution of see-saw nystagmus and chiasmal visual loss from hydrocephalus

James A Sharpe*, Jeong-Min Hwang, (Toronto, ON)

Background: Bitemporal visual field defects due to a dilated third ventricle were recognized in 1931 by Sinclair and Dott. See-saw nystagmus, originally described by Maddox in 1913 in a patient with bitemporal hemianopia, consists of cycles of intorsion and elevation of one eye with synchronous extorsion and depression of the other eye. Pendular see-saw nystagmus is most often associated with chiasmal lesions. We report both chiasmal visual loss and see-saw nystagmus from a dilated third ventricle.

Methods: Case study by video-oculography and MR imaging.

Results: A 22-year-old man with congenital aqueductal stenosis underwent third ventriculostomy at the age of 18. At age 20 he developed papilloedema and headaches. MRI showed hydrocephalus with a ballooned lamina terminalis of the third 
ventricle displacing the optic chiasm anteroinferiorly. He underwent another third ventriculostomy and ventriculo-peritoneal shunting. During the course of the elevated intracranial pressure (ICP), the patient's vision declined in both eyes. Visual acuity was 20/100 in the right eye and 20/200 in the left eye. Visual fields showed dense bitemporal hemianopia. He had large amplitude pendular nystagmus composed of intorsion and elevation of one eye with synchronous extorsion and depression of the other eye. Four months later, his best-corrected visual acuity had improved and the see-saw nystagmus had ceased. One year later visual acuity was 20/30 in the right eye and 20/50 in the left. Goldmann visual field examination showed only small temporal hemianopic scotomas in each eye.

Conclusions: Visual loss with bitemporal hemianopia implicated chiasmal compression by an enlarged third ventricle. See-saw nystagmus is attributed to compression of the chiasm and rostral midbrain by distention of the third ventricle. Visual loss and nystagmus resolved after intracranial pressure was relieved by shunting. The disappearance of see-saw nystagmus and resolution of bitemporal hemianopia indicates that these deficits can be reversible.

\section{General Neurosurgery}

\section{P-068}

\section{Superficial siderosis of the CNS secondary to a bleeding pseudomeningocele: a case report and review of the literature}

M Kole*, S Lownie, (London, ON)

Background: Superficial siderosis of the CNS is caused by chronic bleeding into the subarachnoid space. This disease entity is most commonly associated with tumors, particularly ependymomas, vascular anomalies, including AVMs, cavernomas of the brain and spine and post hemispherectomy. However, the source of bleeding often eludes detection. Various dural abnormalities, such as cervical nerve root avulsions and pseudomeningoceles are often overlooked, but can represent a substantial source of chronic bleeding into the subarachnoid space.

Methods: We reviewed all the cases of superficial siderosis of the CNS reported in the literature presumed to be secondary to a traumatic root avulsion or pseudomeningocele and found a total of nine cases including our case report.

Results: All of the reported cases were young males, at the time of traumatic injury (age range 20-23, average age 21.5 years old). Five patients suffered single nerve root avulsion and three patients suffered multiple nerve root avulsions. One patient developed a pseudomeningocele following lumbar laminectomy. The C8 nerve root was the most commonly involved nerve root $(n=5)$. The interval from the time of injury to the onset of symptoms ranged from eight to 28 years with an average time interval of 16.7 years. The most common symptom was ataxia followed by sensorineural hearing loss. Four patients experienced anosmia. Three patients experienced genitourinary dysfunction or impotence. Dysarthria was present in two cases. All patients demonstrated bloody or xanthochromic CSF on analysis. Of these cases only three have been treated surgically and only one case definitely identified the source of chronic bleeding. We present the first case of superficial siderosis of the central nervous system caused by a traumatic nerve root avulsion with intra-operative confirmation of the source of chronic bleeding.

Conclusions: Although, the development of superficial siderosis of the CNS, as a delayed sequela of a traumatic nerve root avulsion is rare, the clinician and surgeon should be familiar with this syndrome.

\section{P-069}

The early experience of using the EMFsystem in microsurgical resection of brain tumors

\section{Khashab*, (Khobar, Saudi Arabia)}

This presentation is about the value of electromagnetic field (EMF) in resection of brain tumors. This system is a $13.56 \mathrm{MHz}$ radiofrequency generator. The system can be used with success in resection of brain tumors and is most valuable with the removal of tumors which have a harder consistency. The pinpoint heating does not extend to surrounding structures and hence does not cause any injury to surrounding structures. This system allows neurosurgeons to coagulate and to cut through tumors but also to vaporize and to have a lesser degree of bleeding during surgery. The system had been used in seven cases of brain tumors and it had shown itself as a user-friendly and valuable adjunct tool during the resection process. With comparison to the usual conventional methods such as bipolar diathermy, the EMF was found to be an extremely useful tool in cases where the tumor showed hard consistency and where the tumors appeared to be vascular in nature. The multiple attachments to the system allowed us to be able to perform a wide variety of manipulations during microsurgery. We recommend the further use of this system which is an additional useful adjunct to the surgeon's armamentarium.

\section{P-070}

Brain abscess caused by a new species of Nocardia: case report S Khan*, S Sanche, C Robinson, F Pirouzmand, (Saskatoon, SK)

Objective: Cerebral nocardial abscesses are very rare, especially in immunocompetent individuals. Their incidence, however, is rising. We report the first known case of cerebral abscess caused by a newly identified strain. Surgical intervention and an extended course of antibiotic therapy yielded an excellent outcome.

Clinical presentation: A 63-year-old man presented with a twoweek history of subtle personality changes, uncharacteristic quietness, declined energy, gait ataxia and a possible seizure. Radiologic investigations manifested a right frontal multiloculated ring enhancing lesion with significant surrounding vasogenic edema. Preoperatively, the presumptive diagnosis was of a neoplasm.

Intervention: Right frontal craniotomy was performed. Creamy, yellowish, odorless discharge from a multi-cystic lesion was encountered. Partial resection was done and multiple specimens of the discharge and cyst wall sent for analysis.

Conclusions: Microbiology cultures yielded Nocardia paucivorans. Postoperatively, the patient was started on intravenous antibiotics including ceftriaxone and metronidazole, which were changed to trimethoprim/sulfmethoxazole on availability of pathology results. This is the first documented case of intracerebral abscess by species paucivorans, genus Nocardia. Infection of an immunocompetent individual points to nocardiosis remaining a consideration in the differential diagnosis of contrast enhancing lesions. We demonstrate that a residual nocardial abscess can be cured with prolonged antibiotic therapy. 


\section{Peer review in medical publications}

E Berger*, (Montreal, QC)

Background: When submitting a paper for publication the journal's 'instructions to authors' must be properly followed to improve the chances of acceptance.

Methods: Verify rules regarding authorship, check lists, conflict of interest, key words, abstract, title, lay out, accompanying letter. Read the paper several times to make sure there are no typing mistakes or other errors. When the study involves randomised controlled trials (RTCs), ascertain if randomization methods were properly interpreted including allocation of subject. State if blinded outcome assessment was used. Explain if results statistically or clinically significant. Other factors to be considered are 1) historical evolution of peer review, 2) composition of the peer review committee, 3) criteria for selection of a scientific paper, 4) the amount of time devoted to peer review, 5) acceptance and rejection, 6) bias and misconduct, 7) discrimination, 8) the honorary author, 9) the ghost author, 10) the Cochrane collaboration, 11) open versus closed peer review.

Results: Peer review is a valuable tool in selecting scientific contributions to medical publications but will be subject to changes in the light of the advent of electronic publication and post publication peer review.

Conclusions: Increased awareness as to the evolution of peer review is mandatory.

\section{P-072}

Motor evoked potentials for monitoring cranial nerves during skull base surgery

\section{R Akagami*, C Dong, (Vancouver, BC)}

Background: Present monitoring techniques for cranial nerves do not allow the assessment of functional cranial nerve continuity during surgery. A novel monitoring technique is described and evaluated.

Methods: Motor evoked potentials for facial nerves during surgery for acoustic neuromas and other skull base tumors are reviewed. A nonrandomized control design is used to look at facial nerve outcomes before and after the implementation of the new technique. Facial nerve outcome is evaluated using the HouseBrackmann (H\&B) grade.

Results: Since implementation in October 2002, eight acoustic neuromas and one other basal lesion have been operated on to date (December 20). Further experience will be gained in the upcoming months. H\&B gr. 2 or better outcomes were obtained in $7 / 8$ patients. Grade 5 outcome was in a patient with a recurrent $5 \mathrm{~cm}$ neuroma. A patient with a giant chondrosarcoma had normal facial function postop.

Conclusions: Motor evoked potentials appear to be of benefit, improving surgeon comfort level and assisting in the extent of tumor resection. The same technique can also be used to monitor other motor cranial nerves and improve the safety of skull base surgery.

\section{Hypertrophic inflammatory neuropathy of the brachial plexus}

JL Steckley*, JM Findlay, AS Easton, C Hao, (Edmonton, AB)

Background: Hypertrophic inflammatory neuropathy (HIN) is a chronic inflammatory neuropathy. It is a rare disease and clinically presents as a peripheral nerve sheath tumor. Therefore, it is important to recognize this entity.

Methods and Results: A 46-year-old woman presented with a one-year history of progressive tender swelling of the left sternocleidomastoid region, with weakness and paresthesiae of the left upper extremity. Examination showed marked weakness of the left deltoid and biceps brachii muscles and a reduced biceps reflex. Compression of a palpable mass in the left sternocleidomastoid region produced shooting pain into the left upper extremity. EMG showed evidence of denervation of the left deltoid and biceps brachii muscles. CT showed a noncontrast-enhancing oval-shaped mass lesion in the region of the left brachial plexus. Clinical impression was nerve sheath tumor. At exploratory surgery, this mass appeared as a yellow, mucinous, plexiform nodular swelling of the superior and middle trunks. Frozen-section of the biopsy showed endoneurial edema, fibrosis and lymphocyte infiltration. Permanent sections revealed onion bulb formations with cells positive for S-100 and negative for EMAantibody. Variable sized lymphoid follicles were present in the endoneurium and perineurium. Pathologic diagnosis was HIN and, therefore, the patient was treated with antiinflammatory drugs instead of surgical removal of the tumor-like lesion.

Conclusions: HIN clinically mimics nerve sheath tumors. However, pathologic features differentiate it from nerve sheath tumors and pathologic diagnosis is crucial for treatment.

\section{P-074}

Intracranial pressure monitoring in patients with suspected idiopathic intracranial hypertension without papilloedema

T Darsaut*, $M$ Wheatley, V Mehta, J McKean, (Edmonton, AB)

Objective: To study the correlation between intracranial pressure (ICP) and headache severity in patients with presumptive idiopathic intracranial hypertension (IIH) without papilloedema.

Methods: Four female patients with presumptive IIH without papilloedema had intraparenchymal Codman ICP monitors inserted in an attempt to confirm raised ICP. All patients had headache responsive to lumbar puncture, normal CSF composition, normal MRI, and normal ophthalmologic exams. Two of the patients had previously had lumbar peritoneal (LP) shunts inserted for IIH. Both patients with LP shunts had previous shunt revisions for intractable headaches. ICP measurements and headache severity were recorded hourly.

Results: In all four patients, the headaches persisted throughout the period of monitoring. None were found to have elevated ICP. Two patients had intracranial hypotension with ICP recordings below zero. Two patients had normal ICP.

Conclusions: In the setting of presumptive IIH without papilloedema, a period of ICPmonitoring should be performed prior to establishing the diagnosis, as some patients do not have elevated ICP. In patients with previous LPshunts and persistent headaches, a period of ICP monitoring is indicated to rule out intracranial 
hypotension. The diagnosis of IIH without papilloedema remains a controversial diagnosis.

\section{P-075}

\section{Primary respiratory alkalosis after brainstem surgery}

VMehta*, T Darseault, M Jacka, M Henry, M Gowrishankar, (Edmonton, $A B$ )

Background: Severe postoperative primary respiratory alkalosis following neurosurgery has never been reported.

Methods: An 18-year-old female presents with a six-month history of progressive nausea followed by a recent onset of diplopia. Intracranial imaging demonstrated two lesions, one within the pineal region and a metastatic deposit on the floor of the fourth ventricle. Both serum and cerebrospinal fluid levels of $\beta$-HCG and $\alpha$ fetoprotein were within normal levels.

Results: A biopsy of the metastatic deposit was positive for a germinoma. Postoperatively, the patient was awake but had some difficulty swallowing. Multiple arterial blood gases over the first twelve hours postoperatively demonstrated a progressive primary alkalosis in the presence of a normal respiratory rate. Her initial arterial blood gas on room air was 7.57/ $\mathrm{PO}_{2} 114 / \mathrm{PCO}_{2} 16 / \mathrm{H}+27$. Over the next four hours her alkalosis progressed; $7.65 / \mathrm{PO}_{2}$ $127 / \mathrm{PCO}_{2} 12 / \mathrm{H}+22$. She was electively intubated in order to normalize her acid-base status. Over the following 12 hours her acid base status improved with ventilation 7.48/ $\mathrm{PO}_{2} 109 / \mathrm{PCO}_{2} 26 / \mathrm{H}+$ 33. A MRI on postoperative day one did not reveal any new brainstem pathology. At one month postoperatively she was still alkalotic with a $\mathrm{pH}$ of 7.61 .

Conclusions: This case represents a unique complication for which the pathogenesis is not completely understood.

\section{P-076}

\section{Ruptured intracranial dermoid cyst}

F Maroun*, G Murray, G O'Brien, J Jacob, N Hache, J Barron, T Smith, (St John's, NL)

Background: Dermoid cyst is an uncommon intracranial lesion. Rupture of a dermoid cyst is a rare occurrence. Fatal outcome of rupture was previously considered to be likely; recent reports do not suggest such a prognosis.

Methods: Published cases are reviewed, together with our case.

Results: Headaches and seizures are common presenting symptoms of ruptured dermoid cysts; also transient motor and sensory deficit, and chemical meningitis.

Conclusions: Surgical removal, rinsing of subarachnoid space following ruptured dermoid cyst is recommended. It is also advisable to operate on nonruptured cysts particularly when they are adjacent to basal blood vessels.

\section{P-077}

\section{Spontaneous regression of an AVM}

\section{R Reid*, I Fleetwood, (Halifax, NS)}

This case report describes a 63-year-old gentleman who presented with a generalized tonic clonic seizure and visual disturbance. Radiological investigations found him to have a left occipital Spetzler-Martin grade 2 AVM.
The patient was followed for approximately two years and during MRI image guidance preparation for his AVM resection a significant change was noted in the left occipital region containing the AVM. Further questioning of the patient did not reveal any new neurological symptoms in keeping with intracerebral hemorrhage or thrombosis of the AVM. Follow-up cerebral angiograms revealed spontaneous regression of the AVM.

Spontaneous regression of AV malformations is a relatively rare phenomenon and approximately only 35 other cases have been described in the literature. The exact reason for asymptomatic regression of an AVM is not fully understood, but is felt to be due to either thrombosis of the dominant draining veins or a decrease in afferent arterial flow.

\section{P-078}

\section{Intracranial hemorrhage complicating venous angiomas in} children

F Maroun*, G Murray, J Jacob, B Cramer, E Walsh, (St John's, $N L)$

Background: The natural history of venous angiomas is not clear; in particular, risk of intracranial bleed is unknown.

Methods: The history and clinical findings of a four-year-old girl and a seven-year-old boy are presented. Massive hemorrhage occurred in both of these following rupture of seemingly benign venous angiomas. Pertinent literature is reviewed.

Results and Conclusions: Even though venous angioma is thought to represent anomalous but competent intracranial venous circulation and excision of such lesion is not usually recommended: since catastrophic intracranial bleeding can occur, (as in our cases) therapeutic intervention is a consideration in management.

\section{P-079}

Stereotactic brain biopsy as an outpatient procedure: is routine CT scanning necessary?

\section{GE Pickett*, JF Megyesi, AG Parrent, (London, ON)}

Background: Following stereotactic brain biopsy (SBB), patients have traditionally been admitted. For outpatient SBB, head CT scanning has typically been required prior to discharge. The value of routine scans is uncertain, since most hemorrhages are asymptomatic. At our institution, SBB is performed with or without inpatient admission, and postoperative scans are not required.

Methods: We retrospectively reviewed hospital records of all adult patients who underwent SBB on a same-day-admit or one-daycare basis at our institution over a three-year period.

Results: Of 83 patients who met inclusion criteria, 37 were treated as outpatients. Postoperative CT scanning was performed in only eight outpatients prior to discharge. There was one case of avoidable morbidity among the outpatients: a patient returned following a seizure the evening of the procedure, and CT scanning demonstrated a small epidural hematoma overlying the biopsy site. Conversely, 38 of the 46 inpatients experienced no morbidity and represented avoidable admissions. The remaining eight patients displayed symptoms during observation in the recovery room. There were no cases of delayed in-hospital morbidity.

Conclusions: SBB may safely be performed as an outpatient procedure, and routine CT scanning is not necessary prior to 
discharge. Overnight inpatient observation did not detect any late morbidity.

\section{P-080}

\section{Effect of new anti-angiogenic compound on human astrocytomas}

\section{T James*, B Qian, G Zadeh, A Guha, (Toronto, ON)}

Introduction: Glioblastoma multiforme (GBM) are one of the most highly vascularized tumors, and vascular endothelial growth factor (VEGF) and platelet derived growth factor (PDGF) are two of the key factors in promotion of angiogenesis in GBMs. SU11248, is an anti-angiogenic compound produced by Sugen Pharmaceuticals, which has been shown to prevent phosphorylation of VEGF receptor Flk-1/KDR and the PDGF receptor PDGFRb. We postulate that it can disrupt angiogenesis and thereby arrest or decrease the growth of GBMs.

Methods: We studied the effect of SU11248 on growth and vascularity of mouse xenograft models of human GBMs. Drug was administered daily for 15 days orally in twenty mice with five mice as control, receiving vehicle only, and five mice receiving no treatment. Tumor volume was calculated daily together with body weight. Animals were sacrificed at the end of drug delivery and analyzed for extent of tumor vascularity.

Results: Our preliminary results demonstrate a decrease in growth rate of xenografts associated with restricted tumor vascularity.

Conclusions: Inhibition of VEGF and PDGF receptor can act as a potential therapeutic strategy in human astrocytomas. As future work we will be focusing on assessing the overall effect of SU11248 on endothelial cell proliferation and mechanism of drug action.

\section{P-081}

\section{Unusual indications for spinal cord stimulation}

\section{Berk*, I Beauprie, R Brownstone, (Halifax, NS)}

Background: Spinal cord stimulation (SCS) is safe and effective for the treatment of chronic pain. The success of SCS depends on the diagnosis of a responsive pain syndrome and the use of strict criteria for patient selection. We are presenting two unusual cases of chronic pain, successfully treated with spinal cord stimulation.

Methods: Case \#1: This 53-year-old lady was suffering from complex regional pain syndrome following a radical mastectomy for breast cancer. Her pain was in the anterior chest radiating to her left shoulder, around the back to the midline as well as down the left arm. She had a trial of SCS followed by implantation of a permanent epidural lead at C7-T1 level. Case\#2: This 30-year-old man developed pancreatitis and chronic pain secondary to crush injury after a motor vehicle accident. His pain was in his abdomen radiating to his back and the left shoulder tip. He had a trial of SCS followed by implantation of a permanent epidural lead at T3 level.

Results: Once the optimal parameters for SCS were achieved, the need for analgesics was reduced. In the second patient, the pain was reduced not only in the area covered by the stimulation (abdomen) but also in the area of referred pain (shoulder tip). The ADL and quality of life scores in SF-36 scale were significantly improved.

Conclusions: SCS may be an effective treatment alternative for pain of visceral and malignant origin in selective cases.
P-082

\section{An interim report on the PROLONG outcomes registry}

$J$ Yazdi*, (Toledo, OH) and The PROLONG Study Group,

(Baltimore, $M D$ )

Background: No single treatment for malignant gliomas has proven to be a home run. Therefore, multimodality treatment should be the mainstay of therapy.

Methods: A prospective registry study, PROLONG, was initiated to follow patients receiving Gliadel Wafer along with other therapies as judged by their physician. This is a preliminary report of the current findings.

Results: Enrollment began on 12/13/01 and as of 12/31/02, 171 patients have been enrolled. Their diagnoses include: GBM, AA, AOA, AA, metastatic, and other. Median age is 59, median KPS is 90 (range 20-100). After one year of enrollment, there are 144 patients with malignant gliomas enrolled. Follow-up is available on 101 of these patients. Current survival in the primary surgery setting ranges from 57-374 days and from 46-325 days for recurrent surgery patients. Thirty patients have died to date, five of whom died in the first thirty days and four of whom were recurrent surgery patients who survived longer than six months. Adjunct therapies include IV BCNU, temozolomide, CPT-11, thalidomide and tamoxifen.

Conclusions: Gliadel Wafer is a viable option for the treatment of malignant gliomas and in combination with other therapies because of its lack of systemic side effects. Future reports will include additional survival analysis.

\section{P-083}

Increasing the level of neuroprotection and quality of neurointensive care: bedside CT scanning of the head

T Gunnarsson*, (Toronto, ON); A Theodorsson, J Hillman, (Linkoping, Sweden)

Background: To avoid unnecessary intrahospital transports we have performed bedside CT scans of the head in the neurointensive care unit (NICU), University Hospital, Linkoping, Sweden, for the last six years.

Methods: By combining new and old prospective data we estimated the number of intrahospital transports and complications that have been avoided during the first five years using this technique. We also estimated the number of minutes the patients have gained in the NICU as well as the time gained by the nursing staff on patient care.

Results: In five years we have avoided at least 1200 intrahospital transports and 268 complications associated with it. The number of minutes gained for the patients in the controlled environment of the NICU amount to 79248. The nursing staff spent 180544 more minutes in the NICU which is the time a nurse devotes to patient care in two years.

Conclusions: By avoiding intrahospital transports of patients from the NICU we have prevented one complication a week, increased the time the patients stay in this controlled environment and the nursing staff has devoted more time to patient care. This way we have increased the level of neuroprotection and improved the care of our critically ill patients. 


\section{P-084}

Recombinant tissue plasminogen activator in the treatment of intraventricular hemorrhage secondary to periventricular arteriovenous malformation prior to surgery: a case report

K Kumar, A Verma, (Regina, SK); D Demeria*, G Hunter, (Saskatoon, SK)

Background: Intraventricular hemorrhage (IVH) is known to cause acute obstructive hydrocephalus; refractory elevated intracranial pressures (ICP), lowered cerebral perfusion pressures (CPP) leading to cortical ischaemia. Frequent obstruction of external ventricular drains (EVD) due to thrombus is a recurring theme.

Methods: We present a case of an 11-year-old male with acute onset of headache, followed by two seizures, loss of consciousness, decerebration, right temporal hematoma, IVH and acute obstructive hydrocephalus. The IVH was secondary to periventricular arteriovenous malformation (AVM), which was not visible on admission angiography, and treated by intraventricular infusion of $r$ tPA, preceding surgical intervention.

Results: A right EVD was placed, but functioned poorly. ICP could not be controlled using conventional methods. Five mg of rtPA was injected into the ventricular system, through the EVD. This was repeated daily for four days. This resulted in progressive improvement in ICP and clinical status. Once the clot partially cleared, MRI and MRA suggested the presence of right periventricular AVM, which was confirmed by angiography, and subsequently resected.

Conclusions: r-tPA is effective in resolving IVH causing obstructive hydrocephalus, uncontrollable ICP posing a lifethreatening situation, secondary to ruptured AVM, prior to surgical intervention.

\section{P-085}

\section{Trigeminal neuralgia nomenclature}

\section{J Rempel*, AM Kaufmann, (Winnipeg, MB)}

The nomenclature of trigeminal neuralgia (TN) includes many synonyms and inconsistently defined adjectives such as "idiopathic", "essential" and "atypical" that do not necessarily represent the clinical features or disease etiology. These factors may confuse analysis and comparison of treatment results. We defined categories of TN and assessed the outcome of microvascular decompression (MVD) surgery.

Four categories of TN include: typical (as per IHS criteria), advanced (with associated aching/burning attacks), atypical (with prominent constant pain) and tumor-related (without neuropathy features). Surgical observations of neurovascular compression were recorded and annual outcome results obtained. $\mathrm{TN}$ cases due to multiple sclerosis (MS) were excluded.

The senior author performed 163 original MVD surgeries for TN between 1996 and 2002 (100 typical; 33 advanced; 28 atypical and two tumor-related). Neurovascular compression of the trigeminal root entry zone was identified in all cases, with superior cerebellar artery the primary culprit in $75 \%$. Outcome was excellent and good more frequently with typical TN (70\% \& 15\%) compared to atypical $\mathrm{TN}(63 \% \& 7 \%)$, although not reaching statistical significance $(\mathrm{p}=.17)$.

Neurovascular compression is the common etiology of typical, advanced, atypical and tumor-related TN. Therefore the terms "essential TN" or "idiopathic TN" should be abandoned. Clinical categories of TN should be defined and studied as potential predictors of outcome for various TN interventions.

\section{P-086}

\section{Double craniotomy approach}

\section{AM Kaufmann*, $M$ West, (Winnipeg, MB)}

Introduction: A variety of cranial base approaches have been developed to treat neuropathologies that traverse the supra/infra tentorial compartments. We describe our experience with simultaneous retrosigmoid and frontal-temporal craniotomies.

Methods: We recently utilized a novel "double crani" technique to treat five patients with large petroclival meningiomas extending into the supratentorial spaces. The same approach was used to treat two other patients with concurrent posterior fossa and supratentorial cerebrovascular pathologies.

Results: All patients were positioned lateral decubitus. The standard retrosigmoid and frontal-temporal approaches were performed simultaneously, but independently through separate incisions. A single scrub nurse attended to both fields. The two neurosurgeons used separate coagulation units, drills, ultrasonic aspirators and microscopes. The working trajectory of the two approaches did not create conflict and did not impair the use of intraoperative monitoring. At later stages of tumor resection, the deep surgical fields merged, facilitating cooperative maneuvers through the different approaches. The technique allowed visualization of cranial nerves 2-12 and both the carotid/vertebro-basilar vasculature.

Conclusions: We favored this "double crani" technique over a combined supra/infra tentorial craniotomy or staged procedures. The surgical time was reduced, without compromise of surgical effectiveness or safety.

\section{P-087}

Infection rates in standard vs. hydrogel coated ventricular catheters: a prospective randomized clinical trial

AM Kaufmann*, M Kozey-Rogers, (Winnipeg, MB); T Lye, $M$ Hamilton, (Calgary, AB); G Redekop, (Vancouver, BC)

Background: A novel hydrogel-coated catheter, designed to reduce bacterial colonization and infection was compared to standard silastic external ventricular drain (EVD) catheters in a prospective randomized clinical trial.

Methods: Neurosurgery patients receiving EVD were randomized to either catheter type. Daily cerebrospinal fluid (CSF) samples were collected, and infection was defined as heavy growth in any single sample or light growth in two consecutive samples. A secondary analysis was also conducted for "probable" CSF infection, including patients started on antibiotics after light growth in a single CSF sample. Statistical analyses included Kaplan-Meier survival curve estimates accompanied by Log Rank and Breslow tests.

Results: Two hundred and twenty-nine patients were randomized, and 158 were available for comparison. Reasons for post-randomization exclusion included: EVD use was less than 48 hours (35), open head injury (10), incomplete CSF laboratory data (17) and other protocol violations (9). The two study groups had 
similar clinical characteristics including average duration of EVD use (8 4 days). Definite CSF infection occurred in seven of 158 patients, and probable infection in another six (9\% total). There was no difference in incidence of CSF infection between the two EVD types.

Conclusions: Infection remains a common hazard in the use of EVD, and we found no reduction of infection using the hydrogelcoated catheters.

\section{Movement Disorders}

\section{P-088}

\section{Prevalence of spontaneous oral dyskinesia in the elderly: a reappraisal}

P Blanchet*, O Abdillahi, C Beauvais, P Rompre, G Lavigne, (Montreal, $Q C$ )

Background: The prevalence and place of spontaneous oral dyskinesia (SOD) as a nosologic entity remain controversial. The apparent similarity between SOD and tardive dyskinesia (TD) is used as an argument to minimise the prevalence of TD and causal role of antipsychotic drugs.

Methods: In this cross-sectional field study, we observed a general elderly population attending day care centres to document the prevalence and phenomenology of SOD. A survey covering medical and dental issues was filled by volunteers.

Results: 1029 subjects at least 60 years of age were observed: 38 were suspected to have SOD (prevalence rate of $3.7 \%$ ). The survey was completed by 542 subjects, including 57 with probable TD. In surveyed subjects without TD, the proportion of women and men $(8.3 \%$ vs. $6.6 \%)$ and of edentulous and dentulous subjects $(7.3 \%$ vs. $6.4 \%)$ displaying SOD was comparable. More frequent ill-fitting dentures $(\mathrm{p}=0.005, \mathrm{OR}=3.2)$, oral pain $(\mathrm{p}=0.01, \mathrm{OR}=3.1)$ and $\mathrm{a}$ lower rate of perception of good oral health $(\mathrm{p}=0.04, \mathrm{OR}=0.37)$ distinguished SOD subjects from nondyskinetics, but variables including age, edentulousness, use of dentures, mouth dryness or chewing difficulties, were similar. Individuals with SOD displayed a less complex repertoire of visible stereotyped movements than probable TD cases.

Conclusions: SOD is characterized by a much lower prevalence rate and less complex stereotyped manifestations than TD, and appears more strongly associated with ill-fitting dentures than edentulousness. Further studies are needed to clarify the role of various determinants of poor oral health in its etiopathogenesis.

\section{P-089}

\section{Low dose of topiramate is effective on cervical tremor}

Emmanuelle Pourcher*, Marie-Claude Fortin, Amelie Beausoleil, (Quebec, QC)

Background: Cervical tremor with mild dystonic features, classified as possible essential tremor by the TRIG and National Institute of Health (NIH) criteria is a frequently encountered condition, especially in elderly women either isolated or in association with hand or voice tremor. Botulinum toxin has been proposed for this type of tremor in which propanolol and primidone have a low therapeutic index. We found a superior efficacy of topiramate in open label, dose finding, tolerance exploring trials confirmed in the following double blind randomized, placebo controlled, cross-over study.

Design: Population: 20 patients, age 65.06 (range: 57-81), gender masculine/feminine 12/8, average disease duration 16 years, (range: $3-25)$, positive family history per gender ( $85 \%$ masculine, $41 \%$ feminine), isolated cervical localization ( $25 \%$ masculine, $45 \%$ feminine) associated cerebro-vascular risk factor (75\% masculine, $50 \%$ feminine). Undistinguishable capsules containing either saccharine placebo or topiramate BID were randomly distributed for four months with an ascending titration of $25 \mathrm{mg}$ every two weeks from $25 \mathrm{mg}$ HS to $25 \mathrm{mg}$ twice BID and a descending titration to 25 $\mathrm{mg}$ of topiramate BID for two weeks when switched to placebo.

Results: Statistical comparisons of changes in the severity of head tremor after two months of treatment favoured topiramate against placebo ( $71 \% 6$ improvement versus $23 \% 10$, p significant at $0.05)$. No distinction in improvement was found between isolated vs non isolated head tremor, positive vs negative family history. Although significantly improved, hand tremor was less impressively controlled (57\% 15 versus $27 \%$ 5). Tolerance was good, although most female patients accused minor transient lingual and hand paresthesia. One female patient abruptly stopped medication due to a postcoital transient global amnesia observed at $75 \mathrm{mg}$ a day of topiramate, a probably unrelated side-effect. Two patients needed the addition of a low dose of SSRI to control a drug-related dysphoria.

Conclusion: Low-dose, slow-titration of topiramate reveals benefit in a sub-type of essential tremor often found drug-resistant.

\section{P-090}

Intracerebral administration of liposomal tacrolimus prevents graft rejection and increases cell survival in hemiparkinsonian rats

\section{A Alemdar*, D Sadi, I Mendez, (Halifax, NS)}

Background: Systemic administration of immunosuppressants can often lead to toxic side effects. The purpose of the present study was to investigate whether it is possible to increase neural graft survival without the need for daily immunosuppressant injections, by co-transplanting either liposomal tacrolimus (LTAC), liposomal rapamycin (LRAPA), or a combination of the two drugs, LRAPATAC, along with fetal mouse ventral mesencephalic cells in the hemiparkinsonian rat.

Methods: Rats with unilateral 6-hydroxydopamine lesions were transplanted with 800,000 fetal mouse ventral mesencephalic cells and randomly divided to one of four groups, receiving the cells only, a cell suspension containing $0.68 \mathrm{mM}$ LRAPA, a cell suspension containing $2.0 \mathrm{mM}$ LTAC, or a cell suspension with LRAPATAC containing both $0.68 \mathrm{mM}$ RAPA and $2.0 \mathrm{mM}$ TAC. Transplanted animals were assessed for amphetamine-induced rotational behavior three and six weeks post-transplantation. Cell survival was assessed using tyrosine hydroxylase (TH) immunohistochemistry, while graft rejection was assessed by major histocompatibility complex class I and II (MHC I and II) staining.

Results: A significant reduction in rotational scores at six weeks post-transplantation was seen only in the group receiving LTAC. As well, there were significantly more surviving THir cells in the LTAC group and significantly less MHC I and II staining.

Conclusions: This study demonstrates the feasibility of using a 
liposomal formulation of immunosuppressant directly in the brain to prevent graft rejection and circumvent the need for systemic immunosuppression and its potential side effects. The results of this study may have important implications in the optimization of clinical neural transplantation for Parkinson's disease.

\section{P-091}

\section{Reducing hardware related complications of deep brain stimulation}

C Constantoyannis*, C Honey, (Vancouver, BC); C Berk, R Brownstone, I Mendez, (Halifax, NS)

Background: Deep brain stimulation (DBS) is increasingly used for movement disorders and pain. DBS introduces a new series of problems related to its hardware. This study reviews these potential complications and highlights methods of reducing them.

Methods: A prospective study of all DBS operations performed in Vancouver and Halifax was conducted from January 1999 to January 2003. One hundred and forty-four patients underwent DBS surgery for movement disorders or pain and were included in the study. Hardware related complications such as infection and lead malfunction, migration or fracture were recorded.

Background: The mean follow-up was 22 months. One hundred and forty-four patients had 204 electrodes implanted. There were 13 hardware related complications, with an infection rate per electrode of 4.4 percent, erosion rate per electrode of 1 percent, fracture rate of 1.4 percent and an overall adverse effect per patient of 9 percent. There was a significantly higher risk of infection in patients who underwent staged procedures with temporary externalization of the electrode. Our analysis showed that the risk of infection was increased four-fold in patients who had straight scalp incision compared to those with a curvilinear incision.

Conclusions: Hardware related complications were not common. Two variables were associated with an increased infection rate (externalization and straight scalp incision). The authors highlight important operative techniques to reduce complications during DBS surgery.

\section{Multiple ScLerosis}

\section{P-092}

Acute disseminated encephalomyelitis: evidence for diffuse abnormality involving the normal appearing brain tissue

SD Brass*, AC Santos, S Narayanan, A Bar-Or, DLArnold, (Montreal, $Q C$ )

Background: The MRI findings in acute disseminated encephalomyelitis (ADEM) may be difficult to differentiate from multiple sclerosis (MS). Previous studies using magnetization transfer imaging (MTI) (Inglese, et al. Neurology 2001;56:A256) and magnetic resonance spectroscopy (MRS) (Bizzi, et al. AJNR 2001;22:1125-1130) in ADEM have reported that the pathology is confined to macroscopic lesions and suggested that this could be useful in discerning MS from ADEM. We used MRI, MTI and MRS to assess the extent of abnormality of brain tissue that appeared normal on conventional MRI in a patient with ADEM.

Methods: A 50-year-old female in the acute phase of ADEM and three female age-matched healthy volunteers [mean \pm standard deviation $(\mathrm{SD})=51.9 \pm 8.8$ years] underwent conventional MRI, MTI and MRS.

Results: The neuronal marker compound, N-acetylaspartate/ creatine (NAA/Cr) over the entire VOI was more than 6 standard deviations below that of healthy controls ( 2.25 vs $3.12 \pm 0.14)$. NAA/Cr in voxels containing $100 \%$ lesion was 2.8 SD below control values $(1.71$ vs $2.77 \pm 0.38)$. In voxels containing $100 \%$ normal appearing white matter (NAWM) it was 2.6 SD below controls (2.61 vs $3.71 \pm 0.42$ ) and in voxels containing $100 \%$ normal appearing gray matter (NAGM) it was 3.5 SD below controls (2.22 vs $2.60 \pm$ 0.11 ). In each of these same voxels (lesion, NAWM, NAGM), choline/creatine $(\mathrm{Cho} / \mathrm{Cr})$ was elevated $(>2 \mathrm{SD}$ above the control mean) in the patient relative to controls and $\mathrm{Cho} / \mathrm{Cr}$ was also elevated in the entire VOI. Mean MTR in the NAWM (33.47) was 2 SD lower in our patient than in controls $(35.88 \pm 0.26)$ but was abnormal in the NAGM (24.69 vs $25.08 \pm 0.34)$. Mean MTR was lower within lesions (20.96).

Conclusions: This report demonstrates that the pathology of ADEM is not necessarily limited to the macroscopic lesions visible on MRI but as in the case of MS, can be present diffusely throughout the NAWM and NAGM.

\section{P-093}

Meningoencephalitis temporally related to initiation of interferon $\beta$-1a in a patient with multiple sclerosis

MB Cossoy*, R McKelvey, RJB Macaulay, (Halifax, NS); A Bar-Or, (Montreal, $Q C$ )

Background: Interferon $\beta$-1a (If $\beta 1$ a) is an accepted treatment for relapsing-remitting multiple sclerosis (MS), and may also benefit patients with secondary progressive MS who continue to have relapses. It is usually initiated at $20 \%$ of the target dose for two weeks to allow tachyphylaxis to develop. It often causes a flu-like reaction in patients, attributed to cytokine induction, but has not previously been associated with aseptic meningoencephalitis.

Clinical Case: A 65-year-old woman suffering from secondary progressive MS with clinical relapses received a single 22microgram dose of If $\beta 1$ instead of the planned $20 \%$ dose. She became acutely ill within two hours, was hospitalized and started on intravenous methylprednisolone. She recovered over the next two days, but developed a deep venous thrombosis of her leg and succumbed to a presumed pulmonary embolism fifteen days after admission.

Results: Neuropathologic autopsy confirmed the diagnosis of MS as well as acute hypoxic/ischaemic injury attributed to respiratory failure. It also showed a resolving meningoencephalitis with patchy perivascular lymphocytic cuffing in the leptomeninges and cerebral white matter.

Conclusions: Meningoencephalitis is not characteristic of MS or hypoxic-ischaemic encephalopathy. It may reflect the single exposure to a 22-microgram dose of If $\beta 1$ a. This adverse effect has not previously been reported, but the pathologic changes accompanying flu-like reactions to If $\beta 1$ a have not been well established. 


\section{P-094}

\section{The prevalence and significance of pain in multiple sclerosis}

L Kalia*, P O'Connor, (Toronto, ON)

Background: Pain is an underappreciated symptom in MS. Given the importance of pain for patients with other chronic conditions (e.g. arthritis), it is important to study pain in MS. The purpose of this study was to assess potential relationships between pain, gender, disability, mood, and health-related quality of life in MS.

Methods: 99 MS patients participated by completing the SF-36, Hospital Anxiety and Depression Scale, and Short-Form McGill Pain Questionnaire.

Results: Point prevalence of pain in MS was $26.3 \%$. For males, SF-36 bodily pain (BP) scores were not significantly different between MS patients and Canadian norms $(\mathrm{p}=0.99)$. However, mean BP scores for females with MS was 56.2 versus 72.3 for normative Canadian samples $(p=0.0001)$. BP scores were not significantly different between MS patients and osteoarthritis patients $(\mathrm{p}=0.40)$. Pain in MS had no significant relationship to age, disease subtype or duration. Pain was more strongly correlated with measures of mental health ( $\mathrm{r} 0.44, \mathrm{p}=0.0001)$ versus measures of disability $(\mathrm{r}-0.11$, $\mathrm{p}=0.29$ ). For females but not males with MS, pain was significantly related to mood $(\mathrm{r}-0.53, \mathrm{p}=0.0001$ and $\mathrm{r}-0.24, \mathrm{p}=0.14$, respectively).

Conclusions: Pain is common in MS with an average intensity that does not differ significantly from that of arthritis. Presence of pain is not related to disability, age, disease subtype or duration but is strongly associated with measures of mental well-being.

\section{P-095}

\section{Communicable respiratory disorders and the risk of multiple sclerosis in Alberta, Canada}

$S$ Warren*, L Svenson, KG Warren, (Edmonton, $A B$ )

Background: Researchers have speculated that multiple sclerosis (MS) may be caused by a communicable infection. MS is more common in temperate than tropical climates, and there is evidence that the frequency of respiratory infections increases with distance from the equator. However, there appear to be no published studies that correlate the frequency of MS with communicable respiratory disorders (CRDs).

Methods: Alberta Health Insurance Plan data were used to calculate the incidence of MS and CRDs (acute respiratory infections, upper respiratory tract infections, bronchitis, influenza, viral pneumonia, and other pneumonia) for all 17 regional health authorities (RHAs). Pearson correlation coefficients were used to analyze the data.

Results: The only positive correlation was between MS and bronchitis at +.36 . While the size of this correlation is notable by common standards, it is not statistically significant at $\alpha=.05$. There was no notable correlation between MS and acute infections. All other correlations were notable but negative and not statistically significant: upper tract -.42 , influenza -.33 , viral pneumonia -.32 and other pneumonia -.37 .

Conclusions: With only 17 RHAs and low variability in the frequency of both MS and CRDs, the statistical power of this study was limited. Nevertheless, the size of the positive correlation between MS and bronchitis may warrant further descriptive studies in geographic locations which overcome these limitations.
P-096

\section{Do serum levels of prolactin correlate with age in multiple sclerosis?}

Ali Abdollahi*, Reza Tabasi, Jamshid Javaheri, (Tehran, Tehran)

Purpose: As regards to conflicting results of previous studies performing to serum prolactin alterations in MS, we attempted to elucidate other aspect of this ongoing issue to determine if serum prolactin level can be considered as a prognostic factor.

Methods and Participants: 25 patients with signs, symptoms and MRI findings compatible with multiple sclerosis were enrolled and their demographic data including, sex, age, age at the onset of the disease and also duration of the disease were obtained. Serum prolactin levels were measured.

Results: Most patients were 16-20-year-olds at the onset of the disease. We didn't find any statistically important difference between serum prolactin levels of the patients and age matched controls $(10.30 \mathrm{ng} / \mathrm{ml}$ vs. $10.10 \mathrm{ng} / \mathrm{ml}$ with $\mathrm{P}=0.001)$. Also, we couldn't correlate serum prolactin levels with ages of the patients. Only two patients $(8.7 \%)$ had elevated serum prolactin $(22.40 \mathrm{ng} / \mathrm{ml}$ and $25.7 \mathrm{ng} / \mathrm{ml}$ ) in whom MRI findings revealed no hypothalamopituitary lesions.

Conclusions: Serum prolactin levels don't seem to have any correlation with age at the onset of the multiple sclerosis.

\section{NEURO-ONCOLOGY}

\section{P-097}

\section{Giant cell ependymoma of the spinal cord}

D Fourney*, (Saskatoon, SK); A Siadati, J Brunner, L Rhines, (Houston, TX); Z Gokaslan, (Baltimore, MD)

Background: The morphological features and biological behavior of ependymomas may vary considerably. In recent years, a rare variant composed mainly of pleomorphic giant cells has been reported: two cases involved the filum terminale, and two were located in the cerebral hemispheres.

Methods: We report, to our knowledge, the first case of a giant cell ependymoma arising from the spinal cord. The patient was originally diagnosed with cervical spinal cord astrocytoma after a limited biopsy performed at another institution. More abundant tissue obtained at gross-total resection included areas of welldifferentiated ependymoma.

Results: The histological features of the tumor were extremely unusual, with a major component of pleomorphic giant cells. However, histological, immunohistochemical, and electron microscopic features were consistent with ependymoma.

Conclusions: This case is of clinical significance because it underscores the importance of obtaining a sufficient amount of tissue for an adequate pathological diagnosis, especially at the time of frozen section, when the decision to biopsy the tumor or attempt a complete resection is in question. Secondly, it shows how easily the giant cells of this rare ependymoma variant can be mistaken for gemistocytes (i.e., anaplastic astrocytoma) in the absence of perivascular pseudorosette formation. The distinction has clinical implications because careful microdissection of spinal cord ependymomas often distinguishes a cleavage plane that makes these tumors amenable to complete resection. 


\section{P-098}

Schwannoma of the oculomotor nerve presenting with atypical facial pain

C Spivak*, R Griebel, C Robinson, V Chow, (Saskatoon, SK)

Background: Schwannomas of the occulomotor nerve are very rare tumors; only 30 cases have been reported. They usually present with a third nerve palsy. Only one of these cases presented with oculomotor palsy and facial pain.

Methods: Retrospective chart review.

Results: We report a case of a 52-year-old female who presented with left-sided atypical facial pain without a third nerve palsy. She was neurologically intact and did not have evidence of neurofibromatosis. MR imaging revealed a large left mesial temporal lobe mass. It was found to be originating from the left third cranial nerve. It was completely resected and the third nerve was left intact. Postoperatively the patient had complete left third nerve palsy. The tumor was found to be a schwannoma. No recurrence was found after two years of follow-up.

Conclusions: Occulomotor schwannoma may present with atypical facial pain. Complete resection is possible, but is often complicated by third nerve palsy.

\section{P-099}

\section{Metastases from pituitary adenomas}

A Sabbagh*, R Leblanc, M-C Guiot, D Melanson, (Montreal, QC)

Background: We report the case of a patient with a pituitary macro-adenoma that seeded to the orbito-frontal region as a separate, secondary tumor three years following the transcranial resection of the original adenoma.

Methods: Our case and all similar cases previously reported were analyzed for pertinent epidemiological and biological features.

Results: Ninety-two cases were available for review. Males were equally affected as females. Sixty percent of the adenomas were secreting, $40 \%$ were hormonally inactive, and $40 \%$ of the primary tumors had atypical histological features. The interval from initial diagnosis to the appearance of the metastases ranged from 0-20 years (mean: 6.1 years). The metastases were intracranial $(43 \%)$, intraspinal (16\%), and systemic (42\%), the latter involving the liver, lymph nodes, skeleton and lungs.

Conclusions: Metastasis from a pituitary adenoma is probably more frequent than commonly appreciated. Reports of this occurrence have increased with advances in neuro-imaging: $72 \%$ of cases were reported following the advent of computed tomography. The primary tumor need not exhibit aggressive histological features. The metastatic cells can seed through the subarachnoid space or spread via the lymphatic or vascular systems, perhaps reflecting the particularities of the vasculature of the pituitary gland.

\section{P-100}

\section{Astrocyte specific knockout of tumor suppress or PTEN/MMAC 1 in transgenic mouse model}

$Q$ Wei*, J Levoie, D Kaplan, A Guha, (Toronto, ON)

Introduction: Loss of the tumor suppressor gene, PTEN/MMAC1, is common in malignant human astrocytomas. We have taken two strategies to knockout PTEN/MMAC1 specifically in embryonic and adult astrocytes.

Methods: First, to knockout PTEN in adult astrocytes, we are developing adenovirus to express Cre-recombinase specifically in astrocytes. Cre-recombinase expression has been detected in vitro and the function will be tested in Z/AP reporter mice before the adenovirus being stereotactically injected into PTEN flox/flox mice. The second strategy is to develop inducible astrocyte specific Crerecombinase mice, which will be bred to the PTEN flox/flox mice with the tamoxifen inducible Cre-recombinase (Cre-ER) resulting in excision of PTEN in adult astrocytes.

Results: We have already transfected embryonic stem cells (ES) from reporter Z/AP mice, with a GFAP:Cre-ER construct. In vitro test of these ES cells for GFAP regulation and tamoxifen induction of Cre-recombinase have shown the nuclear localization of Cre expression and Cre-recombinase activity. Recently a new construct, GFAP-CRE-ERT2, predicted more sensitive to tamoxifen induction, has been transfected to Z/APES cells. The in vitro test is undertaken. Conclusions: These strategies will allow us to understand the role of PTEN/MMAC1 in glioma genesis independently and in the context of other genetic alterations.

\section{P-101}

Clinical studies of photodynamic therapy formalignant brain tumors: facial nerve palsy after temporal fossa photoillumination

P Muller*, B Wilson, L Lilge, V Yang, A Varma, A Bogaars, (Toronto, ON)

Background: In two randomized prospective multicentered studies of brain tumor PDT, more than 180 patients have been accrued.

Methods and Results: At the Toronto Site [80 patients] we recognized two patients who developed a lower motor neuron [LMN] facial paralysis in the week following PDT treatment. Both patients had a temporal lobectomy and the residual tumor cavity was photo-illuminated. The surface illuminated included the temporal fossa floor thus potentially exposing the facial nerve to the effect of PDT. The number of frontal, temporal, parietal, and occipital tumors in this cohort was 39,24, 12 and 4, respectively. Of the 24 temporal tumors 18 were randomized to Photofrin-PDTand received either 40 or $120 \mathrm{~J} / \mathrm{cm}^{2}$. Of these 18 , a temporal lobectomy was carried out in 10 exposing the middle fossa floor as part of the tumor resection. In two of the 10 patients a postoperative facial palsy occurred. Both patients recovered facial nerve function in six and 12 weeks, respectively. $46 \mathrm{~J} / \mathrm{cm}^{2}$ were used in the former and $130 \mathrm{~J} / \mathrm{cm}^{2}$ in the latter.

Conclusions: Among more than 700 supratentorial brain tumor operations in the last decade involving all pathologies and all locations, no case of early postoperative LMN facial palsy was identified in the absence of PDT. Although it is possible that these two patients had incidental Bell's palsy, we now recommend shielding the temporal fossa floor during PDT. 


\section{P-102}

PI3Kinase and MAPKinase activation as prognostic indicators of glioblastoma multiforme

\section{G Zadeh*, N Sabha, P Shannon, A Guha, (Toronto, ON)}

Introduction: This study focuses on determining the prognostic value of two main signaling pathways (PI3Kinase and MAPKinase) that have been demonstrated to be involved in the growth of glioblastoma multiformes (GBM). Since GBMs are heterogeneous tumors, molecular characterization of the tumors will allow designing more effective and tailored treatment for individual tumors.

Methods: Twenty GBM samples from the Toronto Brain Tumor Bank were stained for molecular markers that are relevant to the above two mentioned signaling pathways in addition to western blot analysis for the same markers: MAPk, p-MAPK, EGFRvIII, PTEN, Akt and p-Akt. The extent of staining was determined by computer image analysis. Patient age, total survival, tumor proliferation index and vascularity was correlated to the expression of the above mentioned factors.

Results: For PTEN, MAPk or p-MAPk we found no correlation with patient prognosis. There was a direct correlation, however, between the extent of EGFRvIII and Akt stain.

Conclusions: This pilot study indicates that using present antibodies available for the various molecular markers thought to be relevant in GBMs only EGFRvIII thus far can be used as a prognostic indicator, with future work on a larger cohort of tumors looking closer at the other markers potentially identifying other tumors.

\section{P-103}

The ecology of CNS tumors in the province of Saskatchewan during the last three decades

Farhad Pirouzmand, Venkat Sadanand, Sudesh Ebenezer*, (Saskatoon, SK)

Background: The incidence of certain brain tumors (BT) is thought to be increasing in Saskatchewan. This study documents the epidemiology of BTs longitudinally over three decades in Saskatchewan.

Methods: Data on all the CNS tumors from 1970 to 2002 from the Brain Tumor Registry in Saskatchewan was analyzed. The data was divided into three time periods: group I 1970-1980, group II 1981-1991, and group III 1992-2002. The epidemiology of primary, metastatic, malignant, and benign brain tumors were analyzed. The univariate statistics was used in analyzing the distribution of incidence of tumors in different study groups, sex and geographic locations.

Results: The average annual incidence of BTs was estimated at 7.1 cases per 100,000 person-years. Males constituted $54.5 \%$ of all these tumors. Aggregate incidence frequency was bimodal with peaks at ages less than five years and about 65 years. The behavior was as follows: benign $13.9 \%$, invasive $81.2 \%$ and uncertain $4.8 \%$. The average annual incidence for each group was 5.4, 6.9 and 9.1, respectively. The incidence of certain tumors over the study period revealed an increase in CNS lymphoma $(\mathrm{p}<.05)$, reduction in meningioma and metastatic diseases $(\mathrm{p}<.05)$ and no statistically significant change in astrocytoma group.
Conclusions: The incidence of pathologically proven BTs in Saskatchewan is less than previously reported. The surprising reduction of meningiomas and surgically treated metastatic tumors requires further analytic studies to explore possible underlying causes for these findings.

\section{P-104}

Milrinone may prevent or reverse intra-operative neurological dysfunction during tumour surgery

RM Waldkircher de Oliveira*, RF Del Maestro, (Montreal, QC)

Background: Cerebral injury may occur during the resection of tumours under neuroleptic anaesthesia and be identified as a sudden or progressive decrease in neurological function. It was hypothesized that at times this injury may be secondary to operative induced vasospasm and/or vessel injury and that the cerebral vasodilator Milrinone may prevent or reverse the neurological deficit.

Methods: Milrinone was administered immediately at the onset of neurological dysfunction in five cases, after operation in two and prophylactically before surgical resection in two cases. Initial doses were $0.75 \mathrm{mcg} / \mathrm{kg} / \mathrm{min}$ and were decreased over $2-5$ days.

Results: The administration of Milrinone did not alter intraoperative control of bleeding, BPor HR and no cases of intracerebral hemorrhage were seen. In two cases, intra-operative administration resulted in an immediate improvement in neurological function and in another, a progressive defect stabilized. Postoperative administration was not associated with consistent improvement. Prophylactic administration during MCA vessel sacrifice and tumour resection was associated with our ability to remove difficult lesions without neurological deficit.

Conclusions: Although cerebral tissue injury may occur for a variety of reasons during the surgical removal of tumours under neuroleptic anaesthesia, the prophylactic and immediate administration of Milrinone appears to be safe and may improve patient outcome.

\section{P-105}

Synchrotron-supported analysis of malignant brain tumors: a new diagnostic tool?

E Schultke*, S Hattingh, S Hayat Khan, B Juurlink, R Griebel, (Saskatoon, SK); K Siu, R Lewis, (Melbourne, Australia); C Hall, (Daresbury, $U K$ )

Background: Although glioblastoma multiforme is characterized by an overall poor prognosis, survival can range from less than one month to more than two years from the time of diagnosis. This variance can be observed even between patients with a similar state of disease at the time of diagnosis and in identical treatment protocols. To investigate whether differences at the ultra-structural level could be responsible for the varied outcomes of patients with tumors grouped in the same class by conventional neuropathology, we employed several synchrotron-supported diagnostic techniques.

Methods: Small-angle and wide-angle scattering analysis techniques were used to detect variations in the myelin structure of glioblastoma samples. Infrared microspectroscopy was employed to detect differences in chemical composition.

The long-term goal of this study is to answer the question 
whether differences in structure and composition are correlated to differences in response to therapy and / or to survival time.

This research was supported by the Saskatchewan Synchrotron Institute and the Royal University Hospital Foundation.

\section{P-106}

\section{GFAPand fascin expression in astrocytomas of different grades}

\section{A Peraud*, S Mondal, J Rutka, (Toronto, ON)}

Background: The malignant potential of astroglial tumors consists, apart from tumor growth, in their inherent tendency to invade surrounding brain structures. The regulation of the cytoskeleton is mainly responsible for cell mobility and invasive behaviour. Intermediate filaments such as GFAPand actin fibres are important cytoskeletal components, and fascin is an actin bundling protein. Preliminary results suggest that GFAP and fascin interact, and in the present investigation we show their expression and spatial distribution within glial tumor cells of different grades.

Methods: Paraffin embedded tumor specimens from 46 patients with tumors of different WHO grades were investigated for immunohistochemical expression of GFAPand fascin. The extent of protein expression, the colocalisation with regard to tumor differentiation were investigated.

Results: While the expression of GFAP decreased with increasing grade of malignancy, the amount of fascin positive tumor cells increased and showed highest expression levels in glioblastomas.

The extent of colocalisation of GFAP and fascin is maximal in low grade gliomas and only single cells stain simultaneously positive for GFAP and fascin in malignant gliomas.

Conclusions: Expression of the actin bundling protein fascin increases with tumor progression in gliomas, while GFAP expression shows an inverse expression pattern. Fascin and GFAP were found to be colocalized in single cells, suggesting that they may interact on a protein and/or molecular basis which give rise to further investigations.

\section{P-107}

\section{Glioneuronal tumor with rosetted islands arising in the adult cerebrum}

P Jensen*, J Barron, D Murray, P Bartlett, (St John's, NL);

D Ramsay, (London, ON)

Background: Glioneuronal tumors are uncommon neoplasms that arise in the central nervous system. They may be confused both radiologically and pathologically with other neoplasms such as gangliogliomas, dysembryoplastic neuroepithelial tumors, and other malignant tumors that primarily arise in the brain. We present a case of a glioneuronal tumor arising within the left parietal lobe in a 45year-old male, which radiologically was thought to be a high grade glioma.

Methods: All relevant history and physical examination findings were extracted from the chart. Radiographic examinations were reviewed. Microscopic and immunohistochemical examination of the surgical specimen was conducted both locally and repeated in another referral centre.

Results: CT scanning demonstrated an irregular ring enhancing mass in the left partial lobe with an enhancing nodule on the medial aspect. There was significant associated edema and mass effect. Histology showed the tumor to be composed of independent glial and neuronal components staining appropriately for specific markers. The neuronal component was composed of small monomorphic cells, which arranged in a characteristic rosetted pattern.

Conclusions: This uncommon primary CNS neoplasm can present with features similar to higher grade tumors. Previously reported cases, however, suggest that the tumor usually behaves in a more indolent fashion.

\section{P-108}

Orbital pseudotumor after intra-arterial carboplatin: report of three cases

A Desjardins*, J Salame, A Benko, F Evoy, D Fortin, (Sherbrooke, $Q C)$

Background: Orbital pseudotumor is an inflammatory condition of extraocular muscles. Patients usually experience sudden diplopia associated with orbital pain, conjunctival chemosis, and proptosis. Many etiologies are responsible for this condition.

Methods: We report three patients treated for malignant brain tumors at the Sherbrooke University Hospital with intra-arterial carboplatin enhanced by osmotic blood-brain barrier disruption which developed orbital pseudotumor.

Cases report: Three out of 110 patients treated with this strategy at our institution developed orbital pseudotumor, a few hours to days after the procedure. A 41-year-old man with glioblastoma multiforme, a 67-year-old woman with metastasis of systemic lymphoma, and a 36-year-old woman with brain metastasis of breast cancer. Imaging confirmed the diagnosis in those patients. We observed a good response to systemic steroids in two patients. To prevent relapses, we decided to perform supraselective positioning of the intra-arterial catheter above the ophthalmic artery for infusion of carboplatin. Those strategies were effective in two of the three patients.

Conclusions: We report three cases of orbital pseudotumor complicating treatment with intra-arterial carboplatin for malignant brain tumors. Steroids proved their efficacy as a treatment strategy and supraselective positioning above the ophthalmic artery as a way to prevent relapses.

\section{P-109}

5-fluorouracil increases the efficacy of the herpes simplex virus thymidine kinase/gancyclovir strategy for the treatment of malignant glioma

\section{G Roullin*, E Germain, M Caruso, (Quebec City, QC)}

Background: Gene therapy using the herpes simplex virus thymidine kinase (TK) represents a promising strategy to treat malignant gliomas. TK gene transfer associated with gancyclovir (GCV) can lead to the death of TK-expressing cells, and of neighbouring noninfected cells due to the bystander effect. It has been reported that 5-fluorouracil (5-FU) can increase GCVkilling of TK-expressing cells by decreasing the pool of thymidine, the major competitor of GCV phosphorylation.

Methods: The association of GCV+5-FU was investigated in vitro in four human and three rat glioma cell lines expressing TK30. 
We had previously reported that TK30 is more potent than TK (10to 100-fold) to induce tumour cell killing with GCV.

Results: As calculated by the Chou-Talalay method, at least an additive effect was noted in proliferation experiments for TK30expressing cells, and a potentiation was observed in bystander experiments (mixtures of TK30 and parental cells). Interestingly, a strong synergistic effect was found when parental cells alone were treated with GCV+5-FU. The mechanisms of this anti-tumour effect as well as in vivo studies are presently under investigation.

Conclusions: Thus, the local delivery of GCV+5-FU could contribute to the success of the TK/GCV strategy by efficiently eliminating noninfected tumour cells.

\section{P-110}

\section{Surgical management of an isolated calvarial metastasis: case report and review of the literature}

C DeSilva*, A Kirkwood, D Macdonald, D Ramsay, J Megyesi, (London, $O N$ )

Background: It is not unusual for malignancies such as breast, lung and prostate to metastasize to the brain or calvarium, as well as systemically. However, isolated calvarial metastases are relatively rare: surgical removal of isolated calvarial metastases accounts for less than $0.5 \%$ of cranial tumor operations. Furthermore, isolated calvarial metastases almost always present in patients as a localized swelling without neurological symptoms. We present what appears to be the first report of a patient with an isolated calvarial metastasis that presented with new onset seizures. We review the literature on isolated calvarial metastases and their management.

Clinical Presentation: A 77-year-old female presented with new onset generalized seizures ten years after definitive treatment of localized breast cancer. Her physical examination was within normal limits. CT scanning and MR imaging of her head revealed a destructive left frontal-parietal skull lesion.

Intervention: The skull lesion was resected without opening the dura. Pathological examination of the lesion revealed a moderately differentiated adenocarcinoma. The patient did well postoperatively with no further seizures.

Conclusions: Review of the literature indicates that isolated calvarial metastases occur infrequently and that they rarely present with neurological symptoms. Primary surgical treatment of these lesions can usually relieve the symptoms in symptomatic patients and can also reduce the tumor burden for adjuvant therapy.

\section{P-111}

Angiogenesis in brain tumors: correlation between increasing tumor grade and angiopoietin-2 levels as measured by PCR

C Onochie*, R Hammond, D Gorassini, J Megyesi, (London, ON)

Background: Angiogenesis occurs during solid tumor progression and brain tumors are among the most angiogenic tumors in humans. Angiopoietin-1 (ANG-1) and angiopoietin-2 (ANG-2) are secreted protein ligands for the TIE-2 (tyrosine kinase immunoglobulin and epidermal growth factor homology) receptor expressed on the surface of endothelial cells. ANG-1 promotes vessel integrity by stabilizing interactions between endothelial cells and the surrounding matrix and pericytes. ANG-2 blocks the action of ANG-1 by interfering with the recruitment of pericytes, thereby inducing vessel destabilization. In the presence of vascular endothelial growth factor (VEGF), ANG-2 leads to neovascularization. This study was designed to determine the correlation, if any, between ANG-1 and ANG-2 levels and the pathological grade of brain tumor specimens.

Methods: Human brain tumor specimens were obtained from the Brain Tumor Tissue Bank in London, Ontario. Specimens included oligodendrogliomas, anaplastic oligodendrogliomas and glioblastoma multiforme. The polymerase chain reaction (PCR) was used to quantify ANG-1 and ANG-2 mRNAexpression in the specimens.

Results: Statistical analysis revealed a strong correlation between increasing tumor grade and the level of ANG-2 expression. There was no correlation between tumor type and the level of ANG-1 expression.

Conclusions: In certain types of brain tumors the expression of ANG-2 correlates with tumor grade and angiogenic capacity.

\section{P-112}

Aspiration of a mesencephalic cyst with resolution of unilateral parkinsonian-type tremor: a case report

RT Grondin*, BM Wheatley, TE Roberts, JDS McKean,

(Edmonton, $A B$ )

Background: There are only two case reports of a unilateral parkinsonian tremor secondary to a mesencephalic cyst. This is the first description of the successful treatment of this symptom complex.

Case report: We report the case of a 59-year-old man with a unilateral parkinsonian syndrome secondary to a multicystic mesencephalic lesion. The patient presented with a five year history of right upper extremity resting tremor and vertical diplopia. Magnetic resonance imaging (MRI) demonstrated a nonenhancing, multiloculated cystic lesion in the left midbrain with extension into the pons and thalamus. Stereotactic CTguided aspiration of the cyst revealed serous fluid. No atypical cells were identified on cytology. Following the procedure, the patient had a significant improvement in his tremor. His UPDRS (united Parkinson disease rating scale) score improved from 12 to 10 . Six months later, his tremor had recurred. A CT scan demonstrated an increase in the size of the cyst. An attempted third ventriculoscopic cyst fenestration resulted in no significant improvement in his symptoms. An MRI guided frameless stereotactic insertion of an Ommaya reservoir resulted in complete resolution of his tremor.

Conclusions: Cyst aspiration is a treatment option for patients with mesencephalic cysts causing parkinsonian symptoms.

\section{P-113}

Leptomeningeal gliomatosis: diagnostic and management challenges

M Bangash*, M Dionne, CE Maxner, RJB Macaulay, IG Fleetwood, (Halifax, NS); A Engelbrecht, (St John's, NL)

Background: Leptomeningeal gliomatosis (LG) is a rare neoplasm, seldom encountered in clinical practice and often demonstrating a fulminating fatal clinical course.

Methods: We report one patient with LG and review the literature, focusing on diagnostic and management challenges.

Results: This young healthy man presented with nonspecific 
symptoms and signs of meningeal irritation with intermittently elevated intracranial pressure. While MRI demonstrated diffuse leptomeningeal enhancement, dozens of other investigations were negative, including repeated CSF studies and a brain and leptomeningeal biopsy. His case was reviewed at several institutions without diagnosis. The patient eventually succumbed secondary to brainstem dysfunction and aspiration. Autopsy findings diagnosed LG.

Conclusions: LG represents a diagnostic dilemma and while confirmation of diagnosis does not yield any definitive treatment options, there are implications for patient management.

\section{P-114}

Stereotaxic radiosurgery in patients with brain metastasis: University of Montreal Health Center experience

E Sauvageau*, R Moumdjian, JP Bahary, MA Fortin, (Montreal, $Q C)$

Background: Brain metastasis commonly occur in patients with cancer. Steroids, whole-brain radiation therapy and surgery are usual therapeutic modalities used in their treatment. Over the last years, stereotactic radiosurgery has gained popularity as an adjunct in the treatment of those lesions. Patients treated for brain metastasis in our institution using linear accelerator stereotactic radiosurgery have been reviewed.

Methods: 63 selected patients with 89 brain metastasis underwent stereotactic radiosurgery between 1996 and 2002 in our institution.

Results: The mean age of patients was 51 (range 23-74 years). There were 27 men and 36 women. The primary tumor location was lung (41 patients), breast (7 patients), kidney (3), melanoma (2) and others (10 patients). $68 \%$ of patients harboured one lesion, $22 \%$ two lesions and $10 \%$ had three lesions. LINAC based stereotactic radiosurgery was used. Single session was done even if multiple lesions were treated. $80 \%$ of the lesion was treated by a unique isocenter. The mean radiation dose administered was 17,2 Gy. The majority of patients underwent WBRT and surgery for another lesion as well. Four patients received only radiosurgery. 54 out of 63 patients were available for review. Progression occurred in $22 \%$ after treatment. Radiation-induced necrosis has been suspected in nine patients. Median survival time was 10.7 months postdiagnosis.

Conclusions: Stereotactic radiosurgery is an interesting alternative treatment of brain metastasis. Decrease and stabilization in tumor size was achieved in $78 \%$ of our patients. Radiationinduced necrosis was higher than reported in the literature.

\section{P-115}

\section{Third ventricle high-grade astrocytoma}

\section{Crevier*, J Provias, N Murty, (Hamilton, ON)}

Background: Although malignant astrocytomas can theoretically be found anywhere in the CNS, only a few cases of primary intraventricular high-grade astrocytomas have been reported in the literature.

Methods: This report describes one case and reviews the relevant literature.

Results: The patient was a 71-year-old male who presented with a one month history of progressive difficulty walking, short-term memory problems, diffuse headaches, and vomiting. Computerized tomography and magnetic resonance showed an irregularly enhancing lesion encasing and involving the third ventricle, causing moderate obstructive hydrocephalus. A Stealth-CT-guided endoscopic biopsy and septostomy were performed. Permanent sections demonstrated an astrocytic neoplasm with significant pleomorphism and several atypical tumour cells. The final diagnosis was high grade astrocytoma/probable glioblastoma multiforme.

Conclusions: The origin of these lesions is still unclear, but a few structures have been suggested in the past: septum pellucidum, fornix, periventricular parenchyma. The optimal treatment remains controversial and will be discussed. Although of rare occurrence, malignant astrocytoma should be included in the differential diagnosis of a third ventricle enhancing lesion.

\section{P-116}

A modification to an animal model for osmotic blood-brain barrier disruption eliminating hemodynamic effects produced by anaesthetic agents

$R$ Adams*, A Desjardins, D Fortin, (Sherbrooke, QC); A Gallez, (Montreal, $Q C$ )

Objective: We propose a modification to a blood-brain barrier disruption (BBBD) model that allows the use ketamine/xylazine as anesthetic, decreasing the complexity and the cost of the model, while maintaining efficiency.

Methods: Forty-seven Long Evans rats were anesthetized by intraperitoneal injection of ketamine/xylazine. Osmotic BBBD was performed by administering $25 \%$ mannitol into the internal carotid artery in a retrograde fashion from the external carotid. The infusion rate of mannitol as well as the duration were adjusted in a stepwise fashion to identify optimal parameters. As a supplementary step, a vascular clip was applied to the common carotid artery (CCA) prior to the infusion of mannitol, isolating our model system from the hemodynamic effects of ketamine/xylazine. Evans blue was used to control for BBBD intensity.

Results: Using this model at an initial infusion rate of $0.15 \mathrm{ml} / \mathrm{sec}$ and decreasing the infusion rate in a stepwise fashion, $0.08 \mathrm{ml} / \mathrm{sec}$ was found to produce optimal BBBD. At this rate, we obtained six grade II and 1 grade IV staining.

Conclusions: The application of a clip to the CCA prior to mannitol infusion allowed us to isolate the cerebral circulation from the hemodynamic effects of ketamine/xylazine producing consistent BBBD in our model.

\section{P-117}

Mass spectrometric analysis of aberrant signaling complexes mediated through wild type and mutant epidermal growth factor receptors in human glioblastoma multiforme

\section{J Micallef*, M Moran, A Guha, (Toronto, ON); J Caldwell, (Charlottesville, VA)}

Background: Glioblastoma multiforme (GBM) are the most malignant and common form of astrocytomas in adults. They are frequently characterized by expression of several mutant forms of the epidermal growth factor receptor (EGFR), the most common being EGFRvIII and EGFR-C958. These mutant receptors confer a growth advantage to GBMs. We are elucidating the biochemical 
pathways utilized by these receptors using mass spectral analysis.

Methods: Established human GBM cell lines expressing EGFRvIII and EGFR-C958, GBM explants and operative specimens characterized for mutant EGFRs, are being analyzed by mass spectrometry to determine 1) the total protein-profile and totalphosphotyrosine profile of tumors harboring EGFRvIII and EGFRC958; 2) the intracellular signaling molecules that associate with EGFRvIII and EGFR-C958; 3) the phosphorylated residues of EGFRvIII and EGFR-C958

Results: Several proteins have been found to be differentially upregulated in human xenograft GBM explants expressing EGFRvIII with further experiments ongoing to decipher their functional relevance. We are also analyzing GBM explants to determine the differential total phosphotyrosine profiles and to identify signaling proteins that preferentially interact with mutant EGFRs.

Conclusions: An increased understanding of the signaling pathways utilized by mutant EGFRs prevalent in human gliomas, may help in identification of novel therapeutic targets in these currently terminal cancers.

\section{P-118}

\section{MRI correlates of molecular signatures in oligodendrogliomas}

J Megyesi*, E Kachur, D Lee, M Zlatescu, (London, ON); R Betensky, Y Okada, H Sasaki, D Louis, (Boston, Massachusetts); $P$ Forsyth, G Cairncross, (Calgary, Alberta)

Background: Molecular genetic subsets of oligodendroglioma behave in biologically distinct ways:- their locations within the brain, rates of growth, and responses to contemporary cancer therapies.

Methods: In this study, we examined the relationship between molecular genetic alterations and imaging features in newly diagnosed oligodendrogliomas. We inquired whether coincident allelic loss of chromosomal arms $1 \mathrm{p}$ and $19 \mathrm{q}$, an early molecular event in the genesis of a subset of oligodendrogliomas that evolve more slowly and are chemosensitive, was associated with specific characteristics that could be detected by clinical magnetic resonance imaging (MRI).

Results: In a series of 40 cases, we observed that $1 p$ and $19 q$ status was closely associated with two imaging parameters:sharpness of the tumor border on T1-weighted images and the homogeneity of the tumor signal on $\mathrm{T} 1$ and $\mathrm{T} 2$ images. Both an indistinct tumor border on $\mathrm{T} 1(\mathrm{p}=0.005)$ and a mixed intensity signal on $\mathrm{T} 1 / \mathrm{T} 2(\mathrm{p}=0.054)$ were significantly associated with allelic loss of $1 \mathrm{p}$ and $19 \mathrm{q}$. The presence of susceptibility effect was also significantly associated with $1 \mathrm{p}$ and $19 \mathrm{q}$ loss $(\mathrm{p}=0.008)$.

Conclusions: These findings raise the possibility that signature molecular alterations associated with particular types of brain tumors may confer upon the tumor certain physical or biochemical characteristics that can be imaged.

\section{NEURO-OPHTHALMOLOGY}

\section{P-119}

Visual recovery with administration of high-dose vitamin supplements in a patient with Leber's hereditary optic neuropathy

T Lad*, A Patel, (Saskatoon, SK)

Background: Leber's hereditary optic neuropathy is an inherited mitochondrial disease characterized by bilateral, subacute, painless vision loss. Affected individuals are typically young males, between the ages of 15 and 35 . Visual outcome is poor and no specific therapies have shown proven benefit. We report a case of visual recovery in a patient with Leber's hereditary optic neuropathy (mutation 11778) with the administration of high-dose multiple vitamins.

Methods: Observational case report. A 52-year-old female presented with a two month history of gradually progressive, bilateral vision loss. Diagnosis of Leber's hereditary optic neuropathy was made via genetic analysis. Visual acuity, at time of diagnosis, was 20/200 OD, and 5/300 OS. High-dose multivitamin supplements were initiated.

Results: Eight months after initiation of vitamin supplementation almost complete visual recovery was achieved with acuities of 20/25-2 OD and 20/25-1 OS. Visual fields as measured by Goldmann perimetry also showed significant improvement.

Conclusions: High-dose multiple vitamin therapy aided visual recovery in a patient with genetically confirmed Leber's hereditary optic neuropathy.

P-120

Spasmus nutans and congenital ocularmotor apraxia in a patient with cerebellar vermian hypoplasia

JS Kim*, SH Park, KW Lee, (Seoul, Korea)

Background: Spasmus nutans refers to a syndrome of nystagmus, head nodding, and abnormal head position. Congenital ocular motor apraxia (OMA) is characterized by impaired voluntary saccades and abnormal head thrusts to induce a fixation. Both disorders are rare clinical entities and sequential development in a patient with cerebellar vermian hypoplasia has not described.

Case report: A seven-year-old boy, who had a history of spasmus nutans during infancy, presented with typical findings of congenital OMA. He had impaired voluntary saccades and smooth pursuit in the horizontal plane. He showed thrusting movement of the head during attempted gaze shift. He had a history of developmental delay. Brain MRI demonstrated cerebellar vermian hypoplasia, especially in the inferior portion.

Conclusion: Spasmus nutans and congenital OMAshare clinical characteristics and may develop in a patient with cerebellar vermian hypoplasia. Careful evaluation of the cerebellar vermis is needed in patients with these disorders. 
P-121

\section{Three neuro-ophthalmologic cases with giant cell arteritis}

Zahra Hallaji*, (Tehran, Tehran)

Giant cell (temporal or cranial) arteritis (GCA) is an inflammatory vasculopathy of the elderly that affects medium to large-sized arteries. GCA may present with numerous systemic and ocular manifestations. GCA usually causes visual loss due to anterior ischemic optic neuropathy (AION). All patients older than age 50 years with AION should be suspected of having GCA. Although the usual cause of visual loss in GCA is AION or central retinal artery occlusion, posterior ischemic optic neuropathy (PION), choroidal or rarely occipital lobe ischemia may also occur. The differential diagnosis for ocular conditions (acute visual loss, unexplained diplopia, retinal or choroidal ischemia, or transient visual loss) should include GCA. Three cases that are being presented are pseudo foster kennedy syndrome and bilateral AION and VI nerve palsy in a diabetic patient that documented with positive temporal artery biopsy.

\section{NEURO-IMAGING}

\section{P-122}

\section{Ahand rule fordetermining the age of intracranial blood on MRI}

\section{Spivak*, V Chow, (Saskatoon, SK)}

Background: Intracranial hemorrhage is a devastating clinical problem. The timings of these hemorrhages are important for clinical decision-making. These hematomas evolve with time and have been divided into five stages: hyperacute ( $<1$ day), acute (1-3 days), early subacute ( $>3$ days), late subacute ( $>7$ days) and chronic (>14-28 days). Unfortunately, their signal patterns on T1 and T2 weighted images (WI) are difficult to remember. We propose a simple hand rule memorization tool to determine the stage of a hemorrhage on MRI.

Methods: A departmental teaching aid.

Results: Each finger represents a different stage of blood. The little, ring, long, index fingers and thumb represent hyperacute, acute, early subacute, late subacute and chronic blood, respectively. Early and late subacute blood is bright on T1WI. This is represented by the Roman numeral one by extending the long and index fingers. Hyperacute and late subacute blood is bright on T2WI. This is represented by a Roman numeral two by extending the little and index finger. Flexed fingers represent dark (hypointense) blood on T1WI and T2WI.

Conclusions: This memory aid allows the determination of the intensity of blood in different stages of evolution on T1WI and T2WI.

\section{P-123}

\section{Cranial nerve imaging; I-XII}

Mehran Midia*, Robert Vandorpe, (Halifax, NS); Ramin Midia, (Springfield, IL); Zinat Miabi, (Tabriz, Iran); Suresh Mukherji, (Ann Arbor, MI)

Background: Today it is possible to image all cranial nerves utilizing 1.5 Tesla MR magnets, although CT still holds its value in evaluating the orbit and the temporal bone. The following article will review the cranial nerve anatomy relevant to imaging and discuss the common disease entities that can cause cranial nerve dysfunction.

Methods: To better tailor the imaging and understand the different pathologies understanding the cranial nerve anatomy is crucial. Here each cranial nerve has been divided and evaluated into following segments: intra-axial, cisternal, transitional (e.g. cavernous, Dorello's canal, Meckel's cave), intra-osseous and extracranial.

Result: Being familiar with normal and abnormal imaging characteristics of cranial nerve is essential. These include direct (caliber change, disruption, signal or attenuation change, calcification, pathologic enhancement, and mass) and indirect imaging findings (e.g. obliteration of fat planes, disruption of osseous channels or foramina, muscle atrophy, and asymmetric positioning of the tissues).

Conclusions: The aim is to provide information that will help evaluating the cranial nerves. This knowledge would be helpful for physicians dealing with diagnosing and treating patients suffering from cranial nerve dysfunction.

\section{P-124}

Use of CT, CT angiography and CT perfusion to triage patients with hyperacute ischemic stroke: our initial experience

$M$ Goyal $^{*}$, MJ Hogan, M Kingstone, C Lum, W Miller, T Nguyen, M Sharma, P Stys, (Ottawa, ON)

Background: The outcome of hyperacute stroke can be altered by timely intervention with intravenous/intra-arterial thrombolytic therapy. Knowledge of the level of occlusion (ICA, M1 segment of MCA vs. distal circulation) and an assessment of degree of irreversibly damaged brain vs. salvageable tissue helps in decisionmaking. We present our initial experience with CT angiography (CTA) and CT perfusion (CTP) in patients with hyperacute stroke.

Methods: Over the last seven months, 16 patients presenting with stroke within the first six hours underwent a CTA and CTP study. CTA data were analyzed using thick section multiplanar reformat. CBV, CBF and MTT maps were generated off-line. Depending on patient condition, time from onset of stroke, co-morbidities and CT study, a decision for further treatment was taken.

Results: Good quality CTA and CTP studies were obtained in 15 of the 16 patients. CTA allowed evaluation of large vessel occlusion/narrowing. In six patients who underwent conventional angiography following the CT, the findings on angiography matched those on CTA. In four patients who underwent successful recanalization by intervention through the arterial route, the areas that showed normal CBV but low CBF and high MTT in the initial CT perfusion survived on the follow-up CT scan but areas that had low $\mathrm{CBV}$ resulted in infarction.

Conclusion: CT(with CTA and CTP) has the potential to provide information on large vessel occlusion and tissue viability in patients with hyperacute stroke and could help further refine management. 


\section{P-125}

\section{Spinal sarcoidosis: an occult diagnosis}

R Jassal*, S Hussain, W Johnston, (Edmonton, $A B$ )

Background: Sarcoidosis is an idiopathic, multisystem granulomatous disease with rare CNS (including spinal cord) involvement (5\% of cases). Radiographically, neurosarcoidosis can mimic other CNS lesions. In the absence of lung involvement, sarcoidosis might not be considered in the differential diagnosis of myelopathy.

Methods: Case report/literature review

Results: A previously healthy 67-year-old woman presented with a one-year history of progressive upper extremity weakness, fasciculations and sensory changes accompanied by clinical myelopathy. EMG studies revealed upper extremity chronic denervation and reinnervation. Cervical MRI showed spondylosis with markedly increased spinal T2 signal change. Investigations, including a serum ACE level and chest X-ray, were normal. Postoperatively, clinical and radiographic progression, interpreted as consistent with astrocytoma, occurred. Cerebrospinal fluid analysis revealed a lymphocytic pleocytosis (polyclonal) and a mildly elevated protein. Gallium scan revealed increased uptake in hilar, mediastinal and abdominal lymph nodes; CT scan of the chest and abdomen revealed adenopathy. Lymph node biopsies revealed granulomas consistent with sarcoidosis. Treatment with steroids was initiated. Follow-up MRI showed decreased gadolinium enhancement. After initial clinical improvement, the patient deteriorated.

Conclusions: Neurosarcoidosis can be a difficult diagnosis to make, and should be considered in the differential of spinal cord lesions.

\section{Neuromuscular Disease}

\section{P-126}

\section{Lupus and polymyositis presenting as an overlap syndrome}

JA Pettersen*, RB Bell, R Ranawaya, AKW Brownell, C White, (Calgary, $A B)$

Background: Lupus and polymyositis co-occur as an overlap syndrome in 5\% of lupus patients. Criteria must be fulfilled for both lupus and polymyositis to make this diagnosis.

\section{Methods: Case report}

Results: A 32-year-old woman presented with a five-month history of progressive proximal muscle weakness, myalgias, fevers, generalized edema, and fatigue. On examination, she was febrile (39.9 C) with effusions of her wrists and PIP joints, profound bilateral proximal muscle weakness. Investigations demonstrated a normocytic anemia, hypoalbuminemia, positive ANA (1:640), antiDNA and anti-Jo-1 antibodies, decreased complement levels, elevated LDH (8 times normal) and CK (16300 U/L; normal: 0-170 $\mathrm{U} / \mathrm{L})$. Urinanalysis revealed myoglobinuria, proteinuria and microscopic hematuria. Renal biopsy was consistent with lupus nephritis while EMG and muscle biopsy were consistent with polymyositis. As she did not improve on prednisone $50 \mathrm{mg} /$ day over one month, cyclophosphamide $1200 \mathrm{mg}$ iv was administered. However, she was re-admitted 10 days later with progressive weakness. Intravenous gamma-globulin $2 \mathrm{mg} / \mathrm{kg}$ was given over five days. Within one month, her strength improved with a concomitant decrease in CK (3000 U/L).

Conclusions: Although rare, lupus-polymyositis overlap does occur, and needs to be recognized since more aggressive treatment may be required than for either of the entities when they occur separately.

P-127

Co-incident anticholinergic receptor antibody and anti-asialo GM1 antibody in a young male

$C$ Toth*, C White, (Calgary, AB)

Background: Co-existence of myasthenia gravis with an immunologically mediated neuropathy syndrome is rare.

Methods: We present a young male patient with a history of generalized muscle weakness and fatigue.

Results: Electrophysiological evidence of both a motor neuron syndrome and a neuromuscular junction abnormality was found. Muscle biopsy was supportive of the existence of a motor neuropathy. Laboratory evidence for both acetylcholine receptor antibodies and anti-asialo GM1 antibodies, both of high levels, were found. The patient's fatigue and weakness showed a subjective response to pyridostigmine and prednisone, with objective improvement with intravenous immunoglobulin.

Conclusions: The combination of electrophysiologically and laboratory proven myasthenia gravis and immune-mediated lower motor neuron syndrome is a rare clinical entity.

\section{P-128}

\section{Mononeuritis multiplex in association with livedoid vasculitis}

C Toth*, D Zochodne, (Calgary, AB)

Background: Livedoid vasculitis is a chronic dermatological disorder affecting the feet and lower legs with petechiae and recurrent, bizarrely shaped ulcers which heal to form hyperpigmented areas and atrophie blanche. This condition is more correctly termed a vasculopathy, rather than a vasculitis, and is often associated with an underlying hypercoagulable disorder. Involvement of the nervous system with livedoid vasculitis is distinctly unusual.

Methods: We report the case of a young female with livedoid vasculitis and mononeuritis multiplex.

Results: Skin biopsy was consistent with livedoid vasculitis. Electrophysiological data and nerve biopsy were consistent with multifocal axonal neuropathy. No evidence of a hypercoagulable disorder could be found.

Conclusions: Livedoid vasculitis is a rare association of mononeuritis multiplex. We report the only patient in the literature with livedoid vasculitis and clinical, electrophysiological, and pathological evidence of a concurrent neuropathy. We propose that peripheral nervous system involvement was due to multifocal areas of ischemia due to suspected fibrin and thrombin deposition within both the wall and lumen of affected vessels, including vasa nervosum. 
P-129

Motogenic factors modify the migration of human myoblasts in vivo

P Mills*, J-F Lafreniëre, JP Tremblay, E El Fahime, (Quebec City, $Q C)$

Background: A potential treatment of Duchenne muscular dystrophy (DMD) is the transplantation of myoblasts. A major problem is the poor migration of transplanted myoblasts. We have previously shown that the metalloproteinases (MMPs) and urokinase plasminogen system are implicated in the in vivo migration of mouse myoblasts. It has been demonstrate that motogenic factors could upregulate these proteolytic systems. We therefore decided to test whether b-FGF and IGF-1 could improve the in vivo migration of human myoblasts.

Methods: To evaluate the effect of the motogenic factors on the MMPs and the urokinase plasminogen system, we used western blot and zymography. The in vivo migration was quantified with the micro-tube technique described in El Fahime E. et al: Exp Cell Res, 2000.

Results: In vitro, our results show that the presence of b-FGF or IGF-1 enhance the expression of some MMPs and some components of urokinase plasminogen system. In vivo, our results show that bFGF and IGF-1 co-injection improves the migration of transplanted myoblasts. The migration distance of human myoblasts was about 200 um without treatment, 900-950 um in the presence of IGF-1 and roughly $500 \mathrm{um}$ in the presence of b-FGF.

Conclusion: These results indicate that the co-injection of IGF-1 is to be more effective and could help to reduce the number of injection sites in myoblast transplantation for the treatment of DMD patients.

\section{P-130}

\section{A tolerance induction protocol for myoblast transplantation}

\section{Stephan*, J-P Tremblay, (Quebec, QC)}

Background: We work on cell therapy based treatment for Duchenne muscular dystrophy (DMD) disease. However, cell therapy requires adequate immunosuppressors and/or tolerance induction to prevent allograft rejection. Transplantation tolerance has been associated with stable donor chimerism following bone marrow cell transplantation. Chimerism induction has been established with a drug protocol method (cyclophosphamide and busulphan). We have tested whether chimerism induction with that protocol permitted to develop immunological tolerance for myoblast transplantation.

Methods: Mdx/mdx mice, DMD animal experimental model, were intravenously injected with $60.106 \mathrm{Balb} / \mathrm{c}$ spleen cells (day 0), intraperitoneally injected with cyclophosphamide (200 mg.kg-1) and busulfan (25 mg.kg-1) (day 2), intravenously injected with 15.106 Balb/c bone marrow cells depleted of T-cells (BMT) (day3), and grafted in the tibialis anterior with $1.106 \mathrm{Balb} / \mathrm{c}$ primary myoblasts (day 30). After 140 days, chimerism level was determined by FACS, and graft acceptation was determined by the presence of dystrophin positive fibers by immunohistochemical staining.

Results: At three months after BMT, in treated mice we observed around $30 \%$ chimerism and several hundred dystrophin positive fibers due to the fusion of donor myoblasts with the host muscle fibers.

Conclusions: Specific tolerance has been obtained. We will now investigate whether it is possible to do successive transplantations of myoblasts from the same donor without repeating the tolerigenic treatment and also investigate immunological tolerance specificity by transplanting myoblasts obtained from a third mouse strain to the tolerigenic host.

\section{P-131}

Isolated bilateral phrenic neuropathies - a rare presentation of neuralgic amyotrophy

ZA Siddiqi*, SN Ahmed, (Edmonton, AB)

Background: Phrenic nerve involvement is a known complication of brachial neuralgic amyotrophy (NA) though isolated involvement of both phrenic nerves in NA is exceedingly rare.

Methods: A 33-year-old obese previously healthy female woke up with severe left shoulder pain followed by worsening shortness of breath. Two weeks later she developed pain in the right shoulder with further worsening of dyspnea and inability to lie flat. Clinical findings included tachypnea, reduced breath sounds at bases and paradoxical abdominal breathing when supine. She had a normal neurological examination. Workup showed normal hematological studies with reduced vital capacity and hypoxia. Electromyography revealed severe denervation of the diaphragm with no recordable motor unit potentials. No electrophysiological abnormality was detected in the right upper extremity and cervical paraspinal muscles. A subsequent fluoroscopic examination confirmed bilateral diaphragmatic paralysis.

Results: Based on the clinical diagnosis of NA the patient was given a course of IVIG. At one month follow-up she reports improvement in dyspnea though still requires BiPAPwhen sleeping.

Conclusions: Isolated bilateral phrenic neuropathy leading to severe respiratory insufficiency is a rare presentation of NA. Early recognition and aggressive management of this entity may improve outcome and prevent prolonged dependency on ventilation devices.

\section{NEUROPHYSIOLOGY}

\section{P-132}

Three cases of cauda equina syndrome due to bupivacaine neurotoxicity

Sophie Gagnon*, (Gatineau, QC)

Background: A medline research from 1966 to 2002 (keywords: cauda equina syndromes, anesthesia epidural) identified three cauda equina syndrome caused by a traumatic anesthesia. No pathophysiologic hypothesis was suggested.

Method: In 2002, three patients came to a electromyography clinic with a complete or partial cauda equina syndrome following an epidural or rachianesthesia with bupivacaine. Each patient had an electromyogram and a lumbosacral spine magnetic resonance imaging (MRI) to rule out any structural lesion (hematoma, abscess, protruding disc). Electromyographic or clinical follow-up was done for all patients.

Results: All MRI were normal. All electromyograms showed 
sensory and/or motor axonal disease. Slow clinical proximal to distal improvement correlated well with electromyographic proximal to distal decreasing of pathologic spontaneous activity and increasing of re-innervation activity.

Conclusion: We believe bupivacaine epidural and rachianesthesia can caused a cauda equina syndrome: neurotoxic wallerian degerescence is followed by slow proximal to distal reinnervation. Though infrequent, this could usefully be added to our differential diagnosis.

\section{Pediatric Neurosurgery}

\section{P-133}

\section{Favorable response of a desmoplastic infantile astrocytoma to chemotherapy}

D McNeely*, (Halifax, NS); V Mehta, (Edmonton, AB)

Background: Desmoplastic infantile astrocytoma (DIA) are rare tumors that tend to affect children under the age of eighteen months. These tumors are usually supratentorial with a cystic component and treated with radical excision with good results. Occasionally other treatments including radiotherapy and chemotherapy have been employed.

Methods: We present a case of a four-month-old female who presented with a two-week history of complex partial seizures. A left homonymous hemianopsia was documented at presentation. MRI imaging demonstrated an enhancing tumor involving the right medial temporal lobe, hypothalamus, optic chiasm and encasing the internal carotid artery.

Results: This tumor was not felt to be completely resectable. We performed an open biopsy, followed by a VP shunt. Pathological confirmation of a DIA was made, with areas expressing a MIB-1 proliferative index of up to $9 \%$. The child was placed on a chemotherapy regimen of vincristine and carboplatin for one year with good clinical and radiographic response.

Conclusions: When complete surgical resection of a desmoplastic infantile astrocytoma is not feasible, alternative strategies are required. The rarity of DIAs, have precluded the development of any uniform adjuvant treatment protocols. This represents the first case reported using this type of a chemotherapy regimen. Carboplatin and vincristine together appear to represent a safe and effective chemotherapeutic regimen for DIAs.

\section{P-134}

\section{Unusual presentation of spinal cord injury without radiographic abnormality with disproportionate weakness of lower extremities - a case report}

F Pirouzmand, S Ebenezer*, (Saskatoon, SK)

Background: Spinal cord injury without radiographic abnormality (SCIWORA) is a disease of pediatric population. The presentation of these injuries is dependent on the location and severity of cervical cord injury. The usual manifestation is disproportionate weakness of upper versus lower extremities. We report a toddler with an unusual presentation of paraplegia and good upper extremities strength after a SCIWORA injury. The initial radiographic appearance of holocord swelling in the cervical spine with subsequent rapid resolution of magnetic resonance imaging
(MRI) findings and more gradual neurological recovery is described. Methods: We present a 25-month-old female, who landed on her head while somersaulting off a sofa. She presented after one day with paralysis of the lower extremities, and close to normal strength in the upper extremities. After appropriate radiological imaging, a diagnosis of SCIWORA was made. This patient was followed radiologically with MRI three weeks after the accident and clinically up to now (2.5 months).

Results: An initial MRI revealed hyperintense signal in T2, and expansion of the whole cervical cord. The majority of cord signal and volume changes were centered at $\mathrm{C} 2$ level. There was no obvious evidence of significant ligamentous disruption. A follow-up MRI after three weeks confirmed almost complete resolution of signal changes and edema of the cord. The patient initial presentation was extremely unusual with disproportionate paralysis of lower extremities and sparing of upper extremities despite the high location of the center of cord injury. Further clinical follow-up showed significant improvement of function in the lower extremities (early follow-up in 2.5 months).

Conclusions: Patients with cervical spinal cord injuries traditionally present with weakness or paralysis of the hands and arms with relative preservation of lower extremity strength. The above case describes a high cervical spinal cord injury that resulted in paralysis of the lower extremities, with preservation of upper extremity strength. This finding cannot be explained by the currently accepted pathophysiology of cervical spinal cord injury. The rapid recovery of diffuse cord changes in MRI and subsequent recovery of leg symptoms (although incomplete) demonstrate lack of strong predictive value for both initial MRI and neurological findings.

\section{P-135}

Pott's puffy tumor in a three-year-old child

V Mehta*, M Gupta, R Bhargava, H El-Hakim, (Edmonton, AB)

Background: Sir Percival Pott, 200 hundred years ago described a subperiosteal abscess of the frontal bone appearing as a localized swelling of the forehead. This presentation has become known as "Pott's puffy tumor" and represents an infectious complication usually associated with frontal sinusitis.

Methods: A three-year-old otherwise healthy child presented with a two-week history of a flu-like illness. After this period the family noticed an egg-sized swelling over his forehead. This soft tissue swelling was neither tender nor erythematous. The child was placed on clindamycin and a neurosurgical consult was obtained.

Results: This child was afebrile with a normal white blood cell count. A skull x-ray, CTscan and MRI were all abnormal. There was demonstration of a frontal sinus, subgaleal soft tissue, erosion of the skull combined with an epidural abscess. The patient was brought to the operating room and the osteomyelitic bone was debrided and the epidural abscess removed.

Conclusions: Pott's puffy tumor's has rarely been reported in the literature since the introduction of antibiotics and most cases involve children in late adolescence. Although traditional teaching has suggested that a frontal sinus is usually not formed by the age of three, this case demonstrates that one should not discard the diagnosis of Pott's puffy tumor in this age group. 


\section{P-136}

The effect of craniotomy on cranio-orbital growth asymmetry in children

\section{A Wu*, F Pirouzmand, (Saskatoon, SK)}

Background: Most of the skull growth occurs in childhood. Craniotomy can potentially influence this growth. Computed tomography (CT) has been shown to be reliable in quantitatively measuring skull growth. This study was conducted to apply these CT measurements to evaluate the effect of craniotomy on the skull growth in the pediatric population.

Methods: This is a retrospective cohort study of children with normal craniofacial anatomy, who underwent craniotomy in a single neurosurgical hospital from 1980 to 2002. Pre-operative and subsequent follow-up CTand/or MRI imaging of these patients were converted to digital format and analyzed using the Canvas $7.0^{\mathrm{TM}}$ software. The following measurements were obtained from each image: the coronal distance to midline both at the frontal ventricular tips and maximum biparietal diameter levels, the anterior lateral orbital distance to midline, lateral orbital wall length, and lateral orbital wall angle. Right and left sided radiographic measurements were compared to assess asymmetry, and the degree of asymmetry was measured over time.

Results: On completion, we expect to have a final patient population of about 30 patients. We expect to find a small to moderate degree of asymmetry with lesser growth on the side of the craniotomy. The effect of different independent variables on this growth modification is currently under investigation (will be presented in final paper).

Conclusions: The effect of their craniotomy on long-term craniofacial growth may have cosmetic implications. This effect has further implications for methods of rigid skull fixation. It also can help to understand the effect of cranial sutures or other independent variables on the normal mechanism of skull growth.

\section{P-137}

\section{Visual outcome following resection of craniopharyngiomas in children}

\section{$M$ Kelly*, D Coupal, V Sadanand, AD Patel, R Griebel, (Saskatoon, $S K$ )}

Background: Craniopharyngioma is a benign tumor thought to arise from remnants of Rathke's pouch and causes of impaired visual function in children. This study reviews the impact of this tumor and subsequent surgery on vision.

Methods: Seven patients under the age of ten underwent surgical excision of craniopharyngiomas. All patients had pre- and postoperative ophthalmalogic examinations of visual acuity, visual fields, fundoscopy and ocular motility. Patient numbers were too small to allow statistical analysis.

Results: Seven patients underwent 11 procedures. Five patients had gross total resection and two patients had subtotal resection at the initial surgery. Recurrence was seen in two patients with gross total resection and two patients with subtotal resection. One patient who was completely blind pre-operatively had no change following surgery. Two patients had no pre-operative visual compromise. One patient with visual field deterioration with recurrence improved following repeat resection. Four patients had improvement in visual acuity and fields with surgery. Visual function was most improved in two patients who had no pre-operative evidence of optic nerve atrophy.

Conclusions: Visual acuity and visual field deficits are likely to improve with operative treatment of craniopharyngioma. Absence of optic nerve atrophy in patients with craniopharyngioma is an important prognostic indicator of visual recovery following surgical resection.

\section{P-138}

Split cord malformation associated with a lipoma, dermal sinus tract and teratoma: report of two cases

V Sadanand*, M Kelly, R Griebel, (Saskatoon, SK)

Background: Split cord malformations (SCM) are rare congenital spinal dysraphic states that are frequently associated with other spinal anomalies. True spinal teratomas are even rarer congenital abnormalities. The combination of SCM and a teratoma is extremely uncommon with only five previously reported cases. We report two cases which together with other reported cases suggests a common dysembryogenic origin in a dysplastic endomesenchymal tract.

Methods: The first patient presented at birth with an SCM type 1, myelomeningocele, dermal sinus tract and a mature teratoma. The second patient was a four-day-old female with an SCM type 1, lipomyelomeningocele, dermal sinus tract and an immature teratoma.

Results: The patients underwent magnetic resonance and computed tomographic imaging of the spine. Surgical excision of the bony septum was performed in one case and both cases had good closure of the MMC. Pathological material in both cases confirmed the diagnosis and will be presented.

Conclusions: We present two cases of an extremely rare combination of a split cord malformation and teratoma. While teratomas have arguably been thought of as a neoplastic entity, our cases and the few reports in the literature suggests that they may have an origin in the persistent endomesenchymal tract that gives rise to the SCM.

\section{P-139}

\section{Thalamic tumors in children}

$R$ Yahya*, J Drake, (Toronto, ON)

Background: Thalamic tumors are generally low grade tumors that occur more commonly in children than in adults. They represent $1-5 \%$ of brain tumors and most series include both adult and pediatric patients. This report summarizes our experience with thalamic tumors in children under 18 years of age over the last 15 years.

Methods: We retrospectively reviewed the charts of patients diagnosed and treated for thalamic tumors at our institution.

Results: There were 17 patients: 12 (70\%) males and five (30\%) females. Their ages ranged from 3.5 to 12 years (avg 8.5 years). Two patients $(12 \%)$ had bi-thalamic tumors. Presenting symptoms included those of raised ICP in eight (47\%), tremor in five $(29 \%)$, contralateral weakness in three (17.6\%), hemianopia in one and seizures in one. The duration of symptoms ranged between acute onset of headache and several years history of seizures. Sixteen patients had surgery for their tumors: 13 had one surgery, two had 
two surgeries and one had three. Two patients had a stereotactic biopsy. Two patients had an endoscopic biopsy with fenestration of the intraventricular septum and CSF shunt. The pathology was low grade glioma in 10 patients (59\%), and an anaplastic astrocytoma in three $(18 \%)$. One patient each had a pilomyxoid astrocytoma, low grade mixed glioma, ganglioglioma and glioblastoma. In the low grade glioma group three patients $(27 \%)$ had progression of disease and had further treatment with chemotherapy and/or radiotherapy. One of these patients de-differentiated to an anaplastic astrocytoma and died four months later. In the high grade astrocytoma group, all patients had poor outcome: one patient died five months after diagnosis, two were discharged on palliative treatment three months after diagnosis. The forth patient died of intratumoral bleed two weeks after presentation, and had a glioblastoma on postmortem exam.

Conclusions: Pediatric thalamic tumors are predominantly low grade gliomas and generally carry a good prognosis. The pathology, however, is mixed, and those with high grade gliomas carry a poor prognosis with a few months survival. Biopsy is therefore an integral component of management, which may be followed by surgical resection and/or adjuvant therapy.

\section{SPINE}

\section{P-140}

\section{Thoracoscopic spinal surgery for thoracic spine tumor} resection

\section{K Reddy, J Miller, A Algird*, (Hamilton, ON)}

Background: Anterior thoracoscopic approach for paraspinal and spinal tumors was reported in the literature with promising results of less invasive method over the standard thoracotomy.

Objectives: To report on the use of thoracoscopy for treatment of paraspinal tumors.

Methods: Retrospective study of two patients who had anterior thoracoscopy for resection of dumbbell shape tumors. CT scan and MRI were done as preoperative evaluation. Three months outcome follow-up. First patient went for thoracoscopic biopsy of paraspinal tumor followed by thoracoscopic total resection in two years. Second patient had total resection of paraspinal tumor

Results: First patient had no complications with the tumor biopsy, but a bronchopleural fistula developed from his second thoracoscopy for total resection, which required open thoracotomy for fistula repair. Second patient had good outcome. Total resection of the tumors was confirmed by follow-up imaging.

Conclusions: Promising procedure in term of risks, less hospitalization and patient comfort after the surgery. It is still considered a new approach, which need learning surgical skills. Further prospective studies with long follow-up and larger volume of patients will be required before we can determine the real benefits of thoracoscopic spine surgery

\section{P-141}

The use of $\mathrm{C} 1$ lateral mass screws in complex cervical spine surgery

T Gunnarsson*, E Massicotte, P Govender, M Fehlings, (Toronto, $O N)$

Background: There are a number of different methods to treat atlantoaxial instability of which $\mathrm{C} 1-\mathrm{C} 2$ transarticular screws is considered to be the most biomechanically stable fixation. The procedure is technically demanding and is not feasible in a number of patients due to unfavorable anatomy. Recently, an alternative method was described using polyaxial screws inserted into the lateral mass of $\mathrm{C} 1$ and pars interarticularis of $\mathrm{C} 2$ connected by rods.

Methods: We report our initial experience with this technique in five patients where the insertion of $\mathrm{C} 1-\mathrm{C} 2$ transarticular screws was not possible.

Results: Two patients with sarcoma underwent tumor removal where the $\mathrm{C} 1$ lateral mass screws were included in a long complex construct. One patient had severe degenerative cervical spondylosis with central cord syndrome which required decompression and fixation from C1-T1. Two patients with odontoid fractures were treated with $\mathrm{C} 1-\mathrm{C} 2$ fixation. The follow-up period was up to 10 months and no instrument failure was seen.

Conclusion: In cases where fixation of $\mathrm{C} 1-\mathrm{C} 2$ is indicated and insertion of $\mathrm{C} 1-\mathrm{C} 2$ transarticular screws is not feasible, instrumentation with polyaxial screws into the lateral mass of $\mathrm{C} 1$ and pars interarticularis of $\mathrm{C} 2$ is a safe and biomechanically effective alternative.

\section{P-142}

The use of recombinant human bone morphogenetic protein-7 as an adjuvant in spinal fusion surgery

P Govender*, Y Rampersaud, L Rickards, M Fehlings, (Toronto, $O N)$

Background: Non-human studies show that osteogenic protein-1 (OP-1), a bone morphogenetic protein (BMP-7), enhances spinal fusion but its use in humans is still being evaluated.

Methods: We prospectively evaluated 11 high-risk patients who required spinal fusion. OP-1 was mixed with autogenous bone and placed at the fusion site during surgery. Questionnaire based surveys were used for patient demographics. The SF-36 questionnaire and Oswestry Disability Index (ODI) were used pre- and postoperatively. All patients had routine spinal x-rays during followup.

Results: Patients ages (five females and six males) ranged from 21 to 75 years with average height of 1.63 metres and weight of 78.36 kilograms. Risk factors included mucopolysaccharide syndrome; adrenal insufficiency; rheumatoid arthritis; obesity and heavy smoking. Surgery (five cervical spine and six lumber spine) was uneventful in all. The postoperative follow-up period ranged from six to 18 months. The average ODI scores decreased from moderate and severe disability preoperatively to mild disability postoperatively. The SF-36 survey showed an overall improved mental and physical health score. None of the patients had fusion failure after three months.

Conclusions: OP-1 putty appears to be clinically safe for use in 
spinal fusion with bony fusion possible even in patients with adverse medical risk factors.

\section{P-143}

\section{Spontaneous spinal epidural hematoma during pregnancy}

M Kelly*, C Beavis, V Sadanand, S Hattingh, (Saskatoon, SK)

Background: Spontaneous spinal epidural hematoma is a rare phenomenon with no distinct etiology. Spontaneous spinal epidural hematoma during pregnancy is extremely rare. We present what we believe to be the fourth reported case of spontaneous spinal epidural hematoma associated with pregnancy.

Methods: A 31-year-old female presented with acute onset of paraplegia at 32 weeks of pregnancy. Lower extremity motor function and bowel and bladder function was absent. She had a T2 sensory level to pinprick with preservation of posterior column function. Magnetic resonance imaging showed an acute epidural hematoma posterior to the thoracic spinal cord between the second and fourth thoracic vertebrae.

Results: The patient was taken to the operating room were her child was delivered by caesarean section. She then underwent a posterior laminectomy and evacuation of a spinal epidural hematoma. She had no coagulopathy. Follow-up selective spinal angiography was negative for any vascular malformation. The patient was able to regain bowel and bladder function by three months and was ambulating independently six months postoperatively.

Conclusions: Spontaneous spinal epidural hematoma is a rare phenomenon. Rapid diagnosis and surgical intervention may improve the eventual clinical result. We present what we believe to be the fourth reported case associated with pregnancy.

\section{P-144}

\section{Hematological abnormalities in spinal cord injured individuals} during the acute post-traumatic period

\section{J Furlan*, A Krassioukov, M Fehlings, (Toronto, ON)}

Background: Since the kidneys, spleen, bone marrow and surrounding tissues are regulated by autonomic nervous system, we hypothesized that hematological changes would occur after spinal cord injury (SCI).

Methods: All consecutive individuals with acute cervical SCI and no other major trauma admitted to a university hospital between 1998-2000 were retrospectively analyzed.

Results: There were 26 individuals (20M, 6F, ages 17-89 years, mean $=59.5$ ) who underwent hematological testing within first three postinjury weeks. Reduced hemoglobin levels occurred in $84.6 \%$, $96.1 \%, 100 \%$ individuals within first, second, third post-SCI weeks, respectively. While there were no significant differences in the hemoglobin levels between severe (ASIA A\&B) and mild SCI (C\&D), hemoglobin levels were lower in severe than mild SCI individuals within first three post-SCI weeks. Leukocytosis arose in $46.1 \%, 73.1 \%, 52.4 \%$ individuals within three post-SCI weeks. Lymphopenia affected $80.8 \%, 25 \%, 66.7 \%$ individuals within three post-SCI weeks. Thrombocytopenia occurred in $30.8 \%, 3.8 \%, 0 \%$, whereas thrombocytosis arose in $0 \%, 3.8 \%, 3.8 \%$ individuals within three post-SCI weeks. Lymphocyte, leukocyte, and platelet concentrations were unaffected by severity of SCI.
Conclusions: Hematological changes are frequent and may reflect autonomic dysfunction that affect haemopoietic system after SCI. Further investigation is essential to understand these changes since they may affect survival and quality-of-life of SCI individuals.

\section{P-145}

Precision of spinal neuronavigation for lumbosacral pedicle screw fixation in $\mathbf{3 0}$ consecutive cases

\section{$B$ Goulet *, J Pelletier, (Montreal, QC)}

Background: Neuronavigation is technically more difficult to use for spine than for brain. The registration can only be done on the posterior part of the spine. The length of procedure has discouraged users. Nonetheless the precision for pedicular screw fixation has been superior using this technique.

Methods: We implanted 179 lumbosacral pedicle screws in 30 patients using preoperative CT scan based neuronavigation (SNN). All patients had a postoperative CT scan. The medial, lateral, superior and inferior pedicles were assessed for minor errors (0.1$2.0 \mathrm{~mm})$ and major errors $(>2.1 \mathrm{~mm})$. The length of the screws was measured in relation to the anterior cortex. Too long screws: $>1.1 \mathrm{~mm}$ past the cortex for L1-5 and $>5.1 \mathrm{~mm}$ for $\mathrm{S} 1$. Short screws: $>10.1 \mathrm{~mm}$ at $\mathrm{L} 1-5$ and $<0 \mathrm{~mm}$ at $\mathrm{S} 1$ from the anterior cortex.

Results: We obtained 93\% excellent screws, $6 \%$ minor and $1 \%$ major errors. None of the errors had clinical relevance. Most errors occurred in the lateral wall of L5 followed by the inferior wall. The length was too long in 3\% and to short in $7 \%$ of the screws without clinical significance. We had no vascular or neurological injuries.

Conclusions: We had $93 \%$ of perfect pedicle screws using neuronavigation, showing superiority over conventional methods.

\section{STROKE}

\section{P-146}

Postpartum headache secondary to combination of cerebral venous sinus thrombosis and epidural-induced spinal fluid leak

S Subramaniam*, A Kirton, $R$ Sevick, G Klein, (Calgary, AB)

Background: Both cerebral venous sinus thrombosis (CVST) and spinal fluid leak are increased postpartum. Their occurrence in combination, however, has rarely been reported. Epidemiological data would suggest that neither are a risk factor for the other. However, potentially dangerous delays in the recognition of CVST has been suggested to occur when headaches due to a combination of both causes are attributed only to spinal fluid leak.

Methods: Case report.

Results: We report a 28-year-old, previously healthy, multiparous woman who presented with two distinct varieties of headache one week following an uncomplicated pregnancy. One was consistent with an epidural-induced spinal fluid leak headache. When this headache improved with blood patch, she was discharged home. Only on returning 48 hours later with worsening of her second variety of headache, which had never improved, was a noncontrast CT scan performed in the emergency room and was suggestive of a subtle thickening of the superior saggital sinus. Magnetic resonance venogram confirmed the presence of superior sagittal sinus thrombosis and the patient was subsequently admitted and anticoagulated. 
Conclusions: CVST and epidural-induced spinal fluid leak can occur in combination and headaches felt to be consistent with one should not preclude consideration of the other.

\section{P-147}

\section{Acute migraine attacks misdiagnosed as transient ischemic attacks}

\section{N Vujovic-Zotovic*, DJ Sahlas, JW Norris, (Toronto, ON)}

Background: Migraine attacks may be accompanied by a variety of transient neurological symptoms, but when occurring in later life may be misdiagnosed as acute ischemic events seen in other forms of cerebrovascular disease.

Methods: We prospectively evaluated all patients referred to our Stroke Prevention Clinic over three years, with the diagnosis of TIA or stroke. All patients had brain scanning by CT or MRI, carotid Doppler evaluation, echocardiography and, where indicated, cerebral angiography.

Results: Following outpatient investigation of 1482 patients, 118 (8\%) had a final diagnosis of migraine. 106 of these presented as TIAs, 52 with visual symptoms only, with a typical migrainous pattern, 17 with neurological symptoms only, with characteristic migrainous march, and 37 patients with a mixture of visual and neurological symptoms.

Conclusions: Migraine commonly simulates TIAs, but can be distinguished by its clinical features combined with otherwise negative radiological and laboratory investigations.

\section{P-148}

\section{Improved detection of microbubble signals using power $M$ mode Doppler}

Maher Saqqur*, Naeem Dean, Abdul Salam, Ashfaq Shuaib, Andrew Demchuk, (Edmonton, AB); Marcia Schebel, (Calgary, AB)

Background: Power motion mode (PMD) TCD is a new multigated technique that may simplify and enhance detection of embolus. We developed stringent criteria for emboli detection using PMD TCD since standard criteria do not exist. Using a novel stringent embolus definition for PMD embolus counting we performed a blinded comparison of transcranial PMD with standard single gate spectral TCD in TCD bubble study patients.

Methods: Patients with right to left shunt (RLSh) as detected with standard TCD (positive bubble study) were selected for this comparison study. Ninth International Cerebral Hemodynamic Symposium embolus counting criteria for spectral TCD were used. We defined new novel PMD criteria for detecting emboli signature on the PMD display as follows: 1) signature at least $3 \mathrm{~dB}$ higher than the highest spontaneous PMD display of background blood flow signal; 2) embolic signature reflects motion in one direction at a minimum spatial extent of $7.5 \mathrm{~mm}$ and a minimum temporal extent of 30 milliseconds; 3) the single embolus must traverse a specific depth determined by the highest intensity of the insonated artery. Each study was independently and blindly assessed for microbubble signals (MBS) count on either spectral TCD or PMD.

Results: 38 patients studied, mean age $44 \pm 14,50 \%$ male, median time of TCD bubble test after stroke onset 11 days. Median number of MBS identified per hemisphere insonated at rest was $(n=58)$ : spectral TCD 4 versus PMD 4 (MCA only) ( $\mathrm{p}$ value is NS); spectrogral TCD 4 versus PMD 6 (MCA+ACA) (p value 0.001). The median number of MBS identified per hemisphere with valsalva maneuver (VM) was $(n=36)$ : spectrogram 20 versus PMD 22 (MCA only) (p value NS); spectrogram TCD 20 versus PMD 32 (MCA+ACA) (p value less 0.001).

Conclusions: When compared with single gate spectral TCD, the multi-gated transcranial PMD was more sensitive in detecting MBS with higher median MBS counts by identifying ACA as well as MCA emboli. Pitfalls of overcounting emboli with PMD can be avoided by following stringent criteria.

\section{P-149}

\section{Power M mode transcranial Doppler (PMD/TCD) criteria for} angiographic proven arterial occlusion

Maher Saqqur*, Ashfaq Shuaib, (Edmonton, AB); Marcia Schebel, Andrea Krol, Michael Hill, Andrew Demchuk, (Calgary, AB)

Background: Stringent transcranial Doppler criteria for diagnosing occlusion are needed to better apply TCD in the acute stroke setting. At three experienced stroke centers we performed TCD examination for patients with symptoms of cerebral ischemia who underwent gold standard digital subtraction angiography (DSA). We used standardized insonation protocols and a novel TCD technology power M mode Doppler (PMD) (Spencer Technologies Inc) in each case. Several PMD flow findings were evaluated to predict anterior circulation occlusions as assessed by receiver operating characteristic (ROC) analysis.

Methods: We studied 74 PMD examinations prior to or immediately after DSA. We analyzed mean flow velocity (MFV), pulsatility index (PI), and power $\mathrm{M}$ mode resistance signature (absent, high or low) in symptomatic MCA, ACA, PCA, and contralateral (c/lat) MCA. M mode signal was used to immediately locate and obtain spectrographic recordings at the depth of occlusion. Proximal middle cerebral artery depths of 55-65 and 45$55 \mathrm{~mm}$ and all ACA (60-75mm) and PCA (55-75mm) depths were analyzed. The maximum mean flow velocity at each depth was used to calculate mean flow velocity ratios.

Results: Affected / contralateral MCAMFVratio, affected MCA/ ipsilateral ACA MFV ratio, and PMD resistance flow pattern were found to be the best predictor of anterior circulation occlusion. The aMCA/cMCAratio was most robust at 55-65 mm depths (area under receiver operated curve 0.7983 ) but not at $45-55 \mathrm{~mm}$ depths and the aMCA/iACAand aMCA/iPCAratios were highly correlated. Based on the flow findings which predicted anterior circulation occlusion we developed a 10 points score system that consisted of affected/ unaffected MCA ratio, affected MCA/ipsilateral ACA MFV ratio, and PMD resistance pattern which demonstrated excellent specificity.

Conclusions: When applied in the acute stroke setting a combination of mean flow velocity ratios and power $\mathrm{M}$ mode Doppler can be used to predict anterior circulation occlusion. Very high specificity can be achieved when these flow findings parameters are combined in a score. Further refinement by exact site occlusion and validation is needed. 
P-150

\section{Neuroprotective effects of dipyridamole as compared with standard antiplatelet treatment in an embolic ischemic stroke model}

Samer Aldandashi*, Raza Noor, Asim Raja, Chen xu Wang, Ashfaq Shuaib, (Edmonton, AB)

Background: The primary role of antiplatelet therapy is to prevent stroke in high-risk individuals. The purpose of this study was to test the protective effects of varying doses of two antiplatelets (dipyridamole and acetylsalicylic acid (aspirin)) using an embolic model of focal ischemia in rats.

Methods: Five groups of rats were given the following treatment orally twice daily four days before and two days after stroke: placebo (simple syrup) dipyridamole $80 \mathrm{mg} / \mathrm{kg}$, dipyridamole $200 \mathrm{mg} / \mathrm{kg}$, aspirin $0.625 \mathrm{mg} / \mathrm{kg}$, and aspirin $5 \mathrm{mg} / \mathrm{kg}$. Focal ischemia model was used and infarction volume measured by staining with 2,3,5-triphenyltetrazolium chloride and calculating the size of the infarction 48 hours after the insult.

Results: The infarction volume for the placebo group was $56.60 \% \pm 3.85 \%$. In the group treated with dipyridamole $80 \mathrm{mg} / \mathrm{kg}$ the volume of infarction was $48.42 \% \pm 4.76 \%(p<0.05)$. In the dipyridamole $200 \mathrm{mg} / \mathrm{kg}$ group the infarction volume was $27.88 \% \pm$ $4.15 \%$ ( $\mathrm{p}=7.38 \times 10-6)$. In the aspirin $0.625 \mathrm{mg} / \mathrm{kg}$ group it was $37.52 \% \pm 4.10 \%$. Finally in the aspirin $5 \mathrm{mg} / \mathrm{kg}$ group it was $42.03 \%$ \pm 3.61 .

Conclusion: There is a significant dose-dependent reduction in the volume of cerebral infarction with dipyridamole and a dose independent reduction by aspirin. Our data suggests that a higher dose of antithrombotic therapy with dipyridamole offer best neuroprotection.

\section{P-151}

\section{No more musical beds: an integrated stroke unit}

\section{W Oczkowski*, L Gould, C Martin, A Smith, (Hamilton, ON)}

Background: Clustering patients with stroke and care provided by a stroke team improves outcomes. Rehabilitation should start on day 1 . We have opened a unique stroke unit that combines acute care and rehabilitation.

Methods: 15 acute care stroke beds were combined with 16 rehab stroke beds on the same medical ward. The acute and rehab components of care share a common medical and health care team, and physical resources. Staff have or are expected to develop skills in caring for stroke patients in both the acute and rehab phases of the care continuum. The rehab patients have access to advanced diagnostics, consultation, and treatment.

Results: Patients are accepted to rehab early and the length of stay in acute care is decreased. Patients can continue to receive their diagnostic work up, and receive specialized acute stroke treatment while in rehab. Many patients with complex medical issues can start rehabilitation early and few return into acute care because of illness.

Conclusions: The integrated stroke unit provides a single unit for care across the continuum, and avoids the musical beds approach to care.
P-152

\section{Tolerability of Aggrenox $^{\mathrm{TM}}$ in a community-based setting}

Harris Lari*, Thomas Jeerakathil, Michael Mouradian, Ashfaq Shuaib, (Edmonton, AB)

Background: Although proven to be highly effective in stroke prevention, clinical practice experience suggests that many patients do not tolerate Aggrenox ${ }^{\mathrm{TM}}$ because of side effects. Studies are required to determine the tolerability of Aggrenox ${ }^{\mathrm{TM}}$ in real-practice settings to determine the medication's importance in stroke prevention in the community.

Methods: This is a retrospective cohort study with telephone follow-up in the setting of a community-based stroke prevention clinic. Charts from September 2001 to December 2002 were reviewed and 133 consecutive patients identified who were prescribed Aggrenox ${ }^{\mathrm{TM}}$ after stroke or TIA. Standardized telephone interview is being conducted between two months to one year post clinic visit to determine the discontinuation rate of Aggrenox $^{\mathrm{TM}}$ and reasons for discontinuation at one month post initiation of therapy.

Results: The proportion of subjects discontinuing therapy at one month post initiation will be reported, as will the reasons for discontinuation. Logistic regression will be used to determine patient characteristics predictive of discontinuation.

Conclusions: We hope to provide important data on the tolerability of Aggrenox ${ }^{\mathrm{TM}}$ in a real-practice setting and to provide perspective on the medication's importance in stroke prevention in the community.

\section{P-153}

\section{Diagnosing vertebral artery dissection in the Emergency Department}

C Phan*, B Stewart, (Edmonton, AB)

Background: Vertebral artery dissection is a leading cause of stroke and subsequent neurological deficits in younger patients. Studies have been done in the past to identify the possible causes, risk factors, clinical signs and symptoms in order to facilitate diagnosis and timely treatment. However, vertebral artery dissection remains a commonly misdiagnosed presentation in the Emergency Room (ER).

Methods: This is a retrospective case study of three patients, all female, age ranging from 31 to 50-year-old. We studied ER records as well as assessments by the consulting neurologist.

Results: The patients presented to the ER with symptoms ranging from neck pain, neck stiffness dizziness, vertigo, slurred speech visual, sensory and motor changes. Predisposing events include cervical chiropractic manipulation, self-manipulation of neck, and rapid head movement respectively. Clinical findings and head CT were all negative. Emergency physicians missed the diagnosis of vertebral artery dissection on initial presentation and all three patients were later on found to have vertebral artery dissection with brainstem and cerebellar infarcts.

Conclusions: Patients with history of chiropractic cervical manipulation, rapid neck movements, or cervical trauma presenting with symptoms of headache, neck pain, dizziness/vertigo, visual changes, sensory and/or motor changes should be investigated thoroughly for vertebral artery dissection. 


\section{VASCULAR NeURosUrGery}

\section{P-154}

\section{Endovascular treatment of cerebral aneurysms in children}

$R$ Yahya*, D Armstrong, K ter Brugge, J Drake, (Toronto, ON)

Background: Aneurysms are rare in children, often in unusual locations, and frequently complex. They are, however, amenable to treatment by endovascular techniques.

Objective: The purpose of this report is to present four cases of cerebral aneurysms that were treated by endovascular techniques.

Methods and results: Five patients with cerebral aneurysm were treated endovascularly at our hospital. Two of these patients (ages nine and seven years) had intracavernous ICA aneurysms and presented with diplopia. They underwent test occlusion of their ICA for 20 minutes followed by definitive balloon occlusion which was well tolerated clinically, resulting in obliteration of the aneurysms radiologically. The third patient (age seven months) had a ruptured complex ACA aneurysm and he underwent successful treatment with coil embolization resulting in occlusion of the aneurysm sac as well as the A1 and the A2 vessels on that site. The fourth patient had a thalamic tumor and an ICAaneurysm and was treated with partial embolization using coils. The fifth patient presented with ruptured mycotic aneurysm of the left superior cerebellar artery. He underwent coiling prior to posterior fossa decompression.

Conclusions: Endovascular techniques can be useful in the treatment of children with cerebral aneurysms. In selected patients, this may eliminate the need for high risk surgical procedures. Longer follow-up is needed to assess the long term results.

\section{P-155}

Intractable status epilepticus post carotid endarterectomy - a clinical presentation of cerebral hyperperfusion syndrome

\section{$S$ Yip*, H Feldman, (Vancouver, BC)}

Background: Cerebral hyperperfusion syndrome (CHS) is an uncommon but potentially serious adverse event following carotid endarterectomy (CEA) or endovascular stenting of stenotic carotid arteries. Its clinical presentation ranges from headache, confusion, and seizure, to intracranial hemorrhage. The etiology of CHS has been attributed to impaired cerebrovascular autoregulation in conjunction to revascularization of a stenotic carotid artery while other studies pointed to cerebral embolization.

Results: We describe a patient with intractable left hemispheric status epilepticus one week after uneventful CEA. On the day of his admission, the patient complained of dizziness and promptly collapsed. He had a tonic-clonic seizure after a right-sided focal seizure. The patient was loaded with anti-epileptic drugs. He was admitted to the ICU and was placed on propofol to induce burst suppression coma. Serial CT scans revealed no intracerebral abnormalities. The patient was also heparinized secondary to finding of a small distal intimal flap in the left ICA on CT angiogram. Despite maximal medical therapy, the patient showed no clinical improvement. Care was withdrawn after discussion with the family.

Discussion: We report a case of intractable status epilepticus post-CEAand will discuss detail pertinent to this case as well as the relevant literature on CHS.

\section{P-156}

Multimodality management of spinal perimedullary fistulas

H Jain*, M Tymianski, $R$ Willinsky, W Montanera, K ter Brugge, MC Wallace, (Toronto, ON)

Introduction: Spinal perimedullary arteriovenous fistulas are rare lesions, distinct from spinal cord AVMs, which can present with progressive myelopathy or subarachnoid haemorrhage. Their frequently ventral location, small caliber and tortuosity of feeding vessels make them a challenge to localize and treat. We report the experience of the Toronto Vascular Malformation group in the management of these lesions.

Methods and results: Between 1993-2003, seven patients with perimedullary fistulas were treated. Two patients presented with subarachnoid haemorrhage. Five patients presented with myelopathy, with accompanying signal changes on MRI. Five fistulae were supplied by the anterior spinal artery, one by the posterior spinal artery and one had a dual supply. Embolisation was successful as an adjunct in one out of the four in which it was attempted. All the patients underwent surgical excision of the fistula by a posterior or posterolateral approach to the relevant part of the spine with angiographic cure. In the ventrally placed lesions, division of the denticulate ligament allowed rotation of the cord to access the lesion. In two patients, intra-operative draining vein pressure measurements guided complete excision of the lesion.

Conclusions: The angio-morphological features of perimedullary arteriovenous fistulas usually preclude cure by endovascular means alone. Surgery was effective in obliterating the fistula in all cases. 


\section{INDEX}

\begin{tabular}{|c|c|c|c|c|c|c|c|}
\hline Abbott, D & H-01 & Becker, WJ & L-01 & Carmant, L & P-053 & Demchuk, Andrew & P-149 \\
\hline Abdillahi, O & P-088 & Behie, L & P-006 & Caruso, $\mathrm{M}$ & P-109 & Demeria, D & P-084 \\
\hline Abdollahi, Ali & P-096 & Behie, L & P-007 & Casey, R & P-029 & Denault, A & P-015 \\
\hline Abi Daoud, M & P-018 & Belanger, E & P-056 & Cenic, A & I-08 & Denault, A & P-016 \\
\hline Adams, C & P-027 & Belkadi, Z & P-021 & Chan, S & $\mathrm{I}-07$ & Dennis, M & $\mathrm{P}-022$ \\
\hline Adams, $\mathrm{R}$ & B-04 & Bell, RB & P-126 & Chen, Robert & & Dennis, $\mathrm{M}$ & C-08 \\
\hline Adams, $\mathrm{R}$ & P-116 & Belletrutti, M & P-031 & \multicolumn{2}{|c|}{ Herbert Jasper Prize } & Denovan-Wright, EM & P-001 \\
\hline Ahmed, SN & $\mathrm{H}-04$ & Benadellah, B & G-02 & Chen, $\mathrm{H}$ & B-08 & Derakhshan, I & $\mathrm{K}-02$ \\
\hline Ahmed, SN & P-048 & Benko, A & P-108 & Chertkow, $\mathrm{H}$ & $\mathrm{J}-06$ & DeSilva, C & P-110 \\
\hline Ahmed, SN & $\mathrm{P}-050$ & Bercovici, E & K-04 & Chertkow, $\mathrm{H}$ & $\mathrm{J}-07$ & Desjardins, A & P-116 \\
\hline Ahmed, SN & P-131 & Berger, E & P-071 & Chow, M & F-06 & Desjardins, A & P-108 \\
\hline Akagami, R & P-072 & Bergman, $\mathrm{H}$ & $\mathrm{J}-06$ & Chow, V & P-098 & Diadori, $\mathrm{P}$ & P-053 \\
\hline Aldandashi, Samer & P-150 & Berk, C & P-081 & Chow, V & P-122 & Dilenge, M-E President & 's Prize \\
\hline Alemdar, A & P-090 & Berk, C & P-091 & Christie, $\mathrm{S}$ & P-004 & Ding, $\mathrm{H}$ & B-03 \\
\hline Alexandrov, Andrei & F-01 & Berthelet, F & D-02 & Christie, S & P-014 & Dion, M-H & P-059 \\
\hline Alexandrov, Andrei & $\mathrm{F}-02$ & Betensky, R & P-118 & Christie, $\mathrm{T}$ & P-014 & Dionne, $\mathrm{M}$ & P-113 \\
\hline Algird, A & P-140 & Bhargava, R & P-135 & $\mathrm{Chu}, \mathrm{J}$ & D-04 & Dirks, Peter B & B-06 \\
\hline Al-Khatib, Y & P-026 & Black, Sandra E & P-041 & Chu, JK & L-05 & Doan, J & D-06 \\
\hline Amo, C & H-06 & Blackman, M & C-07 & Chu, JY & L-05 & Doig, G & K-07 \\
\hline Amo, C & $\mathrm{J}-01$ & Blackman, $\mathrm{M}$ & P-023 & Chung, AG & L-05 & Dong, C & P-072 \\
\hline Amo, C & $\mathrm{J}-05$ & Blackman, $\mathrm{M}$ & P-032 & Clarke, D & M-01 & Dooley, J & C-03 \\
\hline Amo, C & P-039 & Blanchet, P & P-088 & Clarke, DB & $\mathrm{I}-02$ & Dooley, J & P-018 \\
\hline Amo, C & P-052 & Blaser, S & P-022 & Clarke, Ian D & B-06 & Doucet, G & G-01 \\
\hline Andermann, F & $\mathrm{H}-02$ & Blume, W & P-046 & Clarke, L & M-01 & Drake, J & P-139 \\
\hline Andermann, F & P-038 & Blume, W & P-047 & Clarke, S & $\mathrm{P}-042$ & Drake, J & P-154 \\
\hline Angers-Loustau A & B-05 & Bock, N & B-01 & Cochrane, D & M-02 & Ducharme, ME & P-007 \\
\hline Archer, J & H-01 & Bogaars, A & P-101 & Collin, I & $\mathrm{J}-06$ & Dufour, JJ & I-01 \\
\hline Armstrong, D & P-154 & Bojanowski, MW & P-015 & Comeau, B & P-004 & Duggal, N & E-03 \\
\hline Arnold, DL & G-05 & Bojanowski, MW & P-016 & Connolly, M & P-034 & Dunham, C & H-05 \\
\hline Arnold, DL & P-092 & Bonn, Victoria E & B-06 & Constantoyannis, C & P-091 & Duquette, A & G-04 \\
\hline Aronyk, K & P-058 & Borhani-Haghighi, A & D-03 & Cortez, MA & K-08 & Duquette, $\mathrm{P}$ & $\mathrm{J}-03$ \\
\hline Arrazola, J & $\mathrm{J}-05$ & Bouchard, JP & G-04 & Cortez, MA & K-04 & Easton, AS & P-073 \\
\hline Ashby, JG & P-002 & Bouchard, R & $\mathrm{J}-07$ & Cossette, $\mathrm{P}$ & D-02 & Ebenezer, $\mathrm{S}$ & P-134 \\
\hline Ashby, JG & P-003 & Bouchentouf, $\mathrm{M}$ & G-02 & Cossette, $\mathrm{P}$ & P-053 & Ebenezer, Sudesh & P-103 \\
\hline Ashforth, R & P-010 & Brais, B & D-02 & Cossette, $\mathrm{P}$ & P-059 & Ehrensperger, E & G-05 \\
\hline Ashjazadeh, N & D-03 & Brais, B & G-04 & Cossoy, MB & P-093 & Eizenman, M & C- 08 \\
\hline Ashworth, N & H-04 & Brannan, R & P-056 & Coupal, D & P-137 & El Fahime, E & G-02 \\
\hline Asyyed, A & P-014 & Brass, $\mathrm{S}$ & P-038 & Courville, $\mathrm{J}$ & H-02 & El Fahime, E & P-129 \\
\hline Bahary, JP & P-114 & Brass, SD & P-092 & Coutts, $S$ & P-011 & El-Hakim, H & P-135 \\
\hline Baker, A & M-05 & Brass, SD & s Prize & Cramer, B & P-078 & Emery, D & P-066 \\
\hline Bakshi, Neely & P-041 & Brown, L & D-06 & Crevier, L & P-115 & Engelbrecht, A & P-113 \\
\hline Ball, M & J-08 & Brownell, AKW & P-126 & Crocker, S & M-06 & Evoy, F & P-108 \\
\hline Bangash, M & P-113 & Brownstone, $\mathrm{R}$ & M-01 & Currie, W & P-004 & Fazl, M & K-03 \\
\hline Banigesh, A & M-03 & Brownstone, $\mathrm{R}$ & P-081 & Dalton, $\mathrm{P}$ & E-01 & Federico, $\mathrm{P}$ & H-01 \\
\hline Banwell, B & $\mathrm{C}-06$ & Brownstone, $\mathrm{R}$ & P-091 & Darsaut, $\mathrm{T}$ & P-074 & Fehlings, $\mathrm{M}$ & E-02 \\
\hline Banwell, B & P-025 & Brunner, J & P-097 & Darseault, T & P-075 & Fehlings, $\mathrm{M}$ & E-04 \\
\hline Bar-Or, A & P-093 & Bulman, D & D-07 & Darvesh, S & P-040 & Fehlings, $\mathrm{M}$ & E-05 \\
\hline Bar-Or, A & P-092 & Busche, K & P-020 & De Leon, J & L-07 & Fehlings, $\mathrm{M}$ & E-06 \\
\hline Barron, J & P-076 & Bussiere, Miguel & P-044 & Dean, Naeem & P-148 & Fehlings, $\mathrm{M}$ & P-141 \\
\hline Barron, J & P-107 & Cairncross, G & P-118 & Decarie, J-C & $\mathrm{P}-053$ & Fehlings, $\mathrm{M}$ & P-142 \\
\hline Bartlett, G & G-03 & Caldwell, J & P-117 & Del Maestro RF & B-05 & Fehlings, $\mathrm{M}$ & P-144 \\
\hline Bartlett, P & P-107 & Calleja, Sergio & F-01 & Del Maestro, RF & P-104 & Feldman, $\mathrm{H}$ & P-155 \\
\hline Bastos, A & P-043 & Camfield, C & C-05 & DeMarchi, R & E-01 & Fernandez, A & H-06 \\
\hline Beauprie, I & P-081 & Camfield, P & C-05 & Demchuk, Andrew & F-01 & Fernandez, A & $\mathrm{J}-01$ \\
\hline Beauvais, C & P-088 & Camicioli, R & $\mathrm{J}-08$ & Demchuk, Andrew & F-02 & Fernandez, A & $\mathrm{J}-05$ \\
\hline Beavis, C & P-143 & Caramanos, Z Preside & 's Prize & Demchuk, Andrew & P-148 & Fernandez, A & P-039 \\
\hline
\end{tabular}




\begin{tabular}{|c|c|c|c|c|c|c|c|}
\hline Fernandez, A & P-052 & Guha, A & B-01 & Hooge, J & P-042 & Kim, Ji Soo & D-08 \\
\hline Fernandez, S & J-01 & Guha, A & B-03 & Hoskinson, M & P-048 & Kim, JS & P-120 \\
\hline Field, TS & $\mathrm{F}-05$ & Guha, A & B-07 & Houlden, D & K-03 & Kim, K & P-062 \\
\hline Findlay, J Max & A-05 & Guha, A & M-07 & $\mathrm{Hu}, \mathrm{W}$ & P-011 & Kim, MJ & P-062 \\
\hline Findlay, JM & P-073 & Guha, A & P-080 & Huber-Okrainec, J & $\mathrm{P}-022$ & Kingstone, $\mathrm{M}$ & P-124 \\
\hline Fladeland, D & F-03 & Guha, A & P-100 & Huh, L & P-033 & Kirk, A & P-063 \\
\hline Fleetwood, I & P-014 & Guha, A & P-102 & Huh, L & P-034 & Kirkwood, A & P-110 \\
\hline Fleetwood, I & P-077 & Guha, A & P-117 & Hunter, G & P-084 & Kirton, A & P-020 \\
\hline Fleetwood, I & I-02 & Guimond, $\mathrm{CH}$ & $\mathrm{P}-025$ & Huntsman, R & P-030 & Kirton, A & P-146 \\
\hline Fleetwood, IG & P-113 & Guiot, M-C & P-099 & Hussain, S & P-125 & Kirton, A & P-019 \\
\hline Forsyth, P & P-118 & Gul, S & D-05 & Hutchinson, Z & P-046 & Kiss, Z & D-06 \\
\hline Fortin, D & B-04 & Gunnarsson, $\mathrm{T}$ & E-02 & Hutchinson, Z & P-047 & Klein, G & P-146 \\
\hline Fortin, D & P-116 & Gunnarsson, $\mathrm{T}$ & P-083 & Hwang, Jeong-Min & P-067 & Kole, M & P-068 \\
\hline Fortin, D & P-108 & Gunnarsson, $\mathrm{T}$ & P-141 & Iida, Koji & P-054 & Koles, ZJ & H-03 \\
\hline Fortin, MA & P-114 & Gupta, M & P-135 & Ilton, $\mathrm{S}$ & M-06 & Kolyvas, G & I-02 \\
\hline Fourney, D & P-097 & Hache, N & P-076 & Innes, $\mathrm{M}$ & P-024 & Kozey-Rogers, M & $\mathrm{I}-07$ \\
\hline Furlan, J & E-04 & Hahn, C & C-06 & Jacka, M & P-066 & Kozey-Rogers, M & P-087 \\
\hline Furlan, J & P-144 & Hall, C & P-105 & Jacka, M & P-075 & Krassioukov, A & E-02 \\
\hline Gagnon, A & L-07 & Hallaji, Zahra & P-121 & Jacka, Michael J & A-05 & Krassioukov, A & E-04 \\
\hline Gagnon, Sophie & P-132 & Hamilton, M & P-032 & Jackson, G & H-01 & Krassioukov, A & P-144 \\
\hline Gallagher, C & M-04 & Hamilton, $\mathrm{M}$ & P-087 & Jacob, J & P-076 & Krol, Andrea & P-149 \\
\hline Gallagher, C & P-005 & Hamiwka, L & C-07 & Jacob, J & P-078 & Kumar, K & P-084 \\
\hline Gallez, A & P-116 & Hamiwka, L & P-023 & Jain, H & A-02 & Lad, T & P-119 \\
\hline Gao, Fuqiang & P-041 & Hamiwka, L & P-032 & Jain, H & P-156 & Lafreniere, J-F & P-129 \\
\hline Gaul, P & P-029 & Hammond, R & P-064 & James, CD & B-07 & Lafrenierre, JP & G-02 \\
\hline Genge, A & G-05 & Hammond, R & P-111 & James, T & P-080 & Lah, S & L-03 \\
\hline Gentili, F & A-08 & Han, F & D-07 & Jassal, R & P-125 & Landesman, B & P-035 \\
\hline George, D & P-020 & Hanson, $\mathrm{T}$ & D-06 & Javaheri, Jamshid & P-096 & Lang, A & D-07 \\
\hline Germain, E & P-109 & $\mathrm{Hao}, \mathrm{C}$ & P-073 & Jeerakathil, Thomas & P-152 & Lang, A Herbert Jasp & er Prize \\
\hline Gil, $P$ & P-039 & Harrison, E & L-02 & Jensen, $\mathrm{P}$ & P-107 & Langlois, M-A & G-01 \\
\hline Girard, F & P-015 & Hattingh, S & P-143 & Jirsch, J & G-05 & Lapierre, Y Presiden & 's Prize \\
\hline Glass, H & P-024 & Hattingh, S & P-105 & Johnston, W & P-125 & Lari, Harris & P-152 \\
\hline Gokaslan, Z & P-097 & Haw, C & P-017 & Juurlink, B & P-105 & Lary, A & P-058 \\
\hline Gorassini, D & P-111 & Hawkins, Cynthia & B-06 & Kachur, E & P-118 & Lau, N & B-03 \\
\hline Gordon, K & C-03 & Hayat Khan, S & P-105 & Kalia, L & P-094 & Lavigne, $\mathrm{G}$ & P-088 \\
\hline Gordon, $\mathrm{K}$ & P-018 & Hebb, MO & P-001 & Kallos, M & P-006 & Lawson, J & F-03 \\
\hline Gould, L & P-151 & Hellmann, J & C-06 & Kalra, S & G-05 & Leblanc, R & P-099 \\
\hline Goulet, B & P-145 & Hendson, $\mathrm{G}$ & P-034 & Kaplan, D & P-100 & Lee, D & P-118 \\
\hline Goupil, D & $\mathrm{J}-07$ & Henry, M & P-075 & Kashmere, J & P-066 & Lee, KW & P-120 \\
\hline Govender, $\mathrm{P}$ & P-141 & Hentschel, S & A-01 & Kass, $\mathrm{M}$ & A-03 & Leibovitch, Farrell S & P-041 \\
\hline Govender, P & P-142 & Heran, N & A-01 & Kaufmann, AM & A-03 & Levin, $\mathrm{S}$ & P-046 \\
\hline Gowrishankar, M & P-075 & Heran, $N$ & M-02 & Kaufmann, AM & P-086 & Levoie, J & P-100 \\
\hline Goyal, M & P-009 & Hessler, R & P-026 & Kaufmann, AM & I-04 & Lewis, R & P-105 \\
\hline Goyal, M & P-124 & Hicdonmez, T & M-02 & Kaufmann, AM & $\mathrm{I}-05$ & Lewis, $\mathrm{T}$ & F-03 \\
\hline Griebel, R & P-098 & Hill, M & P-011 & Kaufmann, AM & I-07 & Lilge, L & P-101 \\
\hline Griebel, R & P-137 & Hill, MD & F-05 & Kaufmann, AM & P-085 & Lillakas, L & C-08 \\
\hline Griebel, R & P-138 & Hill, MD & P-019 & Kaufmann, AM & P-087 & Liu, Y & E-06 \\
\hline Griebel, R & P-105 & Hill, Michael & F-01 & Kaye, J & J-08 & Logan, W & P-028 \\
\hline Grimes, D & D-07 & Hill, Michael & P-149 & Keene, D & P-056 & Lortie, A & P-053 \\
\hline Grondin, RT & P-010 & Hill, R & I-02 & Kelly, M & P-137 & Louis, D & P-118 \\
\hline Grondin, RT & P-112 & Hillman, J & P-083 & Kelly, M & P-138 & Louw, DF & B-02 \\
\hline Gross, D & P-057 & Ho, A & P-029 & Kelly, M & P-143 & Louw, DW & $\mathrm{I}-03$ \\
\hline Gross, DW & $\mathrm{H}-03$ & Hogan, $M$ & P-008 & Kennedy, J & P-019 & Lownie, $\mathrm{S}$ & P-068 \\
\hline Gross, DW & H-04 & Hogan, M & P-009 & Kerr, R & B-08 & Lowry, $\mathrm{N}$ & P-031 \\
\hline Gross, DW & P-043 & Hogan, MJ & P-124 & Keyes, R & P-042 & Lowry, N & P-036 \\
\hline Gross, DW & P-048 & Holness, R & M-01 & Keyes, R & P-045 & Lozano AM Herbert Jas & er Prize \\
\hline Gross, DW & P-066 & Holowka, Stepanie & P-054 & Khan, S & M-03 & Lozano, AM & P-001 \\
\hline Guberman, A & P-008 & Honey, C & P-091 & Khan, S & P-070 & Lum, C & P-009 \\
\hline Gubitz, G & L-02 & Honish, Colin & F-04 & Khashab, M & P-069 & Lum, C & P-124 \\
\hline
\end{tabular}


LE JOURNAL CANADIEN DES SCIENCES NEUROLOGIQUES

\begin{tabular}{|c|c|c|c|c|c|c|c|}
\hline Lye, T & P-087 & Mendez, I & P-090 & Ogunyemi, A & P-049 & Quesney, F & P-039 \\
\hline Macaulay, RJB & P-113 & Miabi, Zinat & A-06 & Okada, Y & P-118 & Quesney, F & P-052 \\
\hline Macaulay, RJB & P-093 & Miabi, Zinat & $\mathrm{P}-123$ & Onochie, $\mathrm{C}$ & $\mathrm{P}-111$ & Quigley, D & H-04 \\
\hline Macdonald, D & P-110 & Micallef, J & P-117 & Ortiz, $\mathrm{T}$ & $\mathrm{H}-06$ & Quigley, D & P-048 \\
\hline MacGuire, C & P-048 & Midha, R & K-03 & Ortiz, T & $\mathrm{J}-01$ & Quigley, D & P-050 \\
\hline Maestu, F & H-06 & Midia, Mehran & A-06 & Ortiz, T & $\mathrm{J}-05$ & Quinn, J & $\mathrm{J}-08$ \\
\hline Maestu, F & $\mathrm{J}-01$ & Midia, Mehran & P-123 & Ortiz, T & P-039 & Rabin, D & P-012 \\
\hline Maestu, F & $\mathrm{J}-05$ & Midia, Ramin & A-06 & Ortiz, T & P-052 & Rachid, Zerrouki & L-06 \\
\hline Maestu, F & P-039 & Midia, Ramin & P-123 & Otsubo, Hiroshi & P-054 & Ragazonni, A & K-07 \\
\hline Maestu, F & P-052 & Miller, J & P-140 & Pandita, A & B-07 & Rahey, S & $\mathrm{P}-055$ \\
\hline Magnusson, JE & L-01 & Miller, W & P-009 & \multicolumn{2}{|c|}{ Paradiso G Herbert Jasper Prize } & Raja, Asim & P-150 \\
\hline Mah, J & P-019 & Miller, W & $\mathrm{P}-124$ & Park, SH & P-120 & Rampersaud, Y & P-142 \\
\hline Mah, J & P-026 & Mills, $\mathrm{P}$ & G-02 & Parr, A & A-04 & Ramsay, D & P-110 \\
\hline Mah, J & P-029 & Mills, $\mathrm{P}$ & P-129 & Parrent, AG & P-079 & Ramsay, D & P-107 \\
\hline Major, P & P-053 & Mirza-Aghazadeh, J & A-07 & Partlo, L & $\mathrm{H}-05$ & Ranawaya, R & P-126 \\
\hline Maloney, William & A-06 & Mistry, N & K-08 & Patel, A & P-119 & Rappaport, J & I-01 \\
\hline Marchand, L & G-04 & Mitchell, JR & $\mathrm{F}-05$ & Patel, AD & P-137 & Rasmussen, $\mathrm{P}$ & F-06 \\
\hline Maroun, F & P-076 & Mitha, A & P-013 & Pelletier, J & P-145 & Reddy, K & $\mathrm{I}-08$ \\
\hline Maroun, F & P-078 & Miyashita, $\mathrm{H}$ & M-03 & Pellis, S & D-06 & Reddy, K & P-140 \\
\hline Martin, C & P-151 & Mohr, G & $\mathrm{I}-01$ & Pelz, David & P-044 & Redekop, G & P-087 \\
\hline Martin, E & P-040 & Mohr, G & $\mathrm{I}-06$ & Peraud, A & P-106 & Redoblado, M & L-03 \\
\hline Martini, J & C-07 & Molina, Carlos & F-02 & Perl, J & F-06 & Reid, R & P-077 \\
\hline Masaryk, T & F-06 & Mondal, S & P-106 & Persad, V & K-04 & Reid, Robert & P-044 \\
\hline Massicotte, E & E-05 & Montanera, W & A-02 & Petrecca K & B-05 & Remillard, G & $\mathrm{H}-02$ \\
\hline Massicotte, E & P-141 & Montanera, W & P-156 & Petruk, K & B-08 & Rempel, J & P-085 \\
\hline Masson, $\mathrm{H}$ & D-02 & Moodley, M & P-031 & Pettersen, JA & P-126 & Rennie, T & P-042 \\
\hline Massoud, F & $\mathrm{J}-07$ & Moore, $\mathrm{M}$ & $\mathrm{J}-08$ & Phan, C & P-153 & Rennie, $\mathrm{T}$ & P-045 \\
\hline Mathieu, J & G-04 & Moosavian, M & A-08 & Phillips, N & $\mathrm{J}-06$ & Rhines, L & P-097 \\
\hline Matsumoto, Yuuri & P-054 & Moran, M & P-117 & Phillips, S & L-02 & Richer, L & C-04 \\
\hline Maxner, CE & $\mathrm{P}-113$ & Morrison, $\mathrm{T}$ & P-065 & Phypers, M & D-01 & Richer, L & P-030 \\
\hline Mayberg, M & F-06 & Motte, J & P-021 & Pickett, GE & $\mathrm{E}-03$ & Rickards, L & P-142 \\
\hline McBeth, PB & B-02 & Moulton, R & M-05 & Pickett, GE & P-079 & Riess, CM & L-01 \\
\hline McBeth, PM & $\mathrm{I}-03$ & Moumdjian, R & P-114 & Pillay, N & $\mathrm{C}-07$ & Roberts, TE & P-112 \\
\hline McCarthy, L & $\mathrm{C}-06$ & Mouradian, Michael & P-152 & Pillay, $\mathrm{N}$ & H-05 & Robillard, A & $\mathrm{J}-07$ \\
\hline McClarty, B & A-03 & Mukherji, Suresh & $\mathrm{P}-123$ & Pirouzmand, F & P-070 & Robinson, C & P-070 \\
\hline McKean, J & P-074 & Muller, P & P-101 & Pirouzmand, F & P-134 & Robinson, C & P-098 \\
\hline McKean, JDS & $\mathrm{P}-058$ & Murdoch, G & $\mathrm{J}-08$ & Pirouzmand, F & P-136 & Roland, E & P-033 \\
\hline McKean, JDS & P-112 & Murphy, W & $\mathrm{C}-07$ & Pirouzmand, Farhad & F-04 & Roland, E & P-035 \\
\hline McKelvey, R & P-093 & Murphy, W & H-05 & Pirouzmand, Farhad & P-103 & Rompre, $\mathrm{P}$ & P-088 \\
\hline McLaughlin, $\mathrm{N}$ & P-015 & Murray, D & P-107 & Pitters, C & P-056 & Rosenblatt, B Pre & s Prize \\
\hline McLaughlin, N & P-016 & Murray, G & P-076 & Porciuncula, J & $\mathrm{J}-02$ & Ross, B & P-020 \\
\hline McLeod, M & P-006 & Murray, G & P-078 & Porter, $\mathrm{P}$ & M-05 & Ross, BC & P-019 \\
\hline McNabb-Baltar, J & G-04 & Murty, N & P-115 & Pourcher, Emmanuelle & P-089 & Ross, $\mathrm{T}$ & F-03 \\
\hline McNeely, D & P-133 & Myles, T & $\mathrm{H}-05$ & Powers, C & D-01 & Rossi, JJ & G-01 \\
\hline Megyesi, J & P-064 & Nagy, A & B-03 & Price, A & M-02 & Roullin, G & P-109 \\
\hline Megyesi, J & P-110 & Naik, S & P-043 & Price, A & P-033 & Rousseau, J & P-007 \\
\hline Megyesi, J & P-111 & Narayanan, S & P-092 & Protti, M & B-08 & Rowed, D & $\mathrm{K}-03$ \\
\hline Megyesi, J & P-118 & Nasreddine, $\mathrm{Z}$ & J-06 & Prout, A & P-042 & Rutka, J & P-106 \\
\hline Megyesi, JF & P-079 & Nguyen, DK & P-059 & Prout, A & P-045 & Rutka, James T & P-054 \\
\hline Mehta, V & M-01 & Nguyen, T & P-124 & Provias, J & P-115 & Sabaz, M & L-03 \\
\hline Mehta, V & P-075 & Nichol, K & P-014 & Puymirat, J & G-01 & Sabbagh, A & P-099 \\
\hline Mehta, V & P-133 & Nikseresht, AR & D-03 & Qian, B & B-01 & Sabha, N & M-07 \\
\hline Mehta, V & P-135 & Nolan, M & L-03 & Qian, B & M-07 & Sabha, N & P-102 \\
\hline Mehta, V & P-074 & Noor, Raza & P-150 & Qian, B & P-080 & Sadanand, V & P-137 \\
\hline Melanson, D & P-099 & Norris, J & P-147 & Qian, BP & B-07 & Sadanand, V & P-138 \\
\hline Mendez, I & I-02 & O’Brien, G & P-076 & Quenneville, S & P-007 & Sadanand, V & P-143 \\
\hline Mendez, I & P-004 & O'Connor, $\mathrm{P}$ & P-094 & Quesney, F & H-06 & Sadanand, Venkat & F-04 \\
\hline Mendez, I & P-006 & Oczkowski, W & P-151 & Quesney, F & $\mathrm{J}-01$ & Sadanand, Venkat & P-103 \\
\hline Mendez, I & P-091 & Oger, J & $\mathrm{J}-02$ & Quesney, F & $\mathrm{J}-05$ & Sade, B & I-06 \\
\hline
\end{tabular}




\begin{tabular}{|c|c|c|c|c|c|c|c|}
\hline Sadi, D & P-006 & Shuaib, Ashfaq & P-152 & Todd, K & M-03 & Wheatley, BM & P-112 \\
\hline Sadi, D & P-090 & Siadati, A & P-097 & Toth, C & P-127 & Wheatley, M & P-074 \\
\hline Sadler, M & P-055 & Siddiqi, ZA & P-050 & Toth, C & P-128 & Whishaw, I & D-06 \\
\hline Sadovnick, AD & $\mathrm{J}-03$ & Siddiqi, ZA & P-131 & Townsend, T & G-03 & White, C & P-127 \\
\hline Sadovnick, AD & P-025 & Sim, V & P-008 & Townsend, T & P-038 & White, C & P-126 \\
\hline Sahlas, DJ & P-147 & Sinclair, B & C-04 & Toyota, B & A-01 & Whitehead, V & J-06 \\
\hline Saint-Cyr Jean A & & Sinclair, B & P-058 & Tremblay, JP & P-007 & Wiley, C & P-064 \\
\hline \multicolumn{2}{|c|}{ Herbert Jasper Prize } & Sinclair, D & P-030 & Tremblay, JP & P-129 & Wilkinson, M & $\mathrm{I}-07$ \\
\hline Salam, Abdul & P-148 & Sinclair, DB & P-043 & Tremblay, J-P & P-130 & Willinsky, R & P-017 \\
\hline Salame, J & P-108 & Singh, Sheila K & B-06 & Tremlett, H & J-02 & Willinsky, R & A-02 \\
\hline Salman, M & C-08 & Singhal, A & M-05 & Triaforos, $\mathrm{R}$ & P-060 & Willinsky, R & P-156 \\
\hline Samangooei, Sh & D-03 & Siu, K & P-105 & Tsai, E & E-01 & Wilson, B & P-101 \\
\hline San Vincente, M & P-063 & Smith, A & P-151 & Tsao, M & A-07 & Wilson, JX & P-064 \\
\hline Sanche, S & P-070 & Smith, T & P-076 & Tu, JV & L-05 & Wine, L & P-051 \\
\hline Santos, AC & P-092 & Snead III, OC & K-08 & Tweed, Douglas & $\mathrm{K}-05$ & Wingerchuk, D & J-04 \\
\hline Santos, C & President's Prize & Snead III, OC & $\mathrm{K}-04$ & Tymianski, M & A- 08 & Wirrell, E & C-07 \\
\hline Saqqur, Maher & F-01 & Snead, Carter O & P-054 & Tymianski, M & $\mathrm{A}-02$ & Wirrell, E & P-020 \\
\hline Saqqur, Maher & $\mathrm{F}-02$ & Snyder, $T$ & P-058 & Tymianski, M & P-156 & Wirrell, E & P-023 \\
\hline Saqqur, Maher & P-148 & Spacey, S & D-05 & Tyson, $\mathrm{R}$ & K-06 & Wirrell, E & P-024 \\
\hline Saqqur, Maher & P-149 & Spears, J & A-08 & Tyson, $\mathrm{R}$ & M-04 & Wong, A & E-05 \\
\hline Sargent, M & P-033 & Spears, J & P-012 & Tyson, $\mathrm{R}$ & P-005 & Wong, Agnes MF & $\mathrm{K}-05$ \\
\hline Sarjeant, R & E-02 & Spivak, C & P-098 & Uchina, Ken & F-02 & Wong, J & P-013 \\
\hline Sasaki, H & P-118 & Spivak, C & P-122 & Van Soelen, J & E-03 & Wong, JH & P-019 \\
\hline Sauvageau, E & P-114 & Squire, Jeremy & B-06 & Vandorpe, Robert & A-06 & Woo, $\mathrm{H}$ & F-06 \\
\hline Schebel, Marcia & P-148 & Steckley, JL & P-073 & Vandorpe, Robert & P-123 & Wood, E & C- 03 \\
\hline Schebel, Marcia & P-149 & Steinbach, M & C-08 & Varma, A & P-101 & Wright, S & M-03 \\
\hline Schroeder, M & B-07 & Steinbok, $\mathrm{P}$ & M-02 & Vennettilli, M & K-03 & $\mathrm{Wu}, \mathrm{A}$ & P-136 \\
\hline Schultke, E & P-105 & Steinke, DE & P-010 & Verma, A & P-084 & Yahya, R & P-139 \\
\hline Schwartz, G & E-06 & Stephan, L & P-130 & Verreault, S & L-07 & Yahya, R & P-154 \\
\hline Schwartz, M & A-07 & Stewart, B & P-153 & Verret, L & $\mathrm{J}-07$ & Yang, V & P-101 \\
\hline Schwartz, M & M-05 & Stewart, J & G-03 & Verret, L & L-07 & Yazdi, J & P-082 \\
\hline Schwartz, M & K-03 & Stewart, J & L-04 & Vezina, JL & $\mathrm{I}-06$ & Yip, S & P-155 \\
\hline Scora, D & A-07 & St-Hilaire, J-M & P-059 & Voll, C & F-03 & Yoo, BG & P-062 \\
\hline Sen, A & P-006 & Stumpf, E & D-02 & Voll, C & P-060 & Yoo, KM & P-062 \\
\hline Seshia, Shashi & $\mathrm{C}-02$ & Stys, P & P-009 & Vorobeychik, G & P-042 & Young, B & P-065 \\
\hline Seshia, Shashi & P-037 & Stys, P & P-124 & Vorobeychik, G & P-045 & Young, B & K-07 \\
\hline Sevick, R & $\mathrm{H}-05$ & Subramaniam, S & P-146 & Vujovic-Zotovic, $\mathrm{N}$ & P-147 & Young, B & P-046 \\
\hline Sevick, R & P-146 & Suchowersky, O & D-06 & Waldkircher- & & Young, B & P-047 \\
\hline Shahrour, K & G-05 & Sutherland, G & K-06 & de Oliveira, RM & P-104 & Young, Bryan & P-044 \\
\hline Shannon, $\mathrm{P}$ & B-03 & Sutherland, G & M-04 & Wallace, MC & A-02 & Yousefipour, Gh & D-03 \\
\hline Shannon, $\mathrm{P}$ & M-07 & Sutherland, G & P-005 & Wallace, MC & P-012 & Zadeh, G & B-01 \\
\hline Shannon, $\mathrm{P}$ & P-102 & Sutherland, G & P-011 & Wallace, MC & P-156 & Zadeh, G & B-07 \\
\hline Shariat, $\mathrm{AH}$ & D-03 & Sutherland, GR & B-02 & Wallace, MC & A-08 & Zadeh, G & M-07 \\
\hline Sharma, M & P-009 & Sutherland, GR & I-03 & Walling, S & I-02 & Zadeh, G & P-080 \\
\hline Sharma, M & P-124 & Svenson, L & P-095 & Walsh, E & P-078 & Zadeh, G & P-102 \\
\hline Sharma, Manju & P-061 & Tabasi, Reza & P-096 & Walsh, KA & P-064 & Zeitouni, A & I-01 \\
\hline Sharma, Nitya nand & $\mathrm{P}-061$ & Tai, $\mathrm{P}$ & P-057 & Walsh, R & P-040 & Zhu, H & F-05 \\
\hline Sharpe, J & C-08 & Tarrant, M & F-05 & Wang J & B-05 & Zifkin, B & $\mathrm{H}-02$ \\
\hline Sharpe, James A & D-08 & Tator, C & E-01 & Wang, Chen xu & P-150 & Zlatescu, M & P-118 \\
\hline Sharpe, James A & P-067 & Taylor, A & $\mathrm{K}-03$ & Warren, KG & P-095 & Zochodne, D & P-128 \\
\hline Sharpe, James & K-05 & Temblay, JP & G-02 & Warren, S & P-095 & & \\
\hline Shemie, $\mathrm{S}$ & C-06 & Ter Brugge, $\mathrm{K}$ & P-154 & Wei, Q & P-100 & & \\
\hline Shoichet, M & E-01 & ter Brugge, $\mathrm{K}$ & P-156 & Wells, J & M-06 & & \\
\hline Shuaib, A & M-03 & ter Brugge, $\mathrm{KG}$ & A-02 & West, M & A-04 & & \\
\hline Shuaib, Ashfaq & F-01 & Terasaki, Mizuhiko & B-06 & West, M & I-05 & & \\
\hline Shuaib, Ashfaq & $\mathrm{F}-02$ & TerBrugge, $\mathrm{K}$ & A-08 & West, M & A-03 & & \\
\hline Shuaib, Ashfaq & P-148 & TerBrugge, $\mathrm{K}$ & P-017 & West, M & P-086 & & \\
\hline Shuaib, Ashfaq & P-149 & Theodorsson, A & P-083 & Westall, C & C-08 & & \\
\hline Shuaib, Ashfaq & P-150 & Thompson, W & P-064 & Wheatley, BM & P-058 & & \\
\hline
\end{tabular}

